\title{
The Exploration of Nanotoxicological Copper and Interspecific Saccharomyces Hybrids
}

\author{
Matthew Joseph Winans PhD \\ West Virginia University, mwinans@mix.wvu.edu
}

Follow this and additional works at: https://researchrepository.wvu.edu/etd

Part of the Biochemistry Commons, Cell Biology Commons, Food Biotechnology Commons, Food Microbiology Commons, Genetics Commons, Genomics Commons, Integrative Biology Commons, Laboratory and Basic Science Research Commons, Molecular Biology Commons, Molecular Genetics Commons, Other Microbiology Commons, Other Pharmacology, Toxicology and Environmental Health Commons, Structural Biology Commons, Systems Biology Commons, and the Toxicology Commons

\section{Recommended Citation}

Winans, Matthew Joseph PhD, "The Exploration of Nanotoxicological Copper and Interspecific Saccharomyces Hybrids" (2020). Graduate Theses, Dissertations, and Problem Reports. 7648. https://researchrepository.wvu.edu/etd/7648

This Dissertation is protected by copyright and/or related rights. It has been brought to you by the The Research Repository @ WVU with permission from the rights-holder(s). You are free to use this Dissertation in any way that is permitted by the copyright and related rights legislation that applies to your use. For other uses you must obtain permission from the rights-holder(s) directly, unless additional rights are indicated by a Creative Commons license in the record and/ or on the work itself. This Dissertation has been accepted for inclusion in WVU Graduate Theses, Dissertations, and Problem Reports collection by an authorized administrator of The Research Repository @ WVU. For more information, please contact researchrepository@mail.wvu.edu. 
The Exploration of Nanotoxicological Copper and Interspecific Saccharomyces Hybrids

\section{Matthew Joseph Winans}

Dissertation submitted to the Eberly College of Arts and Sciences

at West Virginia University

In partial fulfillment of the requirements for the degree of

Philosophiae Doctor (Ph.D.)

in

Biology

Jennifer E. G. Gallagher, Ph.D., Chairperson

Jennifer S. Hawkins, Ph.D.

Jonathan R. Cumming, Ph.D.

Daniel G. Panaccione, Ph.D.

Kang Mo Ku, Ph.D.

Department of Biology

Morgantown, West Virginia

2020

Keywords: nanotechnology, saccharomyces, nanoparticle, copper, cellulose, genetic, fermentation, membrane, zinc, lipid

(C) 2020 by Matthew J. Winans. All rights reserved. 


\section{ABSTRACT \\ The Exploration of Nanotoxicological Copper and Interspecific Saccharomyces Hybrids}

\section{Matthew J. Winans}

$\mathrm{N}$ anotechnology takes advantage of cellular biology's natural nanoscale operations by interacting with biomolecules differently than soluble or bulk materials, often altering normal cellular processes such as metabolism or growth. To gain a better understanding of how copper nanoparticles hybridized on cellulose fibers called carboxymethyl cellulose (CMC) affected growth of Saccharomyces cerevisiae, the mechanisms of toxicity were explored. Multiple methodologies covering genetics, proteomics, metallomics, and metabolomics were used during this investigation. The work that lead to this dissertation discovered that these cellulosic copper nanoparticles had a unique toxicity compared to copper. Further investigation suggested a possible ionic or molecular mimicry scenario with zinc, likely involving the Zrt family of zinc transporters and involving arrestin mediated endocytosis. Reactive oxygen species were generated by copper nanoparticles that induced lipid peroxidation, altering the phosphatidylcholine and phosphatidylethanolamine membrane composition, resulting in a disfigured cell surface. Following this study, I designed experiments aimed at furthering this dissertation's focus on metabolism by describing the metabolism of a novel species, Saccharomyces arboricola, thus filling a gap in knowledge in the industrial fermentation field. Low levels of endogenous amino/organic acids separated $S$. arboricola from the $S$. cerevisiae industrial strains and their interspecific hybrids showed a mosaic metabolic profile of parental strains. Overall, my dissertation research identified mechanisms of cellulosic copper nanotoxicity that included transport, metal homeostasis, reactive oxygen species, and the cellular membrane composition. Perturbations of Saccharomyces yeast by exogenous exposure to nanocopper or by interspecific hybridization had effects on the cellular metabolism. 


\section{PREFACE}

Characterization of nanoparticle toxicity, transport, and the impact on metabolism of cellulosic copper nanoparticles was the objective of this dissertation. Understanding of the ways in which nanomaterials differ from traditional biological toxins is necessary for responsible technological development and sustainability. However, the exact method in which copper nanoparticles induce toxicity has not been determined, representing a major gap in our understanding. Defining how novel nanomaterials induce toxicity is exceedingly important as the ubiquity of nanotechnology is steadily increasing with time. The use of nanomaterials in intimate items such as sunscreens, cosmetics, anti-microbials, and medical devices warrants exploration and understanding.

My central hypothesis was that the positively charged cellulose copper nanoparticles are attracted to the negative charge of the cell wall where importation leads to ROS damage of the cellular membrane lipids and biomolecules initiating toxicosis of the yeast cell. The scientific premise of this hypothesis was formed from observations that suggest a major portion of the nanoparticles are not found within the cell, a general tendency for endocytosis of nanomaterials, and preliminary research suggesting that halted endocytosis rescues $\mathrm{CMC}-\mathrm{Cu}$ nanotoxicity. This dissertation is innovative because we are using multiple disciplines including physics, chemistry, and systems biology to explore the frontier of nanotechnology involving novel $\mathrm{CMC}-\mathrm{Cu}$ nanocomposites. $\mathrm{My}$ rationale is that by using utilizing multiple approaches, we can create a profile of the toxicological damage which gives insight into the drivers of $\mathrm{CMC}-\mathrm{Cu}$ nanotoxicity. The objective of this dissertation was met by experimentation of the following two specific aims.

Aim 1: Characterize the Unique Nanotoxicity of CMC-Cu. By using multiple viability assay, genetic screens, and metabolomics assessments I determined the genetic, metabolic, and protein components important for resolving the nanotoxicological mechanisms.

Aim 2: Determine Cellular Localization of Nanoparticles. By using advanced microscopy and genetic approaches we determined the interaction and localization important for resolving the nanotoxicological mechanisms.

In the second and third chapters, $\mathrm{CMC}-\mathrm{Cu}$ nanotoxicity was investigated using genetically diverse Saccharomyces cerevisiae strains. The growth inhibition, metal homeostasis, genetic regulation, protein content, morphology, and metabolism was explored. Many lines of evidence found in this dissertation aligned with literature and suggested that $\mathrm{CMC}-\mathrm{Cu}$ 's affects lipid homeostasis, resulting in a flux in lipid metabolism via the Kennedy pathway that synthesizes membrane lipids. The fourth chapter of this dissertation shifts focus from the Kennedy pathway to the fermentation pathway of Saccharomyces interspecific hybrids. Investigation into the metabolome is upmost interest because this technique captures the cell's rapid and entire response to perturbation, in contrast to the gradual measurements of the proteomics or genomics. Lipid metabolism was measured in response to the exogenous CMC-Cu perturbation in Chapters Two and Three, while a more in-depth focus on metabolism was taken in Chapter Four by endogenous changes to the Saccharomyces yeast cellular composition by interspecific hybridization. 


\section{ACKNOWLEDGMENTS}

Most importantly, I must acknowledge my doctoral advisor, Dr. Jennifer Gallagher. I first met Jen in 2015 during a visit I made to West Virginia University's Life Science Building, I was working as a traveling salesman for my family's janitorial company at the time. Her inquisitiveness, excitability, and dedication to science was impressive to say the least. As my academic mentor, Jen encouraged me to pursue new and exciting avenues in science. She respected my career goals, extracurricular undertakings, and work-life balance. She truly invested her time to help me grow as her graduate student and as a person, for which I am extremely grateful. I was fortunate to have found an advisor that provided constructive criticism, gave support when needed, and had confidence in my abilities to conquer my goals.

My graduate committee members - Dr. Jonathan Cumming, Dr. Kang-Mo Ku, Dr. Daniel Panaccione, and Dr. Jennifer Hawkins were particularly instrumental throughout my graduate studies. Their time and valuable feedback challenged me to think critically and taught me the joy in performing literature research. I am especially thankful for the side-by-side work experience I gained with Dr. Cumming and Dr. Ku during my nanoparticle research. I also need to thank Dr. Rita Rio who gave me courage early in my graduate education by accepting me to rotate in her lab during my first semester.

A special thank you is in order to my sensei, Dr. Hiroshi Kitagaki (北垣浩志) of Saga University (佐賀大学), Japan, who supported and tolerated me during my time as a JSPS-NSF fellow. Additionally, Dr. Kimberly Quedado, Dr. Alan Bristow, and Dr. Gloria Oporto were extremely helpful during the NSF-IGERT fellowship.

During my first visit, I met my soon to be lab mates, Apoorva Ravishankar and Michael Ayers, who became some of my best friends through my graduate education. We shared laughter, frustrations, and worked relentlessly together. Phil Chapman, Tyler Sizemore, Justin Mathias, Adam Pollio, Miguel Medina Munoz, and the fellow Biology Graduate Student Association members were especially helpful and supportive. There are too many graduate students to name all that helped me within and outside of the Life Sciences Building, but thank you.

Life as a researcher in Japan was amazing and I am thankful for the people with whom I have rich memories. Thank you Miyuki Miyagawa (宮川幸), Yasuhiro Takaki (高木康裕), Mikako Hirano (平野美佳子) and Alan Kyo (許志豪) for all the great experiences. Thank you Yuki Fujimaru (藤 丸裕貴), and Yuki Yamamoto (山本裕貴) for all the help in my new lab.

Lastly, I couldn't have gone as far in my education without the encouragement from my family. Words cannot describe the amount of support and confidence my parents, Joseph Matthew Winans and Martha Jean (Brletrick) Winans gave me throughout my education. As a child my mother went above and beyond to help me learn and my father provided corrections to life's trial and errors. I am eternally grateful for your teamwork, love, and dedication throughout the years. Furthermore, my extended family deserves thanks as their contributions were instrumental in my success, livelihood, and education. 


\section{TABLE OF CONTENTS}

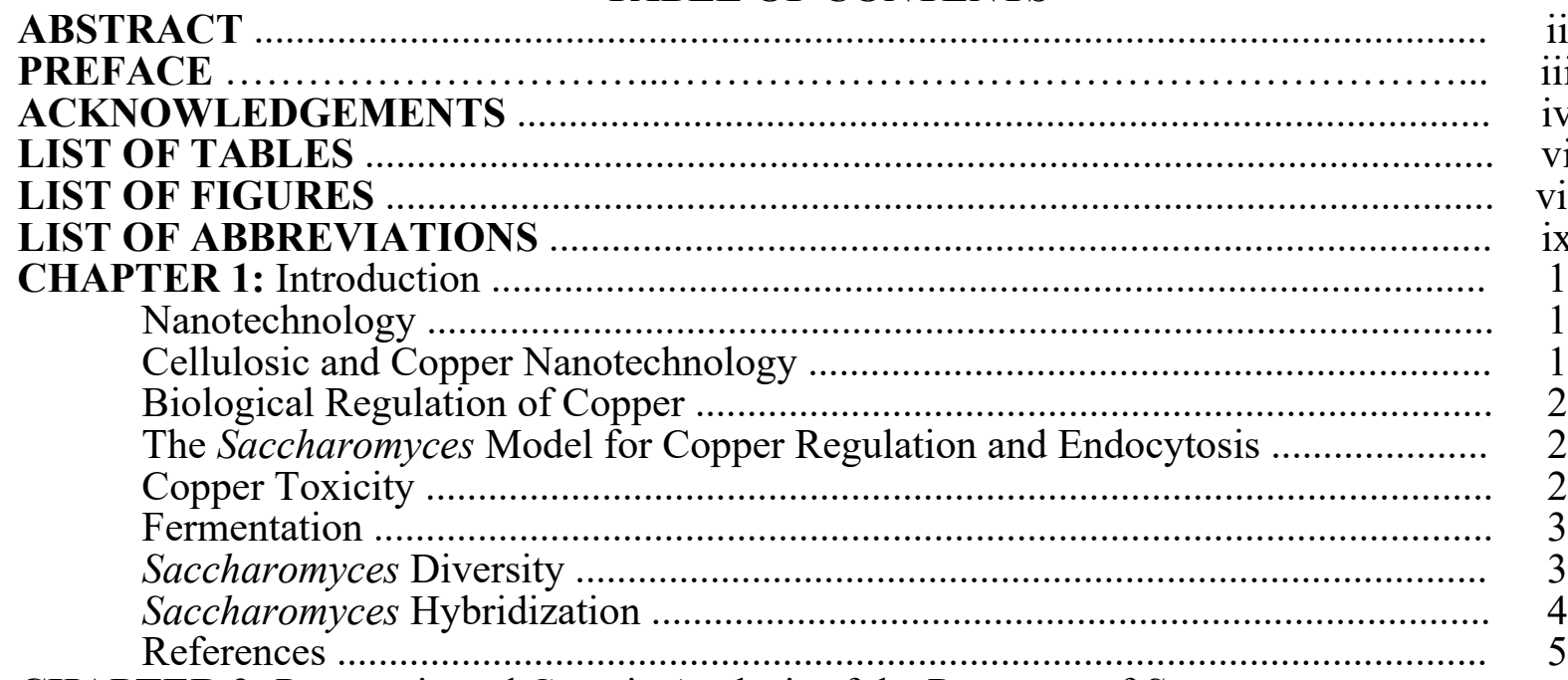

CHAPTER 2: Proteomic and Genetic Analysis of the Response of S. cerevisiae to

Soluble Copper Leads to Improvement of the Antimicrobial Function of Cellulosic Copper Nanoparticles ................................................................

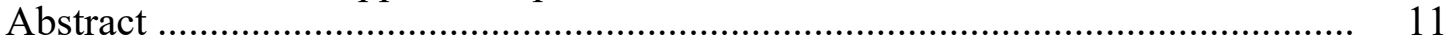

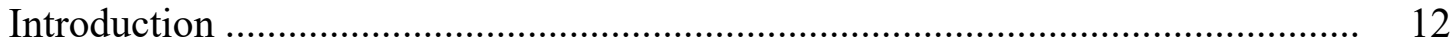

Materials and Methods ……………………………………………………. 12

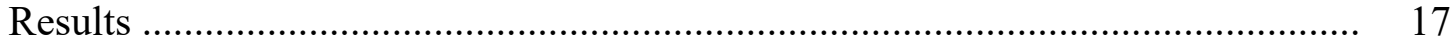

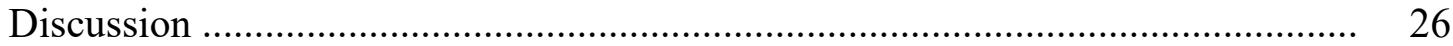

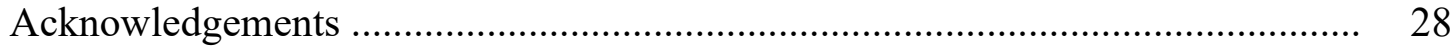

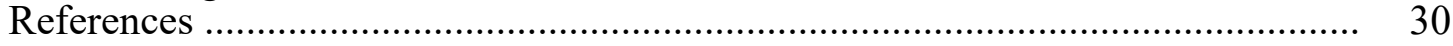

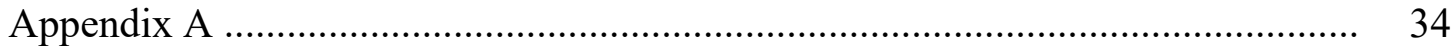

CHAPTER 3: Metallomic and Lipidomic Analysis of S. cerevisiae Response to

Abstract

Cellulosic Copper Nanoparticles Uncover Drivers of Toxicity ................... $\quad 42$

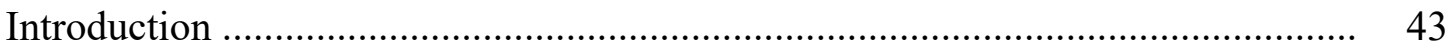

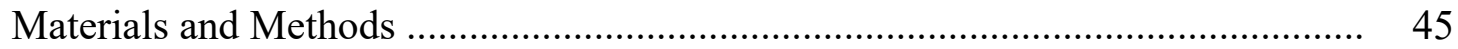

Results and Discussion ............................................................................ 48

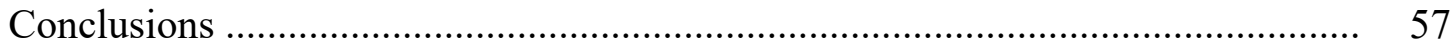

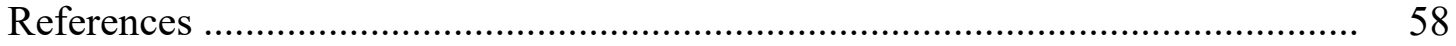

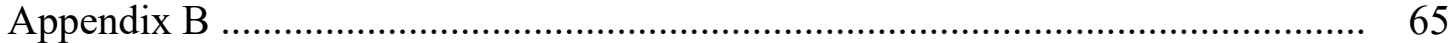

CHAPTER 4: Saccharomyces arboricola and its Hybrids' Propensity for Sake

Production: Interspecific Hybrids Reveal Increased Fermentation

Abilities and a Mosaic Metabolic Profile ............................... 71

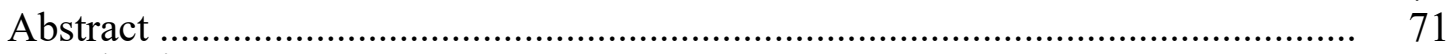

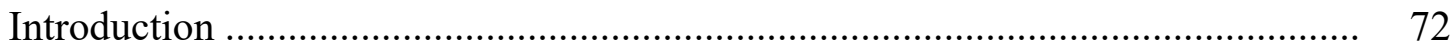

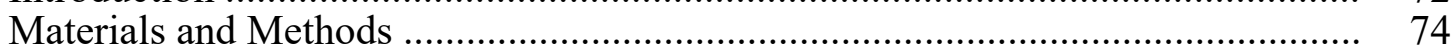

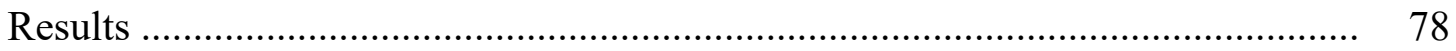

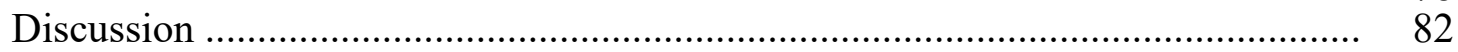

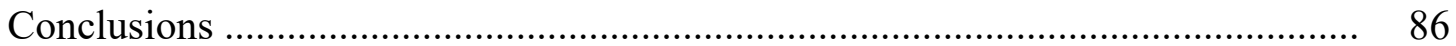

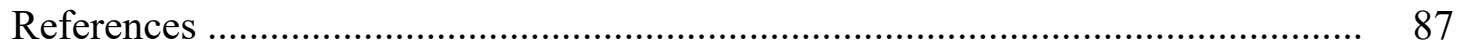

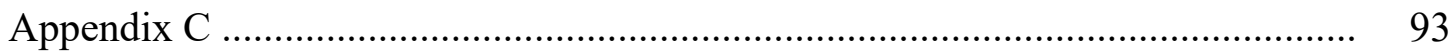

CHAPTER 5: General Conclusions ........................................................................... 100

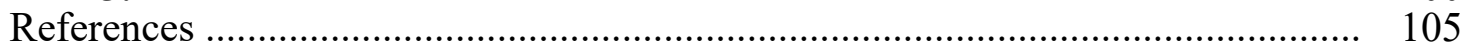




\section{LIST OF TABLES}

2-1. Examples of Proteins Differentially Expressed (Log 2-Fold Ratio) Between S288c

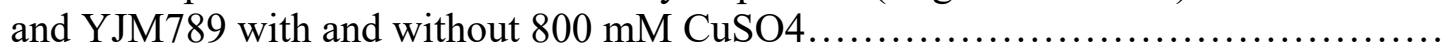

2-S1. Relative Protein Levels of S288c (S96) and YJM789 Cells Grown in YM with

Lysine or YM with Excess $\mathrm{CuSO}_{4}$, Shown as Log2....

2-S2. Common Contaminant List for Proteomics that were Screened During Searches....

2-S3. Growth Values of S288c (S96) and YJM789 Haploid Recombinant Segregants at 12 and 18 -Hours with $50 \mu \mathrm{M} \mathrm{CuSO}$

2-S4. SNP LOD Mean for 12-Hour Exposure.

2-S5. SNP LOD Mean 18-Hour End Point Exposure.

3-1. Connecting Letters Statistical Report for Copper and Zinc Levels by Strain and Treatment... 50

3-S1. Normalized Gas Chromatography Electrospray Ionization Mass Spectrometry Data........ 68

3-S2. Loading Matrix for PLS-DA Lipidomic Assay............................................... 68

4-1. Yeast Strains Used During Hybridization Study .......................... 74

4-S1. Endogenous Metabolite Loading Matrix.............................. 96

4-S2. Connecting Letters Report of Significance for Sake Fermentations............. 97

4-S3. Sake Metabolite Loading Matrix...................................... 98 


\section{LIST OF FIGURES}

2-1. Impact of Genetic Variation in S. cerevisiae to Soluble Copper and Cellulosic Hybrid Copper Nanoparticles

2-2. Rescue of Copper Nanoparticle Sensitivity with Antioxidants ...

2-3. Internal Levels of Copper in Yeast Treated with Different Sources of Copper and Antioxidants.

2-4. Physical Interaction of Copper Nanoparticles and Yeast Cells.

2-5. Acute Exposure of Yeast to Copper Nanoparticles.

2-6. Impact of Zinc Transporters and Zinc on Copper Response

2-7. Addition of Zinc to Copper Nanoparticles Altered Growth Inhibition.

2-S1. Flow Cytometry of Individually Stained Yeast.

2-S2. Hierarchical Clustering of Protein Levels from Proteomic Analysis of Yeast

Treated with Copper Reveals Inherent Characteristics of Yeast Strains.

2-S3. Growth Assays of Genetically Diverse Yeast in Media with Excess and Limited Copper.

2-S4. Genomic Loci Linked to Copper Response

2-S5. Serial Dilutions of Yeast Growth in the Presence of Elevated Zinc

2-S6. Protein Alignment of Zrt2 from S288c and YJM789

3-1. Copper and Zinc Metal Homeostasis Measured by Inductively Coupled Plasma Optical Emissions Spectroscopy During CMC-Cu Toxicity

3-2. Cellular Toxicity Profiles Including ROS Generation and Biomolecule Damage...

3-3. Lipid Metabolic Profiling in Response to $\mathrm{CMC}-\mathrm{Cu}$ Treatment.

3-4. Scanning Electron Micrographs of Yeast's Cellular Surface.

3-5. Model of CMC-Cu Toxicity 56

3-S1. Copper Homeostasis During CMC-Cu Toxicity Represented in Treatment Groups. 65

3-S2. Lipidomic Statistics for CMC-Cu Treatment.

66 
3-S3. Whole Lipidome Heatmap for CMC-Cu Treatment.......................... 67

3-S4. Variable Importance Projection Score Plot................................. 68

4-1. Yeasts Endogenous Metabolic Profiles................................... 79

4-2. Cryotolerance of Parental and Hybrid Yeasts................................. 79

4-3. Phenotypic Characteristics of Hybrids and Parental Yeast...................... 80

4-4. Sake Fermentation Profiles of $S$. arboricola Interspecific Hybrids and Parental

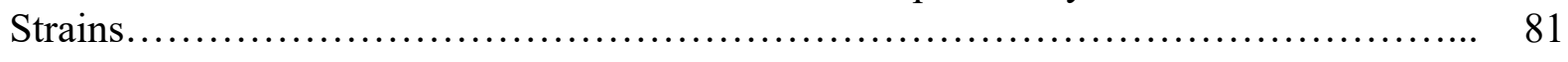

4-S1. 3D PCA Score Plot of the Saccharomyces Yeast Endogenous Metabolites........ 93

4-S2. Spore Staining and PCR DNA Confirmation............................ 94

4-S3. Sake Fermentation Metabolic Profile of S. arboricola Interspecific Hybrids and Parental Strains Sample by Sample......................................... 95 


\section{LIST OF ABBREVIATIONS}

2,7-dichlorofluorescin diacetate

DCFDA

4-(2-hydroxyethyl)-1-piperazineethanesulfonic acid

HEPES

Accidental cell death

ACD

Adenosine triphosphate

ATP

Analysis of variance

ANOVA

Arrestin-related trafficking adaptors

ART

Ascorbic acid

$\mathbf{A A}$

Atomic absorption spectroscopy

AAS

Silver

Silver nanoparticles

Ag

Basepairs

$\operatorname{AgNP}$

Bathocuproinedisulfonic acid

BP

Carboxymethyl cellulose

Carboxymethyl cellulose copper nanoparticles

Cellulosic cuprous nanoparticles

Chromosome

BCS

Copper

Copper nanoparticle

Cupric

CMC

$\mathrm{CMC}-\mathrm{Cu}$

c-CuNPs

Chr

$\mathrm{Cu}$

CuNP

Cuprous

$\mathrm{Cu}^{+2}$

Copy number variation

$\mathrm{Cu}^{+}$

Cytochrome c oxidase

Deoxyribonucleic acid

Dimethyl sulfoxide

CNV

$\mathrm{CcO}$

DNA

DMSO

Dimethyl trisulfur

DMTS

Endoplasmic reticulum

ER

Fluorescein isothiocyanate

FITC

Fluorescence

FC

Food and Drug Administration

FDA

Gas chromatography-electron spray ionization mass-spectroscopy

GC-ESI-MS

Gas chromatography-flame ionization detection

Generally Recognized as Safe

Glutathione

GC-FID

GRAS

GSH

Glutathione oxidized

GSSH

Head space-solid phase microextraction

High collisional cell

HS-SPME

Histidine, uracil, leucine, and methionine

HCD

Human Metabolome Database

HULM

HMDB

Inductively coupled plasma - optical emission spectroscopy

ICP-OES

Inner membrane space

Institute for Research on Cancer and Aging

Iron

IMS

IRCAN

Linear trap quadrupole

Fe

Logarithm of odds

LTQ

LOD 
Madison Metabolomics Consortium Database

Malondialdehyde

MMCD

Medium chain fatty acids

MDA

MilliQ

MCFA

Multi-walled carbon nano tubes

MQ

$\mathrm{N}$-acetylcysteine

MWCNT

N-methyl-N-TMS-trifluoroacetamide

NAC

Nanoparticle

MSTFA

National Institute of Standards and Technology

NP

National Research Institute of Brewing

NIST

Nicotinamide

NRIB

Optical density units

NAM

Partial least squared discriminant analysis

ODu

Peptide spectrum matches

PLS-DA

Phosphate buffered saline

PSM

Phosphatidylcholine

PBS

Phosphatidylethanolamine

PC

Polymerase chain reaction

PE

polyvinyl alcohol

PCR

Principal component

Principal component analysis

PVA

PC

Pulse-field gel electrophoresis

PCA

Quantitative trait loci

Reactive oxygen species

PFGE

Reduction-oxidation

QTL

ROS

Regulated cell death

Redox

RCD

Rhodamine B hexyl ester

S. arboricola 350 and S. cerevisiae M22

RhodB

Scanning electron microscopy

SAM

Soft independent modeling by class analogy

Species

Species (multiple)

Standard error

Synonym

Tandem mass tags

SEM

SIMCA

Sp

Spp

SE

Syn

TMT

Thiobartic Acid

TBA

Thiobartic Acid Reactive Substances

TBARS

Triacylglycerols

TG

Tricarboxylic acid cycle

Tukey-Kramer honest significance difference

TCA

Yeast Metabolome Database

Tukey-HSD

YMDB

Yeast minimal media

YM

Zn

Zrt- and -Irt like protein

ZIP 


\section{CHAPTER 1}

\section{Introduction}

\section{Nanotechnology}

The capacity to manipulate and create molecules on the smallest of scales is nanotechnology. Revolutionary change in industry, science, and medicine has emerged as nanotechnology is introduced into products to improve their performance or alter their properties. Nanosized (syn. ultrafine) particles have a dimension less than $100 \mathrm{~nm}$ in size. The physical, chemical, and functional properties of particles on the nanometer scale often differ from their dissolved or larger particle counterparts of the same elemental species ${ }^{1}$. Nanotechnology has experienced a surge in research efforts during the last decade due to the prevalence of nanotechnology in consumer products as well as progress in the field. Currently, there is a worldwide push from the United States, Japan, Germany, and other government organizations to understand the transition of nanotechnology from the lab bench to the commercial marketplace ${ }^{2}$. Several nano-innovations gained through research include the improvement of batteries via lithium iron phosphate nanoparticles ${ }^{3}$, production of incredibly waterproof clothing via construction of silicone nanofilaments on polyester fibers ${ }^{4}$, and silver antimicrobial nanoparticles embedded in clothing to destroy odor-causing bacteria ${ }^{5}$. Nanoparticles (NPs) are ruled, in part, by two size-dependent effects: surface atom availability which is scalable, and the discontinuous quantum effect owing to the completion of delocalized electron shells ${ }^{1,6,7}$. Cellulose is used as an organic support structure on which copper nanoparticles can be attached. Previous reports focused on copper nanoparticles functionalized on cellulose have shown these materials to be antimicrobial ${ }^{8-14}$. The mechanism by which these unique hybrid materials induce toxicity have yet to be well understood. Chapters Two and Three address the nanotoxicological gap in knowledge of cellulosic copper nanoparticles.

\section{Cellulosic and Copper Nanotechnology}

Cellulose is an abundant renewable resource. It is a water-insoluble fibrous substance composed of high molecular weight homopolymers of $\beta$-1,4-linked anhydro-D-glucose units ${ }^{15}$. Utilization of cellulosic raw material protects copper nanoparticles from self-aggregation and fast oxidation $^{13,16,17}$, a common problem with metallic nanoparticles ${ }^{18}$. The high number of Nacarboxyl groups facilitates copper's reduction on the cellulose derivative carboxymethyl cellulose (CMC) and makes it an appealing organic support structure on which to construct copper nanoparticles ${ }^{13,19}$. These copper nanoparticles are synthesized in situ on these CMC microfibril strands and exhibit mostly $\mathrm{Cu}^{+}$and $\mathrm{Cu}^{0}$ forms averaging $15 \mathrm{~nm}$ in diameter ${ }^{13}$. There are several technique variations in the synthesis of copper nanoparticles including thermal reduction, chemical reduction, sono-chemical reduction (use of ultrasound to alter chemical activity in liquids), metal vapor synthesis, vacuum vapor deposition, radiation methods, microemulsion techniques, and laser ablation. Variations in the copper composition and cellulose biopolymer have been successfully synthesized resulting in changes to their interaction with microorganisms ${ }^{9,10,12,20}$. Furthermore, the hybrid cellulosic-copper nanoparticle materials have been effectively incorporated into both a thermoplastic resin and polyvinyl alcohol with the intent to increase the utility of low-value wood products. The feasibility to use these antimicrobial films in the packaging and healthcare industry 
needs to be assessed through systematic analysis. Specifically, assessments of the toxicity and cellular response to c-CuNP materials are needed.

\section{Biological Regulation of Copper}

Copper nanoparticles are a promising technology that can inhibit pervasive drug-resistant pathogens, nosocomial pathogens, and food spoilage microorganisms that pose a serious threat to human and livestock health ${ }^{21}$. Copper is essential to organisms at trace levels; at higher concentrations, it becomes toxic. Human cells have been shown to have a low sensitivity to copper, a transition metal ${ }^{22}$, while microorganisms maintain a high sensitivity ${ }^{23}$. The response induced by copper in microorganisms has been widely studied. The expression of genes in response to copper is tightly regulated to maintain a fine balance of this essential, yet damaging metal in all organisms ${ }^{24-26}$. Copper's regulation also includes compartmentalization of copper enzymes, the use of metallochaperones to deliver copper to targets, and the localization and turnover of copper transporters ${ }^{27-30}$. Copper is used sparingly for protein functions, usually as an electron donor/acceptor, in which it switches redox states between $\mathrm{Cu}^{+}$and $\mathrm{Cu}^{+2}$ or as an electron carrier ${ }^{31}$.

\section{The Saccharomyces Model for Copper Regulation and Endocytosis}

The eukaryotic organism Saccharomyces cerevisiae specifically imports vital copper to the Golgi apparatus for incorporation into cuproenzymes and distribution in the mitochondria ${ }^{32,33}$. Two metal cell surface reductases, Fre1 and Fre2, facilitate copper reduction from $\mathrm{Cu}^{+2}$ to usable $\mathrm{Cu}^{+34,35}$. In copper-deficient environments, Mac1 induces CTR 1 transcription, the gene that encodes the integral plasma membrane protein and high-affinity copper transporter which mediates cuprous $\left(\mathrm{Cu}^{+}\right)$uptake ${ }^{36-39}$. This protein has been shown to be internalized for vacuole degradation through Rsp5 dependent endocytosis regulated by copper concentrations above $50 \mu \mathrm{M}^{40}$. Once inside the vacuole, the $\mathrm{Ctr} 1 / \mathrm{Cu}$ complex is degraded ${ }^{40}$. Copper can then be chaperoned to areas of the cell as needed for a multiplicity of functions including mitochondrial oxidative phosphorylation and protection against oxidative stress ${ }^{40}$. In high concentrations of copper, the transcription factor Cup2 induces transcription of CUP1 and SOD1 encoding metallothiones ${ }^{41-43}$. Mac1 and Cup2 work together by targeting opposing functions in maintaining a proper copper balance.

Yeast contain a family of arrestins implicated to be a general mechanism for regulating transporters via endocytosis ${ }^{44}$. Endocytosis internalizes cargo from the plasma membrane and has a critical role in nutrient uptake, damaged protein turnover, membrane composition, and the response to extracellular signals. In Saccharomyces cerevisiae, 14 arrestin-related trafficking adaptors (ART) have been identified through sequence analysis. Yeast $\alpha$-arrestins function to target specific plasma membrane proteins for the endocytic system ${ }^{45}$. Ubiquitination by Rsp5, an E3 ubiquitin ligase, of the cargo and endocytic adaptors which link membranes, cargo, and endocytic machinery occur by recruitment from PY (PPXY) motifs on the arrestins ${ }^{46-48}$. Arrestins recruit endocytic promotion factors such as adaptins and clathrin ${ }^{49}$. The mechanisms for understanding cargo selection is not well understood, but evidence from multiple publications suggests the arrestins function to be a multipurpose mediator to the homeostasis of proteins in the plasma membrane ${ }^{50,51}$.

\section{Copper Toxicity}

How antimicrobial metals disrupt cell growth is determined by the physical and chemical properties of the metal and associated biomolecules. Important determinants of metal antimicrobial toxicity are donor atom selectivity, speciation, and reduction potential ${ }^{52,53}$. Excessive copper 
induces cellular damage by generating reactive oxygen species (ROS) (eq.1) $\mathrm{Cu}^{+}+\mathrm{H}_{2} \mathrm{O}_{2} \rightarrow$ $\mathrm{Cu}^{+2}+\mathrm{OH}^{-}+\mathrm{OH}^{* 54,55}$ and by depleting sulfhydryls, including cysteines and glutathione. Glutathione is a potent antioxidant that may be oxidized to form a disulfide bond between two cysteines molecules (RSSR). This cyclic reaction is represented in equations 2 and 3 (eq.2)

$2 \mathrm{Cu}^{+2}+2 \mathrm{RSH} \rightarrow 2 \mathrm{Cu}^{+}+\mathrm{RSSR}+2 \mathrm{H}^{+} \quad$ (eq.3) $\quad 2 \mathrm{Cu}^{+}+2 \mathrm{H}^{+}+\mathrm{O}_{2} \rightarrow 2 \mathrm{Cu}^{+2}+\mathrm{H}_{2} \mathrm{O}_{2}{ }^{56}$. Transition metals not under control by the cell cause damage to membranes, proteins, and DNA ${ }^{57}$. The reactivity of metals follows the hard-soft acid base theory which orders transition metals based on their preference for a ligand ${ }^{58,59}$. For example, cysteine thiolates of metallothioneins form tight associations with monovalent copper ${ }^{60}$. The metal's antimicrobial toxicity is generally proportional to their affinity to sulfur ${ }^{61-63}$. The depletion of antioxidants that combat ROS facilitates damage to biomolecules.

\section{Fermentation}

Eukaryotic yeast have the ability to perform catabolism via oxidative respiration, which generates endogenous ROS in the presence of oxygen, or via fermentation. Their pathway preference depends on their environmental conditions. In high concentrations of sugars, the Crabtree effect represses oxidative respiration for fermentation ${ }^{64,65}$. Crabtree positive yeast emerged approximately 100 million years ago, 25 million years after the appearance of modern fruiting plants ${ }^{66}$. Cellular energy during fermentation primarily comes from the glycolysis pathway, but a diauxic shift occurs when six-carbon sugars are depleted. Ethanol molecules are oxidized into $\mathrm{CO}_{2}{ }^{64,67}$. Fermenting yeast accumulate ethanol, which inhibits the growth of many microorganisms. Once the sugars are depleted, the ethanol is then catabolizes for energy. While the Crabtree effect appeared after the divergence of $S$. cerevisiae from the Kluyveromyces lactis linage ${ }^{66}$, the diauxic shift appeared in two parts ${ }^{68}$, first with the gene duplication of $A D H 1$ to $A D H 2{ }^{69}$ then from the global metabolic rewiring of respiratory regulation ${ }^{70}$. Yeast strains used in the beverage fermentation industry are the ideal candidate for an investigation into metabolism because of their niche domestication dependent on environmental nutrients, their well-characterized pathways, and societal relevance.

\section{Saccharomyces Diversity}

The Saccharomyces yeast is likely the oldest domesticated organism, albeit not intentionally. Its use in baking bread and making alcoholic beverages started as an inadvertent success by some ancient cultures, a practice still used today. Of the budding yeast clade known as Saccharomyces, there are eight species: $S$. cerevisiae, $S$. uvarum, $S$. mikatae, S. kudriavzevii, $S$. arboricola, $S$. eubayanus, $S$. jurei $i^{71-79}$. The divergence between $S$. cerevisiae strains is $0.1-0.6 \%$, but between $S$. cerevisiae and other Saccharomyces members, the divergence is $15-30 \% 0^{80,81}$. This Saccharomyces nucleotide divergence is similar to the human to bird comparison, but with the advantage of a physiological and structural similarity of human to chimp ${ }^{82}$. Chapter Four focused on an industrially novel yeast strain named $S$. arboricola, which was first isolated in 2008 from the tree bark of the Fagaceea family in China ${ }^{72}$ before isolation in Taiwan ${ }^{75}$ and New Zealand ${ }^{83}$. $S$. arboricola has a high quality annotated genomic sequence that when compared to $S$. cerevisiae shows a reciprocal translocation on chromosome (chr.) IV and XIII, inversion on chr.VI, high presence of non-syntenic genes near telomeric regions, lack of introgression, and 4 novel genes ${ }^{84}$. The metabolism of $S$. arboricola lacked a detailed characterization and represented a gap in Saccharomyces metabolism. 


\section{Saccharomyces Hybridizations}

Yeast strain development today is similar to agricultural and livestock techniques that include hybridization, mutagenesis, selection, screening, genetic engineering, synthetic biology, and harvesting non-saccharomyces yeast for regional diversity. Currently, there is an on-going wave of secondary domestication at the laboratory scale from academic, government, and industry laboratories. Selectively breeding yeast traditionally creates intraspecific progeny, between two yeast strains, for improvement of fermentation robustness and sensory characteristics. Interspecific hybrids, between two yeast species, have been very useful in society and have often produced phenotypes that surpass their parents. For example, S. pastorianus and S. bayanus are two natural hybrids identified in the Saccharomyces group ${ }^{85-87}$ that are important to the brewing industry for their metabolic fermentation profiles. In Chapter Four, I characterized the endogenous and exogenous metabolism of $S$. arboricola and newly generated $S$. cerevisiae x $S$. arboricola interspecific hybrids to profile the metabolic differences between the species and to explore their propensity for industrial fermentation. 


\section{References}

1. Roduner, E. Size matters: Why nanomaterials are different. Chem. Soc. Rev. 35, 583-592 (2006).

2. Vance, M. E. et al. Nanotechnology in the real world: Redeveloping the nanomaterial consumer products inventory. Beilstein J. Nanotechnol. 6, 1769-1780 (2015).

3. Xiang, K. et al. Accommodating High Transformation Strains in Battery Electrodes via the Formation of Nanoscale Intermediate Phases: Operando Investigation of Olivine NaFePO 4. Nano Lett. 17, 1696-1702 (2017).

4. Zimmermann, J., Reifler, F. A., Fortunato, G., Gerhardt, L. C. \& Seeger, S. A simple, onestep approach to durable and robust superhydrophobic textiles. Adv. Funct. Mater. 18, 3662-3669 (2008).

5. W. Lem, K. et al. Use of Nanosilver in Consumer Products. Recent Pat. Nanotechnol. 6, 60-72 (2011).

6. Roduner, E. Nanoscopic Materials : Size-Dependent Phenomena and Growth Principles. (Cambridge: Royal Society of Chemistry, 2014).

7. Jortner, J. Cluster size effects. Zeitschrift für Phys. D Atoms, Mol. Clust. 24, 247-275 (1992).

8. Rong-Mullins, X. et al. Proteomic and genetic analysis of the response of S. cerevisiae to soluble copper leads to improvement of the antimicrobial function of cellulosic copper nanoparticles. Metallomics 9, 1304-1315 (2017).

9. Winans, M. J., Gallagher, J. E. G., Jaczynski, J. \& Oporto, G. Pick Your Poison: Benzalkonium Chloride and Copper Enable Nanocellulose Derivatives to Form Antimicrobial Properties Against a Spectrum of Microorganisms. bioRxiv 783076 (2019). doi:10.1101/783076

10. Zhong, T., Oporto, G. S., Jaczynski, J. \& Jiang, C. Nanofibrillated Cellulose and Copper Nanoparticles Embedded in Polyvinyl Alcohol Films for Antimicrobial Applications. Biomed Res. Int. 2015, 1-8 (2015).

11. Jiang, C., Oporto, G. \& Zhong, T. Effect of TEMPO Nanofibrillated Cellulose Content on Copper Release from Antimicrobial Polyvinyl Alcohol Films. (2015).

12. Jiang, C., Oporto, G. S., Zhong, T. \& Jaczynski, J. TEMPO nanofibrillated cellulose as template for controlled release of antimicrobial copper from PVA films. Cellulose 23, 713722 (2016).

13. Zhong, T. et al. Antimicrobial Properties of the Hybrid Copper NanoparticlesCarboxymethyl Cellulose. Wood Fiber Sci. 45, 215-222 (2013).

14. Wang, G. et al. parallel evolution between dogs and humans. Nat. Commun. 4, 1860-1869 (2013).

15. Habibi, Y., Lucia, L. A. \& Rojas, O. J. Cellulose nanocrystals: Chemistry, self-assembly, and applications. Chem. Rev. 110, 3479-3500 (2010).

16. Padalkar, S. et al. Natural biopolymers: Novel templates for the synthesis of nanostructures. Langmuir 26, 8497-8502 (2010).

17. Shi, Z. et al. In situ nano-assembly of bacterial cellulose-polyaniline composites. RSC Adv. 2, 1040-1046 (2012).

18. Cioffi, N., Ditaranto, N., Torsi, L. \& Sabbatini, L. Approaches to Synthesis and Characterization of Spherical and Anisotropic Copper Nanomaterials. Nanotechnologies for the Life Sciences (2010). doi:10.1002/9783527610419.ntls0123

19. Owens, C. W., Oporto, G. S., Söderberg, B. C. G. \& Lambson, K. E. Lignocellulosic Microand Nanomaterials as Copper Frames for the Evaluation of the Copper(I)-Catalyzed Azide- 
Alkyne Cycloaddition. J. Nanomater. 2017, (2017).

20. Hassanzadeh, M. et al. Nanofibrillated Cellulose from Appalachian Hardwoods Logging Residues as Template for Antimicrobial Copper. J. Nanomater. 2017, (2017).

21. Ingle, A. P., Duran, N. \& Rai, M. Bioactivity, mechanism of action, and cytotoxicity of copper-based nanoparticles: A review. Appl. Microbiol. Biotechnol. 98, 1001-1009 (2014).

22. Hostynek, J. J. \& Maibach, H. I. Copper hypersensitivity: dermatologic aspects. Dermatol. Ther. 17, 328-333 (2004).

23. Borkow, G. \& Gabbay, J. Putting copper into action: copper-impregnated products with potent biocidal activities. FASEB J. 18, 1728-1730 (2004).

24. Gerstein, A. C. et al. Too much of a good thing: the unique and repeated paths toward copper adaptation. Genetics 199, 555-71 (2015).

25. Sparks, D. L. \& Schreurs, B. G. Trace amounts of copper in water induce beta-amyloid plaques and learning deficits in a rabbit model of Alzheimer's disease. Proc. Natl. Acad. Sci. U. S. A. 100, 11065-11069 (2003).

26. Xiao, G., Fan, Q., Wang, X. \& Zhou, B. Huntington disease arises from a combinatory toxicity of polyglutamine and copper binding. Proc Natl Acad Sci U S A 110, 14995-15000 (2013).

27. Rademacher, C. \& Masepohl, B. Copper-responsive gene regulation in bacteria. Microbiol. (United Kingdom) 158, 2451-2464 (2012).

28. Robinson, N. J. \& Winge, D. R. Copper Metallochaperones. Annu. Rev. Biochem. 79, $537-$ 562 (2010).

29. Rutherford, J. C. \& Bird, A. J. Metal-Responsive Transcription Factors That Regulate Iron, Zinc, and Copper Homeostasis in Eukaryotic Cells. Eukaryot. Cell 3, 1-13 (2004).

30. Wang, Y., Hodgkinson, V., Zhu, S., Weisman, G. A. \& Petris, M. J. Advances in the Understanding of Mammalian Copper Transporters. Adv. Nutr. 2, 129-137 (2011).

31. Solomon, E. I. et al. Copper Active Sites in Biology. Chem. Rev. 7, 3659-3853 (2014).

32. Nevitt, T., Öhrvik, H. \& Thiele, D. J. Charting the travels of copper in eukaryotes from yeast to mammals. Biochim. Biophys. Acta - Mol. Cell Res. 1823, 1580-1593 (2012).

33. Turski, M. L. et al. A Novel Role for Copper in Ras/Mitogen-Activated Protein Kinase Signaling. Mol. Cell. Biol. 32, 1284-1295 (2012).

34. Georgatsou, E. \& Alexandraki, D. Two distinctly regulated genes are required for ferric reduction, the first step of iron uptake in Saccharomyces cerevisiae. Mol. Cell. Biol. 14, 3065-3073 (1994).

35. Georgatsou, E., Mavrogiannis, L. A., Fragiadakis, G. S. \& Alexandraki, D. The yeast Fre 1p/Fre2p cupric reductases facilitate copper uptake and are regulated by the coppermodulated Mac1p activator. J. Biol. Chem. 272, 13786-13792 (1997).

36. Jungmann, J. et al. $\mathrm{MAC1}$, a nuclear regulatory protein related to $\mathrm{Cu}$-dependent transcription factors is involved in $\mathrm{Cu} / \mathrm{Fe}$ utilization and stress resistance in yeast. EMBOJ. 12, 5051-5056 (1993).

37. Dancis, A., Haile, D., Yuan, D. S. \& Klausnert, R. D. The Saccharomyces cerevisiae Copper Transport Protein (Ctr1p). J. Biol. Chem. 269, 25660-25667 (1994).

38. Hassett, R. \& Kosman, D. J. Evidence for Cu(II) reduction as a component of copper uptake by Saccharomyces cerevisiae. Journal of Biological Chemistry 270, 128-134 (1995).

39. Labbé, S., Zhu, Z. \& Thiele, D. J. Copper-specific transcriptional repression of yeast genes encoding critical components in the copper transport pathway. J. Biol. Chem. 272, 1595115958 (1997). 
40. Liu, J., Sitaram, A. \& Burd, C. G. Regulation of copper-dependent endocytosis and vacuolar degradation of the yeast copper transporter, Ctr1p, by the Rsp5 ubiquitin ligase. Traffic $\mathbf{8}$, 1375-1384 (2007).

41. Thiele, D. J. ACE1 regulates expression of the Saccharomyces cerevisiae metallothionein gene. Mol. Cell. Biol. 8, 2745-2752 (1988).

42. Gralla, E. B., Thiele, D. J., Silar, P. \& Valentine, J. S. ACE1, a copper-dependent transcription factor, activates expression of the yeast copper, zinc superoxide dismutase gene. Proc. Natl. Acad. Sci. U. S. A. 88, 8558-8562 (1991).

43. Culotta, V. C., Howard, W. R. \& Liu, X. F. CRS5 encodes a metallothionein-like protein in Saccharomyces cerevisiae. J. Biol. Chem. 269, 25295-25302 (1994).

44. Nikko, E. \& Pelham, H. R. B. Arrestin-mediated endocytosis of yeast plasma membrane transporters. Traffic 10, 1856-1867 (2009).

45. Lin, C. H., MacGurn, J. A., Chu, T., Stefan, C. J. \& Emr, S. D. Arrestin-Related UbiquitinLigase Adaptors Regulate Endocytosis and Protein Turnover at the Cell Surface. Cell 135, 714-725 (2008).

46. Polo, S. et al. A single motif responsible for ubiquitin recognition and monoubiquitination in endocytic proteins. Lett. to Nat. 416, 451-455 (2002).

47. Reider, A. \& Wendland, B. Endocytic adaptors - Social networking at the plasma membrane. J. Cell Sci. 124, 1613-1622 (2011).

48. Shih, S. C. et al. Epsins and Vps27p/Hrs contain ubiquitin-binding domains that function in receptor endocytosis. Nat. Cell Biol. 4, 389-393 (2002).

49. Moore, C. A. C., Milano, S. K. \& Benovic, J. L. Regulation of Receptor Trafficking by GRKs and Arrestins. Annu. Rev. Physiol. 69, 451-482 (2007).

50. Lefkowitz, R. J., Rajagopal, K. \& Whalen, E. J. New Roles for $\beta$-Arrestins in Cell Signaling: Not Just for Seven-Transmembrane Receptors. Mol. Cell 24, 643-652 (2006).

51. Szabó, E. Z., Numata, M., Lukashova, V., Iannuzzi, P. \& Orlowski, J. $\beta$-arrestins bind and decrease cell-surface abundance of the $\mathrm{Na}+\mathrm{H}+$ exchanger NHE5 isoform. Proc. Natl. Acad. Sci. U. S. A. 102, 2790-2795 (2005).

52. Palza, H. Antimicrobial polymers with metal nanoparticles. Int. J. Mol. Sci. 16, 2099-2116 (2015).

53. Lemire, J. A., Harrison, J. J. \& Turner, R. J. Antimicrobial activity of metals: Mechanisms, molecular targets and applications. Nat. Rev. Microbiol. 11, 371-384 (2013).

54. Lloyd, R. V, Hanna, P. M. \& Mason, R. P. The origin of the hydroxyl radical oxygen in the Fenton reaction. Free Radic. Biol. Med. 22, 885-888 (1997).

55. Manzl, C., Enrich, J., Ebner, H., Dallinger, R. \& Krumschnabel, G. Copper-induced formation of reactive oxygen species causes cell death and disruption of calcium homeostasis in trout hepatocytes. Toxicology 196, 57-64 (2004).

56. Grass, G., Rensing, C. \& Solioz, M. Metallic copper as an antimicrobial surface. Appl. Environ. Microbiol. 77, 1541-1547 (2011).

57. Apel, K. \& Hirt, H. REACTIVE OXYGEN SPECIES: Metabolism, Oxidative Stress, and Signal Transduction. Annu. Rev. Plant Biol. 55, 373-399 (2004).

58. Pearson, R. G. Hard and soft acids and bases. J. Ameri. Chem. Soc. 3533-9 (1963).

59. Parr, R. G. \& Pearson, R. G. Absolute Hardness: Companion Parameter to Absolute Electronegativity. J. Am. Chem. Soc. 105, 7512-7516 (1983).

60. Foster, A. W. \& Robinson, N. J. Promiscuity and preferences of metallothioneins: The cell rules. BMC Biol. 9, 25-27 (2011). 
61. Workentine, M. L., Harrison, J. J., Stenroos, P. U., Ceri, H. \& Turner, R. J. Pseudomonas fluorescens' view of the periodic table. Environ. Microbiol. 10, 238-250 (2008).

62. Nies, D. H. Efflux-mediated heavy metal resistance in prokaryotes. FEMS Microbiol. Rev. 27, 313-339 (2003).

63. Harrison, J. J., Ceri, H. \& Turner, R. J. Multimetal resistance and tolerance in microbial biofilms. Nat. Rev. Microbiol. 5, 928-938 (2007).

64. Postma, E., Verduyn, C., Scheffers, A. \& Van Dijken, johannes P. Enzymic analysis of the Crabtree Effect in glucose-limited chemostat cultures of Saccharomyces cerevisiae. Appl. Environ. Microbiol. 55, 468-477 (1989).

65. Pronk, J. T., Yde Steensma, H. \& Van Dijken, J. P. Pyruvate Metabolism in Saccharomyces cerevisiae. Yeast 12, 1607-1633 (1996).

66. Hagman, A. \& Piškur, J. A study on the fundamental mechanism and the evolutionary driving forces behind aerobic fermentation in yeast. PLoS One 10, 1-24 (2015).

67. Galdieri, L., Mehrotra, S., Yu, S. \& Vancura, A. Transcriptional regulation in yeast during diauxic shift and stationary phase. Omi. A J. Integr. Biol. 14, 629-638 (2010).

68. Thomson, J. M. et al. Resurrecting ancestral alcohol dehydrogenases from yeast. Nat. Genet. 37, 630-5 (2005).

69. Wolfe, K. H. \& Shields, D. C. Molecular evidence for an ancient duplication of the entire yeast genome. Nature 387, 708-713 (1997).

70. Ihmels, J. et al. Rewiring of the Yeast Transcriptional Network Through the Evolution of Motif Usage Rewiring of the Yeast Transcriptional Network Through the Evolution of Motif Usage. Science (80-. ). 309, 938-940 (2005).

71. Martini, A. V. \& Martini, A. Three newly delimited species of Saccharomyces sensu stricto. Antonie Van Leeuwenhoek 53, 77-84 (1987).

72. Wang, S. A. \& Bai, F. Y. Saccharomyces arboricolus sp. nov., a yeast species from tree bark. Int. J. Syst. Evol. Microbiol. 58, 510-514 (2008).

73. Naumova, E. S., Roberts, I. N., James, S. A., Naumov, G. I. \& Louis, E. J. Three new species in the Saccharomyces sensu stricto complex: Saccharomyces cariocanus, Saccharomyces kudriavzevii and Saccharomyces mikatae. Int. J. Syst. Evol. Microbiol. 50, 1931-1942 (2015).

74. Naumov, G. I., Naumova, E. S., Hagler, A. N., Mendonça-Hagler, L. C. \& Louis, E. J. A new genetically isolated population of the Saccharomyces sensu stricto complex from Brazil. Antonie Van Leeuwenhoek 67, 351-355 (1995).

75. Naumov, G. I., Lee, C.-F. \& Naumova, E. S. Molecular genetic diversity of the Saccharomyces yeasts in Taiwan: Saccharomyces arboricola, Saccharomyces cerevisiae and Saccharomyces kudriavzevii. Antonie Van Leeuwenhoek 103, 217-28 (2013).

76. Naumov, G. I., Naumova, E. S. \& Louis, E. J. Two New Genetically Isolated Popoulations of the Saccharomyces Sensu Stricto Complex from Japan. J. Gen. Appl. Microbiol. 41, 499505 (1995).

77. Libkind, D. et al. Microbe domestication and the identification of the wild genetic stock of lager-brewing yeast. Proc. Natl. Acad. Sci. U. S. A. 108, 14539-44 (2011).

78. Boynton, P. J. \& Greig, D. The ecology and evolution of non-domesticated Saccharomyces species Primrose. Yeast 31, 449-462 (2014).

79. Sipiczki, M. Interspecies hybridisation and genome chimerisation in Saccharomyces: Combining of gene pools of species and its biotechnological perspectives. Front. Microbiol. 9, 1-20 (2018). 
80. Cliften, P. et al. Finding functional features in Saccharomyces genomes by phylogenetic footprinting. Science (80-. ). 301, 71-76 (2003).

81. Birren, B., Lander, E. S., Kellis, M., Endrizzi, M. \& Patterson, N. Sequencing and comparison of yeast species to identify genes and regulatory elements. Nature 423, 241254 (2003).

82. Dujon, B. Yeasts illustrate the molecular mechanisms of eukaryotic genome evolution. Trends Genet. 22, 375-387 (2006).

83. Gayevskiy, V. \& Goddard, M. R. Saccharomyces eubayanus and Saccharomyces arboricola reside in North Island native New Zealand forests. Environ. Microbiol. 18, 1137-1147 (2016).

84. Liti, G. et al. High quality de novo sequencing and assembly of the Saccharomyces arboricolus genome. BMC Genomics 14, 14:69 (2013).

85. Masneuf, I., Hansen, J., Groth, C., Piskur, J. \& Dubourdieu, D. New hybrids between Saccharomyces sensu stricto yeast species found among wine and cider production strains. Appl. Environ. Microbiol. 64, 3887-3892 (1998).

86. Querol, A. \& Bond, U. The complex and dynamic genomes of industrial yeasts: MINIREVIEW. FEMS Microbiol. Lett. 293, 1-10 (2009).

87. Nguyen, H. V., Legras, J. L., Neuvéglise, C. \& Gaillardin, C. Deciphering the hybridisation history leading to the lager lineage based on the mosaic genomes of Saccharomyces bayanus strains NBRC1948 and CBS380 T. PLoS One 6, (2011). 


\title{
Proteomic and Genetic Analysis of the Response of Saccharomyces cerevisiae to Soluble Copper Leads to Improvement of the Antimicrobial Function of Cellulosic Copper Nanoparticles
}

An original research article published in Metallomics from the Royal Society of Chemistry Reprinted by permission from the Royal Society of Chemistry: Metallomics, 9: 1304-1315

Xiaoqing Rong-Mullins $\ddagger$, Matthew J. Winans $\$$, Justin B. Lee, Zachery R. Lonergan, Vincent A. Pilolli, Lyndsey M. Weatherly, Thomas W. Carmenzind, Lihua Jiang, Jonathan R. Cumming, Gloria Oporto, Jennifer E.G. Gallagher

$\$$ These authors contributed equally

\begin{abstract}
Copper $(\mathrm{Cu})$ was used in antiquity to prevent waterborne and food diseases because, as a broadspectrum antimicrobial agent, it generates reactive oxygen species, ROS. New technologies incorporating $\mathrm{Cu}$ into low-cost biodegradable nanomaterials built on cellulose, known as cellulosic cupric nanoparticles or c-CuNPs, present novel approaches to deliver $\mathrm{Cu}$ in a controlled manner to control microbial growth. We challenged strains of Saccharomyces cerevisiae to soluble $\mathrm{Cu}$ and c-CuNPs to evaluate the potential of c-CuNPs as antifungal agents. Cells exposed to c-CuNPs demonstrated greater sensitivity to $\mathrm{Cu}$ than cells exposed to soluble $\mathrm{Cu}$, although $\mathrm{Cu}$-resistant strains were more tolerant than $\mathrm{Cu}$-sensitive strains of c-CuNP exposure. At the same level of growth inhibition, $157 \mu \mathrm{M}$ c-CuNP led to the same internal $\mathrm{Cu}$ levels as did $400 \mathrm{CuSO}_{4}$, offering evidence for alternative mechanisms of toxicity, perhaps through $\beta$-arrestin dependent endocytosis, which was supported by flow cytometry and fluorescence microscopy of c-CuNPs distributed both on the cell surface and within the cytoplasm. Genes responsible for genetic variation to copper were mapped to the ZRT2 and the CUP1 loci. Through proteomic analyses, we found that the expression of other zinc $(\mathrm{Zn})$ transporters increased in $\mathrm{Cu}$-tolerant yeast compared to $\mathrm{Cu}$-sensitive strains. Further, the addition of $\mathrm{Zn}$ at low levels increased the potency of c-CuNP to inhibit even the most $\mathrm{Cu}$-tolerant yeast. Through unbiased systems biological approaches, we identified $\mathrm{Zn}$ as a critical component of yeast response to $\mathrm{Cu}$ and the addition of $\mathrm{Zn}$ increased potency of the cCuNPs.
\end{abstract}




\section{Introduction}

Copper $(\mathrm{Cu})$ has been used since ancient times to prevent microbial growth because of its broadspectrum activity coupled with multiple modes of toxicity ${ }^{1}$. Excessive $\mathrm{Cu}$ induces damage by catalyzing the generation of reactive oxygen species (ROS) ${ }^{2}$. ROS generated by extracellular $\mathrm{Cu}$ oxidizes lipids and proteins of the cell membrane ${ }^{3}$ and, if cells survive these lesions, then DNA damage can occur from the high levels of internal $\mathrm{Cu}^{4}$. These mechanisms of toxicity and the response to $\mathrm{Cu}$ in microorganisms have been widely studied ${ }^{5}$. Importantly, the expression of genes in response to $\mathrm{Cu}$ is highly regulated to maintain a fine balance of this essential, yet toxic, trace metal within the cytoplasm 6,7 .

Nanotechnology is a transformative tool that can be used to develop and enhance high-value products from renewable and biocompatible raw materials such as $\operatorname{wood}^{8-12}$. Cellulose, as the predominant component of woody biomass, can be utilized not only commercially where biocompatibility and biodegradability are relevant, but also as a support structure for nanoparticle formation in novel applications. The use of the cellulose nanostructure as a template and stabilizer for metallic nanoparticles expands the potential applications of nanomaterials ${ }^{13}$. Cellulosic cuprous nanoparticles (c-CuNPs) display effective antimicrobial activity against a non-pathogenic Escherichia coli DH5 $\alpha^{14,15}$. c-CuNPs contain reduced copper $(\mathrm{Cu}(\mathrm{I}))$, which is thought to be slowly released as $\mathrm{Cu}(\mathrm{I})$ is oxidized making $\mathrm{Cu}(\mathrm{II})$ available to interact with biological molecules leading to toxicity.

Improving c-CuNPs depends on understanding its range of functions in order to manufacture products with increased antimicrobial effectiveness. We have developed a cost-effective hybrid nanomaterial built on a cellulosic scaffold with $\mathrm{Cu}(\mathrm{I})$ chemically linked to cellulose ${ }^{16}$. To understand how yeast respond to $\mathrm{Cu}$ delivered in $\mathrm{c}-\mathrm{CuNPs}$, we carried out toxicological and quantitative proteomic analyses on genetically diverse yeast strains. c-CuNPs delivered more $\mathrm{Cu}$ to yeast cells compared to soluble $\mathrm{CuSO}_{4}$, and this may result from the endocytic uptake of c$\mathrm{CuNPs}$. The levels of hundreds of proteins changed in response to $\mathrm{Cu}$ exposure, and yeast strains that do not tolerate $\mathrm{Cu}$ fail to increase expression of $\mathrm{Zrt1}$, a high affinity zinc ( $\mathrm{Zn}$ ) transporter. Further, a genome-wide quantitative trait loci (QTL) analysis uncovered a role for Zn transporters in $\mathrm{Cu}$ response. The genetic variation in the low-affinity membrane transporter, Zrt2, directly contributed to variation of $\mathrm{Cu}$ tolerance, while yeast lacking Zrt1 or Zrt3 were sensitive to high levels of $\mathrm{Cu}$. Supplementing $\mathrm{Zn}$ decreased growth of all yeast strains tested when also challenged with c-CuNPs. Understanding the interdependence of metal tolerances provides insights into improving antimicrobial properties of metal-based materials.

\section{Materials and Methods}

\section{Yeast Strains and Plasmids}

S288c derived strains used included strains S96 (MATa lys $5^{17}$ ) and GSY147 (MATa prototroph ${ }^{18}$ ). YJM789 (MATalpha $l y s 2^{17,19}$ ) was the parent of YJM789K5a (MATa prototroph). YJM789K5a was generated by transforming a PCR product containing LYS2 into YJM789 and then backcrossed to HS959 (MATa lys2) that is otherwise isogenic to YJM789. All S288c strains displayed the similar copper resistance. Both YJM789 derived strains were copper sensitive. Other strains used 
were AWRI1631, RM11, and YJM33920-22. The haploid recombinant segregant collection between YJM789 and S96 was previously generated ${ }^{17}$. Knockout strains were previously described $^{23}$. Cloning of ZRT2 alleles was done with PCR amplified from genomic DNA using primers as follows 5'ZRT2 GTACCTGCAGGCGTCCTAATGAAAATGGAGG and 3'ZRT2 TTCCCGGGATTACTCTGCCCTTTTC. Endogenous promoter and terminator sequences within 580 nucleotides upstream of the start and 277 nucleotides downstream of the stop codons were included. Genes were cloned into the SbfI and XmaI sites of pGS36 plasmid with the hygromycin resistance marker ${ }^{18}$. Plasmids were kept under selection with hygromycin after LiAc chemical transformations.

\section{Media and Chemicals}

All haploid yeast strains were grown in yeast minimal media (YM), which includes $2 \%$ dextrose, $6.7 \mathrm{~g} / \mathrm{L}$ yeast nitrogen base, and $20 \mathrm{~g} / \mathrm{L}$ agar in solid media. In liquid media, the agar was omitted. YM was, when needed, supplemented with lysine, methionine, uracil, leucine, and histidine. Plasmids were maintained by addition of $250 \mu \mathrm{g} / \mathrm{ml}$ hygromycin. All antioxidants were added to solid media when it had cooled to $55^{\circ} \mathrm{C}$ and used within 24 hours. Chronic exposure was defined as two days of exposure, while acute exposure was defined as two hours of exposure. Viability assays were done in liquid culture in biological triplicates. Yeast were grown to log-phase and split into subcultures. Subcultures served as controls, receiving no added $\mathrm{Cu}$; soluble $\mathrm{Cu}$-treated, receiving various concentrations of $\mathrm{CuSO}_{4} \cdot 5 \mathrm{H}_{2} \mathrm{O}$ (Ricco Chemicals, CAS 7732-18-5); or receiving c-CuNPs. Carboxymethyl cellulose (CMC) copper nanoparticles (c-CuNPs) were created and physically characterized as previously reported ${ }^{16}$, where $\mathrm{Cu}$ is reduced and bound onto free hydroxyls of the cellulose scaffold. $10 \mu \mathrm{g} / \mathrm{ml}$ of c-CuNP corresponded to $157 \mu \mathrm{M}$ of soluble $\mathrm{Cu}$ determined by atomic absorption spectrometry (AAS). Yeast media are acidic ( $\mathrm{pH} 5$ ), which would cause dissociation and oxidization of $\mathrm{Cu}$ from the c-CuNPs. Indeed, 27\% more free copper was found in $\mathrm{c}-\mathrm{CuNPs}$ suspended in YM than in water alone.

\section{Atomic Absorption Spectrometry}

Strains YJM789K5a, GSY147, RM11, and YJM339 were grown in YM medium in triplicate into log phase. The control samples (YM) were collected. The remaining cultures were treated with $400 \mu \mathrm{M} \mathrm{CuSO}_{4}$ or $157 \mu \mathrm{M} \mathrm{Cu}$ as c-CuNP $(10 \mu \mathrm{g} / \mathrm{ml}$ of CMC) for 2 hours. For cell collection, equal number of cells, as determined by optical density at $600 \mathrm{~nm}$, was obtained (equivalent to 4 $\mathrm{ODu}$ ). These samples were centrifuged and washed twice with distilled water. One ODu was separated for protein quantification. Samples were split, frozen in liquid nitrogen and stored at $80^{\circ} \mathrm{C}$. Soluble protein concentration was determined via Bradford assay. For AAS, the cell pellets were treated with $600 \mu \mathrm{l}$ of concentrated $\mathrm{HNO}_{3}$ and $200 \mu \mathrm{l}$ of $30 \% \mathrm{H}_{2} \mathrm{O}_{2}$ for digestion. These solutions were transferred to glass tubes and placed in a boiling water bath for 2 hours until clear. A 1:10 dilution with distilled water was analyzed for $\mathrm{Cu}$ at $324.8 \mathrm{~nm}$. Copper concentrations were given in $\mathrm{mg} / \mathrm{L}$, adjusted for dilution, and normalized to soluble protein concentration for each sample. AAS was performed across four days, and a total average for the four repeated samples was determined for each day. From these values, the overall average was determined. To calculate the correction factor for each day's analysis, the overall average was divided by the daily average. This correction factor was then applied to all the values for that particular day, prior to dilution adjustments. 


\section{Staining of Yeast Cells and Cellulose Copper Nanoparticles for Flow Cytometry and Microscopy}

Cells of each strain were cultured from frozen stock in YM media to log phase and harvested by centrifugation. Cells were then transferred to $10 \mathrm{mM}$ 4-(2-hydroxyethyl)-1piperazineethanesulfonic acid (HEPES) buffer ( $\mathrm{pH}$ of 7.4) containing 5\% dextrose. Rhodamine B was added to a final concentration of $100 \mathrm{nM}$, and cells were incubated at room temperature for 20 minutes in the dark. c-CuNPs were added to phosphate buffered saline (PBS) to a concentration of $400 \mu \mathrm{M}$ with $5.136 \mathrm{mM}$ FITC in dimethyl sulfoxide (DMSO) to a final concentration of 2.568 $\mu \mathrm{M}$. The FITC-dyed PBS nanoparticle solution was incubated in the dark for 8 hours at $4^{\circ} \mathrm{C}$. Because excess FITC could not be removed from the solution, $5.136 \mathrm{mM}$ in DMSO was added to PBS to a final concentration of $2.568 \mu \mathrm{M}$ and incubated in the dark for 8 hours at $4^{\circ} \mathrm{C}$ to serve as a control.

Fluorescein isothiocyanate (FITC), HEPES, Rhodamine B Hexyl Ester (RhodB), and dextrose were purchased from Fisher Scientific (Chicago, IL). DMSO was purchased from Biotium (Hayward, CA). A four laser LSR/Fortessa (BD Biosciences, San Jose CA) with BD FACS Diva software (BD Biosciences) located in the WVU Flow Cytometry \& Single Cell Core Facility was used to collect flow cytometric data. Cells of each strain were transferred from HEPES buffer, treated, and transferred to PBS. To determine the extent of association of c-CuNPs with the yeast cell wall, $1 \mathrm{~mL}$ of PBS containing 1 million dyed cells was added to $1 \mathrm{~mL}$ of $400 \mu \mathrm{m}$ dyed c-CuNPs PBS solution. To control for FITC labeling of cells, $1 \mathrm{~mL}$ of PBS containing 1 million dyed cells was added to $1 \mathrm{~mL}$ of $2.568 \mu \mathrm{M}$ FITC in PBS. Solutions were allowed to incubate at $30^{\circ} \mathrm{C}$ for 2 hours. Samples in PBS were analyzed on an LSR Fortessa (BD Biosciences San Jose CA) using BD FACS Diva version 8.0 software (Fig 2-S1). A total of 10,000 events were collected/sample and the median fluorescence intensity for each sample was calculated. Also, in order to ensure that the FITC signal only came from c-CuNPs that were inside the yeast, trypan blue was added to the samples prior to analysis on the flow cytometer.

After treatment, samples were harvested by centrifugation and re-suspended in $1 \mathrm{ml}$ PBS containing $4 \%$ paraformaldehyde and incubated for 30 minutes at $30^{\circ} \mathrm{C}$. Cells were washed twice with PBS and re-suspended in $500 \mu \mathrm{l} 80 \%$ glycerol before sonicating at low for 5 intervals of 1 second bursts on a Branson model SSE-1 Sonifier. Concanavalin A (Sigma - L7647) coated microscope slides were utilized to mount $10 \mu \mathrm{l}$ of the samples and sealed with clear fingernail polish. Fluorescent yeasts were viewed with a laser scanning confocal microscope (Olympus FV1000) equipped with red/green HeNe and argon lasers. Images were acquired using a 60x oil immersion magnification optical objective. Distances between confocal planes were optimized for the objective using Fluoview software (FV10-ASW Version 04.00.02.09). Pixel resolution was adjusted for best resolution capable of performing a z-stack scan. Images were only modified for contrast enhancement.

\section{Viability Assay}

After two hours with $400 \mu \mathrm{M}$ of copper or $157 \mu \mathrm{M}$ c-CuNPs, cultures were washed twice with water, and the $\mathrm{OD}_{600}$ was measured. 0.1 OD units (ODu) of each sample were diluted 100-fold and $100 \mu 1$ plated on YPD. The number of untreated colonies that grew were considered $100 \%$ viability, and the viability of treated cells were taken as a percentage of untreated cells. 


\section{Proteomics}

To measure the global proteomic changes of S288c and YJM789 in response to Cu, cells were treated for 90 minutes in YM with or without $800 \mu \mathrm{M} \mathrm{CuSO}_{4}$. Peptides were prepared using a modified FASP method ${ }^{24}$. Treated cells were harvested by briefly spinning down 5 ODu of cells, washing once with water and then freezing cell pellets in liquid nitrogen in $2 \mathrm{~mL}$ screw cap tubes. Cells were lysed in 4\% SDS, $100 \mathrm{mM}$ DTT, and $100 \mathrm{mM}$ Tris $\mathrm{pH} 8.5$ in a 1:10 v/v cell pellet to buffer. $0.5 \mathrm{~mm}$ zirconium beads were added and cells were lysed in a Fastprep at $3 \times 20$ seconds at speed 5 with a 5 -minute rest at room temperature. Using a 24-gauge needle, the bottom of the tube was punctured and placed into $2 \mathrm{ml}$ low protein binding locking cap tubes. All liquid and cellular debris were then spun at $1000 \mathrm{rpm}$ into a $2-\mathrm{ml}$ tube. Tubes were incubated at $95^{\circ} \mathrm{C}$ for 4 minutes. Cellular debris was pelleted for 10 minutes at $14,000 \mathrm{rpm}$ at room temperature. The supernatant was transferred to new tubes. Protein concentration was calculated using Bradford reagent on 1:100 diluted sample. $200 \mu \mathrm{g}$ of protein was diluted with $8 \mathrm{M}$ urea/100 mM Tris $\mathrm{pH} 8.5$ to a final SDS concentration of $1 \%$ and transferred to a Microcon YM-30 column. Microcon columns were spun at 10,000 rpm. Proteins were washed five times with $8 \mathrm{M}$ urea/100 mM Tris $\mathrm{pH} 8.5$ and resuspended in $100 \mu 10.05 \mathrm{M}$ idoacetamide in $8 \mathrm{M}$ urea/0.1 M Tris $\mathrm{HCl}$ 8.5. Samples were alkylated for 45 minutes in the dark. Liquid was spun through the Microcon. Buffer was exchanged into $200 \mathrm{mM}$ TEAB by washing proteins five times. Protein concentrations were retested using a BCA kit (Thermo Fisher Scientific) on 1:100 diluted samples. Trypsin was freshly dissolved in $200 \mathrm{mM}$ TEAB and incubated at $30^{\circ} \mathrm{C}$ for 20 minutes. Trypsin was incubated with proteins on the Microcon wrapped in parafilm overnight at $37^{\circ} \mathrm{C}$ at 1:20 dilution. Digested peptides were spun through the Microcon and collected into fresh tubes. Concentration of peptides were approximated, and $5 \mu \mathrm{g}$ of peptides were labeled with Thermo Fisher Tandem Mass Tags 6plex in a 10:4.1 ratio according to manufacturer's instructions for 1.5 hours at room temperature. The reaction was quenched with 5\% hydroxylamine diluted in $200 \mathrm{mM}$ TEAB. Equal amounts of labeled peptides were mixed and dried.

Peptides were resuspended in $100 \mathrm{mM}$ ammonia formate at $\mathrm{pH} 10$ and separated by reverse phase chromatography at high $\mathrm{pH}$ in the first dimension, followed by an orthogonal separation at low $\mathrm{pH}$ in the second dimension. In the first dimension the mobile phases were buffer $A: 20 \mathrm{mM}$ ammonium formate at $\mathrm{pH} 10$ and buffer B: Acetonitrile. Peptides were separated on a Xbridge $300 \mu \mathrm{m} \times 5 \mathrm{~cm} \mathrm{C} 185.0 \mu \mathrm{m}$ column (Waters) using 15 discontinuous step gradient at $2 \mu 1 / \mathrm{min}$. Peptides eluted off from the first dimension were trapped by Symmetry $180 \mu \mathrm{m}$ x $2 \mathrm{~cm} \mathrm{C18} 5.0 \mu \mathrm{m}$ trap column (Waters). In the second dimension, peptides were loaded to an in-house packed 75 $\mu \mathrm{m}$ ID $/ 15 \mu \mathrm{m}$ tip ID $\times 20 \mathrm{~cm}$ C18-AQ $3.0 \mu \mathrm{m}$ resin column with buffer A $(0.1 \%$ formic acid in water). Peptides were separated with a linear gradient from $5 \%$ to $30 \%$ buffer B $(0.1 \%$ formic acid in acetonitrile) at a flow rate of $300 \mathrm{nl} / \mathrm{min}$ over 180 minutes. Each sample separation was repeated three times.

Peptides were identified by a linear trap quadrupole (LTQ)-Orbitrap Velos instrument (Thermo Fisher Scientific). The mass spectrometer was run in a data dependent mode. One survey scan acquired in the Orbitrap mass analyzer with resolution 60,000 was followed by MS/MS of the 10 most intense peaks with charge state $\geq 2$ and above an intensity threshold of 5,000. Peptides were fragmented in the high collisional Cell (HCD) with normalized collision energy of $40 \%$ and activation time of $0.1 \mathrm{~s}$. The MS/MS scan was acquired in the Orbitrap at resolution of 7,500. 
Peptides were identified and quantitated using Proteome Discoverer 1.4.1.14 (2-S1 Table). Spectra were searched against a protein database consisting of all the S288c (http://downloads.yeastgenome.org/sequence/S288C_reference/genome_releases/S288C_referen ce_genome_R64-1-1_20110203.tgz) and YJM789 (http://downloads.yeastgenome.org/sequence/strains/YJM789/YJM789_Stanford_2007_AAFW0 2000000) proteins available on Saccharomyces Genome Database (http://www.yeastgenome.org). Common contaminants such as human keratin and trypsin were also included in the database to prevent peptides of those contaminants from being falsely identified as yeast peptides (2-S2 Table). TMT6plex was used for quantification labeling according to manufacturer's instructions and the six isobaric labels of 126-131 Da were assigned to samples as follows: 126 - S288c, YM, replicate 1; 127 - S288c, YM, replicate 2; 128 - S288c, Cu treated, replicate 1; 129 - S288c, Cu treated, replicate 1;130 - YJM789, YM; 131 - YJM789, Cu treated.

The default method used by Proteome Discoverer to quantify protein fold change from peptide intensities is to calculate the median intensity ratio between two isobaric labels for all the peptide spectrum matches (PSMs) associated with the protein in question. This method is problematic when a peptide sequence is identified in multiple PSMs where ratios have large variation. For example, a peptide can have different phosphorylation states between two treatments, and consequently show very different ratios for the phosphorylated PSMs and the unphosphorylated PSMs. This type of variation can skew quantification results when there are only a few PSMs usable for quantification; therefore, we used different methods than the Proteome Discoverer default for protein quantification. For each peptide sequence identified by Proteome Discoverer, the reporter ion intensities of all PSMs associated with this peptide were summed into the total intensity for this peptide in each sample. For each protein, only its unique (not belonging to any other protein) peptides of high to middle confidence (false discovery rate $0.01-0.05$ ) were considered usable for quantification. Four comparisons, each between two experimental scenarios, were performed by calculating intensity ratios of reporter ions: (1) S288c, Cu vs. YM - (128+ 129) / (126 + 127); (2) YJM789, Cu vs. YM - 131/130; (3) YJM789 vs. S288c, YM - $130 \times 2$ / $(126+127)$; (4) YJM789 vs. S288c, $\mathrm{Cu}-131 \times 2 /(128+129)$. For the comparisons between strains, (3) and (4), only peptides showing no sequence polymorphisms between the two strains were considered usable for quantification. Four methods were used to calculate protein ratios for each comparison: (1) 'means then ratio' - the mean of all the usable peptides belonging to this protein was calculated for each experimental scenario, then the ratio between the two scenarios was calculated from the two means; (2) 'medians then ratio' - the median of usable peptides was calculated for each scenario, then the ratio was calculated from two medians; (3) 'ratios then mean' - the ratio between the two scenarios was calculated for each usable peptide, then the mean of all the ratios was calculated; (4) 'ratios then median' - ratio was calculated for each usable peptide, then the median of all the ratios was calculated. For each of the four methods and each comparison of two scenarios, the resulting ratios for all the proteins were divided by the median of the ratios for normalization. This normalization method assumes that the majority of reported proteins do not change much in each comparison, which was the case in our experiment (see Results). For each comparison, only the proteins showing ratios $>2$ or $<0.5$ by all the four methods were included in Table 2-S1. The ratios calculated using method (1) 'means then ratio' were transformed into logarithms of 2 (two). 
63,563 PSMs were reported with high to middle confidence (false discovery rate 0.01-0.05), representing 27,794 unique peptide sequences. Peptides were labeled with isobaric mass tags for quantification with Tandem Mass Tags (TMT). TMT labeling rates of those PSMs were 92.7$97.3 \%$. 4,431 yeast proteins were reported containing at least one peptide with middle to high confidence (each case of two homologous proteins from S288c and YJM789 were counted as one protein), among which 2,991 were quantified with at least two unique peptides and considered for differential abundance quantification. 112 proteins showed greater than two-fold change (absolute value of $\log 2$ (fold change) or $|\log 2 \mathrm{fc}|>1$ ) between strains and/or copper treatments (Table 2-S1) by all the four calculation methods for fold change.

\section{Quantitative Trait Loci Studies on Copper Response}

Strains from the recombinant haploid segregant collection ${ }^{17}$ from crossing S288c (S96) and YJM789 were grown to saturation in YM supplemented with lysine. Cells were then diluted to 0.2 $\mathrm{OD}_{600}$ in $200 \mu 1$ in $\mathrm{YM}$ or $\mathrm{YM}$ with $50 \mu \mathrm{M} \mathrm{CuSO}_{4}$ in triplicate in 96 well plates. Plates were sealed and readings taken every hour in a TECAN M200, an automatic plate reader with no shaking to mimic growth on solid media. Culture density at 12-hours (late-log phase) and at 18-hours (endpoint or stationary phase) were used to map quantitative trait loci (QTL). All readings in $\mathrm{Cu}$ were subtracted from the same strain grown in YM for QTL values (Table 2-S3). For each locus, we calculated a growth score and a significance score. To calculate the growth score, all strains that inherited the segregant YJM789 (1) allele were assigned to group 1, and all strains that inherited the segregant S96 (2) allele were assigned to group 2. Average growth was calculated for strains in group 1 and was subtracted from the average growth for strains in group 2 . The calculation resulted in the growth score. A positive growth score indicates that the segregant 1 allele leads to high growth and $\mathrm{Cu}$ resistance while a negative growth score indicates the segregant 2 allele confers $\mathrm{Cu}$ resistance. The significance score was also calculated, which is the probability of these growth scores occurring if the alleles were assigned randomly. To calculate the significance score, each yeast strain was randomly assigned to group 1 or group 2 regardless of that allele was inherited. The growth score was calculated given these 2 randomly assigned groups and repeated 100,000 times using a Monte Carlo simulation. The resulting distribution over growth scores was approximately normal. This normal distribution was used to calculate the significance score. We then studied the loci that have large magnitude growth scores and high significance scores for 12-hour growth (Table 2-S4) and 18-hour growth (Table 2-S5).

\section{Results}

\section{Clinical Isolates are More Sensitive to Copper than Agricultural Isolates}

$S$. cerevisiae is among the oldest domesticated species and has evolved high tolerances to copper independently multiple times both in the wild and in lab-evolution experiments ${ }^{5,25-29}$. To assess antimicrobial potency of copper incorporated onto cellulosic nanoparticles, genetically diverse yeast were incubated in liquid culture with different concentrations of c-CuNP [16]. The agricultural isolates AWRI1631 ${ }^{20}, \mathrm{RM} 11^{21,30,31}$, and $\mathrm{S} 288 \mathrm{c}^{32,33}$ were resistant to $\mathrm{Cu}$ whereas YJM339 $9^{19}$ and YJM789 19,34, clinical isolates of yeast, had lower $\mathrm{Cu}$ tolerances (Fig 2-1A). The cCuNPs were diluted to a level that would inhibit growth of YJM789 to a similar degree as $400 \mu \mathrm{M}$ of soluble $\mathrm{Cu}$ when measured for growth in a plate reader with no shaking, mimicking their growth 
on solid media. Both 30 $\mu \mathrm{g} / \mathrm{ml} \quad$ (by $\mathrm{CMC}$ concentration) of c-CuNP and $400 \mu \mathrm{M}$ of soluble copper decreased growth of YJM789 by $90 \%$ compared to YM media. Similarly, both the soluble $\mathrm{Cu}$ and c-CuNP embedded into solid agar media inhibited the growth of yeast, with the agricultural isolates being more resistant than the clinical isolates (Fig 21B). However, growth patterns on agar suggested that c-CuNPs may be more toxic than soluble $\mathrm{Cu}$, with growth at $10 \mu \mathrm{g} / \mathrm{ml} \quad \mathrm{c}-\mathrm{CuNP}$ inhibiting growth to the same extent as $200 \mu \mathrm{M}$ $\mathrm{Cu}$ (Fig 2-1B).

Because copper can catalyze the generation of A.

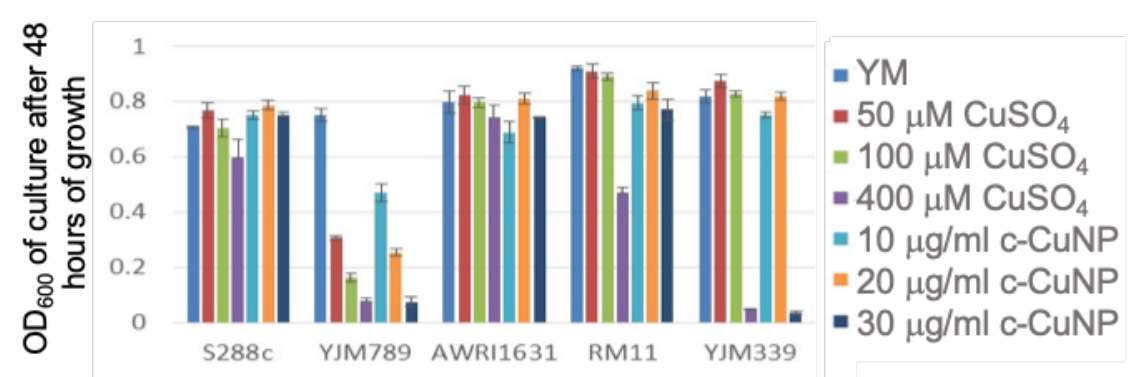

B.

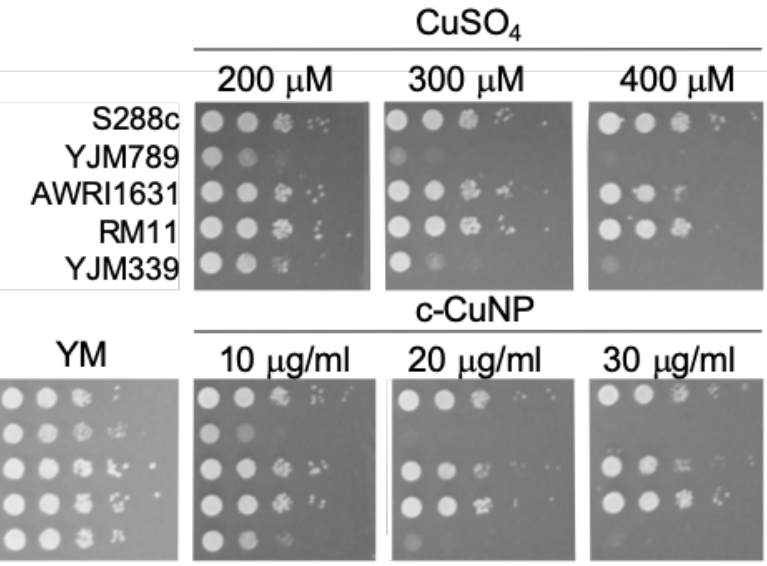

Figure 2-1. Impact of Genetic Variation in S. cerevisiae to Soluble Copper and Cellulosic Hybrid Copper Nanoparticles. A. Growth of yeast in liquid culture in synthetic media (YM) with an addition of dilutions of CuSO4 and c-CuNP. B. Serial dilutions of genetically diverse $S$. cerevisiae with dilutions of CuSO4 and c-CuNP on solid YM media.

reactive oxygen species ${ }^{2}$, we tested the ability of antioxidants to quench free radicals induced by soluble copper and c-CuNP. Both glutathione (GSH) and N-acetylcysteine (NAC), a glutathione precursor, rescued growth inhibition by soluble copper in clinical strains YJM789 and YJM339 (Fig 2-2). No growth rescue was detected with oxidized glutathione (GSSH) or ascorbic acid (another antioxidant) in the presence of $\mathrm{Cu}$ for YJM789, but there was a slight improvement for YJM339. When cells were treated with nicotinamide (NAM) and ascorbic acid (AA), the Cu tolerance of YJM339, but not YJM789, increased slightly, suggesting that, although YJM789 and YJM339 are $\mathrm{Cu}$ sensitive, there are other genetic differences that affect $\mathrm{Cu}$ response. However, NAC could not rescue growth inhibition caused by c-CuNPs. While NAC can function as an antioxidant when it is imported into cells, NAC is the precursor to glutathione, which is more potent at reducing $\operatorname{ROS}^{35}$. Therefore, while the pattern of c-CuNP sensitivity approximately mirrored soluble $\mathrm{Cu}$ sensitivity, the difference in NAC rescue suggests differences in Cu delivery, and likely, cellular internalization, between cells exposed to soluble $\mathrm{Cu}$ and c-CuNPs.

To determine how much $\mathrm{Cu}$ is imported into yeast, internal copper levels were measured by atomic absorption spectrometry (AAS). When grown in YM, YJM789 has three-fold less $\mathrm{Cu}$ than YJM339 or S288c. Furthermore, YJM789 accumulates as much $\mathrm{Cu}$ as RM11, a Cu-resistant strain (Fig 2-3). Thus, basal untreated internal $\mathrm{Cu}$ levels do not correspond to $\mathrm{Cu}$ sensitivity. Treatment with GSH or NAC did not significantly change internal copper levels except for in YJM789, in 
which cellular $\mathrm{Cu}$ increased with NAC to levels comparable to S288c and YJM339 (Fig 2-3). Treatment with $400 \mu \mathrm{M} \mathrm{Cu}$ increased internal $\mathrm{Cu}$ concentrations in all strains, although to varying extent, by between 9- and 90-fold (Fig 2-3). YJM789 and YJM339 had $\sim 0.8 \mu \mathrm{g}$ of internal Cu per $\mathrm{mg}$ of protein, whereas Cu-resistant strains S288c and RM11 had levels $66 \%$ to 53\% lower, respectively. To establish internal similar levels of copper to levels from c-CuNP as $400 \mu \mathrm{M}$ soluble copper, the amount of $\mathrm{c}-\mathrm{CuNP}$ was decreased to $157 \mu \mathrm{M}$ of $\mathrm{Cu}$ bound to $\mathrm{CMC}(=10 \mu \mathrm{g} / \mathrm{ml}$ $\mathrm{CMC}$ ). The acute exposure of yeast to $157 \mu \mathrm{M} \mathrm{Cu}$ as c-CuNP led to higher $\mathrm{Cu}$ accumulation than levels measured when exposed to soluble $\mathrm{Cu}$ (Fig 2-3): internal $\mathrm{Cu}$ concentrations of yeast treated with $157 \mu \mathrm{M} \mathrm{Cu}$ as c-CuNP were similar to yeast treated with 2.5-times higher levels of $\mathrm{CuSO}_{4}$. Co-treatment of yeast with glutathione and soluble $\mathrm{Cu}$ or $\mathrm{c}-\mathrm{CuNP}$ increased $\mathrm{Cu}$ uptake by 4 - to 10fold than copper treatments alone. The internal levels of $\mathrm{Cu}$ for $\mathrm{GSH}$ - and NAC-treated yeast, in general, were elevated and similar irrespective of copper source with two exceptions. YJM798 accumulated elevated $\mathrm{Cu}$ from c-CuNPs when supplemented with GSH and RM11 exposed to c$\mathrm{CuNP}$ and NAC had less internal $\mathrm{Cu}$ levels.

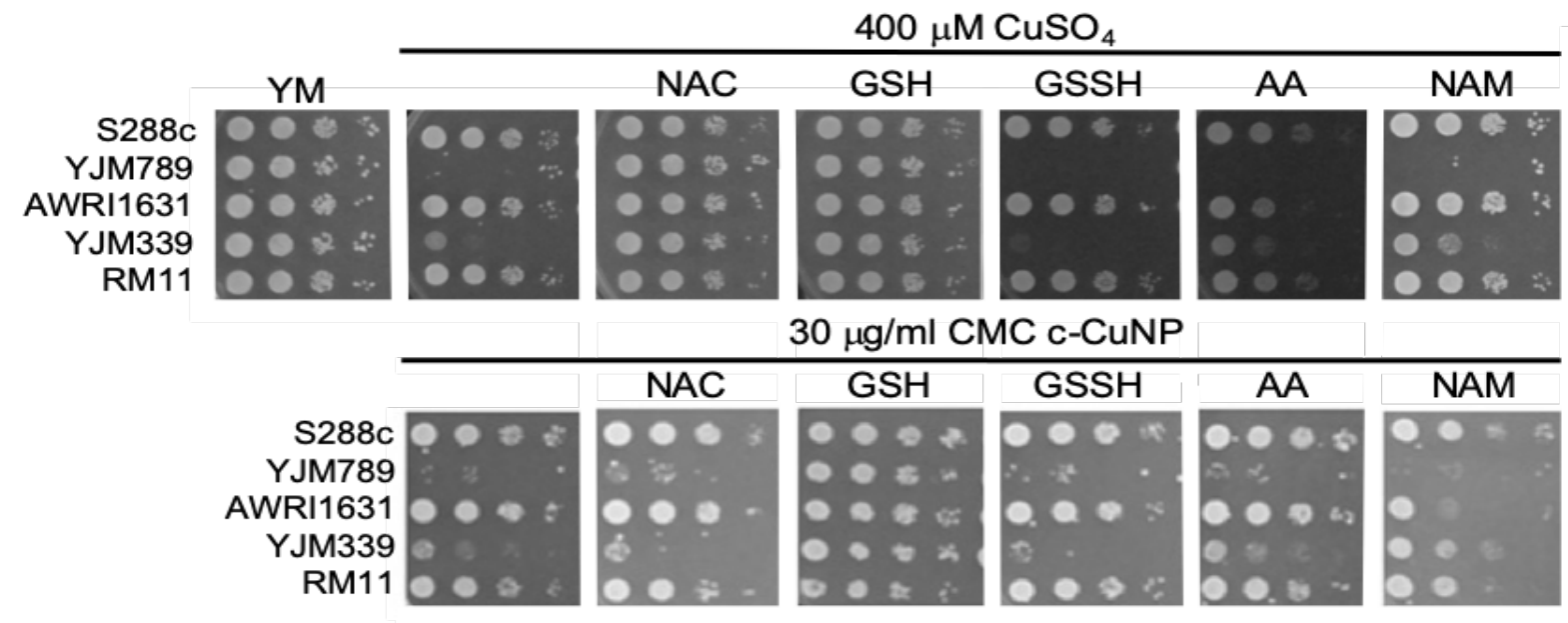

Figure 2-2 Rescue of Copper Nanoparticle Sensitivity with Antioxidants. Serial dilutions of genetically diverse $S$. cerevisiae on synthetic media (YM) with an addition of $400 \mu \mathrm{M} \mathrm{CuSO} 4$ or c-CuNPs and supplemented with $100 \mu \mathrm{M}$ of antioxidants as listed N-acetylcysteine (NAC), glutathione (GSH), oxidized glutathione (GSSH), ascorbic acid (AA) and nicotinamide (NAM). Haploid strains were serially diluted and spotted onto solid media. After 2 days of growth at $30^{\circ} \mathrm{C}$ plates were photographed.

Because the c-CuNPs increased efficiency of $\mathrm{Cu}$ delivery and lacked the NAC rescue to c-CuNP toxicity, we turned our focus to additional paths of $\mathrm{Cu}$ delivery by c-CuNP. c-CuNP are 10-20 nm particles with a unique three-dimensional spherical structure built on a cellulose scaffold ${ }^{16}$. To assess the interaction of c-CuNP with the outer surface of yeast cells, yeast cells were measured by flow cytometry in presence of c-CuNP. Yeast were stained with Rhodamine B, which crosses cell membranes and is sequestered by mitochondria without inducing cell lysis, while c-CuNPs were stained with FITC. Because c-CuNPs labeled with FITC are too small to be directly detected in flow cytometry, FITC labeling can only be measured when c-CuNPs are associated with yeast cells (Fig 2-S1). The fluorescence intensities of S288c, YJM789, YJM339, and RM11 cells incubated for two hours with $157 \mu \mathrm{M}$ of FITC pre-stained c-CuNPs increased compared to yeast cells alone (Fig 2-4A), suggesting the binding or uptake of c-CuNPs by yeast cells. Additionally, 
a greater proportion of cells of YLM789 and YJM339 accumulated FITC-labeled c-CuNPs (Fig 2-4A) and accumulated these to a greater extent (Fig 2-4B) than S288 c or RM11 lines.

To evaluate the physical location of c-CuNPs on yeast cells, cells exposed to FITC-labeled cCuNPs were further treated with trypan blue, which quenches FITC fluorescence. Trypan blue does not enter living cells and can only quench FITC if the dye was physically located outside the yeast. This addition decreased fluorescence intensity FITC-stained c-CuNP associated with yeast (Fig 2-4B). To confirm that c-CuNPs were both outside and inside, the yeast were again treated with c-CuNP and CMC (cellulose backbone of the nanoparticles). Further examination revealed two populations of cells: most of the FITC stained c-CuNPs appeared as speckled spots on the outside of the yeast, while about $10 \%$ of the cells exhibited fluorescence inside the cells as seen through z-stack using confocal microscopy (Fig 2-4C). The extracellular and intracellular fluorescence was not dependent on the presence of $\mathrm{Cu}$ on $\mathrm{CMC}$ (Fig 2-4C).

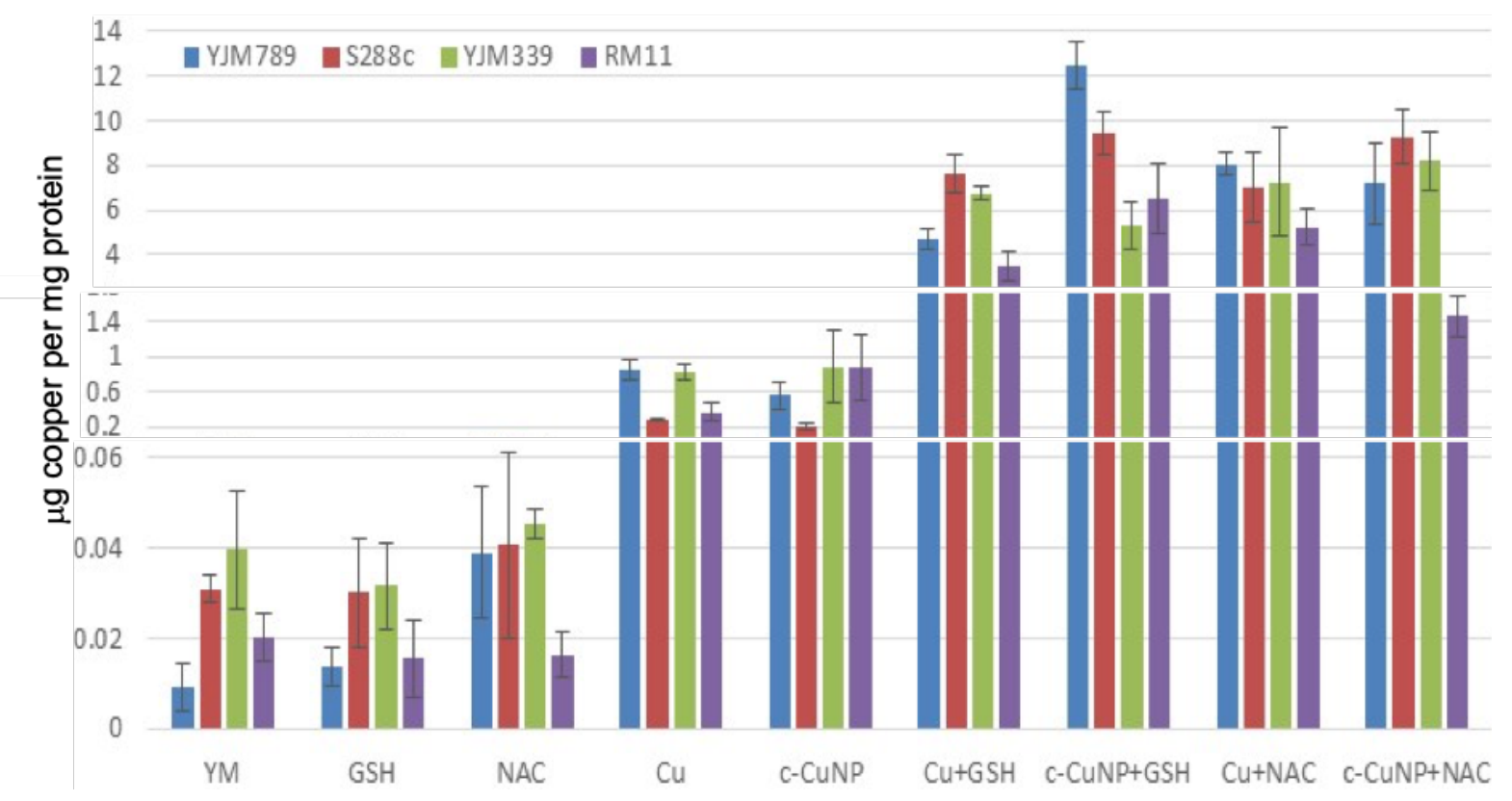

Figure 2-3. Internal Levels of Copper in Yeast Treated with Different Sources of Copper and Antioxidants. Internal levels of copper were measured by atomic absorption spectrometry and normalized to protein content. The following treatments are displayed in this graph: YM, $157 \mu \mathrm{M}$ c-CuNP, $400 \mu \mathrm{M} \mathrm{CuSO}, 100 \mu \mathrm{M} \mathrm{GSH}, 100 \mu \mathrm{M}$ NAC, $157 \mu \mathrm{M}$ c-CuNP + $100 \mu \mathrm{M}$ GSH, $157 \mu \mathrm{M}$ c-CuNP + $100 \mu \mathrm{M}$ NAC, $400 \mu \mathrm{M} \mathrm{CuSO}_{4}+100 \mu \mathrm{M}$ GSH, and $400 \mu \mathrm{M} \mathrm{CuSO}_{4}+100 \mu \mathrm{M}$ NAC. The graph is rescaled to better show the concentrations of the controls. The average of three independent samples for each treatment was determined and plotted error bars. 
To determine whether c-CuNPs were actively internalized by endocytosis, the viability of mutants in aly1 and aly2, arrestins that act as adapters for clatherinmediated endocytosis ${ }^{36}$, were evaluated. Also tested were mutants of frel and fre $2^{37,38}$, which reduce oxidized $\mathrm{Cu}$ for transport by $\mathrm{Ctr1}$. These knockout strains were constructed in the BY4741 strain background that is related to $\mathrm{S} 288 \mathrm{c}^{23}$. Testing the viability of yeast under acute exposure can reveal smaller differences in sensitivity and determine if changes in viability to c-CuNP liquid culture is different than on plates. Cells were grown in similar conditions as when measuring internal copper levels (Fig 2-3). When endocytosis is affected by deletion of Aly1 and Aly2, cellular viability was restored in the presence of c-CuNP but not soluble copper (Fig 2-4D). Loss of Fre1 and Fre2 increased cellular viability in the presence of soluble copper but not c-CuNP (Fig 24D).

Previous assessment of growth inhibition with c-CuNP was done under chronic 2day exposure measuring changes of growth on solid media (e.g., Figs 2-1 and 2-2). Physical interactions between yeast and c-CuNP were measured under acute exposure in liquid media (e.g., Figs 2-3 and 2-4). Viability assays were undertaken to evaluate the impact of soluble $\mathrm{Cu}$ and $\mathrm{c}$ CuNPs on cell survival under acute exposures at comparable total $\mathrm{Cu}$ concentrations. Overall, the greater toxicity of c-CuNPs is evident across all isolates, with near complete loss of viability following exposure to $400 \mu \mathrm{M} \mathrm{Cu}$ as c-CuNP (Fig 2-5). Differential resistance of the isolates was also confirmed, with S288c and RM11 exhibiting greater viability than YJM789

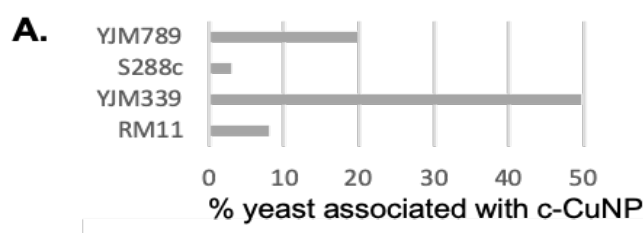

B.
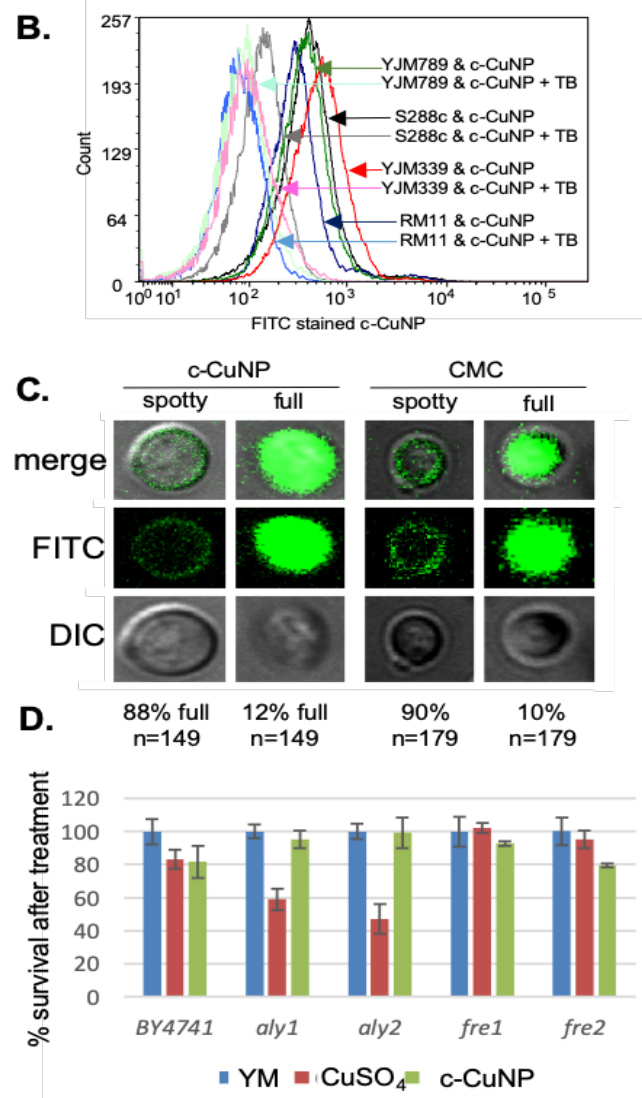

Figure 2-4. Physical Interaction of Copper Nanoparticle and Yeast Cells A. Flow cytometry measuring interaction between FITC stained c-CuNP and Rhodamine B stained yeast strains, YJM789 (YJM789K5a), S288c (GSY147), RM11 and YJM339. $157 \mu$ M. Dual stained c-CuNP and yeast are graphed as percentage. Yeast were stained with Rhodamine B. c-CuNP and yeast were then incubated for two hours. B. Flow cytometry of FITC stained c-CuNP associated with yeast were quenched with trypan blue (TB). Arrows point to peaks as they shift in fluorescence. The number of cells measured is on the $\mathrm{x}$-axis as count. Light lines represent yeast with c-CuNP and solid lines represent yeast and c-CuNP treated with trypan blue. C. Confocal microscopy of yeast (GSY147) and FITC stained c-CuNP. D. Cellular viability of yeast knockouts in the BY4741 (S288c) background normalized to yeast grown in YM supplemented with required amino acids treated with 400 $\mu \mathrm{M} \mathrm{CuSO}_{4}$ or $157 \mu \mathrm{M}$ c-CuNP. 
or YJM339 regardless of $\mathrm{Cu}$ source (Fig 2-5).

\section{Genetic and Proteomic Responses to Copper in Genetically Diverse Yeast}

The four diverse strains of yeast used in this study have underlying genetic differences that contribute to variation in $\mathrm{Cu}$ response. S288c and YJM789 exhibited the most extreme differences in their abilities to tolerate high doses of $\mathrm{Cu}$ and moderate doses of c-CuNP (Figs 2-1 and 1-2). To evaluate potential molecular underpinnings of these responses to $\mathrm{Cu}$, proteome- and genome-wide measurements were carried out. While many mRNAs are known to change in response to copper $^{7}$, we found only 22 proteins that showed at least 2-fold changes in S288c in response to $800 \mu \mathrm{M}$ copper

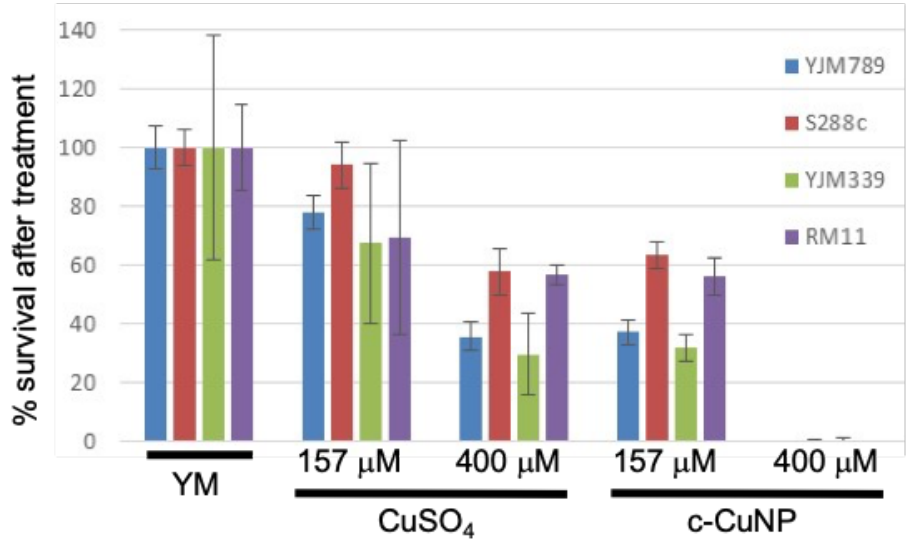

Figure 2-5. Acute Exposure of Yeast to Copper Nanoparticles. The number yeast forming colonies grown from cultures grown YM were normalized to $100 \%$. The viability after yeast after two hours of exposure to or $400 \mu \mathrm{M}$ and 157 $\mu \mathrm{M}$ of copper in the form of $\mathrm{CuSO}_{4}$ and c-CuNP is shown as a percentage compared to YM. by quantitative mass spectrometry (Table 2-1 and Table 2-S1). Many other proteins showed more than 2-fold changes between strains but not between copper treatments (Fig 2-S2). 19 out of the 22 copper-responsive proteins showed very little change (less than 1.2-fold change) in Cu-sensitive YJM789 cells when treated with 800 $\mu \mathrm{M} \mathrm{CuSO}_{4}$. Among the 19 proteins, Cup1, Fit2, Fet3, and Zrt1 were upregulated, all of which are involved in metal transport (Table 2-1). Cup1 is a protein that binds $\mathrm{Cu}$ intracellularly and mediates tolerance to high $\mathrm{Cu}$ concentrations ${ }^{39}$. The $\mathrm{S} 288 \mathrm{c}$ genome has two CUP1 alleles annotated with two synonymous SNPs and identical protein sequences (Cup1-1 and Cup1-2), and they cannot be differentiated in proteomics ${ }^{27}$. Cup1 ${ }^{\mathrm{YJM} 789}$ exhibited a higher abundance in YM relative to $\mathrm{Cup}^{\mathrm{S} 288 \mathrm{c}}$. Following $\mathrm{Cu}$ exposure, $\mathrm{Cup}^{\mathrm{YJM} 789}$ demonstrated lower abundance relative to $\mathrm{Cup}^{\mathrm{S} 288}$ due to the upregulation of Cup ${ }^{\mathrm{S} 288 \mathrm{c}}$ combined with little change in Cup $1^{\mathrm{YJM} 789}$ abundance (Table 2-1). Fit2 and Fet $3^{40,41}$ are involved in iron (Fe) transport, and $\mathrm{Zrt} 1$ is involved in $\mathrm{Zn}$ transport ${ }^{42}$. Fit2 and Zrt1 exhibited similar protein abundance between S288c and YJM789 in YM. After Cu exposure, Fit2 and Zrt1 showed higher abundance in S288c than in YJM789 because they were upregulated in S288c and changed little in YJM789 (Table 2-1). As for Fet3, S288c exhibited higher abundance than YJM789 in YM, and the upregulation by S288c in response to $\mathrm{Cu}$ exposure made strain-specific differences even greater (Table 2-2).

To further search for genetic loci contributing to $\mathrm{Cu}$ tolerance, we performed a genome-wide linkage study using a hybrid segregant collection ${ }^{17,43,44}$ to find association of genetic variation with differences in $\mathrm{Cu}$ tolerance between S288c and YJM789. 125 segregants from a S288c/YJM789 cross were grown at sub-lethal concentrations of copper $(50 \mu \mathrm{M})$ in liquid culture (Fig 2-S3 A-C). In contrast to previous experiments that used an acute exposure and high $\mathrm{Cu}$ concentration, we used a long exposure and lower dose of $\mathrm{Cu}$ to model the adaptive response to low level copper exposure. S288c grew better in low doses of $\mathrm{Cu}$ than in media with no additional $\mathrm{Cu}$ (Fig 2-S3C) 
as opposed to YJM789, which was strongly inhibited by $\mathrm{Cu}$ (Fig 2-S3B). In the presence of bathocuproinedisulfonic acid (BCS), a Cu chelator, S288c growth decreased (Fig 2-S3D). The differences in growth of haploid segregants between YM and $\mathrm{Cu}$ treatment at 12-hours (mid-log phase) and 18-hours (endpoint) were used as quantitative trait loci (QTL). Using a threshold of LOD (logarithm of odds) $>5$, three regions of the genome were identified at the mid-log phase (Table 2-S4 and Fig 2-S4A) and one region was identified at the 18-hour endpoint linked to $\mathrm{Cu}$ resistance (Table 2-S5 and Fig 2-S4B). The genetic variations within these regions are most likely associated with differences in copper tolerance between S288c and YJM789.

One region of the genome that was identified in the 12-hour, but not 18-hour, QTL mapping was

Table 2-1. Examples of Proteins Differentially Expressed (Log2-Fold Ratio) Between S288c and YJM789 with and without $800 \mu \mathrm{M} \mathrm{CuSO}$.

\begin{tabular}{|c|c|c|c|c|c|c|}
\hline $\begin{array}{c}\text { Order in } \\
\text { dendro- } \\
\text { gram }\end{array}$ & $\begin{array}{c}\text { Systematic } \\
\text { name }\end{array}$ & $\begin{array}{c}\text { Protein } \\
\text { name }\end{array}$ & $\begin{array}{c}\text { S288cCu/ } \\
\text { S288cYM }\end{array}$ & $\begin{array}{c}\text { YJM789Cu/ } \\
\text { YJM789YM }\end{array}$ & $\begin{array}{c}\text { YJM789YM/ } \\
\text { S288cYM }\end{array}$ & $\begin{array}{c}\text { YJM789Cu/ } \\
\text { S288cCu }\end{array}$ \\
\hline 81 & YHR053C/ & Cup1 & 3.477 & 0.196 & 2.585 & -0.686 \\
\hline & YHR055C & & & & & \\
\hline 82 & YOR382W & Fit2 & 2.448 & -0.117 & 0.167 & -2.387 \\
\hline 84 & YMR058W & Fet3 & 1.180 & -0.105 & -1.429 & -2.630 \\
\hline 93 & YGL255W & Zrt1 & 1.141 & 0.123 & -0.268 & -0.848 \\
\hline 63 & YDR424C & Dyn2 & -1.325 & -0.013 & 0.207 & 1.527 \\
\hline 64 & YAL007C & Erp2 & -1.119 & 0.228 & 0.386 & 1.743 \\
\hline 53 & YHR051W & Cox6 & -1.097 & -0.038 & 1.094 & 2.162 \\
\hline 60 & YOL077W-A & Atp19 & -1.327 & -0.195 & 1.525 & 2.667 \\
\hline 68 & YER057C & Hmf1 & -1.528 & 0.033 & 0.596 & 2.166 \\
\hline 65 & YIL051C & Mmf1 & -1.328 & -0.043 & 0.445 & 1.739 \\
\hline 58 & YBR230C & Om14 & -1.020 & -0.051 & 0.618 & 1.597 \\
\hline 71 & YOR130C & Ort1 & -1.236 & -0.050 & 0.010 & 1.205 \\
\hline 52 & YNL227C & Jjj1 & -1.050 & 0.015 & 0.796 & 1.869 \\
\hline 72 & YDL081C & Rpp1a & -2.002 & -0.009 & -0.373 & 1.629 \\
\hline 70 & YOR236W & Dfr1 & -1.134 & 0.574 & -0.346 & 1.429 \\
\hline 80 & YNL190W & Ynl190w & 1.474 & -0.756 & 3.492 & 1.271 \\
\hline 85 & YPR124W & Crt1 & -1.966 & -0.494 & -1.380 & 0.101 \\
\hline 35 & YAL046C & Aim1 & -0.168 & -1.334 & 1.136 & -0.021 \\
\hline 96 & YMR173W & Ddr48 & 0.653 & 0.006 & -0.564 & -1.202 \\
\hline
\end{tabular}

on chromosome VIII between coordinates 190,664 and 223,431, which contains twenty-two genes (Fig. 2-S4C). In the center of this region is the CUPl locus, and its contribution to Cu tolerance has been well studied. The common laboratory strain S288c and many other Cu-tolerant strains 
contain independent amplifications of the CUPI $\operatorname{locus}^{27,39}$. Other studies have also found that laboratory-evolved strains amplify this region of the genome $^{45}$. The Saccharomyces Genome Database reference strain, S288c, has two alleles of CUP1 with YHR054c, a gene of unknown function, between them. This region is actually amplified seven times in S288c with fourteen copies of the CUP1 gene; YJM789 contains one copy of CUP1 in each repetitive unit that is amplified seven times ${ }^{27}$.

To pinpoint the specific genes that were contributing to $\mathrm{Cu}$ tolerance, we focused on the genes that had amino acid polymorphisms or copy number variation. The region on chromosome $\mathrm{XI}$ between 74,608 and 92,505 (Fig 2-S4D) contained ten genes, among which MNN4, PEX1, MIA40, MST1, and $D H P 2$ encoded amino acid polymorphisms between
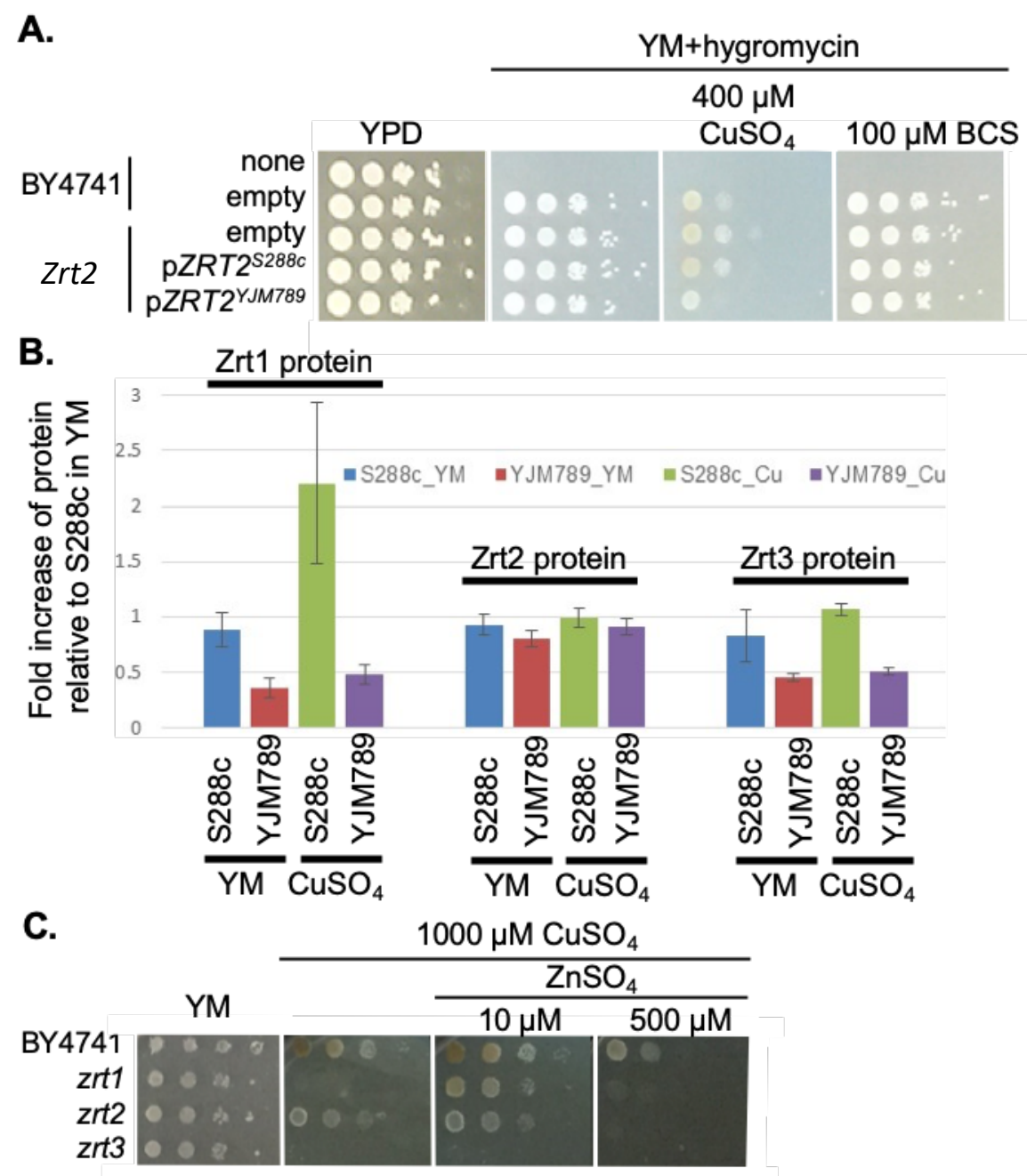

Figure 2-6. Impact of Zinc Transporters and Zinc on Copper Response. A. Zrt2 allele swaps in S288c (BY4741) in the presence of copper or BCS. ZRT2 plasmids were transformed and maintained in zrt2 mutants by the addition of hygromycin and strains were supplemented with necessary nutrients. B. Relative protein levels of three different zinc transporters in copper treated cells as measured from proteomic analysis. C. Serial dilution of S288c (BY4741) yeast with each of the zinc transporters deleted on increasing amounts of copper and zinc. After 2 days of growth at $30^{\circ} \mathrm{C}$ plates were photographed.

S288c and YJM789. Mia40 works with other proteins for the maturation of the mitochondrial fraction of Sod $1^{46}$. Sod1 is a major contributor to converting superoxides to hydrogen peroxide, which is further catalyzed into water and hydrogen ${ }^{47}$ and is important for tolerance to metals, including copper ${ }^{48}$. In our proteomic analysis, Sod 1 levels were not significantly changed between strains or in response to $\mathrm{Cu}$.

Another region that was identified in the endpoint linkage analysis was on chromosome II between coordinates 466,289 and 482,822 (Fig 2-S4B). Within this interval is the LYS2 gene, which is mutated in YJM789 as an auxotrophic marker. As this peak was not seen at the 12-hour time point (Fig 2-S4A), this may be interpreted as Lys2 becoming required as lysine present in the media was 
depleted as cells grew to saturation. Therefore, LYS2 may be selected by growth conditions instead of by $\mathrm{Cu}$ treatment.

In both 12- and 18-hour association analyses, one strong peak was shared on chromosome XII between 393,415 and 414,994 (2-S6 Figs 2-S4 A and B), which contained eleven genes (Fig 2-S4 $\mathrm{E})$. One of the genes known to be relevant to $\mathrm{Cu}$ tolerance was $A C E 2$, which encodes the activating transcription factor of CUP1 expression ${ }^{49}$. However, ACE2 showed no significant change in mRNA abundance in $\mathrm{YM}^{50}$, no amino acid polymorphisms between S288c and YJM789, and minimal change in protein levels in response to $\mathrm{Cu}$ exposure. Near $A C E 2$ was another gene of interest, $Z R T 2$, which encodes a low affinity $\mathrm{Zn}$ transporter with five amino acid polymorphisms between S288c and YJM78933. Like Ace2, Zrt2 exhibited less than 2-fold changes in protein abundance between strains or treatments. However, we cannot rule out changes in posttranslational modifications of $\mathrm{Zrt} 2$ in response to $\mathrm{Cu}$ or the impact of the polymorphisms on transporter function. Therefore, we cloned ZRT2 from each strain, including the endogenous promoters and terminators, into plasmids. When the plasmids were expressed in BY4741 zrt2 deleted yeast, only yeast expressing Zrt2 ${ }^{\mathrm{YJM} 789}$ grew more slowly on media containing $400 \mu \mathrm{M} \mathrm{Cu}$ and showed no change in growth on BCS (Fig. 2-6A). The polymorphisms within Zrt2 protein apparently contribute to the $\mathrm{Cu}$-sensitive phenotype of YJM789.

In the proteomic analysis, one other metal-responsive protein, Zrt1, was upregulated in S288c, but not in YJM789, when treated with $\mathrm{Cu}$ (Table 2-1). Zrt1 is a high affinity zinc transporter that is induced in $\mathrm{Zn}$ depleted cells ${ }^{42}$. While $\mathrm{Zrt1}^{\text {S288c }}$ was upregulated

approximately 2 -fold by $\mathrm{Cu}$ treatment, other $\mathrm{Zn}$ transporters, including Zrt2 and Zrt3, an intracellular $\mathrm{Zn}$ transporter, did not exhibit significant changes in response to $\mathrm{Cu}$ in either strain (Fig. 2-6B). YJM789 displayed slightly lower Zrt3 expression

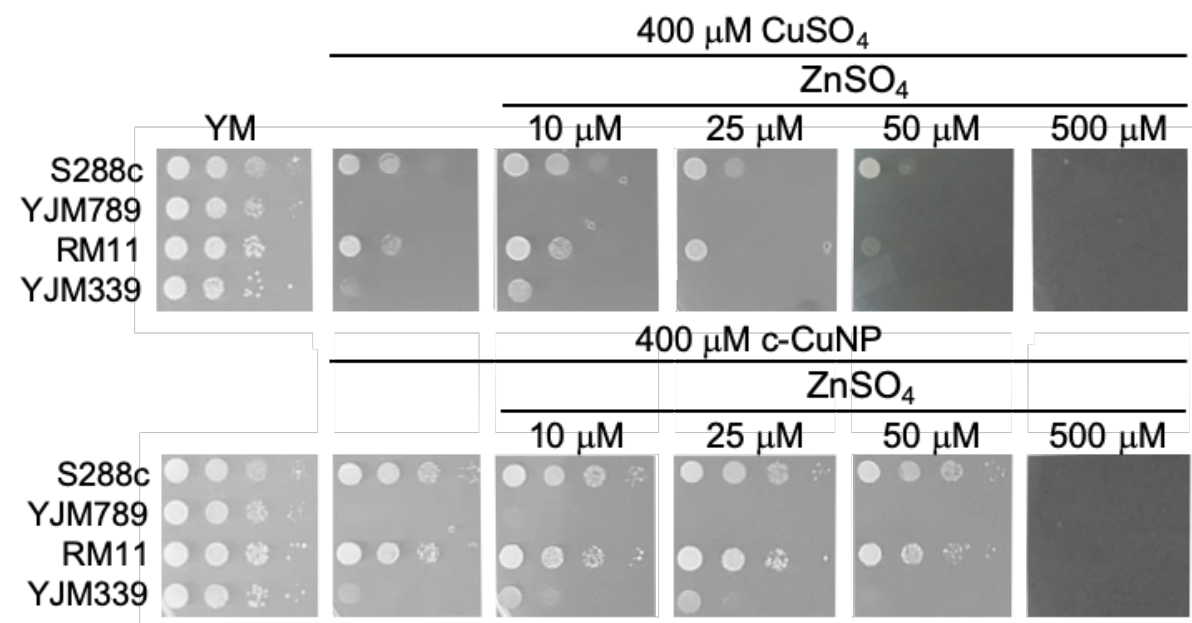

Figure 2-7. Addition of Zinc to Copper Nanoparticles Altered Growth Inhibition. Serial dilution of genetically diverse yeast (S288c (GSY147), YJM789 (YJM789K5a), AWRI1631, YJM339 and RM11 prototrophic strains) in response to copper and c-CuNP on increasing amounts of zinc sulfate. After 2 days of growth at $30^{\circ} \mathrm{C}$ plates were photographed.

compared to S288c

regardless of $\mathrm{Cu}$. Due to this association between $\mathrm{Zn}$ transporters and resistance to $\mathrm{Cu}$, we tested whether increased levels of exogenous $\mathrm{Zn}$ could the alter the $\mathrm{Cu}$ response of BY4741 and the $\mathrm{Zn}$ transporter mutant yeast lines. The zrt1 and zrt3 knockout lines were sensitive to $1000 \mu \mathrm{M} \mathrm{Cu}$, whereas the $z r t 2$ line was not (Fig 2-6C). The addition of $10 \mu \mathrm{M} \mathrm{Zn}$ improved the growth of $z r t 1$, but not $z r t 3$, mutants, and the $z r t 2$ line was still resistant to $\mathrm{Cu}$ and unaffected by supplemental $\mathrm{Zn}$ 
(Fig 2-6C). These growth promotion effects were lost at $500 \mu \mathrm{M} \mathrm{Zn}$ in the presence of $1000 \mu \mathrm{M}$ of $\mathrm{Cu}$ (Fig 2-6C).

To determine if the relationship between $\mathrm{Cu}$ and $\mathrm{Zn}$ was a more general yeast response, additional yeast strains were tested. When S288c, YJM339, and RM11 were grown in $400 \mu \mathrm{M}$ Cu in either the soluble form or from c-CuNP, adding $10 \mu \mathrm{M} \mathrm{Zn}$ resulted in modest growth increases, which were lost or became negative at and above $25 \mu \mathrm{M}$ Zn (Fig. 2-7). In contrast, YJM789 did not respond to supplemental Zn (Fig 2-7). These results indicate that Zn, at low concentrations, plays a role in modulating $\mathrm{Cu}$ toxicity and this benefit may result from different $\mathrm{Zn}$ uptake capacities among yeast strains.

\section{Discussion}

We have characterized the interaction of the genetically diverse model eukaryotic organism, $S$. cerevisiae, with soluble $\mathrm{Cu}$ and c-CuNPs towards improving future application of hybrid nanomaterials for microbial control. Copper has been used extensively over history as an antimicrobial agent, and microorganisms exposed to $\mathrm{Cu}$ gain the opportunity to develop resistance mechanisms to this metal. Metabolic mechanisms that reduce internal metal accumulation or free metal speciation, which will reduce the production of toxic ROS in the cytoplasm, are important in conferring heavy metal resistance. Overcoming existing microbial resistance pathways and altering the balance of metals in cells may enhance the efficacy of existing metal-based antimicrobial materials and aid in the development of novel antimicrobial agents.

\section{Cellulosic Copper Nanoparticles Exhibit Enhanced Toxicity}

In the current study, the antimicrobial potency of $\mathrm{Cu}$ was increased by the delivery of $\mathrm{Cu}$ in the form of c-CuNPs. Exposure of $157 \mu \mathrm{M}$ copper in NPs was as effective as $400 \mu \mathrm{M}$ copper sulfate in yeast growth inhibition, and increase in toxicity resulted from enhanced delivery of $\mathrm{Cu}$ to the cytoplasm (Figs 2-3 and 2-5). Flow cytometry evidence and c-CuNP fluorescence suggested that CuNPs bound in large part to the cell walls of $S$. cerevisiae and such binding may presage the transport of $\mathrm{Cu}$ into the cell (Fig 2-4B and C). Variation in binding of yeast cells and c-CuNPs mirrored resistance, with low binding strains exhibiting greater resistance to $\mathrm{Cu}$ (Fig 2-4A). This differential binding may enhance exposure in $\mathrm{Cu}$ sensitive strains. Based on the size of c-CuNPs $(10-20 \mathrm{~nm})^{16}$, it is also possible that c-CuNPs were absorbed into $S$. cerevisiae by endocytosis, and endocytotic activity varied between the strains. An endocytosis vesicle is $50-80 \mathrm{~nm}$ in $S$. cerevisiae $^{51}$ and some cells in the current study did exhibit internal fluorescence (Fig 2-4C). Although most of the yeast with incubated c-CuNPs appeared speckled with c-CuNP on the cell surface, approximately $10 \%$ of the yeast took up c-CuNPs as representing intracellular FITC staining. We were unable to test if these two subpopulation had differences in survival, but $13 \%$ of the laboratory yeast had internal FITC stained c-CuNP which was not statistically different from $18 \%$ of the yeast that fail to form colonies after c-CuNP treatment (Fig. 2-4C). Arrestin mutants in the laboratory strain (S288c background) displayed rescued cellular viability in the presence cCuNPs suggesting that endocytosis may be one mechanism of action of c-CuNPs, which would effectively deliver $\mathrm{Cu}$ to the cytoplasm. 


\section{Differential Expression of Metal Transporters is Linked to Toxicity}

Copper uptake and subsequent toxicity, regardless of the mechanism of enhanced delivery of copper via CuNPs, will most likely be governed by the reduction and transport of metals across the plasma membrane and their cellular compartmentation in different strains. In S288c, exposure to $\mathrm{Cu}$ down-regulated the $\mathrm{Cu}$ transporter, $\mathrm{Ctr}^{52}$, while expression of the $\mathrm{Cu}$ and $\mathrm{Fe}$ oxidizer, Fet $3^{53,54}$, the plasma membrane bound facilitator of iron transport, Fit3, and the high affinity Zn transporter, $\mathrm{Zrt1}$, increased. These patterns suggest that $\mathrm{Cu}$ exposure perturbs trace metal homeostasis and the more resistant S288c strain responds to these perturbations ${ }^{40,55}$. YJM789 did not show significant changes of these proteins and we propose that the lack of regulation of $\mathrm{Cu}$ uptake underlies the sensitivity of YJM789 to $\mathrm{Cu}$.

The expression levels of Crt1 tracked cell Cu concentrations in YJM789 and S288c. Expression is lower in YJM789 than S288c under control conditions, yet reverse under exposure to $\mathrm{Cu}$ (Table 2-1), leading to YJM789 exhibiting low basal $\mathrm{Cu}$ levels in YM medium, but elevated $\mathrm{Cu}$ under exposure to $\mathrm{Cu}$ (Fig 2-3). S288c regulation of expression more tightly regulates cell $\mathrm{Cu}$ concentrations in these conditions. Thus, S288c apparently has more responsive metabolic regulation of $\mathrm{Cu}$ uptake. Changes in other metal transport genes may reflect mass balance displacement of $\mathrm{Fe}$ and $\mathrm{Zn}$ by $\mathrm{Cu}$ from transporters or secondary stresses requiring additional $\mathrm{Fe}$ or $\mathrm{Zn}$ to overcome toxicity. Fet3 is a ferroxidase required for the reduction of $\mathrm{Fe}(\mathrm{II})$ and subsequent transport across the plasma membrane, and its upregulation may reflect increased demand for $\mathrm{Fe}$ due to inhibition of $\mathrm{Fe}(\mathrm{II})$ transport by $\mathrm{Cu}$ or increased demand for redox metabolism within the cell. Similarly, Fit2 is an iron and iron-siderophore-binding protein expressed on the cell surface ${ }^{40}$ that facilitates $\mathrm{Fe}$ uptake and may be directly regulating $\mathrm{Cu}$ response by adjusting internal iron levels for Sod2, a mitochondrial superoxide dismutase ${ }^{56}$.

The up-regulation of $\mathrm{Zrt} 1$, the high affinity $\mathrm{Zn}$ transporter, also points to $\mathrm{Cu}$-induced $\mathrm{Zn}$ limitation in $S$. cerevisiae. High affinity transporters are typically activated by mineral deficiency, and the low $k_{\mathrm{m}}$ for Zrt1 in yeast $(\sim 10 \mathrm{nM})$ suggests it would be effective in overcoming deficiency ${ }^{57}$. That supplemental Zn rescued zrtl knockouts (Fig 2-6) and, to a lesser degree, native strains under high $\mathrm{Cu}$ exposure (Fig 2-7) suggests that excess $\mathrm{Cu}$ ions at the plasma membrane surface may be impeding the binding of $\mathrm{Zn}$ to $\mathrm{Zn}$ transporters. It is interesting that the more $\mathrm{Cu}$ resistant strain S288c upregulates Zrt1 whereas the sensitive YJM789 does not, suggesting that metal homeostasis responses may be limited in cases where excessive $\mathrm{Cu}$ accumulation does not lead to loss of cellular signaling systems or excessive ROS damage as would occur in strains that cannot limit $\mathrm{Cu}$ accumulation (Fig 2-3).

\section{Intracellular Copper Binding Reduces Copper Toxicity}

Transporters, intracellular sequestration, and chaperonins/chelation maintain metal concentrations within strict bounds and tightly regulate the balance and potential toxicity of metals within the cytoplasm. In the current study, S288c up-regulated expression of Cup1, the main metallothionein in S. cerevisiae, in response to Cu. YJM789 did not show significant changes in levels of this metal-binding protein in the presence of $\mathrm{Cu}$ and we propose that the lack of response to increased cytoplasmic Cu increased YJM789 sensitivity to this metal. Unregulated free Cu concentrations in the cytoplasm will increase the production of ROS and lead to broad disruptions in cell homeostasis. 
In support of this, providing certain antioxidants increased the copper levels of all treated strains (Fig 2-2). Glutathione can bind copper transported into cells by Ctr1 before delivery to copper chaperones $^{58}$. More importantly, glutathione and NAC may serve to prime phytochelatin and metallothionein production within $S$. cerevisiae ${ }^{59,60}$. These peptides/proteins function to regulate internal free metal concentrations and maintain cytoplasmic metal homeostasis. The large increases in $\mathrm{Cu}$ accumulation from both soluble $\mathrm{Cu}$ and $\mathrm{CuNPs}$ may reflect enhanced production of these metal-binding systems, the chelation of $\mathrm{Cu}$ intercellularly, and internal storage of nontoxic metal complexes.

\section{Addition of Zinc Increases Copper Toxicity}

Although the toxicity of $\mathrm{Cu}$ was alleviated in some strains by the addition of low concentrations of $\mathrm{Zn}$ as discussed above, the antimicrobial activity of $\mathrm{Cu}$ was enhanced in the current study by the application of higher $\mathrm{Zn}$ concentrations (Fig 2-7). As with $\mathrm{Cu}, \mathrm{Zn}$ plays critical roles as a catalytic and structural cofactor in a wide array of proteins, and maintenance of free $\mathrm{Zn}^{2+}$ concentrations within the cytoplasm is important in maintaining metal homeostasis ${ }^{61}$. Zinc is transported in the cell by the membrane-bound transporters Zrt1 and Zrt2 and is sequestered in the vacuole by $\mathrm{Zrt} 3^{62}$. Given that exposure of yeast to $\mathrm{Cu}$ induces the expression of $\mathrm{Zrt} 1$ in $\mathrm{Cu}$-tolerant strains (Table 2-1), the addition of Zn may into the environment may lead to the over-accumulation of $\mathrm{Zn}$ and subsequent toxicity to $S$. cerevisiae.

When in excess, $\mathrm{Zn}$ will induce many of the same lesions in $S$. cerevisiae as does $\mathrm{Cu}$, including oxidative damage and negative interactions with $\mathrm{Fe}$ transport. Thus, the enhanced microbial activity of $\mathrm{Cu}$ and $\mathrm{Zn}$ combined may be due to the additional sympatric lesions induced by $\mathrm{Zn}$. However, there is evidence that the stresses induced by $\mathrm{Cu}$ and $\mathrm{Zn}$ are distinctly different. In a unique multi-metal study of $S$. cerevisiae response to stress, ${ }^{63,64}$ found significant differences between the response of yeast to $\mathrm{Cu}$ and $\mathrm{Zn}$. While there was some overlap in the common stress responses, such as metal transport and oxidative stress, significant groups of differentially expressed genes did not overlap and only two of the 10 most metal-responsive protein-protein and protein-DNA interacting networks were shared ${ }^{64}$. Thus, there may be limited overlap in the cell's metabolic rescue capacity when, under $\mathrm{Cu}$ exposure, a second divergent metal is introduced. The addition of $\mathrm{Zn}$ to the production of $\mathrm{Cu}$-based nanomaterials would improve the antimicrobial properties of these novel antimicrobial systems and may represent a new approach for public health and food safety.

\section{Acknowledgements}

We thank Mike Snyder for use of the Orbitrap used in these studies. Randall Mann generously shared his common protein contaminant list. Angela Lee generously gave us the yeast knockout collection. Jenay Grant assisted in confirming the genomic amplification of the CUP1 locus. Flow cytometry was carried out on the WVU Flow Cytometry \& Single Cell Core Fortessa. Microscopy was done with the aid of Phil Chapman.

\section{Author Contributions}

XRM carried out the proteomics analysis and LJ developed proteomics workflow. MJW carried out the microscopy, characterized Zrt2, and flow cytometry with JBL. JBL and VAP carried out viability assays. LMW and JRC measured internal copper concentrations. TWC analyzed QTL 
data. ZRL characterized Cup1 levels. ZRL, JRC, and JEGG wrote the manuscript. JEGG conceived and directed the experiments. 


\section{References}

1. Dollwet, H. H. A. \& Sorenson, J. R. J. Historic uses of copper compounds in medicine. in Trace elements in Medicine 2, 80-87 (1985).

2. Lloyd, R. V, Hanna, P. M. \& Mason, R. P. The origin of the hydroxyl radical oxygen in the Fenton reaction. Free Radic. Biol. Med. 22, 885-888 (1997).

3. Avery, S. V, Howlett, N. G. \& Radice, S. Copper toxicity towards Saccharomyces cerevisiae: dependence on plasma membrane fatty acid composition. Appl. Environ. Microbiol. 62, 3960-3966. (1996).

4. Quaranta, D. et al. Mechanisms of Contact-Mediated Killing of Yeast Cells on Dry Metallic Copper Surfaces. Appl. Environ. Microbiol. 77, 416-426 (2011).

5. Samanovic, M. I., Ding, C., Thiele, D. J. \& Darwin, K. H. Copper in microbial pathogenesis: Meddling with the metal. Cell Host Microbe 11, 106-115 (2012).

6. Lyons, T. J. et al. Genome-wide characterization of the Zap1p zinc-responsive regulon in yeast. Proc. Natl. Acad. Sci. U. S. A. 97, 7957-7962 (2000).

7. Gross, C., Kelleher, M., Iyer, V. R., Brown, P. O. \& Winge, D. R. Identification of the Copper Regulon in Saccharomyces cerevisiae by DNA Microarrays. Journal of Biological Chemistry 275, 32310-32316 (2000).

8. Wegner, T. \& Jones, P. Nanotechnology for the forest products industry. Wood Fiber Sci. 37, 549-551 (2005).

9. Wegner, T. H. \& Jones, P. H. Advancing cellulose-based nanotechnogy. Adv. Cellul. nanotechnology. Cellulose 13, 115-118 (2006).

10. Wegner, T. H. \& Jones, E. P. A fundamental review of the relationships between nanotechnology and lignocellulosic biomass. Nanosci. Technol. Renew. Biomater. 1, 1-41 (2009).

11. McCrank, J. Nanotechnology applications in the forest sector. (Natural Resources Canada, 2009).

12. Moon, R. J., Martini, A., Nairn, J., Simonsen, J. \& Youngblood, J. Cellulose nanomaterials review: structure, properties and nanocomposites. Chem. Soc. Rev. 40, 3941-3994 (2011).

13. Cai, J., Kimura, S., Wada, M. \& Kuga, S. Nanoporous cellulose as metal nanoparticles support. Biomacromolecules 10, 87-94 (2008).

14. Zhong, T., Oporto, G. S., Jaczynski, J. \& Jiang, C. Nanofibrillated Cellulose and Copper Nanoparticles Embedded in Polyvinyl Alcohol Films for Antimicrobial Applications. Biomed Res. Int. 2015, 1-8 (2015).

15. Jiang, C., Oporto, G. S., Zhong, T. \& Jaczynski, J. TEMPO nanofibrillated cellulose as template for controlled release of antimicrobial copper from PVA films. Cellulose 23, 713722 (2016).

16. Zhong, T., Oporto, G. S., Jaczynski, J., Tesfai, A. T. \& Armstrong, J. Antimicrobial properties of the hybrid copper nanoparticles-carboxymethyl cellulose. Wood Fiber Sci. 45, 215-222 (2013).

17. Steinmetz, L. M. et al. Dissecting the architecture of a quantitative trait locus in yeast. Nature 416, 326-330 (2002).

18. Wenger, J. W., Schwartz, K. \& Sherlock, G. Bulk segregant analysis by high-throughput sequencing reveals a novel xylose utilization gene from Saccharomyces cerevisiae. PLoS Genet. 6, 18 (2010).

19. McCusker, J. H., Clemons, K. V, Stevens, D. A. \& Davis, R. W. Genetic characterization 
of pathogenic Saccharomyces cerevisiae isolates. Genetics 136, 1261-1269 (1994).

20. Borneman, A. R., Forgan, A. H., Pretorius, I. S. \& Chambers, P. J. Comparative genome analysis of a Saccharomyces cerevisiae wine strain. FEMS Yeast Res 8, 1185-1195 (2008).

21. Mortimer, R. K., Romano, P., Suzzi, G. \& Polsinelli, M. Genome renewal: a new phenomenon revealed from a genetic study of 43 strains of Saccharomyces cerevisiae derived from natural fermentation of grape musts. Yeast 10, 1543-1552 (1994).

22. McCullough, M. J., Clemons, K. V, Farina, C., McCusker, J. H. \& Stevens, D. A. Epidemiological investigation of vaginal Saccharomyces cerevisiae isolates by a genotypic method. J Clin Microbiol 36, 557-562 (1998).

23. Tong, A. H. et al. Systematic genetic analysis with ordered arrays of yeast deletion mutants. Science (80-. ). 294, 2364-2368 (2001).

24. Wisniewski, J. R., Zougman, A. \& Mann, M. Combination of FASP and StageTip-based fractionation allows in-depth analysis of the hippocampal membrane proteome. J Proteome Res 8, 5674-5678 (2009).

25. Gerstein, A. C. et al. Too much of a good thing: the unique and repeated paths toward copper adaptation. Genetics 199, 555-71 (2015).

26. Hodgins-Davis, A., Adomas, A. B., Warringer, J. \& Townsend, J. P. Abundant gene-byenvironment interactions in gene expression reaction norms to copper within Saccharomyces cerevisiae. Genome Biol Evol 4, 1061-1079 (2012).

27. Zhao, Y. et al. Structures of naturally evolved CUP1 tandem arrays in yeast indicate that these arrays are generated by unequal nonhomologous recombination. G3 (Bethesda). 4, 2259-2269 (2014).

28. Fay, J. C., McCullough, H. L., Sniegowski, P. D. \& Eisen, M. B. Population genetic variation in gene expression is associated with phenotypic variation in Saccharomyces cerevisiae. Genome Biol 5, R26 (2004).

29. Kvitek, D. J., Will, J. L. \& Gasch, A. P. Variations in stress sensitivity and genomic expression in diverse S. cerevisiae isolates. PLoS Genet 4, e1000223 (2008).

30. Torok, T., Mortimer, R. K., Romano, P., Suzzi, G. \& Polsinelli, M. Quest for wine yeasts-an old story revisited. J. Ind. Microbiol. 17, 303-313 (1996).

31. Brem, R. B., Yvert, G., Clinton, R. \& Kruglyak, L. Genetic dissection of transcriptional regulation in budding yeast. Science (80-. ). 296, 752-755 (2002).

32. Mortimer, R. K. \& Johnston, J. R. Genealogy of principal strains of the yeast genetic stock center. Genetics 113, 35-43 (1986).

33. Cherry, J. M. et al. Saccharomyces Genome Database: the genomics resource of budding yeast. Nucleic Acids Res. 40, D700-5 (2012).

34. Tawfik, O. W., Papasian, C. J., Dixon, A. Y. \& Potter, L. M. Saccharomyces cerevisiae pneumonia in a patient with acquired immune deficiency syndrome. J Clin Microbiol 27, 1689-1691 (1989).

35. Sadowska, A. M. N-Acetylcysteine mucolysis in the management of chronic obstructive pulmonary disease. Ther. Adv. Respir. Dis. 6, 127-135 (2012).

36. Lin, C. H., MacGurn, J. A., Chu, T., Stefan, C. J. \& Emr, S. D. Arrestin-Related UbiquitinLigase Adaptors Regulate Endocytosis and Protein Turnover at the Cell Surface. Cell 135, 714-725 (2008).

37. Georgatsou, E., Mavrogiannis, L. A., Fragiadakis, G. S. \& Alexandraki, D. The yeast Fre1p/Fre2p cupric reductases facilitate copper uptake and are regulated by the coppermodulated Mac1p activator. J. Biol. Chem. 272, 13786-13792 (1997). 
38. Yamaguchi-Iwai, Y. et al. Homeostatic regulation of copper uptake in yeast via direct binding of Mac1 protein to upstream regulatory sequences of FRE1 and CTR1. J. Biol. Chem. 272, 17711-17718 (1997).

39. Karin, M. et al. Primary structure and transcription of an amplified genetic locus: The CUP1 locus of yeast. Proc. Natl. Acad. Sci. U. S. A. 81, 337-341 (1984).

40. Protchenko, O. et al. Three cell wall mannoproteins facilitate the uptake of iron in Saccharomyces cerevisiae. J. Biol. Chem. 276, 49244-49250 (2001).

41. Silva, D. M. De et al. The FET3 gene product required for high affinity iron transport in yeast is a cell surface ferroxidase. J. Biol. Chem. 270, 1098-1101 (1995).

42. Zhao, H. \& Eide, D. The yeast ZRT1 gene encodes the zinc transporter protein of a highaffinity uptake system induced by zinc limitation. Proc. Natl. Acad. Sci. 93, 2454-2458 (1996).

43. Gallagher, J. E. G. et al. Divergence in a master variator generates distinct phenotypes and transcriptional responses. Genes Dev 28, 409-421 (2014).

44. Mancera, E., Bourgon, R., Brozzi, A., Huber, W. \& Steinmetz, L. M. High-resolution mapping of meiotic crossovers and non-crossovers in yeast. Nature 454, 479-485 (2008).

45. Adamo, G. M., Lotti, M., Tamas, M. J. \& Brocca, S. Amplification of the CUP1 gene is associated with evolution of copper tolerance in Saccharomyces cerevisiae. Microbiology 158, 2325-2335 (2012).

46. Varabyova, A. et al. Mia40 and MINOS act in parallel with Ces1 in the biogenesis of mitochondrial Sod1. FEBS J. 280, 4943-4959 (2013).

47. Tamai, K. T., Gralla, E. B., Ellerby, L. M., Valentine, J. S. \& Thiele, D. J. Yeast and mammalian metallothioneins functionally substitute for yeast copper-zinc superoxide dismutase. Proc. Natl. Acad. Sci. 90, 8013-8017 (1993).

48. Pena, M. M., Koch, K. A. \& Thiele, D. J. Dynamic regulation of copper uptake and detoxification genes in Saccharomyces cerevisiae. Mol. Cell. Biol. 18, 2514-2523 (1998).

49. Butler, G. \& Thiele, D. J. ACE2, an activator of yeast metallothionein expression which is homologous to SWI5. Mol. Cell. Biol. 11, 476-485 (1991).

50. Zheng, W., Zhao, H., Mancera, E., Steinmetz, L. M. \& Snyder, M. Genetic analysis of variation in transcription factor binding in yeast. Nature 464, 1187-1191 (2010).

51. Buser, C. \& Drubin, D. G. Ultrastructural imaging of endocytic sites in Saccharomyces cerevisiae by transmission electron microscopy and immunolabeling. Microsc Microanal 19, 381-392 (2013).

52. Knight, S. A., Labbe, S., Kwon, L. F., Kosman, D. J. \& Thiele, D. J. A widespread transposable element masks expression of a yeast copper transport gene. Genes Dev. 10, 1917-1929 (1996).

53. de Silva, D., Davis-Kaplan, S., Fergestad, J. \& Kaplan, J. Purification and characterization of Fet3 protein, a yeast homologue of ceruloplasmin. J. Biol. Chem. 272, 14208-14213 (1997).

54. Askwith, C. et al. The FET3 gene of S. cerevisiae encodes a multicopper oxidase required for ferrous iron uptake. Cell 76, 403-410 (1994).

55. Stadler, J. A. \& Schweyen, R. J. The yeast iron regulon is induced upon cobalt stress and crucial for cobalt tolerance. J. Biol. Chem. 277, 39649-54 (2002).

56. Naranuntarat, A., Jensen, L. T., Pazicni, S., Penner-Hahn, J. E. \& Culotta, V. C. The interaction of mitochondrial iron with manganese superoxide dismutase. J. Biol. Chem. 284, 22633-22640 (2009). 
57. Eide, D. J. Zinc transporters and the cellular trafficking of zinc. Biochim. Biophys. Acta Mol. Cell Res. 1763, 711-722 (2006).

58. Maryon, E. B., Molloy, S. A. \& Kaplan, J. H. Cellular glutathione plays a key role in copper uptake mediated by human copper transporter 1. Am. J. Physiol. Physiol. 304, C768-79 (2013).

59. Kneer, R., Kutchan, T. M., Hochberger, A. \& Zenk, M. H. Saccharomyces cerevisiae and Neurospora crassa contain heavy metal sequestering phytochelatin. Arch. Microbiol. 157, 305-310 (1992).

60. Wünschmann, J. et al. Phytochelatins are synthesized by two vacuolar serine carboxypeptidases in Saccharomyces cerevisiae. FEBS Lett. 581, 1681-1687 (2007).

61. MacDiarmid, C. W., Gaither, L. A. \& Eide, D. Zinc transporters that regulate vacuolar zinc storage in Saccharomyces cerevisiae. EMBO J. 19, 2845-2855 (2000).

62. Winston, F., Dollard, C. \& Ricupero-Hovasse, S. L. Construction of a set of convenient Saccharomyces cerevisiae strains that are isogenic to S288C. Yeast 11, 53-55 (1995).

63. Liu, X. et al. Characterisation of two quorum sensing systems in the endophytic Serratia plymuthicastrain G3: differential control of motility and biofilm formation according to lifestyle. BMC Microbiol. 11, 26 (2011).

64. Jin, Y. H. et al. Global Transcriptome and Deletome Profiles of Yeast Exposed to Transition Metals. PLoS Genet. 4, e1000053 (2008). 


\section{Appendix A}

\section{Supplemental Figures}
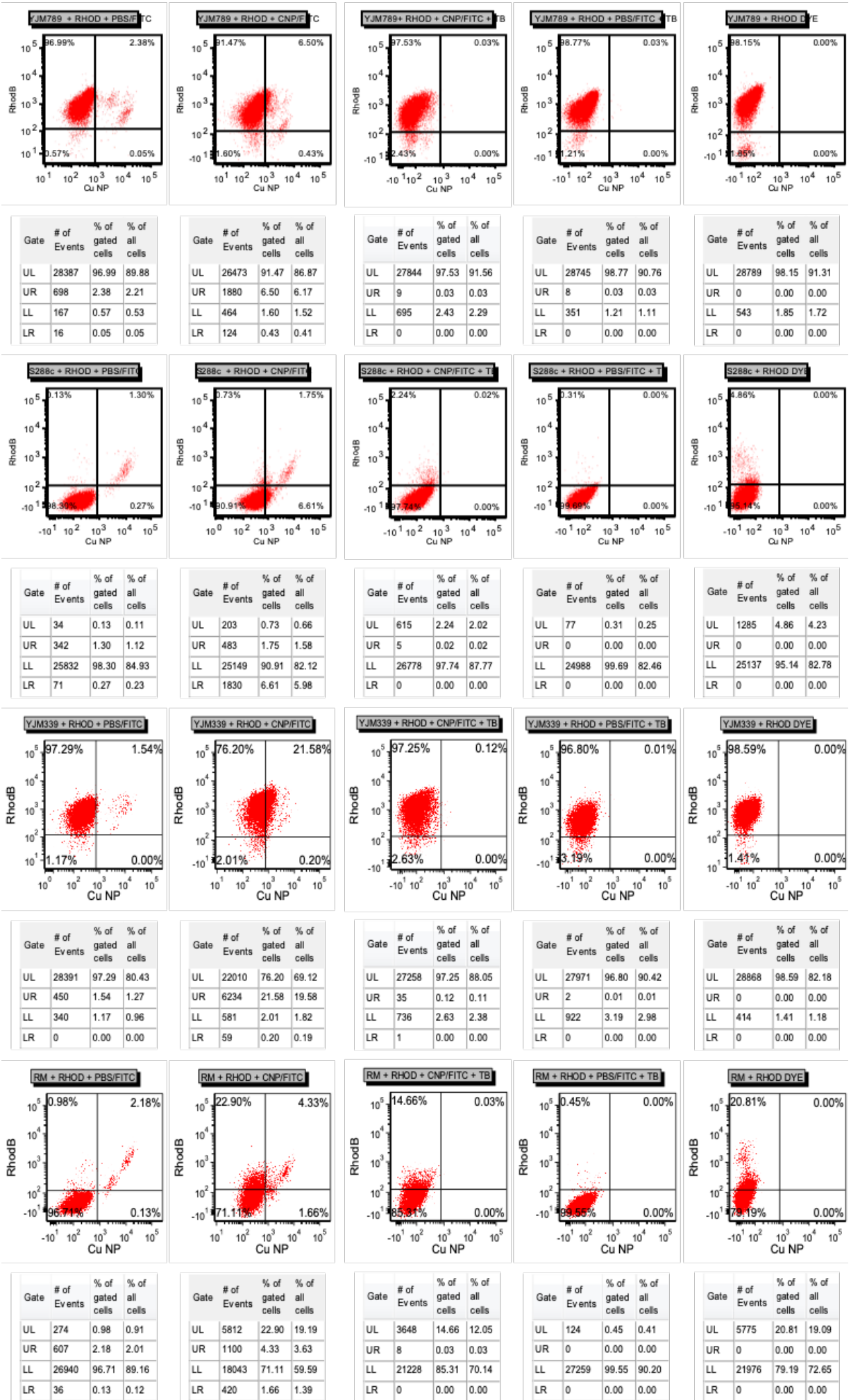

Figure 2- S1. Flow cytometry of individually stained yeast. 


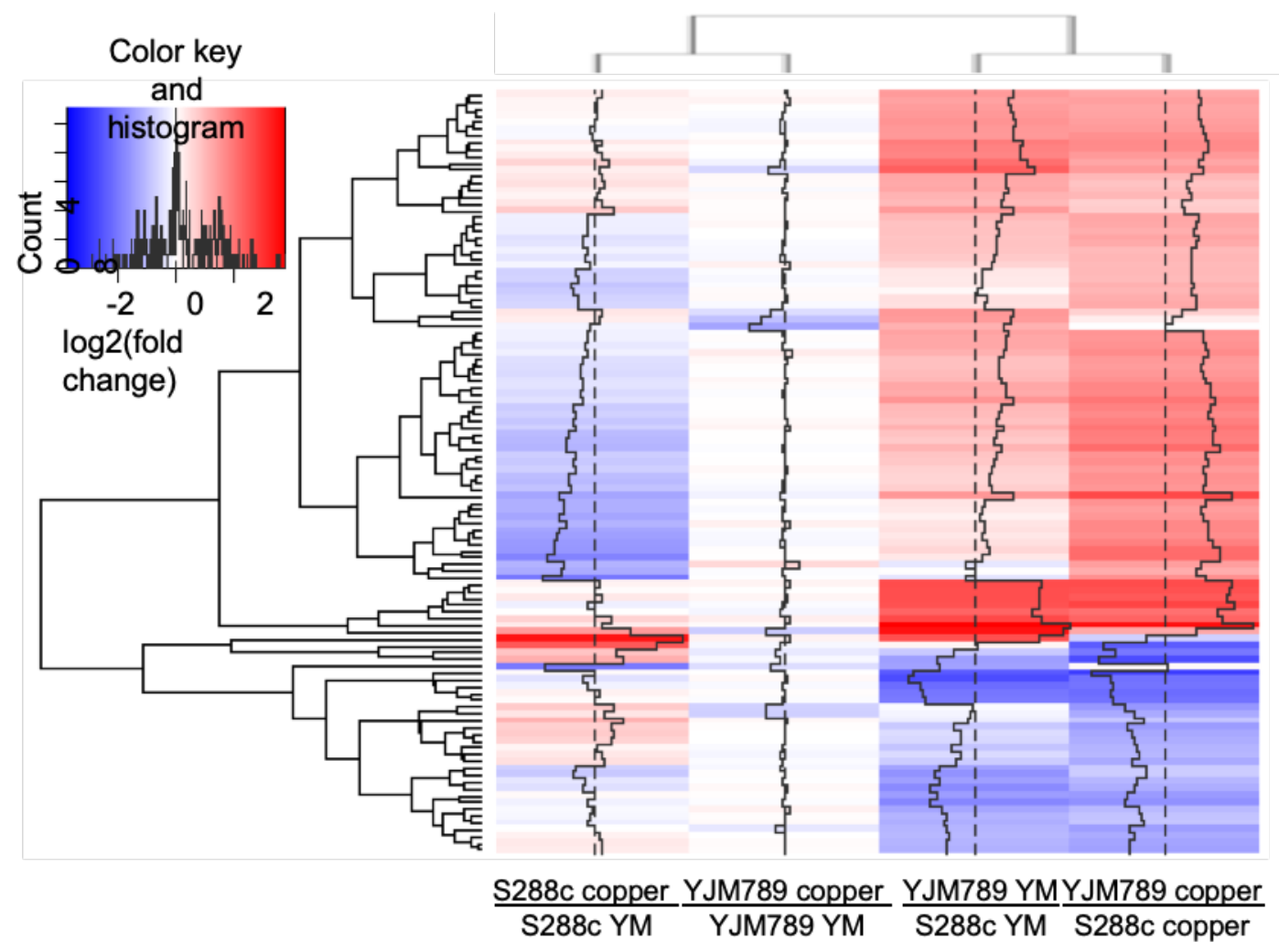

Figure 2- S2. Hierarchical Clustering of Protein Levels from Proteomic Analysis of Yeast Treated with Copper Reveals Inherent Characteristics of Yeast Strains. Protein levels from S288c (S96) and YJM789 treated with $800 \mu \mathrm{M}$ of $\mathrm{CuSO}_{4}$ for 90 minutes were clustered hierarchically. 
A.

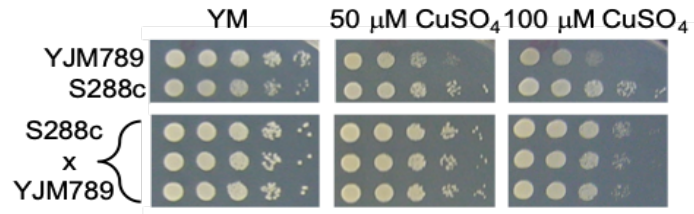

B.

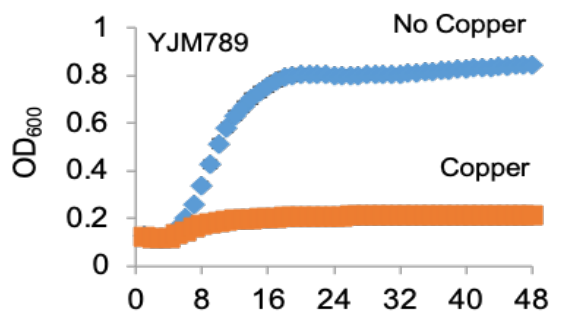

C.

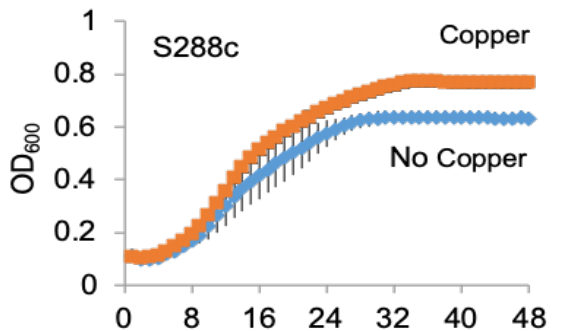

D.

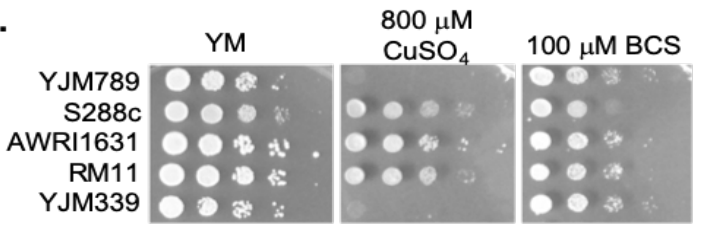

Figure 2- S3. Growth Assays of Genetically Diverse Yeast in Media with Excess and Limited Copper. A. Copper resistance was determined to be a dominant trait in hybrids generated from mating YJM789, a copper sensitive strain, with S288c (S96), a copper resistant strain. Growth patterns of the hybrids in the presence of excess copper were similar to S288c. B. and C. Example growth curves developed for the two parent strains grown in $\mathrm{YM}$ and $\mathrm{YM}$ with $50 \mu \mathrm{M} \mathrm{CuSO}_{4}$. Growth patterns were analyzed for 125 unique segregants developed; segregants displayed a wide variety of resistance, ranging from extremely sensitive to extremely resistant. D. Serial dilution of genetically diverse yeast strains (YJM789, S288c (GSY1470, AWRI1631, RM11, and YJM339) grown on YM plates $\begin{array}{llllllll}\text { containing } & 400 & \mu \mathrm{M} & \mathrm{CuSO}_{4} & \text { or } & 100 & \mu \mathrm{M}\end{array}$ bathocuproinedisulfonic acid (BCS), a copper chelator. 


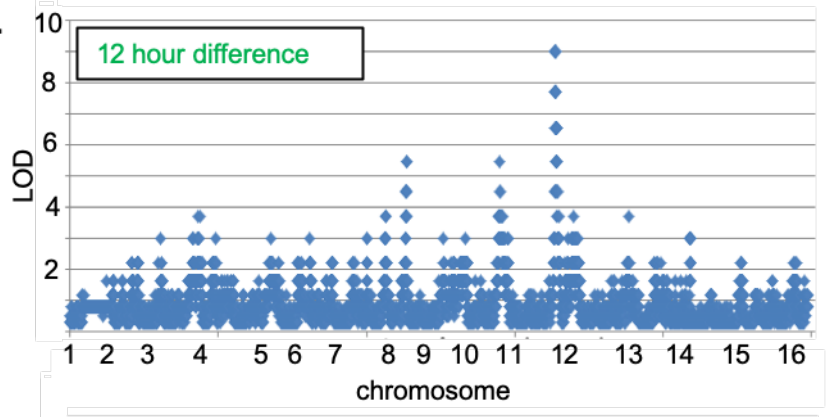

B.

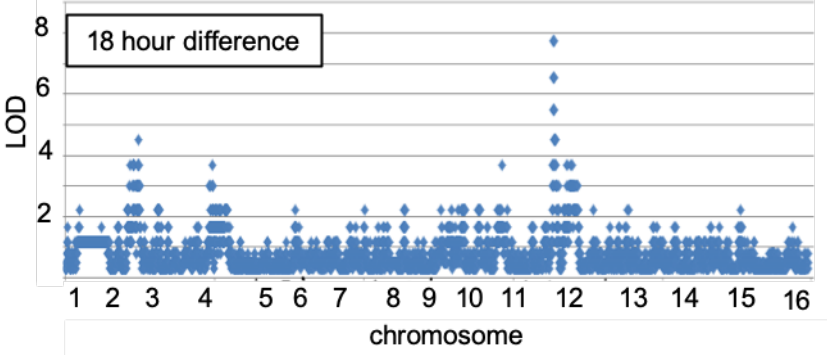

C.

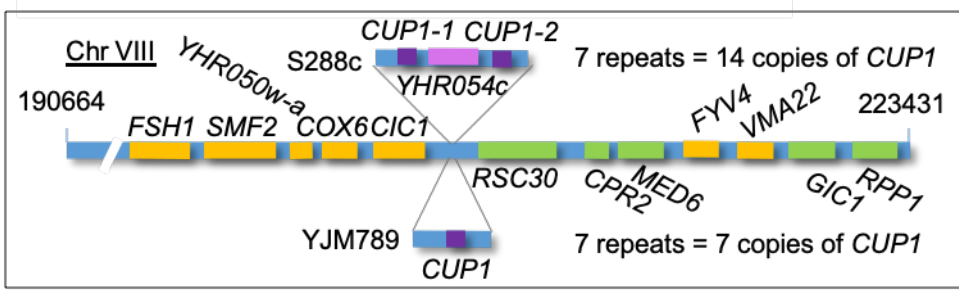

D.

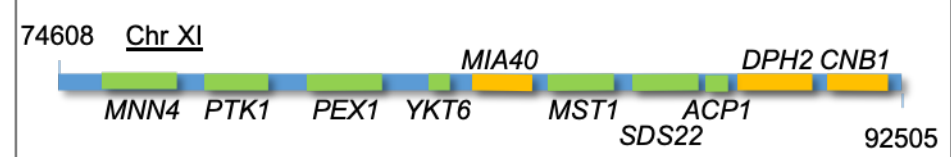

E.

\begin{tabular}{|c|c|c|}
\hline$\frac{\mathrm{Chr} \text { XII }}{\text { YLR125w }}$ & $\begin{array}{l}\text { DCN1 } \\
\quad \text { DIP2/ UTP12 }\end{array}$ & CKI1 PDC5 SLX4 \\
\hline $393415^{Y L R 126 c} A P C 2$ & ZRT2 ACE2 USB1 & 414994 \\
\hline
\end{tabular}

Figure 2- S4. Genomic Loci Linked to Copper Response. A. Genetic linkage with 12-hours of copper treatment. B. Genetic linkage with 18-hours of copper treatment. C. Genes on chromosome XII that are linked to copper response. Chromosomal coordinates are labeled. Green genes are encoded on the top strand and yellow genes are encoded on the bottom strand. C. Genes located on chromosome VIII linked to copper response from 12-hours of growth. Chromosomal coordinates are labeled. Green genes are encoded on the top strand and yellow genes are encoded on the bottom strand. Purple genes are also encoded on the bottom strand within segmentally amplified CUP1 region. D. Genes on chromosome XI that are linked to copper response at 12hours of growth. E. Genes on chromosome XII that were linked to copper response in both 12 and 18-hours of growth. 


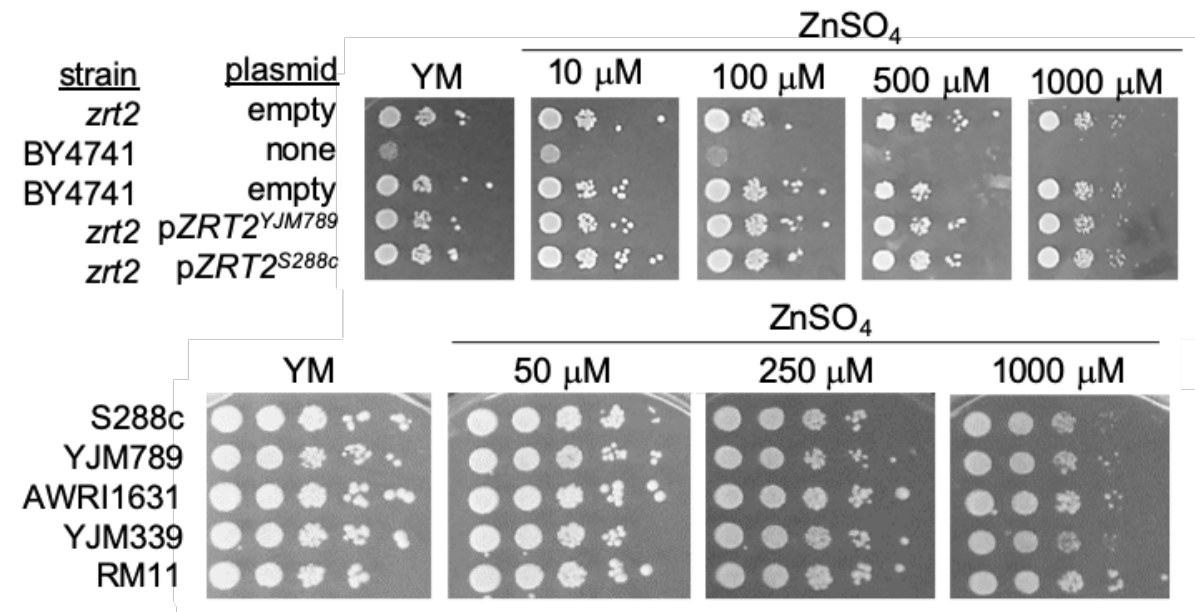

Figure 2- S5. Serial Dilutions of Yeast Growth in the Presence of Elevated Zinc. A. Alleles of Zrt2 were expressed from a plasmid using the endogenous promoter and terminated in BY4741 yeast in which ZRT2 was deleted. Wild-type yeast without a plasmid served as the control for plasmid selection. YM was supplemented with necessary nutrients. B. Genetically diverse yeast S288c (GSY147), YJM789, RM11, AWRI1631 and YJM339 were serially diluted onto varying amounts of zinc sulfate. Plates were incubated for 2-3 days and photographed. 


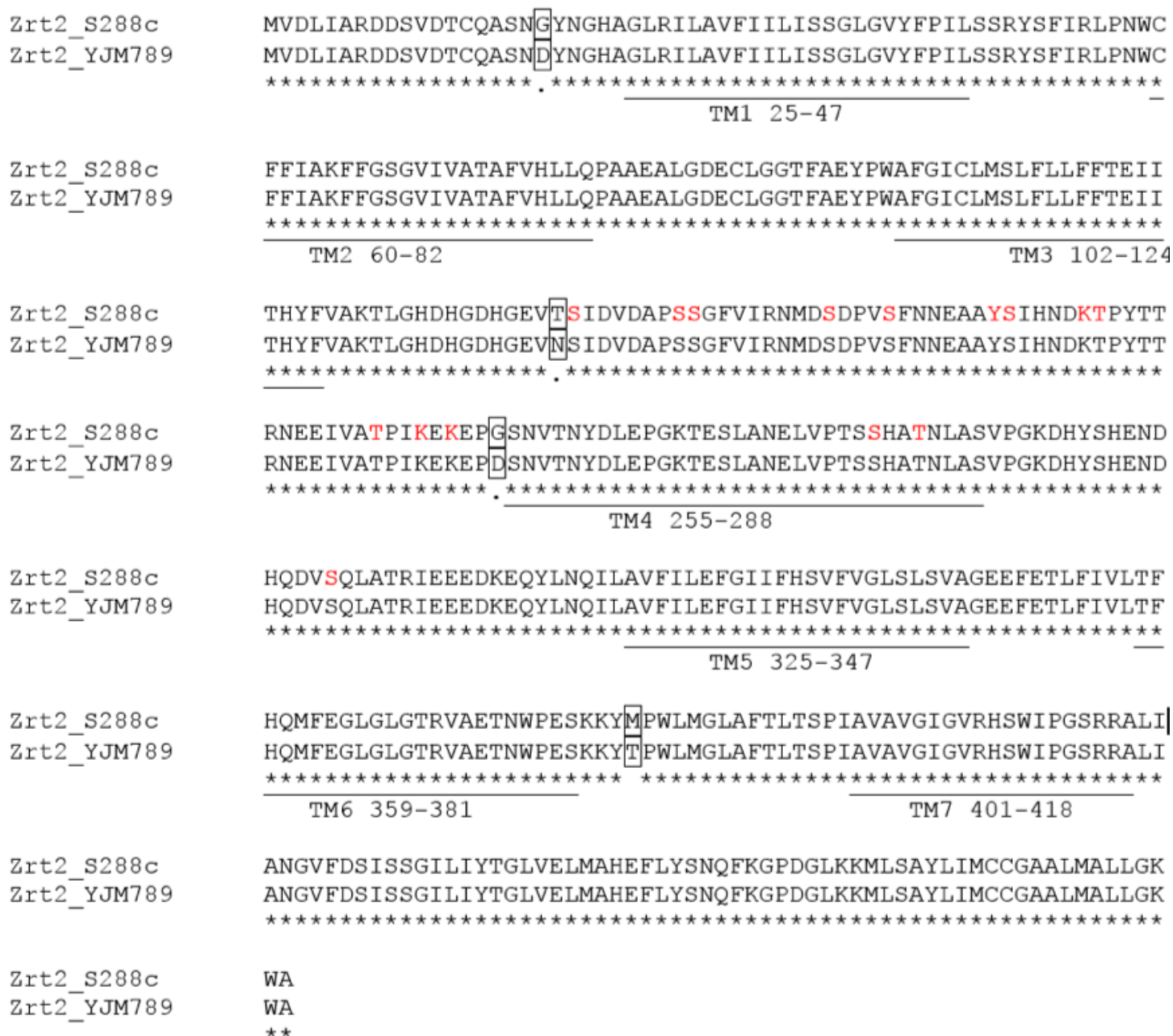

ANGVFDSISSGILIYTGLVELMAHEFLYSNQFKGPDGLKKMLSAYLIMCCGAALMALLGK ANGVFDSISSGILIYTGLVELMAHEFLYSNQFKGPDGLKKMLSAYLIMCCGAALMALLGK

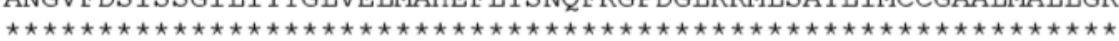

WA

WA

Figure 2- S6. Protein Alignment of Zrt2 from S288c and YJM789. Amino acid that differ are boxed. Below sequences, identical amino acids were signified by $(*)$ and similar amino acids are signified by (.). Transmembrane domain sequences are underlined. Sites of phosphorylation are in red. 


\section{Supplemental Tables}

Table 2-S1. Relative protein levels of S288c (S96) and YJM789 cells grown in YM with lysine or $\mathrm{YM}$ with excess $\mathrm{CuSO}_{4}$, shown as $\log 2$. A total of 112 proteins showed greater than a twofold change between strains and/or copper treatments.

Table 2-S2. Common contaminant list for proteomics that were screened during searches.

Table 2-S3. Growth values of S288c (S96) and YJM789 haploid recombinant segregants at 12 and 18-hours with $50 \mu \mathrm{M} \mathrm{CuSO}_{4}$.

Table 2-S4. SNP LOD mean for 12-hour exposure.

Table 2-S5. SNP LOD mean 18-hour end point exposure.

These supplemental tables can be accessed through this hyperlink. 


\title{
Metallomic and Lipidomic Analysis of Saccharomyces cerevisiae Response to Cellulosic Copper Nanoparticles Uncover Drivers of Toxicity
}

An original research article submitted to Metallomics from the Royal Society of Chemistry

Matthew J. Winans and Jennifer E. G. Gallagher

West Virginia University - Biology Department, 53 Campus Drive LSB 3140, Morgantown, WV, 26506

\begin{abstract}
Nanotechnology is a promising new technology, of which antimicrobial metal nanocomposites are predicted to become valuable in medical and food packaging applications. Copper is a redox-active antimicrobial metal that can become increasingly toxic depending on the target biomolecule's donor atom selectivity and the chemical species of copper present. The carboxymethyl cellulose copper nanoparticles (CMC-Cu) investigated in this study have a unique and undefined toxicity to Saccharomyces cerevisiae that is different from $\mathrm{CuSO}_{4}$. Cellular surface damage was found in scanning electron micrographs upon $\mathrm{CMC}-\mathrm{Cu}$ exposure. Further investigation into the lipids revealed an altered phosphatidylcholine and phosphatidylethanolamine membrane composition, as well as depleted triacylglycerols, suggesting an impact on the Kennedy lipid pathway. High levels of reactive oxygen species were measured which likely played a role in the lipid peroxidation detected with $\mathrm{CMC}-\mathrm{Cu}$ treatment. Metal homeostasis was affected by $\mathrm{CMC}-\mathrm{Cu}$ treatment. The copper sensitive yeast strain, YJM789, significantly decreased cellular zinc concentrations while the copper concentrations increased, suggesting a possible ionic mimicry relationship. Ionic mimicry can occur when a toxic metal replaces or mimics an essential metal such as zinc or essential elements like calcium or magnesium. In contrast to other compounds that generate ROS, we found no evidence of genotoxicity. Mass is the traditional determinant of the intrinsic elemental chemistry, but this practice fails to reflect the morphology and surface area reactivity of nanotechnology. As commonplace objects become more integrated with nanotechnology, humanity must look forward past traditional measurements of toxicity.
\end{abstract}




\section{Introduction}

Copper is known as a broad-spectrum biocidal towards microorganisms ${ }^{1-3}$ and since antiquity, copper vessels were used for water disinfection and food preservation ${ }^{4}$, yet humans have a low contact sensitivity from coinage and personal adornment ${ }^{5}$. In general, antimicrobial metals selectively disrupt cell growth. This growth disruption is influenced by the properties of both the metal and biomolecules. The damage from antimicrobial metal species is spatially localized disrupting membrane function, causing dysfunctional proteins, and DNA damage. Transition metals have the most use as antimicrobials, but toxicity varies between organisms. Important determinants of metal antimicrobial toxicity are donor atom selectivity, speciation, and reduction potential $^{2,6}$. Donor atom selectivity is a factor that influences the compatibility between metal and the ligand that is potentially damaged. Speciation is important because the species of metal has a large influence on its bioavailability and reduction potential, for instance, the difference between $\mathrm{Cu}^{1+}$ and $\mathrm{Cu}^{2+}$. Moreover, potentially toxic materials have several driving factors including their intrinsic chemistry, surface area reactivity, and morphology; which are contributing toxicological factors that influence any material's interaction with organisms.

Not all metals are reduction-oxidation (redox)-active, critically impacting their toxicity. Iron and copper are two transition metals that are well known to be redox active. In biological systems, finely tuned redox activity facilitates the gain or loss of electrons between two chemical species enabling fundamental processes such as respiration, nitrogen fixation, and photosynthesis ${ }^{7}$. During cellular respiration, the mitochondria produce endogenous reactive oxygen species (ROS) as a result of oxygen reduction. This is a consequence of electrons being transferred between complexes to generate an electron potential across the mitochondrial membrane, generating ATP. $\mathrm{H}_{2} \mathrm{O}_{2}$ and $\cdot{ }^{-} \mathrm{O}_{2}$ are products of transferring electrons to molecular oxygen and progenitors of ROS. It is well established that ROS can also act as signaling molecules throughout the cell triggering cell signaling proteins, transporters, ion channels, modifying protein kinase, and ubiquitination systems $^{8}$. Excess copper-generated free radicals damage biomolecules such as cellular membranes, DNA, and functional/structural proteins. Additionally, disruption of the plasma membrane integrity is a major mechanism of copper toxicity in microorganisms and the fatty acid composition of the plasma membrane directly affects $S$. cerevisiae's susceptibility to copper toxicity ${ }^{9}$. Copper ions have been shown to interact with synthetic membrane models of phosphatidylcholine (PC) and phosphatidylethanolamine (PE) in an ordered fashion, first with PC then with PE based on the structure of the membrane prevalent phospholipids ${ }^{10}$.

The metal species determines its reactivity and solubility, key factors in toxicity and bioavailability $^{11,12}$. Inside the cell, essential metals are almost never free because of a plethora of transporters, regulatory sensors, and chaperones that confine the metal species and guide metal atoms to specific sites in proteins ${ }^{7}$. Interactions of metal ions with the atoms of ligand donors, such as functional groups of proteins, are strongly selective ${ }^{13,14}$ and have a bias that facilitates the recognition of correct metal needed for structure and function ${ }^{15}$. Proteins have a somewhat flexible steric selection for their desired metal and their selection is predisposed by an ordered universal preference for essential divalent cations such as copper over zinc ${ }^{16}$. When metal homeostasis is unbalanced some proteins bind incorrect metals, negatively influencing their structure and function. Poorly-discriminating biomolecules contribute to ionic or molecular mimicry by binding with metal ions or metal complexes that resemble their correct cofactors ${ }^{2}$. 
Approximately one-third of all proteins and half of all enzymes are predicted to be dependent on a metal ion for structure or function ${ }^{15,17,18}$. Copper is the third most biologically abundant transition metal after iron and zinc, and unbalanced metal homeostasis results in the mis-metallation of proteins ${ }^{19}$. As an essential micronutrient copper enters $S$. cerevisiae in part via the Ctr family of permeases ${ }^{20}$. Under low copper conditions, Ctr1 and Ctr3 form a high-affinity copper importation system on the plasma membrane. Environmental copper, $\mathrm{Cu}^{2+}$, is reduced by metalloreductases to $\mathrm{Cu}^{1+}$. Ctr1 harbors $\mathrm{Cu}^{1+}$ in a trimeric channel complex facilitated by the ectodomain containing multiple MxxM sequence motifs to scavenge the copper ions for transport across the plasma membrane $^{21}$. The low-affinity transport of copper is facilitated by permeases Fet 4 and Smf1 ${ }^{20,22,23}$. The metallochaperones Atx1 and Ccsl transport $\mathrm{Cu}^{1+}$ to sites of utilization, such as target cuproenzymes $^{24,25}$. Ccs1 transfers $\mathrm{Cu}^{1+}$ to the Apo-protein Sod1 forming an essential disulfide bond ${ }^{26,27}$. Atx 1 delivers $\mathrm{Cu}^{1+}$ to the trans-Golgi network where proteins are metallated prior to dissemination ${ }^{28,29}$. While there are many investigations into the mitochondrial transport components, how the mitochondrial copper pool is assembled is not completely understood. In copper-deficient environments, Mac1 activates Fre1 and Ctr1 forming a high-affinity copper import system ${ }^{30,31}$. In high concentrations of copper, Ace1, also known as the transcription factor Cup2, activates transcription of genes encoding metallothioneins such as Cup1 and Sod1 ${ }^{32-34}$. Cup1 is the major copper activated metallothionein that mediates copper-induced oxygen radical detoxification $^{35,36}$. Between strains of $S$. cerevisiae, variation in the number of genomic amplifications is common and generally, an increased copy number increases copper resistance ${ }^{37}$. Mac1 and Ace1, transcription factors, work together by targeting opposing functions in maintaining a proper copper balance. Moreover, the Zrt- and -Irt like protein (ZIP) family of transporters have been found to facilitate direct metal importation by these mechanisms ${ }^{38,39}$ while co-transportation of metals with ligands has also been shown to be a source of metal accumulation $^{40}$. Yeast cells accumulate surplus metals in vacuoles ${ }^{41,42}$ and intracellular accumulation of metal is routinely the first step in metal poisoning ${ }^{6}$.

Cellulose is a renewable polymer resource of unmatched abundance and its fibers or derivatives have been used in daily life for many generations. It is a fibrous, hardy, water-insoluble substance composed of a high molecular weight homopolymer of $\beta$-1,4-linked anhydro-D-glucose units that maintain the structure of cell walls in plants ${ }^{43}$. Historically, cellulose has been regarded as benign ${ }^{44}$ to biological systems; however, it is toxicologically understudied compared to asbestos and manmade mineral fibers ${ }^{45}$. The high number of Na-carboxyl groups on carboxymethyl (CMC) make it an attractive organic support structure on which to construct copper nanoparticles because it can facilitate copper's reduction and therefore further copper's availability on the cellulose structure $^{46,47}$. The CMC-Cu nanomaterials are considered a hybrid material, composed of copper and cellulose, distinct from metallic nanoparticles. They are synthesized in situ and reduced onto available carboxylic groups of carboxymethyl cellulose (CMC) as a template and stabilizer in order to manufacture nanocomposite materials ${ }^{47}$. These $\mathrm{CMC}$ microfibril strands contain the reduced copper nanoparticles (CuNP) that exhibit mostly $\mathrm{Cu}^{1+}$ and $\mathrm{Cu}^{0}$ forms averaging $15 \mathrm{~nm}$ in diameter ${ }^{47}$. CuNPs are built on $\mathrm{CMC}$, forming $\mathrm{CMC}-\mathrm{Cu}$. Variations in the copper composition and cellulose biopolymer have been successfully synthesized resulting in changes to their interaction with microorganisms ${ }^{48-51}$. Saccharomyces cerevisiae and Escherichia coli are susceptible to cell death upon $\mathrm{CMC}-\mathrm{Cu}$ exposure ${ }^{36,47}$. $\mathrm{CMC}-\mathrm{Cu}$ sensitivity mirrors the sensitivity of genetically diverse yeast exposed to soluble copper in $\mathrm{CuSO}_{4}$ form ${ }^{36}$. Unlike zinc or titanium oxides, when 
copper oxide is presented to yeast on the nanoscale, it is approximately 60 -fold more toxic than its soluble counterpart ${ }^{52}$. Previous studies in higher eukaryotes found that CuO NPs have a "Trojan horse mechanism" that induces endocytosis and causes DNA damage by the intracellular ionic release of copper ${ }^{53}$. CuNPs seem to target the cell membrane to cause cellular death by loss of membrane integrity ${ }^{54}$.

Aqueous copper exists in the particulate, colloidal, nanoparticle, and soluble states, predominately as metal and $\mathrm{Cu}^{2+}$ ions ${ }^{6,55}$. S. cerevisiae was chosen as a model system to investigate the nanotoxicity of the CMC-Cu exposures because of its genetic tractability, conserved genome, and exceptional wealth of experimental resources ${ }^{56}$. This budding yeast has a small ellipsoid, 60 - 30 $\mathrm{nm}$ in diameter, endocytic vesicle ${ }^{57}$. Endocytosis internalizes cargo from the plasma membrane and has a critical role in nutrient uptake, damaged protein turnover, membrane composition, and the response to extracellular signals. Yeast contains a family of arrestins that have been suggested as a general model for transporter regulation ${ }^{58,59}$. Yeast $\alpha$-arrestins are intracellular proteins that target specific plasma membrane proteins for the endocytic system ${ }^{60}$ including metal transporter $\mathrm{Smfl}^{59}$. Once arrestins associate with plasma membrane proteins, ubiquitin-conjugating proteins are recruited. Ubiquitinated cargo is claimed for endocytosis and transfers into the vacuole for degradation via both clathrin-independent and clathrin-mediated endocytosis ${ }^{61}$. Plasma membrane surface remodeling can be triggered by cellular metabolism and extracellular cues, perhaps to include copper availability. The organization and proper composition of the plasma membrane are maintained by endocytic downregulation of plasma membrane proteins during surface remodeling.

This study investigated the influence of CMC-Cu exposures to $S$. cerevisiae on metal homeostasis, ROS production, lipid interaction, cellular morphology, and genotoxicity. Taken together with an initial study characterizing $\mathrm{CMC}-\mathrm{Cu}$ sensitivity owing from genetic diversity, location, and genetic contributors we present an intricate nanotoxicological profile of $S$. cerevisiae post $\mathrm{CMC}-\mathrm{Cu}$ exposure. Copper nanoparticles derived from $\mathrm{CMC}-\mathrm{Cu}$ treatments caused toxicity and cellular death that was different from soluble copper sulfate ${ }^{36}$. This study found an increase in intracellular ROS that lacked evidence of genotoxicity, but supported lipid peroxidation from $\mathrm{CMC}-\mathrm{Cu}$ exposure in yeast. Further examination found the lipid profiles not only changed in response to $\mathrm{CMC}-\mathrm{Cu}$, but also showed $\mathrm{CMC}$ alone affected the lipid composition. Lipid damage has been associated with copper's antimicrobial mode of toxicity and copper has been found to interact with the major membrane phospholipid bilayer components, $\mathrm{PE}$ and $\mathrm{PC}$, which supports our findings ${ }^{10,62,63}$. Inspection of the cellular morphology unveiled a disfigurement after $\mathrm{CMC}-\mathrm{Cu}$ exposure. At the current juncture, there are insufficient findings to definitively assign this disfigurement as causative or consequential, but taken together this evidence suggests that cellular membrane damage is a primary target of $\mathrm{CMC}-\mathrm{Cu}$ damage. Additionally, metal homeostasis is also affected, resulting in the displacement of $\mathrm{Zn}$ possibly via mis-metallation or ionic mimicry.

\section{Methods and Materials}

\section{Cellular Metals}

Yeast strains BY4741 and YJM789 were grown in triplicate until log-phase in YM+HULM (histidine, uracil, leucine, and methionine) or $\mathrm{YM}$ at $30^{\circ} \mathrm{C}$, respectively. Yeast cells were treated with $400 \mu \mathrm{M} \mathrm{CuSO} 4$ or $157 \mu \mathrm{M} \mathrm{CMC}-\mathrm{Cu}$ for 90 minutes. An equal number of cells were determined by optical density at $600 \mathrm{~nm}$ to collect equivalent to 5 optical density units (ODu) of 
cells. These samples were centrifuged and washed twice with distilled water. One ODu was separated for protein quantification. Samples were split, frozen in liquid nitrogen, and stored at $80^{\circ} \mathrm{C}$. Soluble protein concentration was determined via Bradford assay as previously published 36. For inductively coupled plasma - optical emission spectroscopy (ICP-OES), the cell pellets were treated with $600 \mu \mathrm{L}$ of concentrated $\mathrm{HNO}_{3}$ and $200 \mu \mathrm{L}$ of $30 \% \mathrm{H}_{2} \mathrm{O}_{2}$ for digestion ${ }^{64}$. These solutions were transferred to glass tubes and placed in a boiling water bath for 2 hours until clear. The liquids were filtered via syringe and readjusted to their original volumes with distilled water. Samples were analyzed at $327.395 \mathrm{~nm}$ for $\mathrm{Cu}$ and at $213.857 \mathrm{~nm}$ for $\mathrm{Zn}$. Metal concentrations were given in $\mathrm{mg} \mathrm{ml}^{-1}$ and normalized to soluble protein concentration for each sample. Three biological replicates were used in this protocol. ANOVA statistical analysis was utilized in determining significance with a Tukey-HSD post-hoc analysis. An alpha of 0.05 was used as the cutoff for significance. SAS JMP (SAS JMP Pro Version 13, SAS Institute Inc., Cary, NC, USA) was used for statistical software. The standard error of the mean was used in graphing error bars.

\section{Cellular ROS}

Cellular ROS detection assay was carried out with modification to an existing protocol ${ }^{65}$. The 2,7dichlorofluorescin diacetate (DCFDA) cellular ROS detection assay kit was ordered from Abcam (ab113851). Briefly, yeast strain BY4741 was grown to stationary-phase in YPD supplemented with DCFDA without light at $30^{\circ} \mathrm{C}$. Cultures were centrifuged and washed in phosphate-buffered solution before being resuspended YM+HULM to a final $\mathrm{OD}_{600}$ of 0.7 . Yeast were treated for 90 minutes, $\mathrm{CMC}-\mathrm{Cu}$ at $157 \mu \mathrm{M}$ and $\mathrm{CuSO}_{4}$ at $400 \mu \mathrm{M}$, and controls, $\mathrm{H}_{2} \mathrm{O}_{2}$ at $489 \mu \mathrm{M}$, in a 96 well black bottom microplate from Greiner Bio-One for end-point fluorescence measurement at Ex/Em $=485 / 535 \mathrm{~nm}$ on a SpectraMax Gemini XPS Fluorescence Microplate Reader from Molecular Devices. Five biological replicates were used in this protocol. ANOVA statistical analysis was utilized in determining significance with a Tukey-HSD post-hoc analysis. An alpha of 0.05 was used as the cutoff for significance. SAS JMP (SAS JMP Pro Version 13, SAS Institute Inc., Cary, $\mathrm{NC}$, USA) was used for statistical software. The standard error of the mean was used in graphing error bars.

\section{Lipid Peroxidation}

Lipid peroxidation was colorimetrically measured by assessing the major byproduct of lipid peroxidation, malondialdehyde, in a Thiobartic Acid Reactive Substances assay (TBARS). The OxiSelect ${ }^{\mathrm{TM}}$ TBARS assay kit (STA-330) was obtained from CellBioLabs, Inc, San Diego, CA, USA. Yeast strain YJM339 was grown in triplicate until $\log$ phase in $\mathrm{YM}$ at $30^{\circ} \mathrm{C}$. Yeast cells were harvested at a final $\mathrm{OD}_{600}$ of 0.7 , treated with $400 \mu \mathrm{M} \mathrm{CuSO} 4,157 \mu \mathrm{M} \mathrm{CMC}-\mathrm{Cu}$, or $498 \mu \mathrm{M}$ $\mathrm{H}_{2} \mathrm{O}_{2}$ for 90 minutes. A kit-supplied SDS lysis solution (Part No. 233003) was incubated within both the samples and the malondialdehyde (MDA) standards (Part No. 233001) at 1:1 ratio totaling $200 \mu \mathrm{l}$ for five minutes at $23^{\circ} \mathrm{C}$. $250 \mu \mathrm{l}$ of thiobartic acid reagent $\left(5.2 \mathrm{mg} \mathrm{mL}^{-1}\right.$ at $\left.\mathrm{pH} 3.5\right)$ (Part Nos. $233002 \& 233004$ ) was added to each sample and incubated at $95^{\circ} \mathrm{C}$ for 50 minutes. Samples were cooled in an ice bath for five minutes before 15 minutes of centrifugation at 3,000 g. The supernatant was harvested and aliquoted for duplicate technical spectrophotometric measurement in a TECAN Infinite 200 pro microplate reader at $532 \mathrm{~nm}$ absorbance. TBARS levels were determined from an MDA equivalence standard curve ranging from 0 to $125 \mu \mathrm{M}$. ANOVA statistical analysis was performed with Tukey-HSD post-hoc analysis. An alpha of 0.05 was used as the cutoff for significance. SAS JMP (SAS JMP Pro, Version 13, SAS Institute Inc., Cary, NC, USA) was used for the construction of the whisker-box plots. 


\section{Electron Microscopy}

Yeast strain BY4741 was grown in YM+HULM until log-phase at $30^{\circ} \mathrm{C}$. Cells were treated with $\mathrm{CMC}-\mathrm{Cu}$ at $400 \mu \mathrm{M}$ for 90 minutes before $6 \mathrm{ml}$ were harvested by centrifugation for 4 minutes at $3,000 \mathrm{~g}$. The supernatant was pipetted off and the cells were resuspended in $500 \mu \mathrm{l}$ of paraformaldehyde (14\%) for one hour at room temperature. Fixed cells were washed once and resuspended in $0.1 \mathrm{M} \mathrm{KPO} 4 / 1.2 \mathrm{M}$ sorbitol buffer for storage at $4^{\circ} \mathrm{C}$. Within 72 hours in the WVU Electron Microscopy Facility, the cells were fixed with 2.5\% glutaraldehyde for one hour before washing thrice with PBS for 15 min each. Samples were then aliquoted into Eppendorf tubes for either SEM or TEM processing and imaging.

The cells were dehydrated stepwise by 15 min ethanol baths starting at $30 \%$ and increasing to $50 \%$, $70 \%, 90 \%$, and finally, $100 \%$. The $100 \%$ ethanol bath was repeated three times. Yeast cells were pelleted and the supernatant was discarded after each ethanol addition. Samples were submerged in hexamethyldisilazane to dry the cells for 15 minutes before allowing the cells to air-dry overnight in a fume hood with the caps of the Eppendorf tubes slightly open. Samples were adhered to pins by carbon tape and sputtered with gold and pallidum (60:40) for 150 seconds at $18 \mathrm{kV}$ with rotation. A Denton Desk V Sputter and Carbon Coater were used in sputtering samples. Images were captured on a Hitachi S-4700 Scanning Electron Microscope.

\section{DNA Damage}

Damage to DNA and chromosomes was assessed by pulse-field gel electrophoresis (PFGE). Yeast strain BY4741 was grown to $\log$ phase in YM+HULM media at $30^{\circ} \mathrm{C}$. Cells were treated with $\mathrm{H}_{2} \mathrm{O}_{2}$ at $489 \mu \mathrm{M}$ or CMC-Cu $400 \mu \mathrm{M}$ up to 90 minutes. One $\mathrm{OD}_{600 \mathrm{~nm}}$ unit of yeast cells was harvested by centrifugation in conical tubes at time points of $5 \mathrm{~min}, 15 \mathrm{~min}, 30 \mathrm{~min}, 60 \mathrm{~min}$, and $90 \mathrm{~min}$. The sample supernatant was removed and frozen in liquid nitrogen for storage at $-80^{\circ} \mathrm{C}$, sample processing occurred within 24 hours. Cells and zymolase enzymes at a final concentration of $0.05 \mu \mathrm{g} / \mathrm{ml}$ were mixed $1: 1$ with $2 \%$ low melting agarose gel diluted in $0.5 \mathrm{x}$ TBE to obtain 1 OD unit per plug. Plugs were made in a Bio-Rad disposable plug mold via heat block set to $50^{\circ} \mathrm{C}$ with a cut off pipette tip to facilitate the agarose mixing. The solidification of agarose plugs occurred for $15 \mathrm{~min}$ at $4^{\circ} \mathrm{C}$. To digest the cell wall, $1 \mathrm{ml}$ of plugs was placed in $5 \mathrm{ml}$ of M/15 PBS supplemented with zymolase and incubated for 1 hour at $37^{\circ} \mathrm{C}$ without agitation. Plugs were washed with a wash buffer (20mM Tris, $50 \mathrm{mM}$ EDTA, $\mathrm{pH} 8)$. The protein was digested by adding a proteinase $\mathrm{K}$ buffer $(30 \mathrm{mM}$ Tris, $50 \mathrm{mM}$ EDTA, $1 \% \mathrm{SDS}, 0.125 \mu \mathrm{g} / \mathrm{ml}$ proteinase $\mathrm{K}, \mathrm{pH} 8)$ and incubating at $50^{\circ} \mathrm{C}$ overnight. Plugs were washed four times for 1 hour each at $23^{\circ} \mathrm{C}$. Plugs were trimmed to $3 / 4$ original size, positioned onto the comb ends and adhered with one droplet of low melting agarose. A 1.5\% PFGE gel was made using 0.5x TBE and Bio-Rad PFGE agarose by casting into a mold with the comb in position. After the gel solidified, the comb was removed and the gel was placed into a Bio-Rad CHEF DR II system filled with $0.5 \mathrm{x}$ TBE equipped with a water cooler. Electrophoresis of the experimental gel occurred for 24 hours at $14^{\circ} \mathrm{C}$ with a switch time of $60-120$ seconds and set to $6 \mathrm{v} / \mathrm{cm}$. The gel was removed, stained in a $0.125 \mu \mathrm{g} / \mathrm{ml}$ ethidium bromide bath for 10 minutes, and destained with distilled water for one hour before imaging at $254 \mathrm{~nm}$. Pictures were manipulated via cropping in Microsoft PowerPoint solely to straighten running lanes for added clarity. 


\section{Exploration of Lipidome}

Lipid metabolites were assessed in $S$. cerevisiae YJM789, grown in YM to exponential phase (OD $0.4-0.6$ ), and then treated or not for 90 minutes. Six independent biological replicates were performed. Five ODu of cells were harvested, washed with MQ (milliQ) water, and flash-frozen in liquid nitrogen for storage at $-80^{\circ} \mathrm{C}$ until extraction within 24 hours. Lipid and polar metabolites were extracted with $6 \mathrm{ml}$ of a 1:2:0.8 mixture of chloroform: $\mathrm{MeOH}: \mathrm{H}_{2} \mathrm{O}$, following a modified version of a published protocol ${ }^{66,67}$. Glassware was used to avoid polymer contamination. Extraction occurred in 15 mL Kimble ${ }^{\mathrm{TM}}$ Kontes $^{\mathrm{TM}} \mathrm{KIMAX}^{\mathrm{TM}}$ reusable High Strength Centrifuge Tubes from Fisher Scientific. HPLC-grade chloroform and methanol were from Sigma-Aldrich. Six replicates of $3 \mathrm{ml}$ each were harvested for their lipid phase and dried under nitrogen gas. The lipid phases were re-suspended in $500 \mu \mathrm{L}$ 1:1 chloroform: $\mathrm{MeOH}$ and these extracts were stored at $-20^{\circ} \mathrm{C}$ until analysis within 48 hours.

Lipid extracts were analyzed by direct injection using a Thermo Fisher Scientific QExactive, with an ESI (electrospray ionization), using positive and negative modes. For lipid compounds in positive mode, the injection speed was $10 \mu \mathrm{L} / \mathrm{min}$, the scan range: $150.0-2,000.0 \mathrm{~m} / \mathrm{z}$, no fragmentation, 140,000 resolution, 1 microscan, AGC target $5 \times 10^{5}$, maximum injection time of 100 , sheath gas flow rate of 15 , aux gas flow rate of 11 , no sweeping gas flow, spray voltage of $3.50 \mathrm{kV}$, capillary temperature of $300^{\circ} \mathrm{C}$, S-lens RF level: 25.0. These same parameters were kept for lipid compounds in negative mode except for a spray voltage of $3.20 \mathrm{kV}$. For each sample, a total of 50 scans were obtained and averaged with Thermo Scientific Xcalibur 2.1 SP1. Averaged spectra in the positive and negative mode were processed with $\mathrm{xcms} 3.2 .0^{68}$. Peaks were identified within each spectrum using the mass spec wavelet method from the MassSpecWavelet 1.46.0 R package ${ }^{69}$. Peaks were grouped via the Mzclust method, followed by group ChromPeaks. All features were plotted and visually inspected. Intensity values of each sample feature were obtained via the featureValue method using the integrated signal area for each representative peak per sample. Feature intensity and feature definition tables were saved as CSV files. Features were identified via MetaboSearch $1.2^{70}$. The query ID list was comprised of the average mz values for each feature in positive or negative mode with $5 \mathrm{ppm}$ of error and was searched against the four databases available online in the program: Human Metabolome Database (HMDB) ${ }^{71}$, Metlin $^{72}$, Madison Metabolomics Consortium Database (MMCD) ${ }^{73}$ and LipidMaps ${ }^{74}$. Identifications were manually cross-referenced with Yeast Metabolome Database (YMDB) ${ }^{75}$ and PubChem ${ }^{76}$. Feature intensity tables were composed of only identified features from positive and negative modes with at least five biological replicates. MetaboAnalyst ${ }^{77}$ was used for statistical analysis and generation of the graphical figures. The experiment was repeated twice with consistent results.

\section{Results and Discussion}

\section{Metal Homeostasis}

To explore compromised metal homeostasis induced by $\mathrm{CMC}-\mathrm{Cu}$ exposure, the intracellular metal concentrations were quantified. Experimentally, the Zrt1 protein levels in copper resistant yeast S288c increased 2-fold in response to copper perturbation, suggesting some responsive role for the Zrt transporters ${ }^{36}$. Cellular copper and zinc levels were analyzed via inductively coupled plasma- 
optical emission spectroscopy (ICP-OES) after exposure to $400 \mu \mathrm{M} \mathrm{CuSO}_{4}$ or $157 \mu \mathrm{M}$ $\mathrm{CMC}-\mathrm{Cu}$ which results in nearly equal growth inhibition of the copper sensitive $\operatorname{strain}^{36}$. During normal growth of the sensitive clinical isolate YJM789 in YM, ICP-OES analysis measured $0.043 \mathrm{mg} \mathrm{mL}^{-1}$ $\mathrm{Cu}$, standard error (SE) 0.011 (Figure 3-1A and Figure 3-S1).The resistant agricultural/lab isolate BY4741's copper content was undetectable (Figure 3-1B). Untreated cells of both resistant and sensitive strains were statistically the same. Unsupplemented YM medium acts as a sufficient copper source at $0.16 \mathrm{nM} \mathrm{Cu}$. After $\mathrm{CuSO}_{4}$ addition, copper levels increased for each strain. The level of copper in YM is not optimal for BY4741 because the addition of $50 \mu \mathrm{M} \mathrm{CuSO} 4$ increases growth ${ }^{36}$. With $\mathrm{CuSO}_{4}$, copper levels rose but to differing degrees. YJM789 had $1.223 \mathrm{mg} \mathrm{mL}^{-1} \mathrm{Cu}, \mathrm{SE}$ 0.011 and BY4741 had $0.837 \mathrm{mg} \mathrm{mL}^{-1} \mathrm{Cu}$, SE 0.029. Copper levels increased in the resistant strain, BY4741, to $2.541 \mathrm{mg} \mathrm{mL}^{-1}$ $\mathrm{Cu}$ after $\mathrm{CMC}-\mathrm{Cu}$ treatment. Tukey-Kramer honestly significant difference (Tukey-HSD) post-hoc statistical analysis showed no statistical difference between copper levels of either $\mathrm{CMC}-\mathrm{Cu}$ or $\mathrm{CuSO}_{4}$ treated cells of either strain with one exception (Table 1). The sensitive strain, YJM789, increased its copper concentration to $7.230 \mathrm{mg} \mathrm{mL}^{-1} \mathrm{Cu}$, SE 1.086 after treatment with $\mathrm{CMC}-\mathrm{Cu}$. to $\mathrm{CMC}-\mathrm{Cu}(\mathrm{p}<0.001)$.
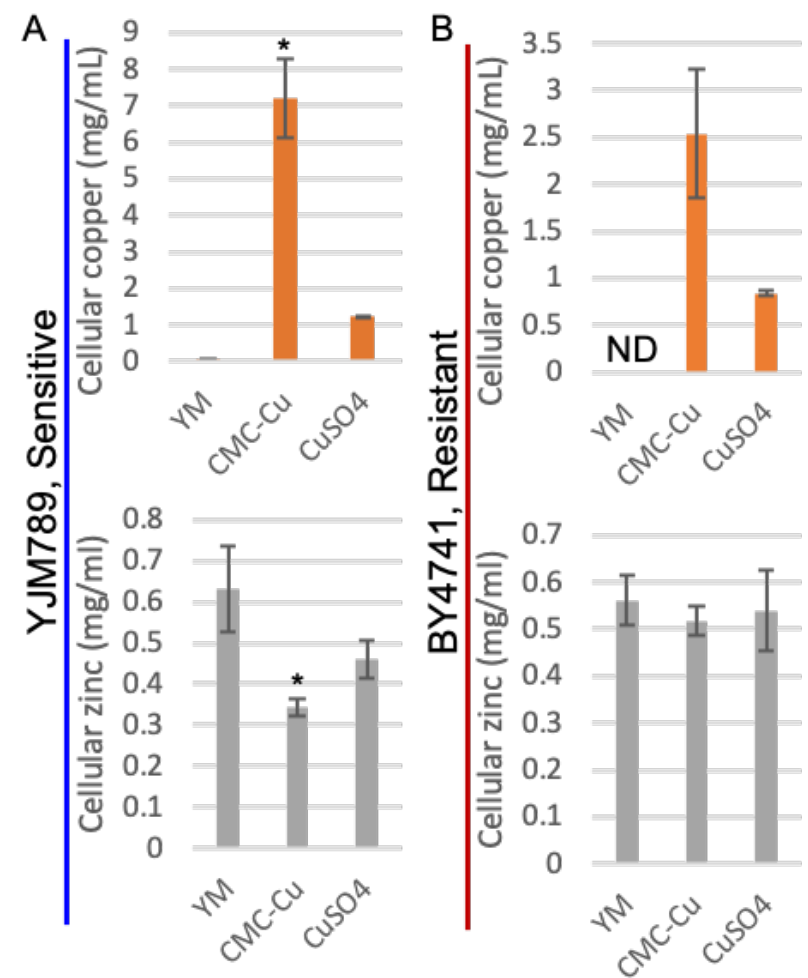

Figure 3-1. Copper and Zinc Metal Homeostasis Measured by Inductively Coupled Plasma - Optical Emissions Spectroscopy During CMC-Cu Toxicity. Sensitive strain YJM789 (A) and resistant strain BY4741 (B) were harvested after 90-minute treatments with CMC$\mathrm{Cu}, \mathrm{CuSO} 4$, or in YM media. (A) YJM789 has a significant increase in cell-associated copper with $\mathrm{CMC}-\mathrm{Cu}$ treatment and a decrease in cellular zinc. (B) BY4741 showed an increase of cellular copper with $\mathrm{CMC}-\mathrm{Cu}$ treatment, no change to zinc concentrations. Statistical analysis performed via one-way ANOVA with a post hoc Tukey HSD analysis $(\mathrm{p}=0.05) . \mathrm{ND}=$ Not detected.

Zinc is a divalent transition metal that binds to ligands less stably than copper according to the Irving-Williams series ${ }^{16}$. The concentration of cellular zinc significantly decreased from the YM baseline of $0.632 \mathrm{mg} \mathrm{mL}^{-1}$ zinc for yeast strain YJM789 in response to CMC-Cu treatment, resulting in a cellular concentration of $0.344 \mathrm{mg} \mathrm{mL}^{-1} \mathrm{Zn}$, SE $0.0449(\mathrm{p}=0.028)$. In response to CMC-Cu treatment, the sensitive strain, YJM789, increased levels of copper while it decreased the levels of zinc. This evidence suggests that metal homeostasis of zinc and copper influences the toxicity of $\mathrm{CMC}-\mathrm{Cu}$. Zinc metallates 582 potential zinc-binding proteins contributing to the 
structure and function of approximately $\sim 10 \%$ of the total yeast proteome ${ }^{19}$. S. cerevisiae yeast have a minimum zinc quota of $\sim 10^{7}$ atoms $/$ cell ${ }^{78}$, most of which is tightly controlled allowing a low level of labile zinc ${ }^{79-81}$. This labile zinc is maintained via intercellular storage in organelles, in the cytosol by dynamic metallothioneins that buffer redox activity, and in intracellular yeast vesicle compartments that resemble mammalian zincosomes ${ }^{82-84}$. The cellular zinc levels of the other conditions and strains were all statistically similar. After $\mathrm{CuSO}_{4}$ treatment, YJM789 zinc levels decreased from $0.632 \mathrm{mg} \mathrm{mL}^{-1}$ to $0.46 \mathrm{mg}$ $\mathrm{mL}^{-1}$. BY4741, the copper resistant strain, in YM had $0.562 \mathrm{mg} \mathrm{mL}^{-1}$ of zinc. CMC-Cu treatment did not significantly change the levels of zinc nor did $\mathrm{CuSO}_{4}$ cause changes in $\mathrm{Zn}$ concentrations in the copper resistant strain. The levels of zinc in YJM789 treated with $\mathrm{CMC}-\mathrm{Cu}$ decreased to nearly half compared to the yeast grown in YM. The findings presented here align with literature reports implicating a role for zinc in copper nanoparticle toxicity ${ }^{36}$. The compromised metal homeostasis observed in YJM789 is likely involved in this strain's sensitivity towards copper. One possible explanation of the relationship seen between falling $\mathrm{Zn}$ and increasing $\mathrm{Cu}$ levels involves the stability of transition-metal complexes as described by the Irving-Williams series. In this trend, divalent transition metals create an increasingly stable complex resulting with copper as the most favored. The ligands selection bias is as follows: $\mathrm{Mn}^{2+}<\mathrm{Fe}^{2+}<\mathrm{Co}^{2+}<\mathrm{Ni}^{2+}<\mathrm{Cu}^{2+}>\mathrm{Zn}^{2+16}$ and this is related to size and charge of the metals. As seen in other reports, one way this unbalance may propagate is through ionic mimicry, where zinc or other transporters select copper instead of zinc ${ }^{85}$. This chemical tendency between metal atoms not only is a factor of CMC-Cu toxicity, but also is a factor in antimicrobial metal toxicity.

\section{Intracellular Reactive Oxygen Species}

As a redox-active metal, copper is well-known to proliferate ROS, and this study sought to investigate ROS as a toxicological mechanism of $\mathrm{CMC}-\mathrm{Cu}$ exposure. Endogenous ROS is produced through aerobic respiration giving rise to incompletely reduced forms of molecular oxygen $\left(\mathrm{O}_{2}\right)$ which yield hydrogen peroxide $\left(\mathrm{H}_{2} \mathrm{O}_{2}\right)$ and hydroxyl radical $\left(\cdot{ }^{-} \mathrm{O}_{2}\right)$. In the presence of a redox-active metal, Fenton reaction driven autooxidation occurs. To measure intracellular ROS, a fluorometric assay utilizing 2,7-dichlorofluorescin diacetate (DCFDA) was employed. It was found that $\mathrm{CMC}-\mathrm{Cu}$ treatments of $157 \mu \mathrm{M}$ produced high amounts of ROS measured at 11.35 fluorescence (FC), SE 0.26 (Figure 3-2A); nearly double the endogenous ROS levels in YM grown yeast. Hydrogen peroxide $\left(\mathrm{H}_{2} \mathrm{O}_{2}\right)$ treatments of $489 \mu \mathrm{M}$ produced similar levels of intracellular ROS of 10.38 FC, SE 0.26. Although soluble and nano-copper treatments were determined to inhibit growth at similar levels ${ }^{36}$, the $400 \mu \mathrm{M}$ treatment of $\mathrm{CuSO}_{4}$ did not produce significant enough levels of ROS via this DCFDA assay. The yeast used here, BY4741, tolerated $400 \mu \mathrm{M}$ $\mathrm{CuSO}_{4}$ well. It is likely that increased ROS detection would occur by exposing this yeast to increased levels of $\mathrm{CuSO}_{4}$, which would increase the growth inhibition of this resistant strain. $\mathrm{CuSO}_{4}$ treatments produced intracellular ROS of 5.62 FC, SE 0.26 which were similar to the level 
detected in untreated control cells. ROS was detected at 5.5 FC, SE 0.26 in untreated cells (YM). Tukey-HSD post-hoc statistical analysis defined three detection levels between our samples, a low-level ROS group of $\mathrm{CuSO}_{4} / \mathrm{YM}$ and two different high-level ROS groups composed of either $\mathrm{H}_{2} \mathrm{O}_{2}$ or CMC-Cu. Reduction potential is a key factor when determining the toxicity of antimicrobial metals. In bacteria, there have been at least three mechanisms suggested for the increased ROS produced via metal poisoning. They include catalyzing Fenton chemistry ${ }^{86,87}$, disruption of iron ligands such as Fe-S clusters that release redox-active $\mathrm{Fe}^{88,89}$, and thiol mediated reduction of metal species ${ }^{87,90}$. Importantly, the oxidation of cellular thiols occurs via $\mathrm{Cu}-\mathrm{S}$ covalent bonding which leads to disulfide bonds in proteins, thus depleting critical antioxidants including glutathione, ultimately preventing cellular repair $^{2,91-93}$. The glutathione tripeptide molecule contains thiol ligand groups in their cysteine amino acid. This sulfur-containing molecule reduces copper by donating an electron to copper, which results in oxidation of the thiol group
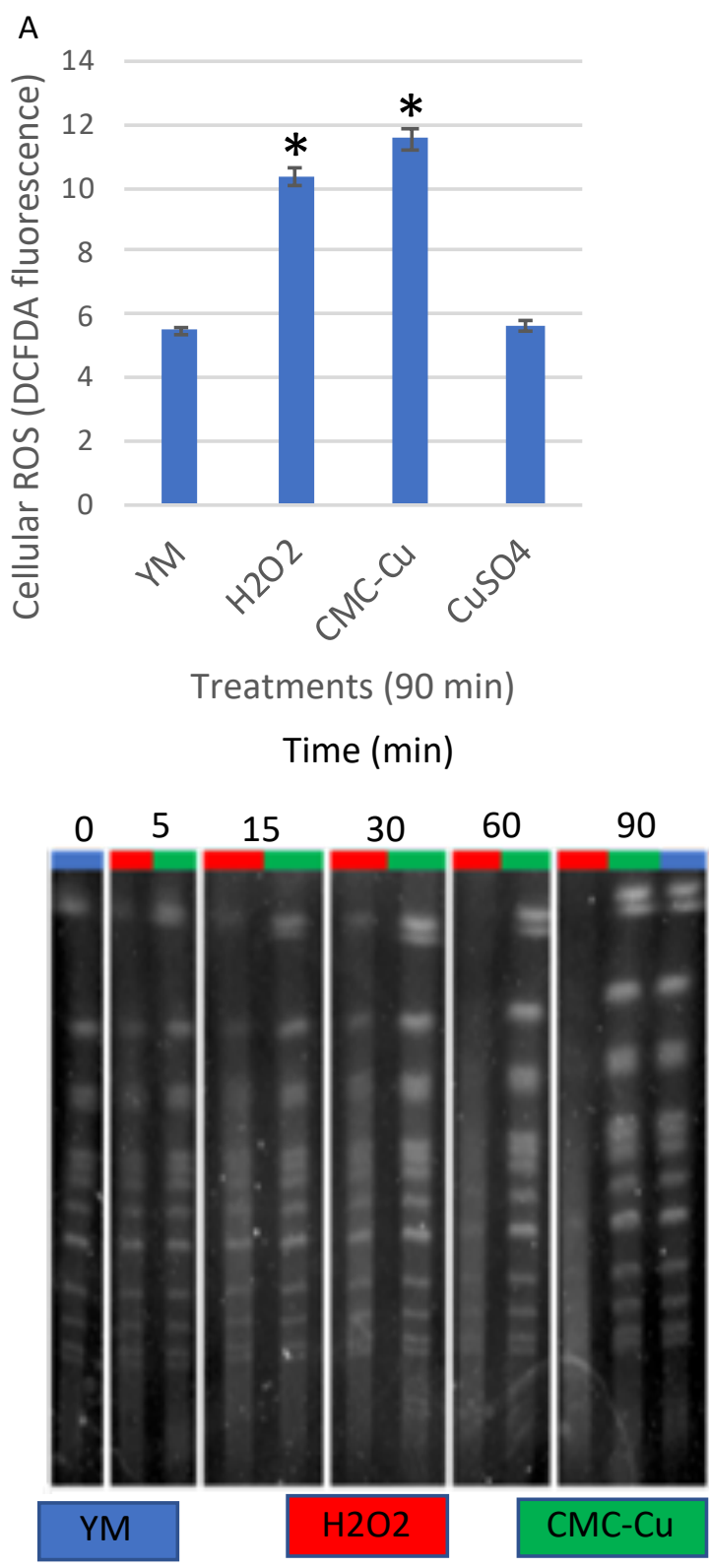

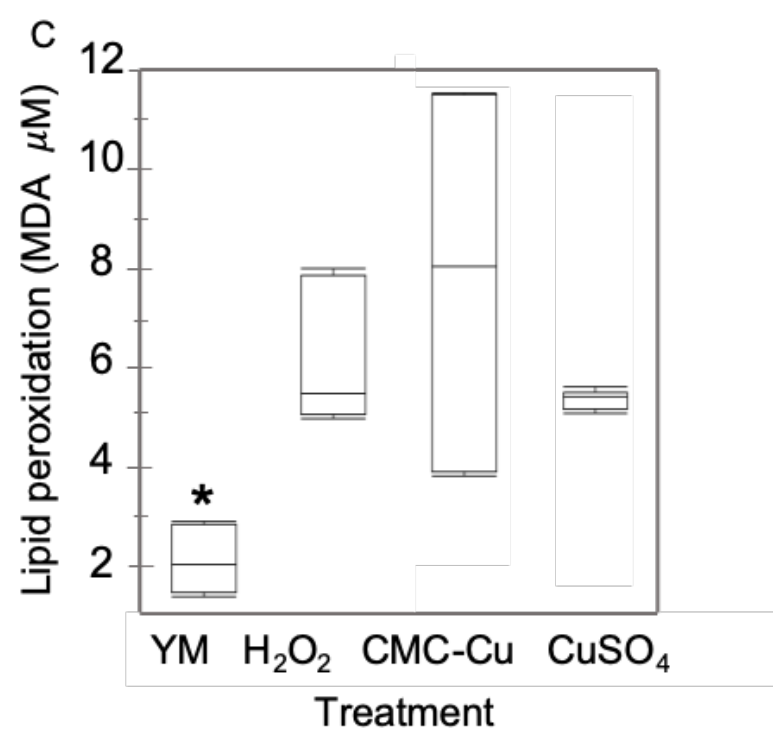

Figure 3-2. Cellular Toxicity Profiles Including ROS Generation and Biomolecule Damage. (A) ROS measurement by DCFDA fluorescence intensity showing $\mathrm{CMC}-\mathrm{Cu}$ and $\mathrm{H}_{2} \mathrm{O}_{2}$ producing higher amounts of $\mathrm{ROS}$ than $\mathrm{YM}$ or $\mathrm{CuSO}_{4}$. Statistical analysis performed via one-way ANOVA with Tukey HSD post-hoc analysis that suggested that $\mathrm{YM}$ and $\mathrm{CuSO}_{4}$ did not differ significantly while both $\mathrm{CMC}-\mathrm{Cu}$ and $\mathrm{H}_{2} \mathrm{O}_{2}$ were different from all other samples $(\mathrm{p}=0.05)$. (B) DNA damage qualitative assessment over time by PFGE comparing CMC-Cu treatment to $\mathrm{H}_{2} \mathrm{O}_{2}$ and cells grown in YM media. YM treatment is blue, $\mathrm{H}_{2} \mathrm{O}_{2}$ is red, and $\mathrm{CMC}-\mathrm{Cu}$ is green. $\mathrm{H}_{2} \mathrm{O}_{2}$ Chromosomal bands progressively get more ill-defined after 15 minutes while $\mathrm{CMC}-\mathrm{Cu}$ bands remain defined. (C) Lipid peroxidation measured by MDA production via TBARS assay after 90-minute treatment with $\mathrm{CMC}-\mathrm{Cu}$, $\mathrm{CuSO}_{4}, \mathrm{H}_{2} \mathrm{O}_{2}$, and cells grown in YM media. ANOVA statistical analysis was performed with Tukey-HSD post-hoc analysis and a p-value cut off of 0.05 . 
inducing a disulfide bond between cysteines of two glutathione molecules. While in yeast, $\mathrm{CuSO}_{4}$ toxicity is recused by both glutathione and precursor $\mathrm{N}$-acetylcysteine, but $\mathrm{CMC}-\mathrm{Cu}$ toxicity is only rescued by glutathione ${ }^{36}$. Several adaptations and resistance mechanisms in $S$. cerevisiae are known, of which the intracellular sequestration of $\mathrm{Cu}$ and the yeast strain resistance is largely predicted by the variation in copy number metallothionein Cup 1 . This copy number variation and resistance is seen in the type and sequence strain, S288 $\mathrm{c}^{94,95}$, from which the BY4741 strain used here is derived. This evidence, together with our findings on intracellular ROS suggesting that the reduction potential of $\mathrm{CMC}-\mathrm{Cu}$, contributes to its unique nanotoxicity.

\section{Genotoxicity}

In an approach to identify the primary process and kinetics of $\mathrm{CMC}-\mathrm{Cu}$ induced ROS damage to the yeast cells, the possibility of DNA damage was explored via a timed pulse-field gel electrophoresis (PFGE). As DNA is damaged, the accumulation of breaks causes the distinct chromosomal bands to be lost. The more ambiguous the chromosomal bands are in the PFGE, the more DNA damage has occurred. DNA damage by ROS has been well documented and implicated in mutagenesis and carcinogenesis ${ }^{96}$, but there remains some discussion about its role in the primary damaging process. Hydrogen peroxide and the superoxide anion damage DNA by creation of the hydroxyl radical and singlet oxygen which are energetic enough to have a direct effect on $\mathrm{DNA}^{97,98}$. CMC-Cu at $157 \mu \mathrm{M}$ caused little to no DNA damage over this study's typical 90-minute treatment when measured qualitatively by inspecting the chromosomal band integrity in the PFGE gel (Figure 3-2B). The chromosomal band integrity, band intensity, and background smearing of $\mathrm{CMC}-\mathrm{Cu}$ treated cells with those of the YM control were very similar in pattern. When treated with $489 \mu \mathrm{M}$ of $\mathrm{H}_{2} \mathrm{O}_{2}$ over time, a shift in the chromosomal band integrity was seen staring at the 15-minute mark and progressing until the 90-minute mark where all chromosomal band integrity was lost to DNA fragmentation represented as smearing across the gel. Hydrogen peroxide ${ }^{99}$ and copper at moderately toxic concentrations induce apoptosis in S. cerevisiae ${ }^{100}$. DNA fragmentation, phosphatidylserine externalization, and chromatin condensation are typical markers of yeast apoptosis ${ }^{101}$. Highly toxic levels of $\mathrm{Cu}$ induce necrosis from which the mitochondria has been implicated to play a role ${ }^{100}$. With these findings in sight, we hypothesized that CMC-Cu treatment under these conditions lacked DNA fragmentation but induced lipid damage to the cellular membrane damage.

\section{Lipid Peroxidation}

Aside from DNA, another target of cellular damage is the lipid membrane of the cell, and a compromised cellular membrane is a major action of antimicrobial copper. This investigation aimed to determine if lipid peroxidation was the primary process of CMC-Cu induced ROS damage to $S$. cerevisiae. ROS-induced lipid peroxidation ${ }^{102-104}$ and S. cerevisiae's cellular fatty acid composition-dependent susceptibility to copper ${ }^{9}$ was considered in forming this hypothesis. During lipid peroxidation, byproducts are formed of which malondialdehyde (MDA) is a convenient biomarker for lipid peroxidation ${ }^{105,106}$. Thiobarbituric acid (TBA) reacts with MDA forming a deeply colored chromogen fluorescently red adduct TBA-MDA ${ }^{107}$. By utilizing this reaction, we investigated lipid peroxidation by measuring the MDA-TBA adduct in a fluorescence assay known as a thiobarbituric acid reactive substances assay (TBARS). Dunnett's post-hoc statistical analysis found that the $\mathrm{CMC}-\mathrm{Cu}, \mathrm{CuSO}_{4}$, and $\mathrm{H}_{2} \mathrm{O}_{2}$ treatments all induced lipid peroxidation compared to the YM control media (Figure 3-2C). CMC-Cu treated cells analyzed via the TBARS assay produced the greatest fluorescence measuring at $8.03 \mu \mathrm{M} \mathrm{MDA}$ and within 
the biological replicates there was a wide-range. During our experiment we found a relatively large variation between our CMC-Cu exposures. This is likely related to particle size distribution and degree of agglomeration of the particles. Levels of lipid peroxidation caused by hydrogen peroxide and copper sulfate treated cells were very similar, measuring $5.60 \mu \mathrm{M}$ and $5.47 \mu \mathrm{M}$ MDA, respectively. These findings supported previous reports of copper's toxic interaction with cell membranes ${ }^{10,62}$. Our control sample in YM media measured 2.12 $\mu \mathrm{M}$ MDA. Unsaturated fatty acids are a major target of ROS that results in a cyclic reaction ${ }^{108,109}$ generating lipid radicals and toxic lipid hydroperoxides ${ }^{98,110}$. Hydroperoxides are unstable in the presence of metals and they are the primary product of lipid peroxidation. They attach to lipids such as free fatty acids, triacylglycerols, sterols, and phospholipids. In vivo decomposition of these compounds occurs via the reduction, in part enzymatically, by glutathione peroxidase typically using glutathione as a reductant ${ }^{107}$. This process ultimately leads to loss of membrane integrity and generation of aldehydes that can cause distant and localized lipid peroxidation ${ }^{111,112}$. The evidence that CMC$\mathrm{Cu}$ and $\mathrm{CuSO}_{4}$ initiated lipid peroxidation supports our hypothesis that the primary process of $\mathrm{CMC}-\mathrm{Cu}$ induced ROS damage to S. cerevisiae is lipid peroxidation.

\section{CMC-Cu Induced Alteration of Yeast Lipid Profile}

This study's results indicate that CMC-Cu induced ROS facilitated lipid peroxidation, but to further define the details of CMC-Cu nanotoxicity we employed an untargeted exploratory metabolomics assessment. By utilizing gas chromatography with electron spray ionization massspectroscopy (GC-ESI-MS) methodology the lipidomics aspect proved most interesting. Lipid extracts analyzed in the positive and negative modes of ESI-MS provided 610 potential features that were chiseled down to yield 105 tentative structure confidence level annotated compounds (Table S1). These were used as variables in the statistical analysis performed in MetaboAnalyst 4.0. We found that a partial least squared discriminant analysis (PLS-DA) technique was adequate in discriminating samples into their treatment groups of $\mathrm{CMC}-\mathrm{Cu}, \mathrm{CuSO}_{4}, \mathrm{CMC}$, and YM. A loadings matrix was made for the PLS-DA (Table S2). A PCA score plot, loading plot, and statistical ANOVA analysis was produced (Figure 3-S2), but the PCA did not provide as good of separation as the PLS-DA. Using three components, we found a clear separation between all of the treatment groups. By using 2 components, 1 and 2, the PLS-DA had an overlap of the YM control and CMC-Cu treatment (Figure 3-3A). With components 1 and 3, the PLS-DA had an overlap of CMC-Cu and $\mathrm{CuSO}_{4}$ treatments (Figure 3-3B). A permutation test was administered that showed the supervised method did not overfit the data, enabling interpretation of the results, $\mathrm{p}<0.001$. This result is suggestive of the similarity between soluble copper toxicity but also supportive of the unique nature of the CMC-Cu nanotoxicity. Component 1 explained $17.8 \%$ of the variance, component 2 explained $15.7 \%$, and component 3 explained $27.7 \%$ of the variance between samples. The heatmap (Figure 3-3C) of the top 10 compounds averaged per treatment groups showed the cellular membrane phospholipids phosphatidylethanolamine (PE) and phosphatidylcholine (PC) increased in relative abundance for $\mathrm{CMC}-\mathrm{Cu}$ treatments. A whole lipidome heatmap was also generated (Figure 3-S3). Variable importance in Projection score plot was generated to give insight to potential biomarkers for $\mathrm{CMC}-\mathrm{Cu}$ induced toxicity (Figure 2-S4). In vitro studies have shown that copper ions interact with synthetic membrane models in an ordered fashion first with PC, then PE based on the structure of the membrane prevalent phospholipids ${ }^{10}$. We found that PC and PE composition responded to $\mathrm{CMC}-\mathrm{Cu}$ and $\mathrm{CuSO}_{4}$ exposure, albeit in opposing fashion. Unexpectedly, we found that $\mathrm{CMC}$ had a depleting effect on lipid composition. PC and PE are the chief membrane lipid 
components from which their composition influences physico-chemical properties altering basic biological function in yeast such as cell growth, budding, membrane trafficking, and other formations ${ }^{113-115}$.

The biosynthesis of fatty acids/ acyl chains starts with the conversion of acetyl-CoA into malonylCoA from which the acetyl chain develops mainly C16:0 and C18:0 before desaturation in the endoplasmic reticulum (ER) of S. cerevisiae ${ }^{116}$. This acyl-CoA pool can be then converted into bulk phospholipids through various pathways involving the ER and mitochondria ${ }^{113}$. The Kennedy pathway allows PE and PC formation from acyl-CoA and triacylglycerols (TG) pools. This study found that CMC-Cu treated cells depleted TG reserves (Figure 3-S3) and increased important PE and PC levels (Figure 3-3), suggesting a disruption in lipid homeostasis. TGs stored in lipid droplets protect lipid homeostasis by balancing fatty acid saturation ${ }^{117}$ and serving as a base for phospholipid metabolism ${ }^{118}$. When needed, TGs are hydrolyzed to provide building blocks for membrane lipid synthesis, delivering a quick response to changing environmental conditions ${ }^{119}$. TG can be converted to PC and PE through the Kennedy pathway ${ }^{113,119}$. These results support the hypothesis that $\mathrm{CMC}-\mathrm{Cu}$ treated cells are depleted of their protective TG content through an increase in PC and PE membrane production.
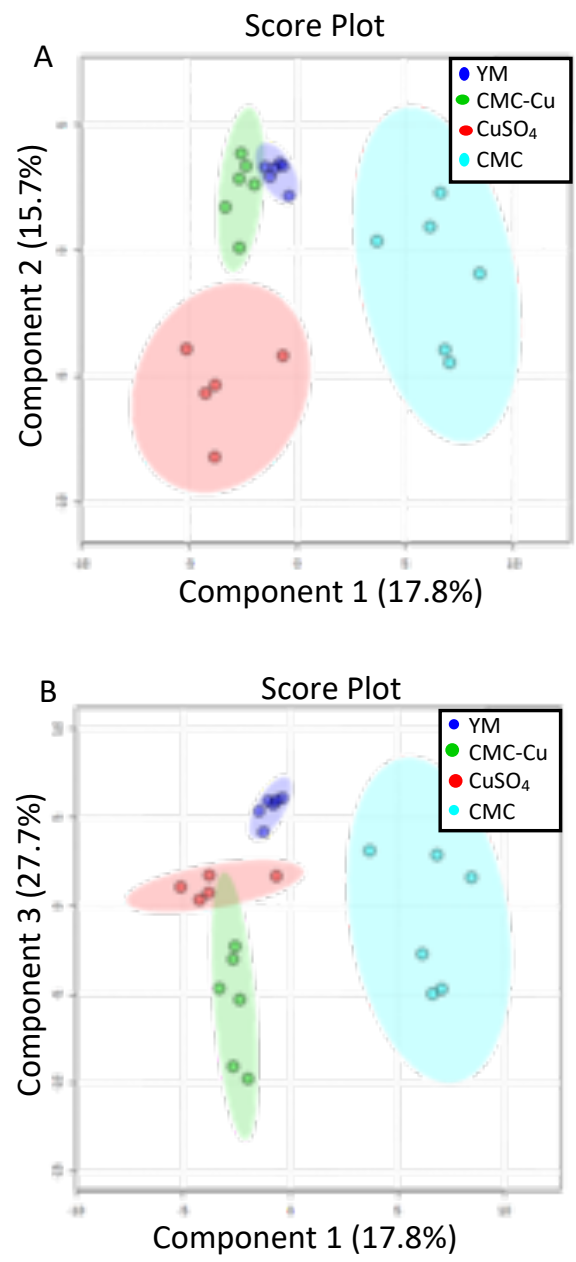

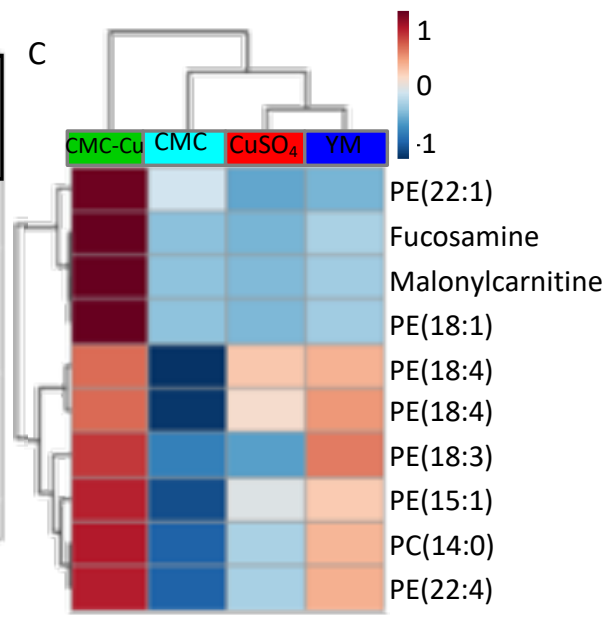

Figure 3-3. Lipid Metabolic Profiling in Response to $\mathrm{CMC}-\mathrm{Cu}$ Treatment. (A) Partial least squares discriminant analysis (PLS-DA) showing component 1 and component 2 separated all treatments but YM and CMC-Cu. (B) PLS-DA showing component 1 and component 3 separated all treatments but CMC-Cu and $\mathrm{CuSO}_{4}$ as well as YM and CMC-Cu. Taken together, components 1,2 , and 3 provide a 3 -dimensional separation of all treatments based on their lipid profiles. (C) Heatmap of the top 10 metabolites used in profiling yeast treatment groups. Samples are divided into columns, compounds are divided by rows, and the color is indicative of relative abundance. 
Interestingly, in $\mathrm{CMC}-\mathrm{Cu}$ treated yeast we found relatively high malonylcarnitine levels, a metabolite involved with the transportation of long-chain fatty acids into the mitochondria (Figure 3-3C). Increased levels of malonylcarnitine are indicative of fatty acid oxidation disruption and mitochondrial respiratory chain failure ${ }^{120-124}$. Cellular copper is distributed throughout the entire cell and a pool of bioavailable copper is also present within the mitochondria ${ }^{125}$, enabling mitochondrial metallation reactions. Formation of the $\mathrm{Cu}$ centers bound within cytochrome $\mathrm{c}$ oxidase $(\mathrm{CcO})$ occurs in the mitochondrial inner membrane space (IMS) during which two copperbinding subunits of $\mathrm{CcO}$ become metallated ${ }^{21}$. The detection of relatively high levels of malonylcarnitine further suggests that lipid damage is a primary target of the yeast cell damage, as this metabolite is indictive of fatty chain transportation disruption. Additionally, the amino-sugar fucosamine also increased in relative abundance during $\mathrm{CMC}-\mathrm{Cu}$ treatment. Other amino-sugars such as fructosamine have been shown to complex with redox-active metals, chelating copper, iron, and nickel metals displaying some antioxidant aspects ${ }^{126-128}$, although the research on fucosamine is still in preliminary stages. Primary metabolites were also extracted and analyzed by GC-MS techniques but changes in the metabolism could not be used in a discriminating analysis without overfitting the data (data not shown). Together this lipid profiling and TBARS assay support lipid damage as a primary target of $\mathrm{CMC}-\mathrm{Cu}$ induced nanotoxicity.
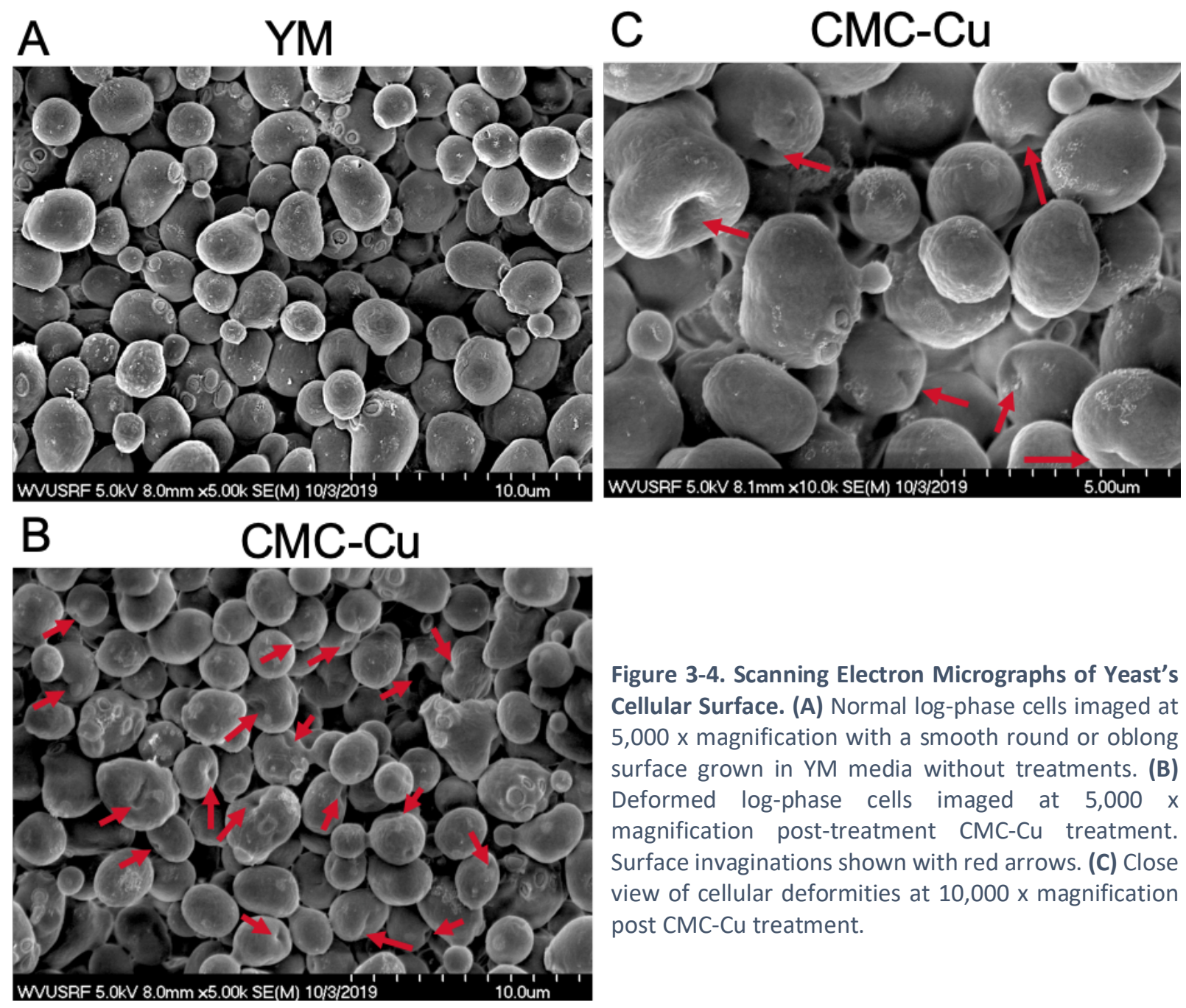

Figure 3-4. Scanning Electron Micrographs of Yeast's Cellular Surface. (A) Normal log-phase cells imaged at $5,000 \times$ magnification with a smooth round or oblong surface grown in YM media without treatments. (B) Deformed log-phase cells imaged at 5,000 $x$ magnification post-treatment $\mathrm{CMC}-\mathrm{Cu}$ treatment. Surface invaginations shown with red arrows. (C) Close view of cellular deformities at 10,000 x magnification post CMC-Cu treatment. 


\section{Membrane Bending Induced by CMC-Cu Exposure}

As the major phospholipid bilayer components $\mathrm{PC}$ and $\mathrm{PE}$ change in response to $\mathrm{CMC}-\mathrm{Cu}$ exposure, we focused on imaging to this cellular damage in high-resolution detail by employing scanning electron microscopy (SEM) to visualize cellular surface damage. With the use of SEM, we were able to visualize the cellular surface prior to treatment and post-CMC-Cu. We found deformities of the cell surface resembling invaginations in cells treated with $\mathrm{CMC}-\mathrm{Cu}$ compared to cells grown in YM medium (Figure 3-4). Log-phase cells grown in YM media were circular/ oval, smooth, and round in appearance (Figure 3-4A). The log phase cells were grown in YM with $157 \mu \mathrm{M} \mathrm{CMC}-\mathrm{Cu}$ had surface deformations that are distinguished by red arrows (Figure 34B). Two images were chosen to be representative of treated and untreated population differences at 5,000 x magnification displaying the cellular surfaces of approximately 100 cells. At a higher 10,000 x magnification, we found varying degrees of cell surface invaginations on both mother and daughter cells (Figure 3-4C). Ethanol treatment has been shown to produce a similar abnormal cell surface phenotype in yeast when visualized by electron micrographs ${ }^{129}$. These major alterations to the cellular microstructure have previously been used as a marker in characterizing novel antimicrobial treatments ${ }^{130,131}$. These abnormal morphologies of the cellular microstructure are indicative of cell surface damage. Prior research concerning the location of $\mathrm{CMC}-\mathrm{Cu}$ stained with fluorescein isothiocyanate (FITC) suggested an association with the cellular surface for both $\mathrm{CMC}-\mathrm{Cu}$ and $\mathrm{CMC}$ treatments ${ }^{36}$. Findings from the SEM images further support cell surface damage owing to membrane lipid peroxidation as the primary process by which $\mathrm{CMC}-\mathrm{Cu}$ exerts its toxicity.

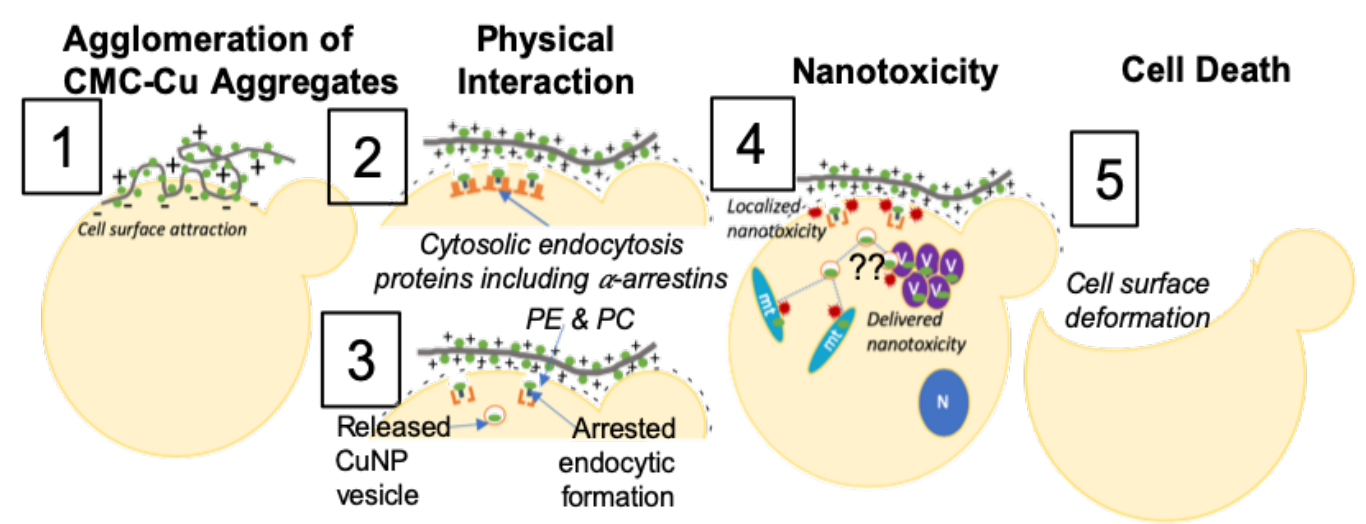

Attempted Endocytosis

Figure 3-5. Model of CMC-Cu Toxicity. Carboxymethyl cellulose copper nanoparticles are attracted to the cells surface due to charge in stage 1. Yeast cells recognize copper inducing the $\alpha$ arrestin mediated endocytosis during stage 2. Because of the copper nanoparticles' stable attachment to the CMC fibrils, a subset of CuNPs stays on the cell's surface causing localized damage to the phospholipids of the plasma membrane while another subset causes sub-cellular damage due to surface area toxicity in stage $3 \&$ stage 4 . The consequence of interaction with $\mathrm{CMC}-\mathrm{Cu}$ results in a deformation of the cell's surface, as seen in stage $\mathbf{5}$.

\section{Conclusions}

While performing a nanotoxicological investigation of the copper nanoparticles attached to the cellulose derivative $\mathrm{CMC}$, we uncovered several results worth noting. The metal homeostasis 
between zinc and copper becomes unbalanced in sensitive yeast strains upon treatment with CMC$\mathrm{Cu}$. Exposure of this substance to $S$. cerevisiae produce ROS that did not indicate DNA damage but did suggest lipid peroxidation. Further exploration revealed $\mathrm{CMC}-\mathrm{Cu}$ altered major membrane phospholipids and depleted TG lipids that act to balance fatty acid saturation. Moreover, we visualized cell surface damage in great detail, supporting that the primary mechanism of action of $\mathrm{CMC}-\mathrm{Cu}$ exposure is surface membrane damage. Secondary processes are involved in this nanotoxicity, and in addition to the metal homeostasis, $\alpha$-arrestins Aly1 and Aly2 have been suggested to facilitate endocytosis of the copper nanoparticles. A model was created to visually convey $\mathrm{CMC}-\mathrm{Cu}$-induced nanotoxicological damage based on experimental findings thus far (Figure 3-5). During the first of five transient stages, $\mathrm{CMC}-\mathrm{Cu}$ aggregate on the cellular surface likely owing to the positive charge of the cell wall. Secondarily, cell surface attachments physically interact with these $\mathrm{CMC}-\mathrm{Cu}$. There may be an attempt to endocytose $\mathrm{CMC}-\mathrm{Cu}$, which is likely to incompletely occur if the CuNPs are still reduced on CMC or likely occurs if CuNPs are free from the cellulose structure. The mode of $\mathrm{CMC}-\mathrm{Cu}$ nanotoxicity to $S$. cerevisiae occurs via localized damage primarily to the cellular membrane with secondary damages occurring via disruption of metal homeostasis and disruption of cellular functions likely in or near the vacuole and mitochondria. A deformed and invaginated cellular surface is the result of $\mathrm{CMC}-\mathrm{Cu}$ exposure found in this study. There are many overlaps in the toxicity of copper and the nanotoxicity of copper nanoparticles discussed earlier, but as nanotechnology becomes more ubiquitous the need for understanding these materials grow.

Acknowledgments: MJW was supported by the Nation Science Foundation's Integrative Graduate Education and Research Traineeship: Research and Education in Nanotoxicology at West Virginia University (IGERT:REN@WVU) award number 1144676. We acknowledge Amaury Pupo Meriño for help with the GC-ESI/MS study and analysis, Marcela Redigolo for help using the electron microscope, Dr. Jonathan Cumming for help with the ICP-OES, Dr. Gloria Oporto for generously supplying the nanoparticles, Dr. Kang Mo $\mathrm{Ku}$ for help with the metabolomics studies, and The WVU Genomics Corps for use of their instruments. 


\section{References}

1. Gabbay, J. et al. Copper oxide impregnated textiles with potent biocidal activities. J. Ind. Text. 35, 323-335 (2006).

2. Palza, H. Antimicrobial polymers with metal nanoparticles. Int. J. Mol. Sci. 16, 2099-2116 (2015).

3. Cioffi, N. et al. Copper nanoparticle/polymer composites with antifungal and bacteriostatic properties. Chem. Mater. 17, 5255-5262 (2005).

4. Borkow, G. \& Gabbay, J. Copper, An Ancient Remedy Returning to Fight Microbial, Fungal and Viral Infections. Curr. Chem. Biol. 3, 272-278 (2009).

5. Hostynek, J. J. \& Maibach, H. I. Copper hypersensitivity: dermatologic aspects. Dermatol. Ther. 17, 328-333 (2004).

6. Lemire, J. A., Harrison, J. J. \& Turner, R. J. Antimicrobial activity of metals: Mechanisms, molecular targets and applications. Nat. Rev. Microbiol. 11, 371-384 (2013).

7. Finney, L. A. \& O'Halloran, T. V. Transition metal speciation in the cell: Insights from the chemistry of metal ion receptors. Science (80-90. ). 300, 931-936 (2003).

8. Zhang, J. et al. ROS and ROS-Mediated Cellular Signaling. Oxid. Med. Cell. Longev. 2016, 1-18 (2016).

9. Avery, S., Howlett, N. \& Radice, S. Copper toxicity towards Saccharomyces cerevisiae: dependence on plasma membrane fatty acid composition. Appl. Envir. Microbiol. 62, 39603966 (1996).

10. Suwalsky, M., Ungerer, B., Quevedo, L., Aguilar, F. \& Sotomayor, C. P. Cu2+ ions interact with cell membranes. J. Inorg. Biochem. 70, 233-238 (1998).

11. Allen, H. E., Brisbin, T. D. \& Hall, R. H. Metal Speciation. Effects on Aquatic Toxicity. Environ. Sci. Technol. 14, 441-443 (1980).

12. Allen, H. E. \& Hansen, D. J. The importance of trace metal speciation to water quality criteria. Water Environ. Res. 68, 42-54 (1996).

13. Haas, K. L. \& Franz, K. J. Application of Metal Coordination Chemistry to Explore and Manipulate Cell Biology. Chem. Rev. 109, 4921-4960 (2009).

14. Ma, Z., Jacobson, F. E. \& Giedroc, D. P. Metal Transporters and Metal Sensors: How Coordination Chemistry Controls Bacterial Metal Homeostasis. October 109, 4644-4681 (2009).

15. Waldron, K. J., Rutherford, J. C., Ford, D. \& Robinson, N. J. Metalloproteins and metal sensing. Nature 460, 823-830 (2009).

16. Irving, H. \& Williams, R. J. P. The Stability of Transition-metal Complexes. J. Chem. Soc 3192-3210 (1953).

17. Waldron, K. J. \& Robinson, N. J. How do bacterial cells ensure that metalloproteins get the correct metal? Nat. Rev. Microbiol. 7, 25-35 (2009).

18. Andreini, C., Bertini, I. \& Rosato, A. A hint to search for metalloproteins in gene banks. Bioinformatics 20, 1373-1380 (2004).

19. Wang, Y. et al. The cellular economy of the Saccharomyces cerevisiae zinc proteome. metallomics 10, 1755-1776 (2018).

20. Kim, B. E., Nevitt, T. \& Thiele, D. J. Mechanisms for copper acquisition, distribution and regulation. Nat. Chem. Biol. 4, 176-185 (2008).

21. Leary, S. C., Winge, D. R. \& Cobine, P. A. "Pulling the plug" on cellular copper: The role of mitochondria in copper export. Biochim. Biophys. Acta-Mol. Cell Res. 1793, 146-153 
(2009).

22. Portnoy, M. E., Schmidt, P. J., Rogers, R. S. \& Culotta, V. C. Metal transporters that contribute copper to metallochaperones in Saccharomyces cerevisiae. Mol. Genet. Genomics 265, 873-882 (2001).

23. Hassett, R., Dix, D. R., Eide, D. J. \& Kosman, D. J. The Fe(II) permease Fet4p functions as a low affinity copper transporter and supports normal copper trafficking in Saccharomyces cerevisiae. Biochem. J. 351, 477-484 (2000).

24. Huffman, D. L. \& O'Halloran, T. V. Function, Structure, and Mechanism of Interacellular Copper Trafficking Proteins. Annu. Rev. Biochem. 70, 677-701 (2001).

25. Banci, L. et al. The Atx1-Ccc2 complex is a metal-mediated protein-protein interaction. Nat. Chem. Biol. 2, 367-368 (2006).

26. O'Halloran, T. V. \& Culotta, V. C. Metallochaperones, an intracellular shuttle service for metal ions. Journal of Biological Chemistry 275, 25057-25060 (2000).

27. Furukawa, Y., Torres, A. S. \& O'Halloran, T. V. Oxygen-induced maturation of SOD1: A key role for disulfide formation by the copper chaperone CCS. EMBO J. 23, 2872-2881 (2004).

28. Lin, S. J., Pufahl, R. A., Dancis, A., O'Halloran, T. V. \& Culotta, V. C. A role for the Saccharomyces cerevisiae ATX1 gene in copper trafficking and iron transport. J. Biol. Chem. 272, 9215-9220 (1997).

29. Yuan, D. S. et al. The Menkes/Wilson disease gene homologue in yeast provides copper to a ceruloplasmin-like oxidase required for iron uptake. Proc. Natl. Acad. Sci. U. S. A. 92, 2632-2636 (1995).

30. Jungmann, J. et al. $\mathrm{MAC1}$, a nuclear regulatory protein related to $\mathrm{Cu}$-dependent transcription factors is involved in $\mathrm{Cu} / \mathrm{Fe}$ utilization and stress resistance in yeast. EMBO J. 12, 5051-5056 (1993).

31. Labbé, S., Zhu, Z. \& Thiele, D. J. Copper-specific transcriptional repression of yeast genes encoding critical components in the copper transport pathway. J. Biol. Chem. 272, 1595115958 (1997).

32. Thiele, D. J. ACE1 regulates expression of the Saccharomyces cerevisiae metallothionein gene. Mol. Cell. Biol. 8, 2745-2752 (1988).

33. Gralla, E. B., Thiele, D. J., Silar, P. \& Valentine, J. S. ACE1, a copper-dependent transcription factor, activates expression of the yeast copper, zinc superoxide dismutase gene. Proc. Natl. Acad. Sci. U. S. A. 88, 8558-8562 (1991).

34. Culotta, V. C., Howard, W. R. \& Liu, X. F. CRS5 encodes a metallothionein-like protein in Saccharomyces cerevisiae. J. Biol. Chem. 269, 25295-25302 (1994).

35. Tamai, K. T., Gralla, E. B., Ellerby, L. M., Valentine, J. S. \& Thiele, D. J. Yeast and mammalian metallothioneins functionally substitute for yeast copper-zinc superoxide dismutase. Proc. Natl. Acad. Sci. U. S. A. 90, 8013-8017 (1993).

36. Rong-Mullins, X. et al. Proteomic and genetic analysis of the response of S. cerevisiae to soluble copper leads to improvement of the antimicrobial function of cellulosic copper nanoparticles. Metallomics 9, 1304-1315 (2017).

37. Fogel, S. \& Welch, J. W. Tandem gene amplification mediates copper resistance in yeast. Proc. Natl. Acad. Sci. U. S. A. 79, 5342-5346 (1982).

38. Grass, G. et al. The Metal Permease ZupT from. Society 187, 1604-1611 (2005).

39. Lin, W., Chai, J., Love, J. \& Fu, D. Selective electrodiffusion of zinc ions in a Zrt-, Irt-like protein, ZIPB. J. Biol. Chem. 285, 39013-39020 (2010). 
40. Jensen, L. T., Ajua-Alemanji, M. \& Culotta, V. C. The Saccharomyces cerevisiae High Affinity Phosphate Transporter Encoded by PHO84 Also Functions in Manganese Homeostasis. J. Biol. Chem. 278, 42036-42040 (2003).

41. MacDiarmid, C. W., Gaither, L. A. \& Eide, D. Zinc transporters that regulate vacuolar zinc storage in Saccharomyces cerevisiae. EMBO J. 19, 2845-2855 (2000).

42. Li, L., Chen, O. S., Ward, D. M. V. \& Kaplan, J. CCC1 Is a Transporter That Mediates Vacuolar Iron Storage in Yeast. J. Biol. Chem. 276, 29515-29519 (2001).

43. Habibi, Y., Lucia, L. A. \& Rojas, O. J. Cellulose nanocrystals: Chemistry, self-assembly, and applications. Chem. Rev. 110, 3479-3500 (2010).

44. Anderson, R. L., Owens, J. W. \& Timms, C. W. The toxicity of purified cellulose in studies with laboratory animals. Cancer Lett. 63, 83-92 (1992).

45. Cullen, R. T., Miller, B. G., Jones, A. D. \& Davis, J. M. G. Toxicity of cellulose fibres. Ann. Occup. Hyg. 46, 81-84 (2002).

46. Owens, C. W., Oporto, G. S., Söderberg, B. C. G. \& Lambson, K. E. Lignocellulosic Microand Nanomaterials as Copper Frames for the Evaluation of the Copper(I)-Catalyzed AzideAlkyne Cycloaddition. J. Nanomater. 2017, (2017).

47. Zhong, T. et al. Antimicrobial Properties of the Hybrid Copper NanoparticlesCarboxymethyl Cellulose. Wood Fiber Sci. 45, 215-222 (2013).

48. Hassanzadeh, M. et al. Nanofibrillated Cellulose from Appalachian Hardwoods Logging Residues as Template for Antimicrobial Copper. J. Nanomater. 2017, (2017).

49. Winans, M. J., Gallagher, J. E. G., Jaczynski, J. \& Oporto, G. Pick Your Poison: Benzalkonium Chloride and Copper Enable Nanocellulose Derivatives to Form Antimicrobial Properties Against a Spectrum of Microorganisms. bioRxiv 783076 (2019). doi:10.1101/783076

50. Zhong, T., Oporto, G. S., Jaczynski, J. \& Jiang, C. Nanofibrillated Cellulose and Copper Nanoparticles Embedded in Polyvinyl Alcohol Films for Antimicrobial Applications. Biomed Res. Int. 2015, 1-8 (2015).

51. Jiang, C., Oporto, G. S., Zhong, T. \& Jaczynski, J. TEMPO nanofibrillated cellulose as template for controlled release of antimicrobial copper from PVA films. Cellulose 23, 713722 (2016).

52. Kasemets, K., Ivask, A., Dubourguier, H. C. \& Kahru, A. Toxicity of nanoparticles of ZnO, $\mathrm{CuO}$ and $\mathrm{TiO} 2$ to yeast Saccharomyces cerevisiae. Toxicol. Vitr. 23, 1116-1122 (2009).

53. Karlsson, H. L., Cronholm, P., Gustafsson, J. \& Möller, L. Copper oxide nanoparticles are highly toxic: A comparison between metal oxide nanoparticles and carbon nanotubes. Chem. Res. Toxicol. 21, 1726-1732 (2008).

54. Midander, K. et al. Surface characteristics, copper release, and toxicity of nano- and micrometer-sized copper and copper(11) oxide particles: A cross-disciplinary study. Small 5, 389-399 (2009).

55. Baatrup, E. Structural and functional effects of heavy metals on the nervous system, including sense organs, of fish. Comp. Biochem. Physiol. Part C, Comp. 100, 253-257 (1991).

56. Duina, A. A., Miller, M. E. \& Keeney, J. B. Budding yeast for budding geneticists: A primer on the Saccharomyces cerevisiae model system. Genetics 197, 33-48 (2014).

57. Kukulski, W., Schorb, M., Kaksonen, M. \& Briggs, J. A. G. Plasma membrane reshaping during endocytosis is revealed by time-resolved electron tomography. Cell 150, 508-520 (2012). 
58. Nikko, E. \& Pelham, H. R. B. Arrestin-mediated endocytosis of yeast plasma membrane transporters. Traffic 10, 1856-1867 (2009).

59. Nikko, E., Sullivan, J. A. \& Pelham, H. R. B. Arrestin-like proteins mediate ubiquitination and endocytosis of the yeast metal transporter Smf1. EMBO Rep. 9, 1216-1221 (2008).

60. Lin, C. H., MacGurn, J. A., Chu, T., Stefan, C. J. \& Emr, S. D. Arrestin-Related UbiquitinLigase Adaptors Regulate Endocytosis and Protein Turnover at the Cell Surface. Cell 135, 714-725 (2008).

61. Prosser, D. C. et al. Alpha-Arrestins participate in cargo selection for both clathrinindependent and clathrin-mediated endocytosis. J. Cell Sci. 128, 4220-4234 (2015).

62. Avery, S. V. S., Howlett, N. G. N. \& Radice, S. Copper toxicity towards Saccharomyces cerevisiae: Dependence on plasma membrane fatty acid composition. Appl. Environ. Microbiol. 62, 3960-3966 (1996).

63. Letelier, M. E. et al. Possible mechanisms underlying copper-induced damage in biological membranes leading to cellular toxicity. Chem. Biol. Interact. 151, 71-82 (2005).

64. Pupo, A. et al. MCHM Acts as a Hydrotrope, Altering the Balance of Metals in Yeast. Biol. Trace Elem. Res. 1-12 (2019). doi:10.1007/s12011-019-01850-z

65. Kobashigawa, L. C., Xu, Y. C., Padbury, J. F., Tseng, Y. T. \& Yano, N. Metformin protects cardiomyocyte from doxorubicin induced cytotoxicity through an AMP-activated protein kinase dependent signaling pathway: An in Vitro study. PLoS One 9, (2014).

66. Bourque, S. D. \& Titorenko, V. I. A quantitative assessment of the yeast lipidome using electrospray ionization mass spectrometry. J. Vis. Exp. 1-3 (2009). doi:10.3791/1513

67. Pupo, A., Ku, K. M. \& Gallagher, J. Effects of MCHM on yeast metabolism. PLoS One 14, e0223909 (2019).

68. Smith, C. A., Want, E. J., O’Maille, G., Abagyan, R. \& Siuzdak, G. XCMS: Processing mass spectrometry data for metabolite profiling using nonlinear peak alignment, matching, and identification. Anal. Chem. 78, 779-787 (2006).

69. Du, P., Kibbe, W. A. \& Lin, S. M. Improved peak detection in mass spectrum by incorporating continuous wavelet transform-based pattern matching. Bioinformatics 22, 2059-2065 (2006).

70. Zhou, B., Wang, J. \& Ressom, H. W. Metabosearch: Tool for mass-based metabolite identification using multiple databases. PLoS One 7, (2012).

71. Wishart, D. S. et al. HMDB 4.0: The human metabolome database for 2018. Nucleic Acids Res. 46, D608-D617 (2018).

72. Guijas, C. et al. METLIN: A Technology Platform for Identifying Knowns and Unknowns. Physiol. Behav. 5, 3156-3164 (2018).

73. Cui, Q. et al. Metabolite identification via the Madison Metabolomics Consortium Database. Nat. Biotechnol. 26, 162-164 (2008).

74. Fahy, E., Sud, M., Cotter, D. \& Subramaniam, S. LIPID MAPS online tools for lipid research. Nucleic Acids Res. 35, 606-612 (2007).

75. Ramirez-Gaona, M. et al. YMDB 2.0: A significantly expanded version of the yeast metabolome database. Nucleic Acids Res. 45, D440-D445 (2017).

76. Kim, S. et al. PubChem 2019 update: Improved access to chemical data. Nucleic Acids Res. 47, D1102-D1109 (2019).

77. Xia, J., Sinelnikov, I. V., Han, B. \& Wishart, D. S. MetaboAnalyst 3.0-making metabolomics more meaningful. Nucleic Acids Res. 43, W251-W257 (2015).

78. Eide, D. J. Zinc transporters and the cellular trafficking of zinc. Biochim. Biophys. Acta - 
Mol. Cell Res. 1763, 711-722 (2006).

79. Fierke, C. A. \& Thompson, R. B. Fluorescence-based biosensing of zinc using carbonic anhydrase. BioMetals 14, 205-222 (2001).

80. Vinkenborg, J. L., Koay, M. S. \& Merkx, M. Fluorescent imaging of transition metal homeostasis using genetically encoded sensors. Curr. Opin. Chem. Biol. 14, 231-237 (2010).

81. Dittmer, P. J., Miranda, J. G., Gorski, J. A. \& Palmer, A. E. Genetically encoded sensors to elucidate spatial distribution of cellular zinc. J. Biol. Chem. 284, 16289-16297 (2009).

82. Devirgiliis, C., Murgia, C., Danscher, G. \& Perozzi, G. Exchangeable zinc ions transiently accumulate in a vesicular compartment in the yeast Saccharomyces cerevisiae. Biochem. Biophys. Res. Commun. 323, 58-64 (2004).

83. Krężel, A. \& Maret, W. The functions of metamorphic metallothioneins in zinc and copper metabolism. Int. J. Mol. Sci. 18, 1-20 (2017).

84. MacDiarmid, C. W. Zinc transporters that regulate vacuolar zinc storage in Saccharomyces cerevisiae. EMBO J. 19, 2845-2855 (2000).

85. Clarkson, T. W. Molecular and Ionic Mimicry of Toxic Metals. Annu. Rev. Pharmacol. Toxicol. 545-571 (1993).

86. Stohs, S. J. \& Bagchi, D. Oxidative mechanisms in the toxicity of metal ions. Free Radic. Biol. Med. 18, 321-336 (1995).

87. Valko, M., Morris, H. \& Cronin, M. T. D. Metals, Toxicity and Oxidative Stress. Curr. Med. Chem. 12, 1161-1208 (2005).

88. Macomber, L. \& Imlay, J. A. The iron-sulfur clusters of dehydratases are primary intracellular targets of copper toxicity. Proc. Natl. Acad. Sci. U. S. A. 106, 8344-8349 (2009).

89. Xu, F. F. \& Imlay, J. A. Silver(I), mercury(II), cadmium(II), and zinc(II) target exposed enzymic iron-sulfur clusters when they toxify Escherichia coli. Appl. Environ. Microbiol. 78, 3614-3621 (2012).

90. Valko, M., Rhodes, C. J., Moncol, J., Izakovic, M. \& Mazur, M. Free radicals, metals and antioxidants in oxidative stress-induced cancer. Chemico-Biological Interactions 160, 1-40 (2006).

91. Fauchon, M. et al. Sulfur sparing in the yeast proteome in response to sulfur demand. Mol. Cell 9, 713-723 (2002).

92. Helbig, K., Grosse, C. \& Nies, D. H. Cadmium toxicity in glutathione mutants of Escherichia coli. J. Bacteriol. 190, 5439-5454 (2008).

93. Helbig, K., Bleuel, C., Krauss, G. J. \& Nies, D. H. Glutathione and transition-metal homeostasis in Escherichia coli. J. Bacteriol. 190, 5431-5438 (2008).

94. Zhao, Y. et al. Structures of naturally evolved CUP1 tandem arrays in yeast indicate that these arrays are generated by unequal nonhomologous recombination. G3 Genes, Genomes, Genet. 4, 2259-2269 (2014).

95. Karin, M. et al. Primary structure and transcription of an amplified genetic locus: The CUP1 locus of yeast. Proc. Natl. Acad. Sci. U. S. A. 81, 337-341 (1984).

96. Ames, B. N. \& Gold, L. S. Endogenous mutagens and the causes of aging and cancer. Mutat. Res. - Fundam. Mol. Mech. Mutagen. 250, 3-16 (1991).

97. Halliwell, B. \& Aruoma, O. I. DNA damage by oxygen-derived species. Fed. Eur. Biochem. Soc. 281, 9-19 (1991).

98. Perrone, G. G., Tan, S. X. \& Dawes, I. W. Reactive oxygen species and yeast apoptosis. 
Biochim. Biophys. Acta - Mol. Cell Res. 1783, 1354-1368 (2008).

99. Madeo, F. et al. Oxygen Stress : A Regulator of Apoptosis in Yeast. 145, 757-767 (1999).

100. Liang, Q. \& Zhou, B. Copper and manganese induce yeast apoptosis via different pathways. Mol. Biol. Cell 18, 4741-4749 (2007).

101. Madeo, F., Fröhlich, E. \& Fröhlich, K. U. A yeast mutant showing diagnostic markers of early and late apoptosis. J. Cell Biol. 139, 729-734 (1997).

102. Reekmans, R., De Smet, K., Chen, C., Van Hummelen, P. \& Contreras, R. Old yellow enzyme interferes with Bax-induced NADPH loss and lipid peroxidation in yeast. FEMS Yeast Res. 5, 711-725 (2005).

103. Jian, W., Arora, J. S., Oe, T., Shuvaev, V. V. \& Blair, I. A. Induction of endothelial cell apoptosis by lipid hydroperoxide-derived bifunctional electrophiles. Free Radic. Biol. Med. 39, 1162-1176 (2005).

104. Zhivotovsky, B. \& Kroemer, G. Apoptosis and genomic instability. Nat. Rev. Mol. Cell Biol. 5, 752-762 (2004).

105. Pryor, W. A. On the detection of lipid hydroperoxides in biological samples. Free Radic. Biol. Med. 7, 177-178 (1989).

106. Esterbauer, H. \& Cheeseman, K. H. Determination of Aldehydic Lipid Peroxidation Products: Malonaldehyde and 4-Hydroxynonenal. Methods Enzymol. 186, 407-421 (1990).

107. Ayala, A., Muñoz, M. F. \& Argüelles, S. Lipid Peroxidation: Production, Metabolism, and Signaling Mechanisms of Malondialdehyde and 4-Hydroxy-2-Nonenal. Oxid. Med. Cell. Longev. 1-31 (2014). doi:10.1007/978-3-211-33303-7_2

108. Yin, H., Xu, L. \& Porter, N. A. Free radical lipid peroxidation: Mechanisms and analysis. Chem. Rev. 111, 5944-5972 (2011).

109. Girotti, A. W. Lipid hydroperoxide generation, turnover, and effector action in biological systems. J. Lipid Res. 39, 1529-1542 (1998).

110. Evans, M. V., Turton, H. E., Grant, C. M. \& Dawes, I. W. Toxicity of linoleic acid hydroperoxide to Saccharomyces cerevisiae: Involvement of a respiration-related process for maximal sensitivity and adaptive response. J. Bacteriol. 180, 483-490 (1998).

111. Negre-Salvayre, A., Coatrieux, C., Ingueneau, C. \& Salvayre, R. Advanced lipid peroxidation end products in oxidative damage to proteins. Potential role in diseases and therapeutic prospects for the inhibitors. Br. J. Pharmacol. 153, 6-20 (2008).

112. Halliwell, B. \& Gutteridge, J. M. C. Oxygen toxicity, oxygen radicals, transition metals and disease. Biochem. J. 219, 1-14 (1984).

113. Renne, M. F. \& de Kroon, A. I. P. M. The role of phospholipid molecular species in determining the physical properties of yeast membranes. FEBS Lett. 592, 1330-1345 (2018).

114. Toomre, D., Simons, K. \& Toomre, D. Lipid rafts and signal transduction. Nat. Rev. Mol. Cell Biol. 1, 1 (2000).

115. Van Meer, G., Voelker, D. R. \& Feigenson, G. W. Membrane lipids: Where they are and how they behave. Nat. Rev. Mol. Cell Biol. 9, 112-124 (2008).

116. Tehlivets, O., Scheuringer, K. \& Kohlwein, S. D. Fatty acid synthesis and elongation in yeast. Biochim. Biophys. Acta - Mol. Cell Biol. Lipids 1771, 255-270 (2007).

117. Ackerman, D. et al. Triglycerides Promote Lipid Homeostasis during Hypoxic Stress by Balancing Fatty Acid Saturation. Cell Rep. 24, 2596-2605.e5 (2018).

118. Eisenberg, T. \& Büttner, S. Lipids and cell death in yeast. FEMS Yeast Res. 14, 179-197 (2014).

119. Czabany, T., Athenstaedt, K. \& Daum, G. Synthesis, storage and degradation of neutral 
lipids in yeast. Biochim. Biophys. Acta - Mol. Cell Biol. Lipids 1771, 299-309 (2007).

120. Wolf, A., Weir, P., Segar, P., Stone, J. \& Shield, J. Impaired fatty acid oxidation in propofol infusion syndrome. Lancet 357, 606-607 (2001).

121. Santer, R. et al. Tandem mass spectrometric determination of malonyl-carnitine: Diagnosis and neonatal screening of malonyl-CoA decarboxylase deficiency. Clin. Chem. 49, 660662 (2003).

122. Napolitano, N., Wiley, V. \& Pitt, J. J. Pseudo-glutarylcarnitinaemia in medium-chain acylCoA dehydrogenase deficiency detected by tandem mass spectrometry newborn screening. J. Inherit. Metab. Dis. 27, 465-471 (2004).

123. Rötig, A. \& Munnich, A. Genetic Features of Mitochondrial Respiratory Chain Disorders. J. Am. Soc. Nephrol. 14, 2995-3007 (2003).

124. Harris, J. R. \& Boekema, E. J. (Eds. . Membrane Protein Complexes: Structure and Function. Subcellular Biochemistry 87, (2018).

125. Cobine, P. A., Ojeda, L. D., Rigby, K. M. \& Winge, D. R. Yeast Contain A Nonproteinaceous Pool of Copper in the Mitochondrial Matrix. J. Biol. Chem. 279, 1444714455 (2004).

126. Mossine, V. V.\& Mawhinney, T. P. 1-Amino-1-deoxy-d-fructose ("Fructosamine”) and its Derivatives. Advances in Carbohydrate Chemistry and Biochemistry 64, (2010).

127. Mossine, V. V. \& Mawhinney, T. P. N-( 1-Deoxy-D-fructos-1-yl)-L-histidine ("D Fructose- L-histidine "): a Potent Copper Chelator from Tomato Powder. J. Agric. Food Chem. 55, 10373-10381 (2007).

128. Gyurcsik, B. et al. Proton, copper (II) and nickel (II) complexes of some amadori rearrangement products of d glucose and amino acids. Inorganica Chim. Acta 1, 57-66 (1993).

129. Luo, Y. et al. Effect of yeast cell morphology, cell wall physical structure and chemical composition on patulin adsorption. PLoS One 10, (2015).

130. Vijayarathna, S. et al. The antimicrobial efficacy of elaeis guineensis: Characterization, in Vitro and in Vivo Studies. Molecules 17, 4860-4877 (2012).

131. Jothy, S. L., Zakariah, Z., Chen, Y. \& Sasidharan, S. In vitro, in situ and in vivo studies on the anticandidal activity of Cassia fistula seed extract. Molecules 17, 6997-7009 (2012). 


\section{Appendix B}

\section{Supplemental Figures}
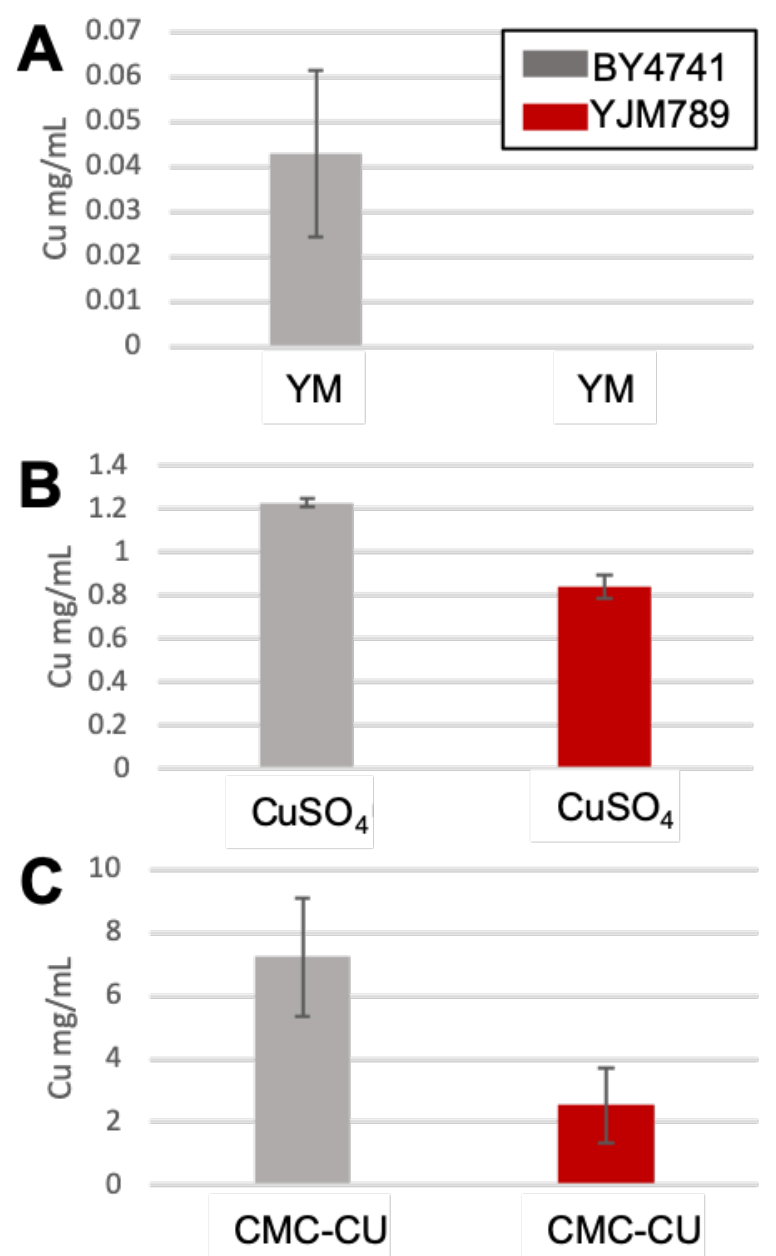

Figure 3-S1. Copper Homeostasis During CMC-Cu Toxicity Represented in Treatment Groups. (A) Copper levels after normal log phase cell growth in YM. (B) Copper levels after $\mathrm{CuSO}_{4}$ treatment. (C) Copper levels after $\mathrm{CMC}-\mathrm{Cu}$ treatment. Red bars represent the resistant strain BY4741. Silver bars represent the sensitive strain YJM789. 

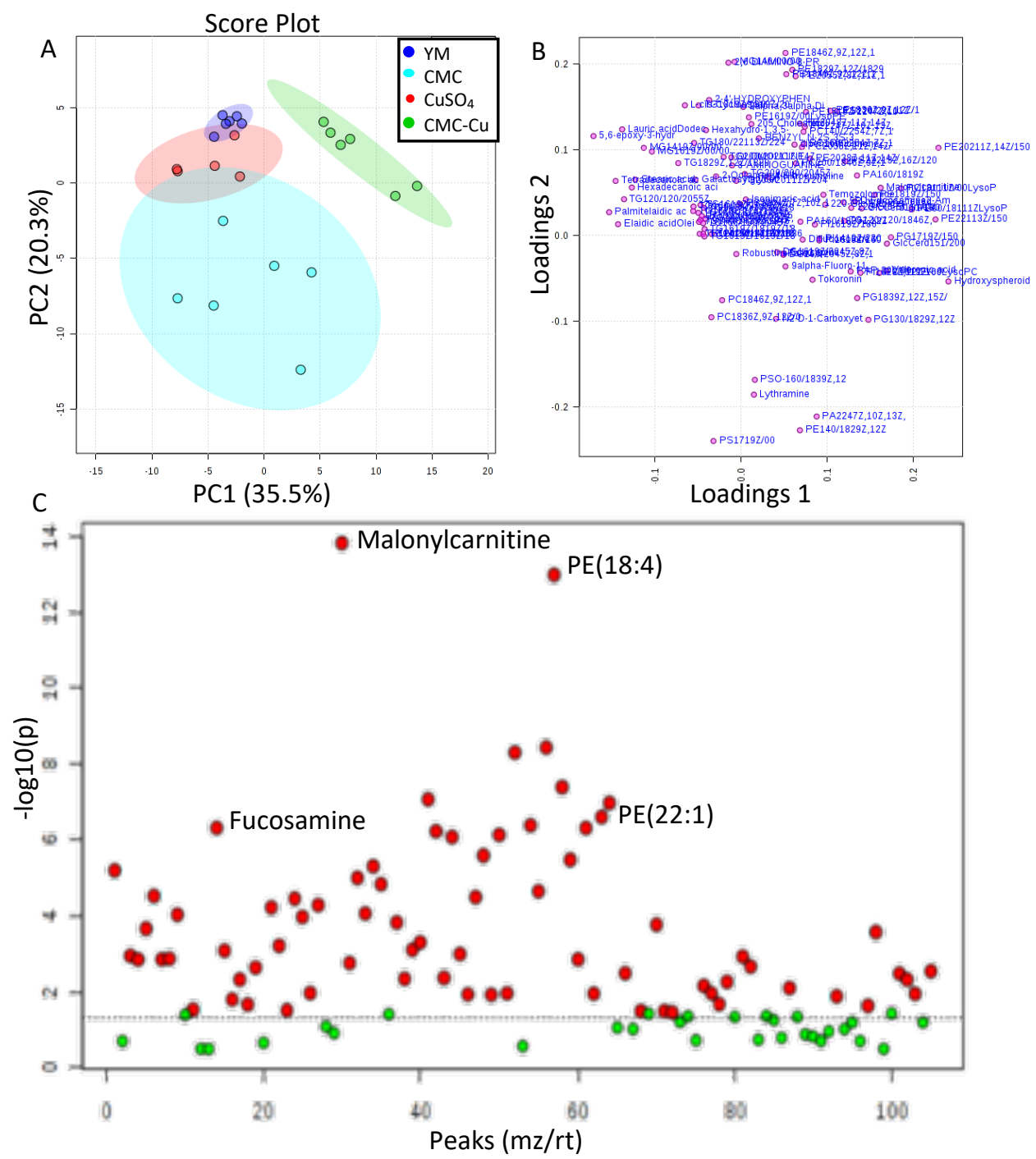

Figure 3-S2. Lipidomic Statistics for CMC-Cu Treatment. (A) Unsupervised principal component analysis (PCA) of yeast treatment with $\mathrm{CMC}-\mathrm{Cu}, \mathrm{CMC}, \mathrm{CuSO}_{4}$, and grown in YM media showing incomplete separation of all treatments. (B) Loading plot for the PCA seen in part B. (C) Important compounds identified by One-way ANOVA with a Tukey HSD post-hoc analysis. The significance is presented on the y-axis with red dots indicating a significance greater than $\mathrm{p}=0.05$ as indicated by the dashed line and insignificant dots in green. Of the compounds detected, select compounds of importance as identified in the heatmap analysis are labeled by name. 


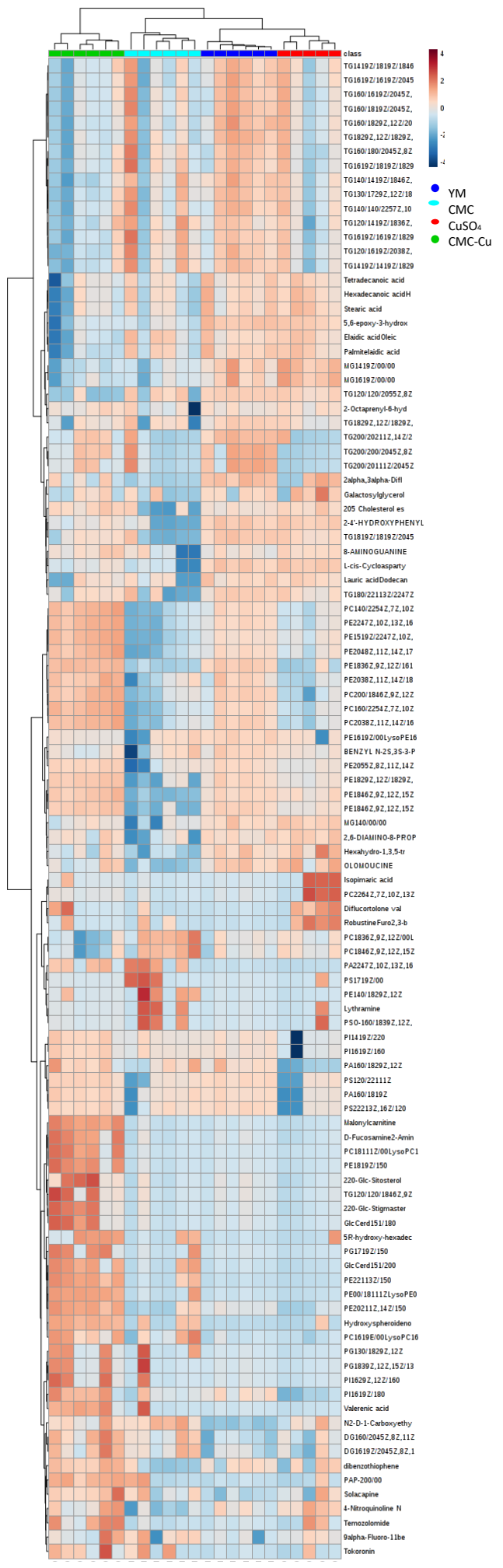

Figure 3-S3. Whole Lipidome Heatmap for CMC-Cu Treatment. Color indicates the relative abundance on a $\log 2$ fold change of the complete heatmap by each sample. 


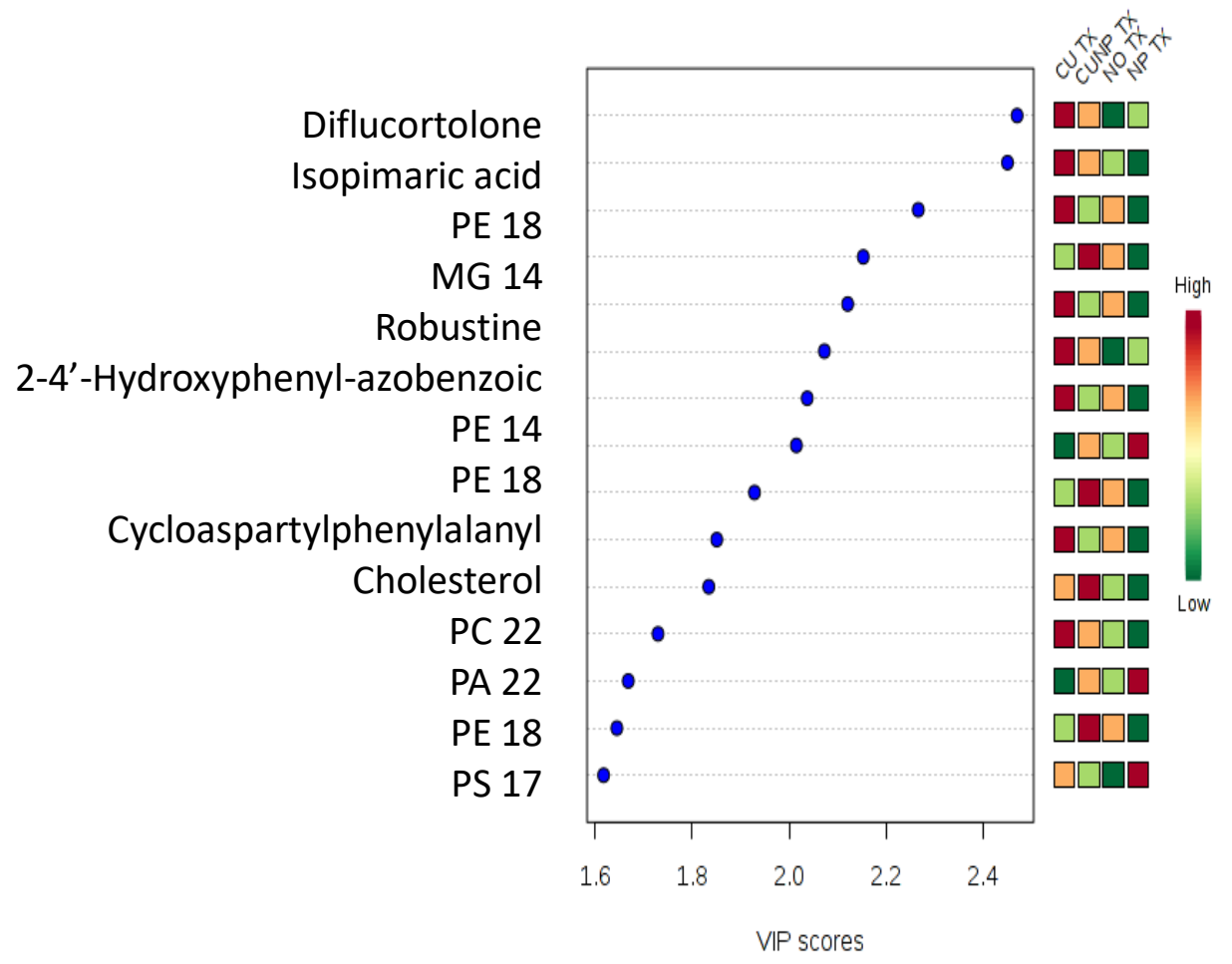

Figure 3-S4. Variable Importance Projection Score Plot. Color indicates the relative abundance on as a biomarker for each treatment. 


\section{Supplemental Tables}

Table 3-S1. Normalized Gas Chromatography Electrospray Ionization Mass Spectrometry Data.

Table 3-S2. Loading Matrix for PLS-DA Lipidomic Assay.

These supplemental tables can be accessed through this hyperlink. 


\title{
CHAPTER 4
}

\section{Saccharomyces arboricola and its Hybrids' Propensity for Sake Production: Interspecific Hybrids Reveal Increased Fermentation Abilities and a Mosaic Metabolic Profile}

An original research article published in Wine Fermentation 2.0, a special issue of Fermentation from the Multidisciplinary Digital Publishing Institute

Matthew J. Winans, Yuki Yamamoto, Yuki Fujimaru, Yuki Kusaba, Jennifer E.G. Gallagher, and Hiroshi Kitagaki

\begin{abstract}
The use of interspecific hybrids during the industrial fermentation process has been well established, positioning the frontier of advancement in brewing to capitalize on the potential of Saccharomyces hybridization. Interspecific yeast hybrids used in modern monoculture inoculations benefit from a wide range of volatile metabolites that broaden the organoleptic complexity. This is the first report of sake brewing by Saccharomyces arboricola and its hybrids. $S$. arboricola x $S$. cerevisiae direct-mating generated cryotolerant interspecific hybrids which increased yields of ethanol and ethyl hexanoate compared to parental strains, important flavor attributes of fine Japanese ginjo sake rice wine. Hierarchical clustering heatmapping with principal component analysis for metabolic profiling was used in finding low levels of endogenous amino/organic acids clustered $S$. arboricola apart from the $S$. cerevisiae industrial strains. In sake fermentations, hybrid strains showed a mosaic profile of parental strains, while metabolic analysis suggested $S$. arboricola had a lower amino acid net uptake than $S$. cerevisiae. Additionally, this research found an increase in ethanolic fermentation from pyruvate and increased sulfur metabolism. Together, these results suggest $S$. arboricola is poised for in-depth metabolomic exploration in sake fermentation.
\end{abstract}




\section{Introduction}

As currently defined, the budding yeast clade known as Saccharomyces has eight members: $S$. cerevisiae, S. paradoxus (syn. S. cariocanus, S. cerevisiae var. tetraspora, S. cerevisiae var. terrestris), S. uvarum (syn. S. bayanus var. uvarum), S. mikatae, S. kudriavzevii, S. arboricola (syn. S. arboricolus), S. eubayanus, and $S$. jurei ${ }^{1-9}$. Additionally, S. pastorianus (syn. $S$. carlsbergensis, $S$. monacensis) and $S$. bayanus are two natural hybrids found in this group ${ }^{10-12} . S$. arboricola was first isolated from the tree bark of the Fagaceea family in the west and southwest of China ${ }^{2}$ in 2008 and later also isolated in western Taiwan ${ }^{5}$ and northern New Zealand ${ }^{13}$. The New Zealand isolate was reported to be highly divergent from the Chinese isolates used in this study, supporting the far East Asian origin theory for Saccharomyces yeast. The Chinese $S$. arboricola has a high quality annotated genomic sequence that when compared to $S$. cerevisiae shows a reciprocal translocation on chromosome (chr.) IV and XIII, inversion on chr.VI, high presence of non-syntenic genes near telomeric regions, lack of introgression, and 4 novel genes ${ }^{14}$. Between $S$. cerevisiae and other Saccharomyces members there is a $15-30 \%$ divergence, but the divergence between $S$. cerevisiae strains is $0.1-0.6 \% 0^{15,16}$. Additionally, subpopulations of $S$. paradoxus show a $1.5-4.5 \%$ divergence, while the divergence between $S$. eubayanus and $S$. uvarum is $6 \%{ }^{7,17}$. The nucleotide divergence of yeast within Saccharomyces is near the man to bird comparison, but with the advantage of a physiological and structural similarity of man to chimp $^{18}$. Favorable properties of different Saccharomyces yeast can be brought together by hybridization that often results in transgressive phenotypes. Typical environmentally isolated Saccharomyces strains are diploid while laboratory strains are often used in the haploid form to reduce variability, and industrial yeast are frequently found to be polyploid, potentially a result of favored fermentation environment. Although interspecies hybrids of the Saccharomyces genus are viable, the they do not produce viable gametes or ascospores in yeast resulting in reproductive isolation as a sterility barrier ${ }^{9}$. Hybridization of phenotypically diverse yeast has been exploited for the improvement of industrial strains for specific purposes.

Fermenting yeasts, specifically $S$. cerevisiae, have been unwittingly domesticated throughout humanity's existence via the production of various foods and beverages, including bread, beer, wine, and sake. One factor in $S$. cerevisiae selection is its unique metabolic capabilities as a Crabtree positive yeast; they amass ethanol in the presence of oxygen. The commonly preferred eukaryotic metabolic pathway in the presence of oxygen is oxidative respiration for its high ATP yield. But in the high concentrations of six-carbon carbohydrates, such as glucose, this pathway is repressed by the Crabtree effect. Under these conditions, the energy for growth comes from the glycolysis pathway until exhaustion of six-carbon molecules causes a diauxic shift to oxidation of the two-carbon ethanol molecules into $\mathrm{CO}_{2}{ }^{19,20}$. Crabtree positive microbes are beneficial in the production of alcoholic beverages which have been produced for at least 9,000 years, and the earliest known recording of Japanese sake is mentioned ca. 285 AD in an ancient Chinese document called Gishiwajinden ${ }^{21-23}$. Sake is the alcoholic drink generated by fermenting rice and beer is that of barley, making their development specific to regions dominated by these grains. Rice and barley diverged 50 million years ago and rice was introduced to Japan from the Chinese Yangzi region around $400 \mathrm{BC}$, many differences exist between these two grains ${ }^{24,25}$. During the 15 th century in Bavaria, lager-brewing arose ${ }^{26}$ and is the most popular alcoholic beverage in the world today. Since the lager beer yeast, $S$. pastorianus was discovered to be a hybrid of $S$. eubayanus $\mathrm{x} S$. cerevisiae, interest has sparked in the use of interspecific hybrids in fermentation ${ }^{27}$. Originally the $S$. eubayanus parent was identified in Patagonia and the question of lager yeast's 
origin has generated controversy ${ }^{28-31}$. Yeasts originated multiple times from various fungal lineages of which one gave rise to the budding yeasts ${ }^{32}$. The genomic diversity with geographical boundaries and many other lines of evidence support a Far East Asia origin for Saccharomyces ${ }^{33-}$ 35 .

Beer, wine, and sake are complex chemical matrixes resulting from many metabolic and chemical reactions in which raw ingredients determine initial sugar, assimilable nitrogen, and fatty acid profiles $^{25,36}$. The volatile metabolic profile obtained during fermentation of different carbohydrates is reflective of the raw ingredients composition ${ }^{37,38}$. Wine is perhaps one of the oldest biotechnologies as yeast are often found on damaged grapes and crushing of the fruit facilitates the release of the fermentable sugars ${ }^{39,40}$. Obtaining fermentable sugars for beer and sake is quite different. Production of beer involves a saccharification of malted barley by the grain's own enzymes to convert their starches to maltose, glucose, and maltotriose found in descending concentrations. Boiling of the wort results in less than $3 \%$ of the malt lipids available to yeast during fermentation ${ }^{41}$. Fermentation of green beer is accomplished in 3 to 10 days ${ }^{42}$ and results in an average alcohol content of 5\% ( $\mathrm{vol} / \mathrm{vol})$ with some brews tipping the scales around 10\% (vol/vol) or more.

Immature sake is generally produced over 41 days and involves several steamed rice additions at temperatures between $12-20^{\circ} \mathrm{C}$ applying high osmotic pressure on the yeast from the rice mash $\operatorname{ratios}^{43}$. The highest alcohol yield in the world, $17-20 \%$ (vol/vol) without distillation, is obtained through this process ${ }^{25}$. Aspergillus oryzae, colloquially known as koji, is a keystone organism in the production of sake from polished rice ${ }^{44}$. By traditional Japanese sake mash methods, koji performs a continuous saccharification of highly milled rice starches to the fermentable sugars and the budding yeast, $S$. cerevisiae, ferments these sugars into ethanol ${ }^{45}$. Free sugars such as glucose and oligomers are produced by the koji enzymes glucoamylase and $\alpha$-amylase during the sake mash $^{23}$. Recent work has shown that koji secretes mannitol 2-dehydrogenase which converts fructose to mannitol in the sake mash. Interestingly, $S$. arboricola efficiently catabolizes mannitol, an abundant natural energy storage molecule that Aspergillus sp. produce in high concentrations ${ }^{46,47}$. This phenotype is superior to $S$. cerevisiae metabolism of mannitol ${ }^{14}$. Considering the temperature tolerance and growth capabilities on mannitol, it was hypothesized that $S$. arboricola and interspecific hybrids are uniquely fit for sake fermentation with $A$. oryzae because of their metabolic access to the underutilized sugar-alcohol and temperature preference which affects the flavor active metabolites.

The aim of this study was to characterize the metabolic capabilities of $S$. arboricola and its hybrids with $S$. cerevisiae in Japanese rice wine. For the first time, sake fermentation profiling of $S$. arboricola and newly generated $S$. arboricola $\mathrm{x} S$. cerevisiae interspecific hybrids is reported. The wine and beer brewing industries have often employed interspecific hybrids for their fermentations. $S$. kudriavzevii x $S$. cerevisiae are known to ferment wines, $S$. mikatae x $S$. cerevisiae hybrids have been actively produced for wine, and the $S$. cerevisiae x $S$. eubayanus hybrid produces the most popular beer in the world $33,48,49$. Limited research has been conducted on the metabolic capabilities of $S$. arboricola and these results suggest $S$. arboricola and the hybrids' propensity for fermentation may be suited for sake production. 


\section{Materials and Methods}

\section{Yeast Strains}

Yeast strains of Saccharomyces cerevisiae included Italian wine isolate M2250, West African wine isolate DBVPG6044 ${ }^{51}$, sake isolate Kyokai No. 7 known as haploid $868 \mathrm{~K} 7^{52}$, sake isolate TCR7 $7^{53}$, American brewing isolate WLP001, and American brewing isolate HT01. Yeast strains of Saccharomyces arboricola included SA350 (syn. AS 2.3317), SA351 (syn. AS 2.3318), SA352 (syn. AS 2.3319 ${ }^{14}$. Hybrids SAM8c and SAM9a were between S. arboricola 350 X S. cerevisiae M22 (Table 4-1). A minimum of three biological replicates were used in each analysis.

\section{Yeast Isolation}

Table 4-1. Yeast Strains Used During Hybridzation Study

\begin{tabular}{|c|c|c|c|}
\hline Strain & Species & Isolation source & Reference \\
\hline $\begin{array}{c}\text { SA350 (syn. AS } \\
2.3317 \text { ) }\end{array}$ & S. arboricola & Tree bark - China & Liti, G., et al. (2013) \\
\hline $\begin{array}{c}\text { SA351 (syn. AS } \\
2.3318 \text { ) }\end{array}$ & S. arboricola & Tree bark - China & Liti, G., et al. (2013) \\
\hline $\begin{array}{c}\text { SA352 (syn. AS } \\
2.3319 \text { ) } \\
\end{array}$ & S. arboricola & Tree bark - China & Liti, G., et al. (2013) \\
\hline M22 & S. cerevisiae & Wine - Italy & $\begin{array}{c}\text { Capece, A., et al. } \\
\text { (2012) }\end{array}$ \\
\hline DBVPG6044 & S. cerevisiae & Billi wine - West Africa & Song, G., et al. (2015) \\
\hline $\begin{array}{c}\text { Kyokai No. } 7 \text { (syn. } \\
\text { 868K7/ K7) }\end{array}$ & S. cerevisiae & Sake - Japan & Katou, T., et. al. (2008) \\
\hline TCR7 & S. cerevisiae & Sake - Japan & Horie, K., et al. (2010) \\
\hline WLP001 & S. cerevisiae & Beer, American ale & $\begin{array}{c}\text { Commercially } \\
\text { available from White } \\
\text { Labs, USA } \\
\end{array}$ \\
\hline HT01 & S. cerevisiae & Beer, American ale & Current study \\
\hline SAM8c & $\begin{array}{c}\text { Hybrid }(S . \\
\text { arboricola } x S . \\
\text { cerevisiae) }\end{array}$ & $\begin{array}{l}\text { Lab, spore to spore } \\
\text { mating }\end{array}$ & Current study \\
\hline SAM9a & $\begin{array}{c}\text { Hybrid }(S . \\
\text { arboricola } x S . \\
\text { cerevisiae) }\end{array}$ & $\begin{array}{c}\text { Lab, spore to spore } \\
\text { mating }\end{array}$ & Current study \\
\hline
\end{tabular}


Saccharomyces cerevisiae HT01 was the only strain isolated for this study and was isolated as described previously ${ }^{54}$ with slight modification. Briefly, beer was harvested from a can sanitized on the outside with $70 \%$ ethanol into an enrichment medium ( $3 \mathrm{~g}$ yeast extract, $3 \mathrm{~g}$ malt extract, 5 g peptone, $10 \mathrm{~g}$ sucrose, $76 \mathrm{ml} \mathrm{EtOH}, 1 \mathrm{mg}$ chloramphenicol, and $1 \mathrm{ml}$ of 1-M HCl per liter). Samples were transferred to sterile tubes and incubated until signs of yeast appeared. Cultures were then streaked onto YPD media and single colonies were grown and stocked. From these stocks, the yeast culture's genomic DNA was extracted, the ITS region was amplified via polymerase chain reaction $(\mathrm{PCR})^{55}$, Sanger sequenced at the West Virginia University Genomic Core, and the isolated yeast was confirmed to be S. cerevisiae via utilizing NCBI Blast.

\section{Serial Spot Dilutions}

Serial spot dilution growth assays were conducted as described previously ${ }^{56}$ with some modification. Briefly, $\mathrm{OD}_{600 \mathrm{~nm}}$ growth readings were taken from fresh, overnight cultures of each yeast grown at $23^{\circ} \mathrm{C}$. For each yeast assayed, a total of $3.2 \times 10^{5}$ yeast cells were transferred to a well in the first column of a 96 well plate. Each yeast assayed was serially diluted 4 times in a 1:10 ratio, using sterile distilled and MilliQ (MQ) filtered water, across the rows which resulted in 5 wells each. A handheld 48 pin plate was used to place the serial dilution spots onto solid YPD agar media. Plates were then incubated at $4^{\circ} \mathrm{C}$ for ten days, $10^{\circ} \mathrm{C}$ for seven days, and $15^{\circ} \mathrm{C}$ for four days. Growth was assessed and photographed.

\section{Sporulation and Spore Staining}

Fresh liquid yeast cultures were grown in YPD (1\% yeast extract, $2 \%$ peptone, $2 \%$ dextrose) overnight at $23^{\circ} \mathrm{C}$ in a shaking water bath. Isolates were streaked out onto YEP(EG) buffered agar plates $(1 \%$ succinic acid, $1 \%$ yeast extract, $2 \%$ peptone, $2 \%$ glycerol, $2 \%$ agar, potassium hydroxide to $\mathrm{pH} 5.5,2.5 \%$ ethanol) for 2-3 days. Single colonies were thinly patched onto SnackSpo $\mathrm{Zn}$ agar plates ( $1 \%$ potassium acetate, $0.1 \%$ yeast extract, $0.05 \%$ dextrose, $0.005 \%$ zinc acetate, $2 \%$ agar) and incubated for 6 days. To measure spore formation, a modified SchaefferFulton endospore staining technique was used. Briefly, cultures from the sporulation plates were suspended in $1 \mathrm{ml}$ distilled water and recovered by pipette. The suspended culture was fixed to a glass microscope slide via flame. Malachite green $(10 \%, 0.1 \mathrm{~g} / \mathrm{ml})$ was then used to stain the spore walls for $15 \mathrm{~min}$ (minute/s) before decoloring and rinsing with water. Safranin $(0.25 \%, 2.5 \mathrm{mg} / \mathrm{ml})$ was used as a counterstain for non-sporulated cells for $1 \mathrm{~min}$ before rinsing with water and mounting in glycerol. Malachite green cells were counted as spores and safarin red cells were counted as non-sporulated yeast cells ${ }^{57}$. Images were taken under a light microscope and sporulation efficiency for each strain was calculated, reported in the results section, to select the best possibility of a successful hybridization.

\section{Interspecific Hybridization}

The ascus of the spore was digested over $1 \mathrm{hr}$. with Zymolase $(25 \mathrm{mg} / \mathrm{ml})$ at $37^{\circ} \mathrm{C}$. The released spores were placed dropwise on YPD agar at an angle to facilitate a central streak of liquid across the plate. Micromanipulation of spores from S. arboricola strain 350 and S. cerevisiae M22 spores was performed by fiber optic needle under a light microscope using the SporePlay (Singer Instruments, Roadwater, England) dissection microscope by Singer Instruments and yielded 40 spore to spore mattings. Visual observations were conducted on the shmooing process during 
incubation at $25^{\circ} \mathrm{C}$. Single colonies were selected, streaked out for purification, and stocked at $80^{\circ} \mathrm{C}$ with cryogenic media ( $1 \%$ yeast extract, $2 \%$ peptone, $15 \%$ glycerol).

\section{Genomic DNA Extraction}

Alkaline lysis genomic DNA extraction was performed on fresh, overnight cultures by harvesting $\left(0.3 \times 10^{7}\right)$ cells via cold centrifugation at $7,500 \mathrm{xg}$ for $3 \mathrm{~min}$. Cells were washed twice with phosphate-buffered solution (PBS) before $\mathrm{NaOH}(0.02 \mathrm{M})$ addition and subsequent incubation at $100^{\circ} \mathrm{C}$ for $10 \mathrm{~min}$. The cellular solution underwent cold centrifugation at $4^{\circ} \mathrm{C}$ for 3 min before $250 \mu \mathrm{l}$ of supernatant was mixed with $50 \mu \mathrm{l}$ acetic acid $(2.5 \mathrm{M})$ and then $300 \mu \mathrm{l}$ of 2-propanol. Cold centrifugation was performed again for $15 \mathrm{~min}$ before the supernatant was discarded. Ethanol (80\% $\mathrm{v} / \mathrm{v}$ ) was added to the DNA pellet to lightly mix the solution before a 5 min cold centrifugation. The DNA was dried at room temperature and resuspended in TE buffer.

\section{Polymerase Chain Reaction}

DNA concentrations were measured by $\mathrm{OD}_{260 \mathrm{~nm}}$ via a glass black cell cuvette. PCR confirmed $\underline{S}$. arboricola 350 and $S$. cerevisiae $\underline{\mathrm{M}} 22$ (SAM hybrids) interspecific hybridization. Two multiplexed species-specific primer sets were used, detailed previously ${ }^{58}$. The oligonucleotide sequences used for primers were as follows; for $S$. arboricola sarbF1(GGC ACG CCC TTA CAG CAG CAA TCG) and sarbR2 (TCG TCG TAC AGA TGC TGG TAG GGC GCT); for $S$. cerevisiae scerF2 (GCG CTT TAC ATT CAG ATC CCG AG) and scerR2 (TAA GTT GGT TGT CAG CAA GAT TG ATC). A master mix for the PCR reactions was made on ice and consisted of PCR reagents from kit: KOD-Plus-Neo (product code KOD-401) ordered from TOYOBO, Japan. The PCR Touchdown ${ }^{59}$ thermocycle protocol was used. During gel electrophoresis ${ }^{60}$, the S. cerevisiae band generated at $150 \mathrm{bp}$ (base pairs) and $S$. arboricola generated at $349 \mathrm{bp}$.

\section{Sake Fermentations}

Freshly grown cells $\left(2.0 \times 10^{9}\right)$ were measured by $\mathrm{OD}_{600 \mathrm{~nm}}$, harvested from YPD via centrifugation at 3,200xg for $3 \mathrm{~min}$, and washed in MilliQ water twice. The harvested yeast, rice, koji, lactate, and water were mixed together in mason jars covered with loose lids. Incubation occurred at $15^{\circ} \mathrm{C}$ for 2 weeks with an initial remixing of the mash occurring after the first 24 hours. Fermentation was monitored daily by mass reduction of the sake mash, which corresponded to $\mathrm{CO}_{2}$ production. Fermentation trials were performed in triplicate. Post-fermentation, the sake mash was harvested into conical tubes by centrifugation at $4^{\circ} \mathrm{C}$ and $3,200 \mathrm{x}$ g. The supernatant was transferred to storage at $4^{\circ} \mathrm{C}$ for further analysis. Statistical analysis of ethanol content was validated by oneway ANOVA with a two-tailed student's t-test post-hoc analysis and ordered difference report for each day of fermentation.

\section{Chromatography Analysis}

Gas Chromatography-Flame Ionization Detection

For gas chromatography-flame ionization detection (GC-FID) of the sake supernatant, ribitol (syn. adonitol) $(0.2 \mathrm{mg} / \mathrm{ml})$ was supplied as an internal standard. Samples were freeze-dried via liquid nitrogen bath followed by vacuum chamber drying for $6 \mathrm{hrs}$. The freeze-dried samples were mixed with $100 \mu \mathrm{l}$ of methoxylamine diluted with pyridine $\left(20 \mathrm{mg} / \mathrm{ml}\right.$ ] and incubated at $30^{\circ} \mathrm{C}$ for $90 \mathrm{~min}$ with agitation (1,500rpm). The sample was mixed with $50 \mu \mathrm{l}$ of N-methyl-N-TMStrifluoroacetamide (MSTFA) incubated for $30 \mathrm{~min}$ at $37^{\circ} \mathrm{C}$ with agitation and transferred into a 
glass vial for chromatography metabolome analysis. A Cp-sil8CB column from Agilent, model \#CP8751 (30mm, $0.25 \mathrm{~mm}, 0.25 \mu \mathrm{m})$ was used to assess the sake fermentation samples. The injection was at $230^{\circ} \mathrm{C}$ with a $1 \mathrm{ml} / \mathrm{min}$ helium carrier gas flow rate. The column temperature was held at $80^{\circ} \mathrm{C}$ for $2 \mathrm{~min}$ before rising $10^{\circ} \mathrm{C} / \mathrm{min}$ until $320^{\circ} \mathrm{C}$ was achieved, the temperature was held for $12 \mathrm{~min}$ before reaching a $320^{\circ} \mathrm{C}$ detector. The Shimadzu Corporation (Shimadzu Manufacturing company, Kyoto, Japan) model GC-2014AFSC 100V was used in performing the GC-FID analysis.

\section{Head Space-Solid Phase Microextraction}

For the head space-solid phase microextraction (HS-SPME), the sake supernatant samples were prepared with $\mathrm{NaCl}$ and methyl hexanoate $(40 \mathrm{mg} / 100 \mathrm{ml})$ was supplied as an internal standard. In a glass vial, the solution was agitated under $80^{\circ} \mathrm{C}$ heat with a stir bar (160rpm) for $15 \mathrm{~min}$. A syringe

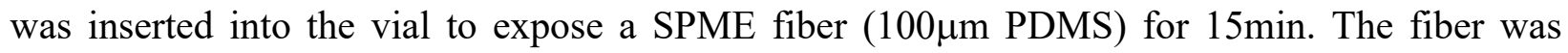
retracted into the syringe before removal from the vial and inserted into the injector for a $5 \mathrm{~min}$ exposure. A DB wax column from Agilent, model \#122-7032 (30 mm, 0.25mm, $0.25 \mu \mathrm{m})$ was used. The injection was at $200^{\circ} \mathrm{C}$ with a $0.95 \mathrm{ml} / \mathrm{min}$ helium carrier gas flow rate. The column temperature was held at $60^{\circ} \mathrm{C}$ for $1 \mathrm{~min}$ before rising $3^{\circ} \mathrm{C} / \mathrm{min}$ until $100^{\circ} \mathrm{C}$ was achieved, the rate increased to $15^{\circ} \mathrm{C} / \mathrm{min}$ until $200^{\circ} \mathrm{C}$ was reached and held for $4 \mathrm{~min}$ before reaching a $320^{\circ} \mathrm{C}$ detector. The Shimadzu Corporation (Shimadzu Manufacturing company, Kyoto, Japan) model GC-2014AFSC 100V was used in performing the HS-SPME analysis.

\section{Gas Chromatography-Mass Spectroscopy}

For gas chromatography-mass spectroscopy (GC-MS) of endogenous metabolites, stationary phase yeast cultures were grown in YPD liquid media. Stationary phase was defined at an $\mathrm{OD}_{600 \mathrm{~nm}}$ of 2.0. Cells $\left(4 \times 10^{7}\right)$ were harvested, washed, and freeze-dried. An extraction solution (chloroform:methanol:MQ [2:5:2]) with ribitol $(0.2 \mathrm{mg} / \mathrm{ml})$ as the internal standard was added to the freeze-dried samples and incubated for 30 minutes at $37^{\circ} \mathrm{C}$ under agitation $(1500 \mathrm{rpm})$. The samples were centrifuged, and the supernatant was harvested. MQ water was added to the supernatant and centrifuged. The supernatant was concentrated under vacuum centrifugation ($100 \mathrm{kPa}$ ) at $37^{\circ} \mathrm{C}$. MQ water was added, and the water-soluble metabolites were freeze-dried. Methoxyamine $(20 \mathrm{mg} / \mathrm{ml})$ was added to the samples and incubated for $90 \mathrm{~min}$ at $30^{\circ} \mathrm{C}$ under agitation (1500rpm). MSTFA (N-methyl-N-trimethylsilyl trifluoroacetoamide) was added to the samples and incubated for $30 \mathrm{~min}$ at $37^{\circ} \mathrm{C}$ with agitation (1500rpm). Samples were supplied in glass vials for GC-MS analysis using an Agilent DB-5MS+DG (30m, 0.25mm, 0.25um) column, splitless injection, and helium carrier gas $(1 \mathrm{ml} / \mathrm{min})$. The column temperature rose from $80^{\circ} \mathrm{C}$ at a rate of $13^{\circ} \mathrm{C} / \mathrm{min}$ until reaching a $320^{\circ} \mathrm{C}$ detector. The Agilent Technologies, Inc. (Agilent Technologies, Santa Clara, California, United States of America) model 7890B was used for the GC system, model 5977A was used for the MS system, and the National Institute for Standards and Technology (NIST) mass spectral library was used.

\section{Data Analysis}

The peak area of identified compounds was divided by the peak area of the internal standard for normalization of the data. Normalized peak areas of the compounds and ethanol concentrations measured during sake fermentations were auto-scaled and used as independent variables in the loading plots for construction of the Principal Component Analysis (PCA). Statistical Analysis was performed by soft independent modeling by class analogy (SIMCA) methodology by SIMCA 
Version 13.0.3.0 (Umetrics, Umeå, Sweden) computer software. Analysis of variance (ANOVA), post-hoc student's t-test, and post hoc Tukey-Kramer honest significance test (Tukey HSD), $\mathrm{p}<0.05$ was used for statistical significance. Deviations from this statistical analysis procedure, if occurred, was reported in individual results or methods sections. Heatmap was generated through MetaboAnalyst $4.0^{61}$. Heatmaps were based on data normalization by internal reference feature, cubed root transformation, pareto/range data scaling for endogenous/sake metabolites, clustering by Ward's method, and Euclidean distance measurements.

\section{Results}

Endogenous metabolome characterization of $S$. arboricola was put in context of industrially relevant $S$. cerevisiae yeast. Two yeast strains were chosen to represent either beer, wine, or sake fermentation industries. WLP001 and HT01 represented beer, M22 and DBVPG6044 represented wine, K7 and TCR7 represented sake production. SA350, SA351, and SA352 were used to represent wild S. arboricola (Table 3-1). Endogenous metabolite profiling was visualized in a hierarchical clustering heatmap that showed clustering of yeast by industry and species (Figure 41A). Ward's linkage clustering algorithm created two main clusters separated by species with the exception of West African bili wine strain DBVPG6044 that grouped within S. arboricola. The yeast in this cluster, located in the right half of the heatmap, all showed a low concentration of endogenous amino acids. Beer strains, sake strains, and wine strain M22 composed the other cluster on the left half. Beer strains paired together, and sake strains paired together with Italian wine strain M22. These industrial strains had similar but varying metabolic profiles displaying high endogenous levels of many amino and organic acids.

A score plot generated through principal component analysis separated the strains into general industry-specific groups (Figure 4-1B). A total of 19 metabolites identified by GC-MS were used as variables in the construction of the loading plot (Figure 4-1C). Sugar utilization played a major role in separation of the strains. $S$. arboricola strains were grouped to the left while beer and sake strains grouped opposite to the right on principal component (PC)1 explaining $36.2 \%$ of the sample variance and separating the groups by species. On the axis of PC2, sake yeast grouped high, wine yeasts were neutral/variable, and beer yeasts were low explaining $18.0 \%$ of sample variance and separating the yeast by their industrial niche domestication. Glucose, sucrose, and trehalose negatively contributed to $\mathrm{PC} 1$, which exemplified sugar uptake differences and was inferred to translate to fermentation aptitude. Beer brewing strains were found to be the most inclined yeasts in this regard and S. arboricola were found to be the least adept. A third component, explaining $12.7 \%$ was used to create a 3D PCA plot of the endogenous metabolites (Figure 4-S1). Citric acid, malic acid, fumaric acid, glutamic acid, nicotinic acid, succinic acid, aspartic acid, threonine, serine, glycine, phenylalanine, pyroglutamine, methionine, uracil, inositol, and adenine positively contributed to PC1. A loading matrix was constructed for the variables (Table 3-S1). Glycine, threonine, malic acid, fumaric acid, nicotinic acid, succinic acid, sucrose, trehalose, inositol, and adenine positively contributed to PC2. Phenylalanine, pyroglutamine, methionine, uracil, aspartic acid, and glucose contributed negatively to PC2, which distinguished the beer brewing strains from the sake strains. These two groups are represented in the heatmap's industrial cluster. Factors from the heatmap show the difference between sake and beer yeast include the endogenous inositol 

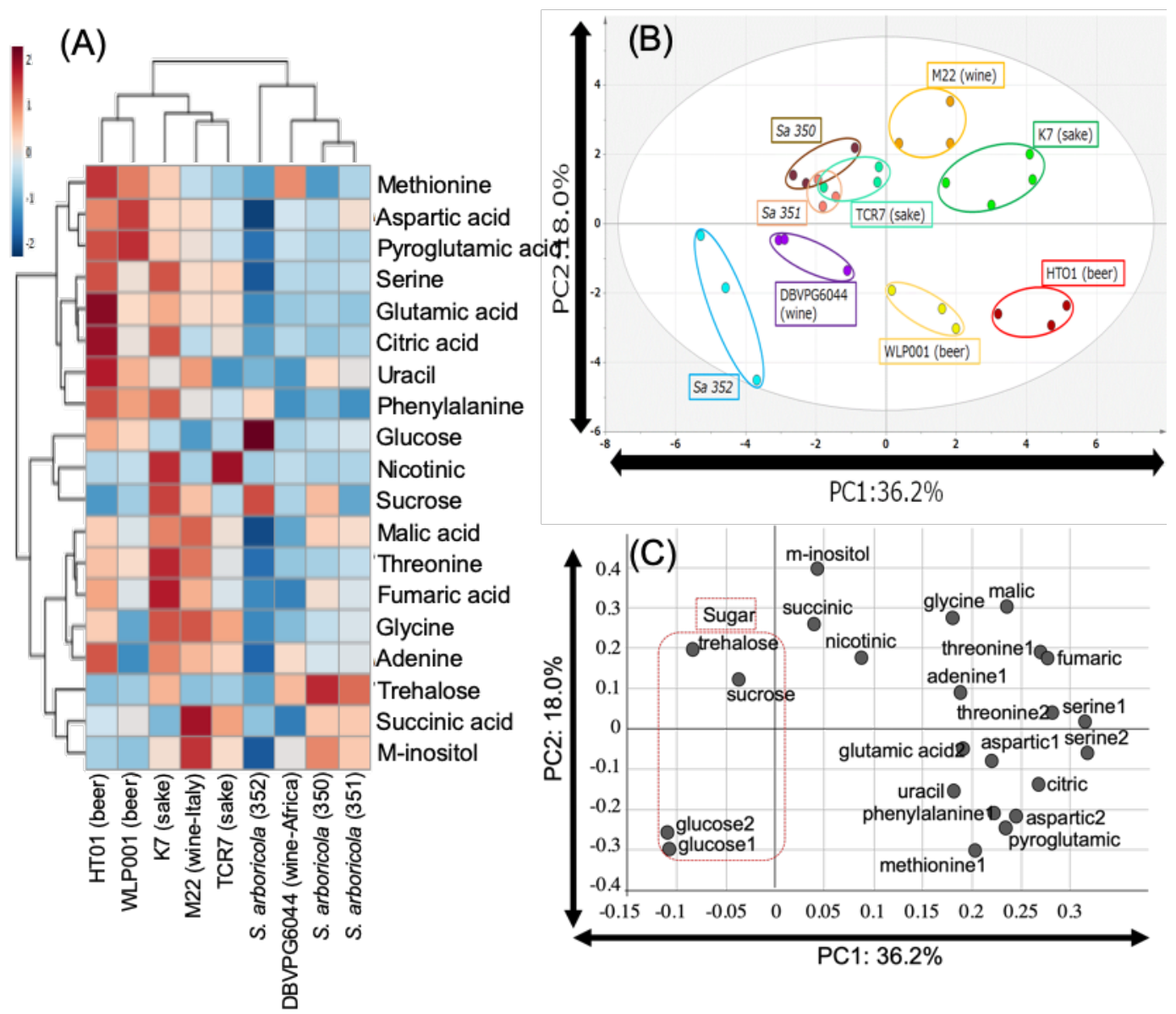

Figure 4-1. Yeasts Endogenous Metabolic Profiles. (A) Hierarchical clustering heatmap of endogenous metabolite profiles between strains and species. Relatively high compound concentrations were shown in varying degrees of red. Relatively low compound concentrations were shown in varying degrees of blue. Shown as average concentration per sample groupings of three replicates. (B) PCA score plot of Saccharomyces yeast explaining $17.4 \%$ of the variance on PC2 and $31.6 \%$ of the variance on PC1. (C) PCA loading plot. Saccharide content is circled to show importance in the PC1 variance. PCA and loading plot generated through SIMCA analysis. Three replicates were used for each strain.

levels; which were high in sake yeast and affect membrane fluidity during osmotic stress such as an industrial sake mash.

The potential of Saccharomyces hybridization to broaden metabolic capabilities and sensory complexity from volatile metabolites was explored using

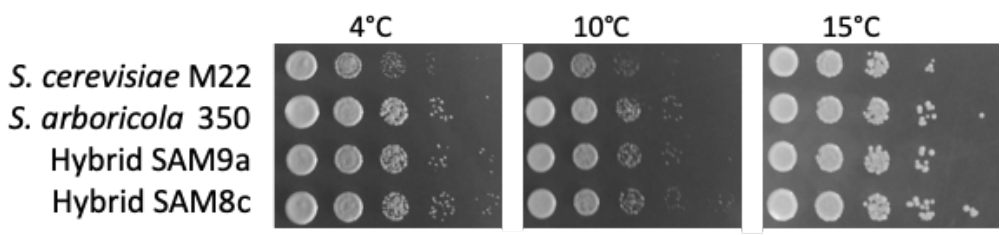
Figure 4-2. Cryotolerance of Parental and Hybrid Yeasts. Serial spot dilution growth assay of parental S. cerevisiae M22, parental S. arboricola 350, and the SAM hybrids. Three different temperatures of $4^{\circ} \mathrm{C}, 10^{\circ} \mathrm{C}$, and $15^{\circ} \mathrm{C}$ was used in assessment of their cryotolerance. Yeast were incubated on solid YPD agar for ten days at $4^{\circ} \mathrm{C}$, seven days at $10^{\circ} \mathrm{C}$, and four days at $15^{\circ} \mathrm{C}$. S. arboricola and S. cerevisiae. 
Sporulation efficiency was used in selection of strains for direct-mating hybridization as industrial strains are typically poor sporulators. $S$. arboricola 350 and S. cerevisiae M22 yielded the highest sporulation efficiency at $69 \%$ and $91 \%$ respectively (Figure 4-S2A). Selection of the $S$. cerevisiae industrial strain relied on sporulation results to optimize spore-spore mattings and given M22 grouped similar to the sake strain metabolism in Figure 4-1B, M22 was chosen. Other S. arboricola strains sporulated at $68 \%$ for 351 and $20 \%$ for 352 . Interspecific SAM ( $\underline{S}$. arboricola 350 and $S$. cerevisiae $\underline{\mathrm{M}} 22$ ) hybrids, SAM8c \& SAM9a, generated double bands representing genomic input from both species. Two spore-to-spore mating colonies were advanced to lab-scale sake fermentation after confirmation of hybrid state by species-specific multiplexed PCR (Figure 4-S2B). Cryotolerance of $S$. arboricola and the hybrids was shown as substantial growth at $4^{\circ} \mathrm{C}$ and $10^{\circ} \mathrm{C}$ after ten and seven days, respectively (Figure 4-2). At $15^{\circ} \mathrm{C}$, there was no perceivable difference between yeast growth. Thermosensitivity was not detected using the serial spotting dilution assay in temperatures up to $30^{\circ} \mathrm{C}$, slow growth of $S$. arboricola cultures during extreme temperature highs in the summer months was observed.

To determine if important sake flavor molecules changed with hybridization, ethyl hexanoate, isoamyl acetate, and ethanol fermentation was measured. In fact, it was found that S. arboricola, $S$. cerevisiae, and the hybrids metabolite profiles after lab-scale sake mash fermentation trials were different. The hybrids had a more vigorous fermentation, yielding a higher alcohol content than $S$. cerevisiae by day seven $(p=0.031)$ of the 14-day trial fermentation (Figure 4-3A). A oneway ANOVA with a two-tailed student's t-test post-hoc analysis and ordered difference report for each day of fermentation was generated to determine ethanol content significance (Table 3S2). The gap between fermentation rates declined over time trending towards non-significance between all samples if more time was allotted.
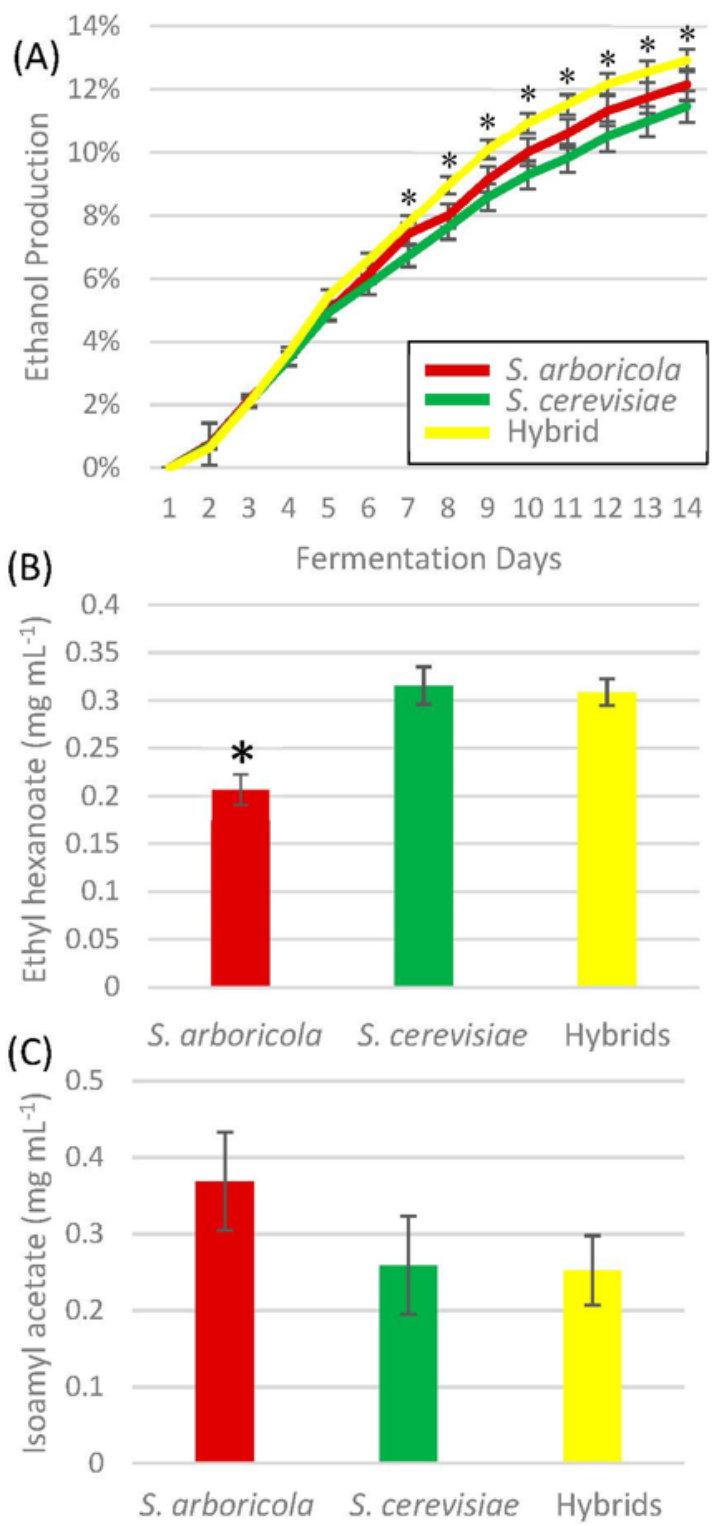

Figure 4-3. Phenotypic Characteristics of Hybrids and Parental Yeast. (A) Mean alcoholic fermentation rates of S. arboricola 350, S. cerevisiae M22, and the SAM hybrids are shown as a line graph representing the alcohol content over 14 days in time. Standard error bars shown in line graph (B) Bar chart depicting the production of ethyl hexanoate in $\mathrm{mg} \mathrm{mL}^{-1}$. (C) Bar chart depicting the production of isoamyl acetate in $\mathrm{mg} \mathrm{mL}^{-1}$. Statistical analysis performed by ANOVA, post-hoc student's T-test, and post-hoc TukeyHSD, $\mathrm{p}<0.05$. 
The fermentations were stopped as the fermentation rate slowed down and a fair amount of ethanol $10 \%+$ was produced. The fermentation trials were designed with time constraints as this is often a major factor in production settings and attenuation was secondary. Differences in the final alcohol yield at day 14 was significant between $S$. cerevisiae and the hybrids $(\mathrm{p}=0.038)$, while the value for $S$. arboricola was not significantly different than either hybrid nor $S$. cerevisiae. Hybrid SAM9a had the most productive fermentation rate, followed by hybrid SAM8c, S. arboricola 350, and $S$. cerevisiae M22. The mean final alcohol content of sake produced was $12.92 \%$ for the hybrids, $12.15 \%$ for $S$. arboricola 350 , and $11.46 \%$ for S. cerevisiae M22.

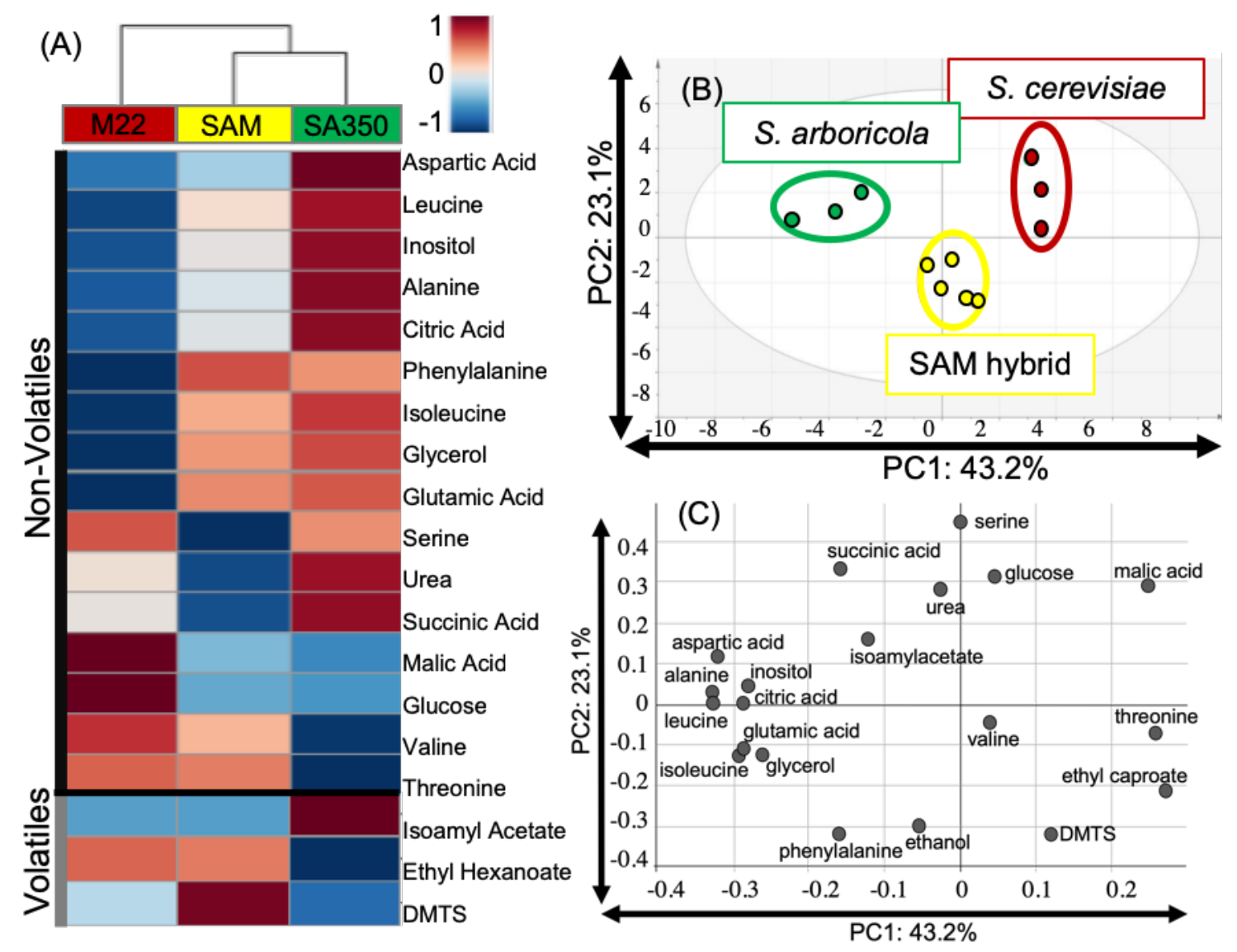

Figure 4-4. Sake Fermentation Profiles of S. arboricola Interspecific Hybrids and Parental Strains. (A) Hierarchical clustering heatmap of sake fermentation profiles between strains and species. Relatively high compound concentrations were shown in varying degrees of red. Relatively low compound concentrations were shown in varying degrees of blue. Shown as average concentration per sample groupings of three replicates each. (B) PCA score plot of sake fermentation profiles obtained for S. cerevisiae, S. arboricola, and the SAM hybrids. (C) PCA loading plot of sake fermentation profiles obtained for S. cerevisiae, S. arboricola, and the SAM hybrids. PC1 explained $43.2 \%$ and PC2 explained $23.1 \%$ of sample variance. PCA and loading plot generated through SIMCA analysis. Three replicates were used for each strain.

Detection of volatile aromatic compounds allows insight into flavor and sensory attributes of the final sake produced and HS-SPME technology in the gas chromatography system was utilized. Sake fermented with $S$. arboricola contained significantly less ethyl hexanoate (syn. ethyl 
caproate) $\left(0.207 \mathrm{mg} \mathrm{mL}^{-1}\right.$, st error 0.016$)$ than $S$. cerevisiae $\left(0.316 \mathrm{mg} \mathrm{mL}^{-1}\right.$, st error $0.019, \mathrm{p}=$ $0.005)$, or the hybrids $\left(0.308 \mathrm{mg} \mathrm{mL}^{-1}\right.$, st error $\left.0.014, \mathrm{p}=0.003\right)$ (Figure 4B). Sake fermented by $S$. arboricola contained a concentration of isoamyl acetate $\left(0.368 \mathrm{mg} \mathrm{mL}^{-1}\right.$, st error 0.064$)$ that was high compared to $S$. cerevisiae $\left(0.259 \mathrm{mg} \mathrm{mL}^{-1}\right.$, st error $\left.0.064, \mathrm{p}=0.25\right)$ and the hybrids $(0.252$ $\mathrm{mg} \mathrm{mL}^{-1}$, st error $0.045, \mathrm{p}=0.17$ ), but fell short of a significant $\mathrm{p}$-value (Figure 4-3C). Ethyl hexanoate imparts apple or aniseed and isoamyl acetate imparts a banana-like attribute to human sensory receptors. Whole metabolite heatmap by sample was created showing a separation of $S$. arboricola from the hybrids and M22 individual samples when volatile compounds were used (Figure 4-S3A) or a separation of all three when non-volatile metabolites were used (Figure 4S3B).

Sake fermentation profiles were visualized in a hierarchical clustering heatmap with hybrids clustering in closer proximity to the $S$. arboricola yeast (Figure 4-4A). Parental strain $S$. cerevisiae M22's sake metabolite profile contained high amounts of glucose, malic acid, threonine, and valine while parental $S$. arboricola's profile contained high amounts of citric acid, aspartic acid, glutamic acid, leucine, isoleucine, alanine, phenylalanine, inositol, and glycerol. Parental profiles almost completely opposed one another with only serine, succinic acid, and urea showing similar parental concentrations. M22 was the most distant parent to the hybrids' profile. The heatmap of the hybrid strains showed a mosaic blending of the two parental species. Metabolomic analysis using principal components clearly separated interspecific hybrids from each parent based in a PCA score plot (Figure 4-4B). A total of 20 metabolites, including 17 non-volatile compounds, were identified by GC-FID and 3 volatile compounds identified by HS-SPME These were used as variables in construction of the loading plot (Figure 4-4C). Principal component (PC1) separated samples by their species and explained $43.2 \%$ of the variance between the samples. Fermentation profiles showed a separation of the two Saccharomyces species on opposite ends of PC1 and the hybrid yeast grouped to the middle. PC2 separated the hybrid from the parental samples by the novel hybrid fermentation features that explained $23.1 \%$ of the sample variance. Threonine, valine, malic acid, glucose, ethyl hexanoate, and dimethyl trisulfur (DMTS) positively contributed to PC1. Phenylalanine, isoleucine, leucine, alanine, aspartic acid, citric acid, glutamic acid, succinic acid, inositol, glycerol, ethanol, and isoamyl acetate were amino acids, organic acids, and other molecules that negatively contributed to PC1. Ethanol, DMTS, phenylalanine, ethyl hexanoate, glycerol, isoleucine, glutamic acid, valine, and threonine negatively contributed to PC2. Serine, succinic acid, malic acid, glucose, urea, isoamyl acetate, aspartic acid, inositol, and alanine positively contributed to PC2. A loading matrix was constructed for the variables (Table 3-S3).

\section{Discussion}

This is the first report of sake brewing by Saccharomyces arboricola and its hybrids. The exploratory metabolic analysis of $S$. arboricola, a neglected Saccharomyces yeast lacking metabolic evaluation, adds to the novelty of this study. Through direct mating of $S$. arboricola to $S$. cerevisiae, interspecific hybrids gained a higher final ethanol content over $S$. cerevisiae and a higher ethyl hexanoate over $S$. arboricola. It was found that the low levels of endogenous amino/organic acids clustered $S$. arboricola apart from the industrial strains by principal component analysis and hierarchical clustering heatmapping. In sake fermentations, hybrid strains showed a mosaic profile of parental strains except where parental profiles were similar. This 
metabolic analysis suggested $S$. arboricola had higher transportation activity of pyruvate to the mitochondria and a lower amino acid net uptake than $S$. cerevisiae. Additionally, hybrid metabolic analysis suggested an increase in ethanolic fermentation from pyruvate and increased sulfur metabolism. Together, these results suggest $S$. arboricola is poised for in-depth and focused metabolomic exploration.

Endogenous metabolite comparison of nine industrially relevant and diverse yeast by GC-MS provided a separation of metabolomic profiles where industry and species appeared to be drivers of distinction in both heat map clustering and PCA. The internal composition is characteristic of their metabolic tendencies and influences the environment in which many endogenous metabolic reactions occur. Of the industrial yeast, sake yeast separated from beer yeast by PC2 of the PCA (Figure 4-1B) which is characterized by the glucose to fructose ratio, inositol level, as well as the organic and amino acid profile. The endogenous inositol levels were high in sake yeast and affect membrane fluidity during osmotic stress such as an industrial sake mash. Sugar utilization played a major role in separation of the strains as evident in the loading plot which highlighted the importance of sugar metabolism. Metabolic distinctions between the glucose to fructose ratio of beer to sake or wine yeasts can be inferred to resemble the kinetic differences of hexokinasemediated sugar phosphorylation. The glucose to fructose ratio between wine strains was correlated with the kinetics of hexokinase-mediated sugar phosphorylation and the inherent properties of each unique strain ${ }^{62,63}$. High amounts of endogenous inositol and trehalose were characteristic of $S$. arboricola. Furthermore, serine and glycine were found to be low in the $S$. arboricola cluster and are important glycolytic metabolites biosynthesized from 3-phosphoglycerate or alternatively through the gluconeogenic pathway utilizing glyoxylate ${ }^{64}$. Organic and amino acid metabolism shift between alternative biosynthesis pathways and their byproducts likely play a role in the difference seen between strains. Additional genetic and environmental factors are also suspected to play a role in strain metabolomic profiles as seen by increased copy number of $M A L$ (maltose) genes in sake and beer yeast, yet wine strains tend to lose this attribute because their typical industrial fermentation sugar availability lacks maltose ${ }^{65}$. Copy number variations (CNV) are concentrated in the sub telomeric regions, with chr. I, VII, VIII, IX, X, XII, XV, and XVI being more susceptible to variation ${ }^{65}$. Gene ontology related to carbon and nitrogen metabolism, ion transport, and flocculation are prone to $\mathrm{CNV}^{66,67}$ which often results in deletions rather than amplifications of genetic material ${ }^{65}$. The CNVs are thought to underline niche adaptation and domestication $^{65,66}$.

Flavor-active elements formed during yeast fermentation can be divided into five key groups: sulfur-containing molecules, organic acids, higher alcohols, carbonyl compounds, and volatile esters $^{68-71}$. Of these classifications, volatile esters are the largest and are responsible for the highly desired fruity character in several alcoholic beverages ${ }^{72}$. Production of ethyl hexanoate and isoamyl alcohol is maximized during fermentations at $15^{\circ} \mathrm{C}^{73,74}$. Ethyl hexanoate imparts apple or aniseed and isoamyl acetate imparts a banana-like attribute to human sensory receptors. Ethyl hexanoate, a MCFA (medium chain fatty acids) ester with a $0.17-0.21 \mathrm{mg} \mathrm{mL}^{-1}$ flavor threshold, is an important flavor molecule for ginjo sake (ginjo-shu, 吟醸酒) ${ }^{68,75}$. The fruity ester, formed from hexanoic acid or hexanoyl CoA with ethanol, imparts a light, sweet, apple complexity to the delicate and fragrant beverage. Ginjo sake is produced from rice with the outer $40 \%$ of the grain polished away to remove the hull, bran, and part of the starchy endosperm, leaving $60 \%$ of the rice to create the sake mash. During the sake fermentation trials, $S$. arboricola produced significantly 
less ethyl hexanoate than either the hybrid or $S$. cerevisiae wine strain M22. Increased inositol concentrations have been shown to decrease production of ethyl hexanoate during fermentation ${ }^{76}$. This relationship was seen in PC1 of the hybrids and suggests $S$. arboricola's inositol biosynthesis pathway is more active, in turn producing low ethyl hexanoate levels seen in this study. Additionally, $S$. arboricola's production of isoamyl acetate showed a trend towards a significant increase over the hybrid or S. cerevisiae M22 (Figure 4-3C). Isoamyl acetate, an acetate ester with a $0.6-1.2 \mathrm{mg} \mathrm{mL}^{-1}$ flavor threshold, imparts a distinct banana character typical of hefeweizen yeast ${ }^{68}$. Hybrid strains produced a pleasant aromatic profile containing both ethyl hexanoate and isoamyl acetate. Ethanol hexanoyl transferase, Eht1, is believed to catalyze the formation of ethyl hexanoate ${ }^{68}$. Alcohol acetyltransferase genes Atf1 and Atf2 are responsible for the total biosynthesis of isoamyl alcohol and divergence in the Atf proteins has been suggested to influence the whole ester aroma profile of yeast strains ${ }^{72}$. Protein identity between $S$. arboricola and $S$. cerevisiae for Atfl was $81 \%$, Atf2 was $36 \%$, and Eht1 was $84 \%$. Together the aromatic ester profile of $S$. arboricola was unique in the combination of low ethyl hexanoate and isoamyl alcohol concentrations are speculated to be owing from inositol lipid activity and variation in factors affecting Atf activity. This area of research is merits further investigation.

Interspecific hybrids are commonly employed in agricultural and horticultural settings where hybrid vigor provides an increased or transgressive phenotype ${ }^{77}$. In oenology Saccharomyces hybrids, such as S. cerevisiae x $S$. kudrazevii, are often employed ${ }^{78,79}$. Many times Saccharomyces hybrids are able to yield more ethanol during fermentation under lab controlled environments ${ }^{80}$, and these results support previous findings. The use of $S$. arboricola in sake production is novel and no other study had $S$. arboricola's metabolism as the primary focus of the manuscript. To date several labs have investigated Saccharomyces hybrids, one study included S. arboricola in a beer brewing context while one other included S. arboricola among their hybrids in a biofuel context ${ }^{80,81}$. This research showed that in a sake mash, SAM interspecific hybrids produced a more vigorous fermentation in a short amount of time which may be beneficial if used or further developed in a production context. Of the range of temperatures for Saccharomyces growth, $S$. arboricola is cryotolerant and thermosensitive $\mathrm{e}^{2,80,82}$. Cryotolerance is a common attribute of yeast hybrids as seen in the lager yeast Saccharomyces pastorianus, which is a hybrid between $S$. cerevisiae x $S$. eubayanus ${ }^{7,83-85}$. This phenotype has proven beneficial for the cold fermentations typical of lagering beer or brewing sake. The ability to ferment at colder temperatures allows for more volatile compounds to be retained and a decrease in bitter or acidic taste ${ }^{86}$. The mechanism by which thermal phenotypes are determined has not been fully elucidated, but mitotype, ploidy, and lipid composition are factors ${ }^{80,86-91}$. Lipid profiles with a higher number of MCFA attributes to cryotolerance of S. cerevisiae $\mathrm{x}$ S. kudriavzevii hybrids $^{92}$. At colder temperatures, increasing the MCFA composition and reducing saturated and unsaturated fatty acid composition allows increased fluidity of the membranes.

Previous reports show that wine yeast produce lower amino acidity and less succinic acid, but more malic acid than sake yeast ${ }^{93}$. Yeast strain has a major impact on metabolic profiles as seen in this study's two wine strains. During fermentation, organic acid production is attributed to the metabolism of pyruvate in the mitochondria and the reverse tricarboxylic acid cycle (TCA) cycle in the cytoplasm ${ }^{94,95}$. Most succinic acid and malic acid are produced through mitochondrial TCA cycle, however reductive TCA can supplement their production during sake fermentation ${ }^{95}$. These amino and organic acidity profiles are metabolically unique to individual strains and these may 
contribute a favorable fresh acidity such as malate while succinate contributes an unfavorable acidity to sake. During sake metabolic analysis, PC1 was inferred to represent the amino acid net uptake and mitochondrial transportation of pyruvate which contributes to the amino and organic acidity profiles (Figure 4-4C). Of the sake metabolites detected, $75 \%$ of the compounds were produced in high abundance in the sake by $S$. arboricola as compared to $S$. cerevisiae which produced $44 \%$ of the compounds in high amounts. S. arboricola was considered to have a lower amino acid net uptake than $S$. cerevisiae strains in this study. The abundance of residual amino acids detected in the sake medium and the low abundance of endogenous metabolites were considered in these findings. Moreover, membrane fluidity and amino acid and organic acid transport of S. arboricola has not been investigated and merits further research.

The generated interspecific hybrids were considered to have vigorous glycolysis and fermentation of pyruvate to ethanol compared to parental strains. The reduced malic acid, succinic acid, and glucose (Figure 4-4A) compared to the increased ethanol yield (Figure 4-3A) supported these claims. The hybrid condition was distinctly exemplified in principal component 2 of the sake metabolite score plot. In this study, where parental metabolic profiles were similar, the hybrids revealed an inversion of these compounds in the heatmap as seen in DMTS, serine, urea, and succinic acid. DMTS is associated with the stale aroma of Japanese sake called hineka (老香). Recycling of sulfur via the methionine salvage pathway produces DMTS-P1, a precursor of this aromatic compound ${ }^{96}$. Mercaptans (thiols) typically have a low sensory threshold and the hybrids were considered to have an active sulfur metabolism. Additionally, urea is a yeast waste product from arginase activity and can be catabolized to form succinate in the cell $1{ }^{97}$. Mechanistic elucidation of these metabolic variances is beyond the scope of this initial report of sake fermentation by $S$. arboricola and the hybrids, further research is required.

Research for $S$. arboricola yeast is very limited and one other report ${ }^{80}$, to the author's knowledge, has explored their use and their interspecific hybrids' use in beverage fermentation. During their wort fermentation trials, $S$. arboricola produced high levels of ethyl hexanoate at $0.35 \mathrm{mg} \mathrm{mL}^{-1}$, while this study revealed low production at $0.207 \mathrm{mg} \mathrm{mL}^{-1}$ by substituting sake mash for wort. Yeast strain and raw ingredients certainly play a role in the final fermentation product qualities. The chemistry of sake and beer have some similarities, but there are also many differences including raw materials such as rice or barley and the presence of A. oryzae in the sake mash. These results indicate koji was an integral part of the sake mash converting rice carbohydrate into fermentable sugars. Aspergillus spp. produce mannitol at high levels ${ }^{46,47}$ and $S$. arboricola has great growth utilizing mannito ${ }^{14}$, but the bioavailability of this sugar for fermentation by $S$. arboricola remains unexplored. This metabolic capability likely facilitated the increase in fermentation performance seen in this study. S. arboricola and their hybrids may be uniquely fit for use in sake fermentations.

In two recent large phylogenetic analysis of 157 and 1,011 Saccharomyces cerevisiae strains, an Asian cluster was found basal to the root of the trees ${ }^{34,65}$ suggesting little divergence from their wild ancestors in comparison to the highly diverged beerl clade. Although 1,011 genome study was a great step in unmasking the lost ecology and domestication of natural Saccharomyces yeast, it had an overrepresentation of beer brewing strains. In order to dive deeper into the Out of Asia Origin Hypothesis, many more Asian and diverse strains will need to be sequenced and compared phylogenetically. The investigation into $S$. arboricola's biotechnological value yielded promising 
results, but the dynamics between $A$. oryzae's mannitol production and $S$. arboricola's mannitol utilization merits further research. Exploration of the biotechnical potential of the Saccharomyces clade is only now beginning and understanding the diversity of yeast facilitates humanity's exploitation of Saccharomyces.

\section{Conclusions}

This study explored the novel use of $S$. arboricola in Japanese rice wine known as sake, traditionally produced from polished rice with $A$. oryzae during winter months. The metabolites in the sake produced from $S$. arboricola, $S$. cerevisiae, and their interspecific hybrids were distinct by PCA analysis. $S$. arboricola had a lower amino acid net uptake than $S$. cerevisiae and the hybrids showed a mosaic profile of parental strains. Hybrid strains had a more vigorous ethanolic fermentation in the sake mash than $S$. cerevisiae but had a higher ethyl hexanoate yield than $S$. arboricola. In the ginjo style of Japanese sake, ethanol and ethyl hexanoate are important sensory metabolic compounds. S. arboricola differed from $S$. cerevisiae industrial strains because of lower levels of endogenous amino/organic acids, suggesting a lower amino acid net uptake. The endogenous metabolite study highlighted the impact of strain selection which industry niche has unwittingly had an influence in each strains metabolism. The diversity of the Saccharomyces clade of budding yeast is expansive and future studies focused metabolic phenotypes of new strains and novel applications will most likely surface in the following years to come.

Acknowledgements: Thank you Jenny Bellon from the Austrian Wine Research Institute who aided with experimental design, Gianni Liti of Institute for Research on Cancer and Aging (IRCAN) who provided the Saccharomyces arboricola strains, Barbra Dunn of Stanford University who provided advice and materials, Angela Chu/Joe Horecka of Stanford University who shared their sporulation protocol, and Tetsuya Goshima/Takeshi Akao of the Japanese National Research Institute of Brewing (NRIB) who provided access to the micromanipulator and materials/provided the $\mathrm{K} 7 / 868$ haploid.

Contribution: Conceptualization, M.J.W., J.E.G.G., and H.K.; Methodology, M.J.W.; Validation, M.J.W., Y.Y., and Y.F.; Formal Analysis, M.J.W., Y.Y., and Y.F.; Investigation, M.J.W., Y.F., and Y.Y.; Resources, H.K., Y.Y., Y.F., and Y.K.; Data Curation, Y.Y., Y.F., and Y.K.; Writing Original Draft Preparation, M.J.W.; Writing - Review \& Editing, M.J.W., J.E.G.G., and H.K.; Visualization, M.J.W., Y.F., and Y.Y.; Supervision, H.K.; Project Administration, M.J.W. and H.K.; Funding Acquisition, M.J.W and H.K.

Funding: This material is based upon work supported by the National Science Foundation's East Asia and Pacific Summer Institute Program [Grant No. 1713160], in conjunction with the Japanese Society for the Promotion of Science Summer Program ID:SP17053 to MJW. 


\section{References}

1. Martini, A. V. \& Martini, A. Three newly delimited species of Saccharomyces sensu stricto. Antonie Van Leeuwenhoek 53, 77-84 (1987).

2. Wang, S. A. \& Bai, F. Y. Saccharomyces arboricolus sp. nov., a yeast species from tree bark. Int. J. Syst. Evol. Microbiol. 58, 510-514 (2008).

3. Naumova, E. S., Roberts, I. N., James, S. A., Naumov, G. I. \& Louis, E. J. Three new species in the Saccharomyces sensu stricto complex: Saccharomyces cariocanus, Saccharomyces kudriavzevii and Saccharomyces mikatae. Int. J. Syst. Evol. Microbiol. 50, 1931-1942 (2015).

4. Naumov, G. I., Naumova, E. S., Hagler, A. N., Mendonça-Hagler, L. C. \& Louis, E. J. A new genetically isolated population of the Saccharomyces sensu stricto complex from Brazil. Antonie Van Leeuwenhoek 67, 351-355 (1995).

5. Naumov, G. I., Lee, C.-F. \& Naumova, E. S. Molecular genetic diversity of the Saccharomyces yeasts in Taiwan: Saccharomyces arboricola, Saccharomyces cerevisiae and Saccharomyces kudriavzevii. Antonie Van Leeuwenhoek 103, 217-28 (2013).

6. Naumov, G. I., Naumova, E. S. \& Louis, E. J. Two New Genetically Isolated Popoulations of the Saccharomyces Sensu Stricto Complex from Japan. J. Gen. Appl. Microbiol. 41, 499505 (1995).

7. Libkind, D. et al. Microbe domestication and the identification of the wild genetic stock of lager-brewing yeast. Proc. Natl. Acad. Sci. U. S. A. 108, 14539-44 (2011).

8. Boynton, P. J. \& Greig, D. The ecology and evolution of non-domesticated Saccharomyces species Primrose. Yeast 31, 449-462 (2014).

9. Sipiczki, M. Interspecies hybridisation and genome chimerisation in Saccharomyces: Combining of gene pools of species and its biotechnological perspectives. Front. Microbiol. 9, 1-20 (2018).

10. Masneuf, I., Hansen, J., Groth, C., Piskur, J. \& Dubourdieu, D. New hybrids between Saccharomyces sensu stricto yeast species found among wine and cider production strains. Appl. Environ. Microbiol. 64, 3887-3892 (1998).

11. Querol, A. \& Bond, U. The complex and dynamic genomes of industrial yeasts: MINIREVIEW. FEMS Microbiol. Lett. 293, 1-10 (2009).

12. Nguyen, H. V., Legras, J. L., Neuvéglise, C. \& Gaillardin, C. Deciphering the hybridisation history leading to the lager lineage based on the mosaic genomes of Saccharomyces bayanus strains NBRC1948 and CBS380 T. PLoS One 6, (2011).

13. Gayevskiy, V.\& Goddard, M. R. Saccharomyces eubayanus and Saccharomyces arboricola reside in North Island native New Zealand forests. Environ. Microbiol. 18, 1137-1147 (2016).

14. Liti, G. et al. High quality de novo sequencing and assembly of the Saccharomyces arboricolus genome. BMC Genomics 14, 14:69 (2013).

15. Cliften, P. et al. Finding functional features in Saccharomyces genomes by phylogenetic footprinting. Science (80-. ). 301, 71-76 (2003).

16. Birren, B., Lander, E. S., Kellis, M., Endrizzi, M. \& Patterson, N. Sequencing and comparison of yeast species to identify genes and regulatory elements. Nature 423, 241254 (2003).

17. Liti, G. et al. Population genomics of domestic and wild yeasts. Nature 458, 337-41 (2009).

18. Dujon, B. Yeasts illustrate the molecular mechanisms of eukaryotic genome evolution. 
Trends Genet. 22, 375-387 (2006).

19. Postma, E., Verduyn, C., Scheffers, A. \& Van Dijken, johannes P. Enzymic analysis of the Crabtree Effect in glucose-limited chemostat cultures of Saccharomyces cerevisiae. Appl. Environ. Microbiol. 55, 468-477 (1989).

20. Galdieri, L., Mehrotra, S., Yu, S. \& Vancura, A. Transcriptional regulation in yeast during diauxic shift and stationary phase. Omi. A J. Integr. Biol. 14, 629-638 (2010).

21. McGovern, P. E. et al. Fermented beverages of pre- and proto-historic China. Proc. Natl. Acad. Sci. U. S. A. 101, 17593-8 (2004).

22. McGovern, P. E. Ancient Wine: The Search for the Origins of Viniculture. Ancient Wine (Princeton Publishing, 2003). doi:10.1663/0013-0001(2004)058[0488:DFABRE]2.0.CO;2

23. Kitagaki, H. \& Kitamoto, K. Breeding Researches of Sake Yeasts in Japan: History, Recent Technological Advances, and Future Perspectives. ... Food Sci. Technol. 4, 1-21 (2012).

24. Charles, M. et al. Sixty million years in evolution of soft grain trait in grasses: Emergence of the softness locus in the common ancestor of pooideae and ehrhartoideae, after their divergence from panicoideae. Mol. Biol. Evol. 26, 1651-1661 (2009).

25. Arendt, E. \& Zannini, E. Rice. in Cereal grains for the food and beverage industries (Woodhead Publishing, 2013).

26. Corran, H. S. A history of brewing. (David \& Charles, 1975).

27. Sampaio, J. P. Microbe profile: Saccharomyces eubayanus, the missing link to lager beer yeasts. Microbiol. (United Kingdom) 164, 1069-1071 (2018).

28. Peris, D. et al. Complex Ancestries of Lager-Brewing Hybrids Were Shaped by Standing Variation in the Wild Yeast Saccharomyces eubayanus. PLoS Genet. 12, 1-20 (2016).

29. Nespolo, R. F. et al. An Out-of-Patagonia dispersal explains most of the worldwide genetic distribution in Saccharomyces eubayanus. bioRxiv 709253 (2019). doi:10.1101/709253

30. Langdon, Q. K. et al. Genomic diversity and global distribution of Saccharomyces eubayanus, the wild ancestor of hybrid lager-brewing yeasts. bioRxiv 709535 (2019). doi:10.1101/709535

31. Eizaguirre, J. I. et al. Phylogeography of the wild Lager-brewing ancestor (Saccharomyces eubayanus) in Patagonia. Environ. Microbiol. 20, 3732-3743 (2018).

32. Nagy, L. G. et al. Latent homology and convergent regulatory evolution underlies the repeated emergence of yeasts. Nat. Commun. 5, (2014).

33. Bing, J., Han, P. J., Liu, W. Q., Wang, Q. M. \& Bai, F. Y. Evidence for a far east asian origin of lager beer yeast. Curr. Biol. 24, R380-R381 (2014).

34. Peter, J. et al. Genome evolution across 1,011 Saccharomyces cerevisiae isolates. Nature 556, 339-344 (2018).

35. Duan, S. F. et al. The origin and adaptive evolution of domesticated populations of yeast from Far East Asia. Nat. Commun. 9, (2018).

36. He, Y. et al. Wort composition and its impact on the fl avour-active higher alcohol and ester formation of beer - a review. 157-163 (2014). doi:10.1002/jib.145

37. Engan, S. Wort Composition and Beer Flavour. II. The Influence of Different Carbohydrates on the Formation of Some Flavour Components During Fermentation. J. Inst. Brew. 78, 169-173 (1971).

38. Younis, O. S. \& Stewart, G. G. Sugar uptake and subsequent ester and higher alcohol production by Saccharomyces cerevisiae. J. Inst. Brew. 104, 255-264 (1998).

39. Mortimer, R. \& Polsinelli, M. On the origins of wine yeast. Res. Microbiol. 150, 199-204 (1999). 
40. Bisson, L. F. Gene Expression in Yeasts During Wine Fermentation. in Yeasts in The Production of Wine (eds. P., R., M., C. \& G., F.) 165-209 (Springer, 2019).

41. Kühbeck, F., Back, W. \& Krottenthaler, M. Influence of lauter turbidity on wort composition, fermentation performance and beer quality - A review. J. Inst. Brew. 112, 215-221 (2006).

42. White, C. \& Zainasheff, J. Yeast: The Practical Guide to Beer Fermentation (Brewing Elements). (Brewers Publications, 2010).

43. Kodama, K. Sake Yeast. in The Yeasts (eds. H, R. \& J. S., H.) 129-168 (Academic Press, 1993).

44. Ferdouse, J. et al. Glycosylceramide modifies the flavor and metabolic characteristics of sake yeast. PeerJ 2018, 1-16 (2018).

45. Zhang, B., Guan, Z. B., Cao, Y., Xie, G. F. \& Lu, J. Secretome of Aspergillus oryzae in Shaoxing rice wine koji. Int. J. Food Microbiol. 155, 113-119 (2012).

46. Song, S. H. \& Vieille, C. Recent advances in the biological production of mannitol. Appl. Microbiol. Biotechnol. 84, 55-62 (2009).

47. Smiley, K. L., Cadmus, M. C. \& Liepins, P. Biosynthesis of D-mannitol from D-glucose by Aspergillus candidus. Biotechnol. Bioeng. 9, 365-374 (1967).

48. Bellon, J. R., Schmid, F., Capone, D. L., Dunn, B. L. \& Chambers, P. J. Introducing a New Breed of Wine Yeast: Interspecific Hybridisation between a Commercial Saccharomyces cerevisiae Wine Yeast and Saccharomyces mikatae. PLoS One 8, (2013).

49. González, S. S., Barrio, E., Gafner, J. \& Querol, A. Natural hybrids from Saccharomyces cerevisiae, Saccharomyces bayanus and Saccharomyces kudriavzevii in wine fermentations. FEMS Yeast Res. 6, 1221-1234 (2006).

50. Capece, A., Romaniello, R., Siesto, G. \& Romano, P. Diversity of Saccharomyces cerevisiae yeasts associated to spontaneously fermenting grapes from an Italian "heroic vine-growing area." Food Microbiol. 31, 159-166 (2012).

51. Song, G. et al. AGAPE (Automated Genome Analysis PipelinE) for pan-genome analysis of Saccharomyces cerevisiae. PLoS One 10, 1-19 (2015).

52. Katou, T., Kitagaki, H., Akao, T. \& Shimoi, H. Brewing characteristics of haploid strains isolated from sake yeast Kyokai No. 7. Yeast 25, 799-807 (2008).

53. Horie, K. et al. Breeding of a Low Pyruvate-Producing Sake Yeast by Isolation of a Mutant Resistant to Ethyl $\alpha$-Transcyanocinnamate, an Inhibitor of Mitochondrial Pyruvate Transport. Biosci. Biotechnol. Biochem. 74, 843-847 (2010).

54. Sniegowski, P. D., Dombrowski, P. G. \& Fingerman, E. Saccharomyces cerevisiae and Saccharomyces paradoxus coexist in a natural woodland site in North America and display different levels of reproductive isolation from European conspecifics. FEMS Yeast Res. 1, 299-306 (2002).

55. Schoch, C. L. et al. Nuclear ribosomal internal transcribed spacer (ITS) region as a universal DNA barcode marker for Fungi. Proc. Natl. Acad. Sci. U. S. A. 109, 1-6 (2012).

56. Rong-Mullins, X. et al. Proteomic and genetic analysis of the response of S. cerevisiae to soluble copper leads to improvement of the antimicrobial function of cellulosic copper nanoparticles. Metallomics 9, 1304-1315 (2017).

57. Shimwell, J. L. a Simple Staining Method for the Detection of Ascospores in Yeasts. J. Inst. Brew. 44, 474-474 (1938).

58. Muir, A., Harrison, E. \& Wheals, A. A multiplex set of species-specific primers for rapid identification of members of the genus Saccharomyces. FEMS Yeast Res. 11, 552-563 
(2011).

59. Korbie, D. J. \& Mattick, J. S. Touchdown PCR for increased specificity and sensitivity in PCR amplification. Nat. Protoc. 3, 1452-1456 (2008).

60. Lee, P. Y., Costumbrado, J., Hsu, C.-Y. \& Kim, Y. H. Agarose Gel Electrophoresis for the Separation of DNA Fragments. J. Vis. Exp. 1-5 (2012). doi:10.3791/3923

61. Xia, J., Sinelnikov, I. V., Han, B. \& Wishart, D. S. MetaboAnalyst 3.0-making metabolomics more meaningful. Nucleic Acids Res. 43, W251-W257 (2015).

62. Berthels, N. J., Cordero Otero, R. R., Bauer, F. F., Thevelein, J. M. \& Pretorius, I. S. Discrepancy in glucose and fructose utilisation during fermentation by Saccharomyces cerevisiae wine yeast strains. FEMS Yeast Res. 4, 683-689 (2004).

63. Berthels, N. J., Cordero Otero, R. R., Bauer, F. F., Pretorius, I. S. \& Thevelein, J. M. Correlation between glucose/fructose discrepancy and hexokinase kinetic properties in different Saccharomyces cerevisiae wine yeast strains. Appl. Microbiol. Biotechnol. 77, 1083-1091 (2008).

64. Melcher, K. \& Entian, K.-D. Genetic analysis of serine biosynthesis and glucose repression in yeast. Curr. Genet. 21, 295-300 (1992).

65. Gallone, B. et al. Domestication and Divergence of Saccharomyces cerevisiae Beer Yeasts. Cell 166, 1397-1410.e16 (2016).

66. Dunn, B., Richter, C., Kvitek, D. J., Pugh, T. \& Sherlock, G. Analysis of the Saccharomyces cerevisiae pan-genome reveals a pool of copy number variants distributed in diverse yeast strains from differing industrial environments. Genome Res. 22, 908-924 (2012).

67. Bergström, A. et al. A high-definition view of functional genetic variation from natural yeast genomes. Mol. Biol. Evol. 31, 872-888 (2014).

68. Verstrepen, K. J. et al. Flavor-active esters: Adding fruitiness to beer. J. Biosci. Bioeng. 96, 110-118 (2003).

69. Pisarnitskii, A. F. Formation of wine aroma: Tones and imperfections caused by minor components (review). Appl. Biochem. Microbiol. 37, 552-560 (2001).

70. Pretorius, I. S. \& Lambrechts, M. G. Yeast and its importance to wine aroma: a review. South African J. Enol. Vitic. 21, 97-129 (2000).

71. Quilter, M. G., Hurley, J. C., Lynch, F. J. \& Murphy, M. G. The Production of Isoamyl Acetate from Amyl Alcohol by Saccharomyces cerevisiae. J. Inst. Brew. 109, 34-40 (2003).

72. Verstrepen, K. J. et al. Expression Levels of the Yeast Alcohol Acetyltransferase Genes ATF1, Lg-ATF1, and ATF2 Control the Formation of a Broad Range of Volatile Esters. Appl. Environ. Microbiol. 69, 5228-5237 (2003).

73. Norstedt, C., Bengtsson, A., Bennet, P., Lindström, I. \& Äyräpää, T. Technical Measures to Control the Formation of Esters During Beer Fermentation. in European Brewery Convention: Proceedings of the 15th Congress (ed. Nice) 581-600 (Elsevier Scientific Publishing Company, 1975).

74. Hammond, J. R. M. Yeast Technology. in Brewer's Yeast (eds. Rose, A. H. \& Harrison, J. S.) 5, 8-56 (Academic Press, 1993).

75. Takahashi, T., Ohra, Y.\& Sueno, K. Breeding of a sake yeast mutant with enhanced ethyl caproate productivity in sake brewing using rice milled at a high polishing ratio. J. Biosci. Bioeng. 123, 707-713 (2017).

76. Furukawa, K., Yamada, T., Mizoguchi, H. \& Hara, S. Increased Ethyl Caproate Production by Inositol Limitation in Saccharomyces cerevisiae. J. BIOSCENCE Bioeng. 95, 448-454 (2003). 
77. Dittrich-Reed, D. R. \& Fitzpatrick, B. M. Transgressive Hybrids as Hopeful Monsters. Evol. Biol. 40, 310-315 (2013).

78. Bisson, L. F. Yeast Hybrids in Winemaking. Catal. Discov. into Pract. 1, 27-34 (2017).

79. Morales, L. \& Dujon, B. Evolutionary Role of Interspecies Hybridization and Genetic Exchanges in Yeasts. Microbiol. Mol. Biol. Rev. 76, 721-739 (2012).

80. Nikulin, J., Krogerus, K. \& Gibson, B. Alternative Saccharomyces interspecies hybrid combinations and their potential for low-temperature wort fermentation. Yeast 35, 113-127 (2018).

81. Peris, D. et al. Hybridization and adaptive evolution of diverse Saccharomyces species for cellulosic biofuel production. Biotechnol. Biofuels 10, 1-19 (2017).

82. Salvadó, Z. et al. Temperature adaptation Markedly Determines evolution within the genus Saccharomyces. Appl. Environ. Microbiol. 77, 2292-2302 (2011).

83. Krogerus, K., Magalhes, F., Vidgren, V. \& Gibson, B. New lager yeast strains generated by interspecific hybridization. J. Ind. Microbiol. Biotechnol. 769-778 (2015). doi:10.1007/s10295-015-1597-6

84. Magalhães, F., Krogerus, K., Vidgren, V., Sandell, M. \& Gibson, B. Improved cider fermentation performance and quality with newly generated Saccharomyces cerevisiae $\times$ Saccharomyces eubayanus hybrids. J. Ind. Microbiol. Biotechnol. 44, 12031213 (2017).

85. Magalhães, F. et al. Exploring the potential of Saccharomyces eubayanus as a parent for new interspecies hybrid strains in winemaking. FEMS Yeast Res. 17, 1-10 (2017).

86. Torija, M. J. et al. Effects of fermentation temperature and Saccharomyces species on the cell fatty acid composition and presence of volatile compounds in wine. Int. J. Food Microbiol. 85, 127-136 (2003).

87. Krogerus, K., Magalhães, F., Vidgren, V. \& Gibson, B. Novel brewing yeast hybrids: creation and application. Appl. Microbiol. Biotechnol. 101, 65-78 (2017).

88. Fay, J. C., Hittinger, C. T., Sia, E. A., Peris, D. \& Li, X. C. Mitochondria-encoded genes contribute to evolution of heat and cold tolerance in yeast. Sci. Adv. 5, eaav1848 (2019).

89. Beltran, G., Novo, M., Guillamón, J. M., Mas, A. \& Rozès, N. Effect of fermentation temperature and culture media on the yeast lipid composition and wine volatile compounds. Int. J. Food Microbiol. 121, 169-177 (2008).

90. Redón, M., Guillamón, J. M., Mas, A. \& Rozès, N. Effect of lipid supplementation upon Saccharomyces cerevisiae lipid composition and fermentation performance at low temperature. Eur. Food Res. Technol. 228, 833-840 (2009).

91. Baker, E. P. et al. Mitochondrial DNA and temperature tolerance in lager yeasts. Sci. Adv. 5, eaav1869 (2019).

92. Tronchoni, J., Rozès, N., Querol, A. \& Guillamón, J. M. Lipid composition of wine strains of Saccharomyces kudriavzevii and Saccharomyces cerevisiae grown at low temperature. Int. J. Food Microbiol. 155, 191-198 (2012).

93. Iwase, T. et al. Breeding of Sake Yeast by Protoplast Fusion of Sake Yeast and Wine Yeast. J. Brew. Soc. Japan 90, 137-142 (1995).

94. Motomura, S., Horie, K. \& Kitagaki, H. Mitochondrial activity of sake brewery yeast affects malic and succinic acid production during alcoholic fermentation. J. Inst. Brew. 118, 22-26 (2012).

95. Asano, T., Kurose, N., Hiraoka, N. \& Kawakita, S. Effect of NAD+-dependent isocitrate dehydrogenase gene (IDH1, IDH2) disruption of sake yeast on organic acid composition in 
sake mash. J. Biosci. Bioeng. 88, 258-263 (1999).

96. Wakabayashi, K., Isogai, A., Watanabe, D., Fujita, A. \& Sudo, S. Involvemwnt of methionine salvage pathway genes of Saccharomyces cerevisiae in the production of precursor compounds of dimethyl trisulfide (DMTS). J. Biosci. Bioeng. 116, 475-479 (2013).

97. Schneider, B. L., Kiupakis, A. K. \& Reitzer, L. J. Arginine catabolism and the arginine succinyltransferase pathway in Escherichia coli. J. Bacteriol. 180, 4278-4286 (1998). 


\section{Appendix C}

\section{Supplemental Figures}

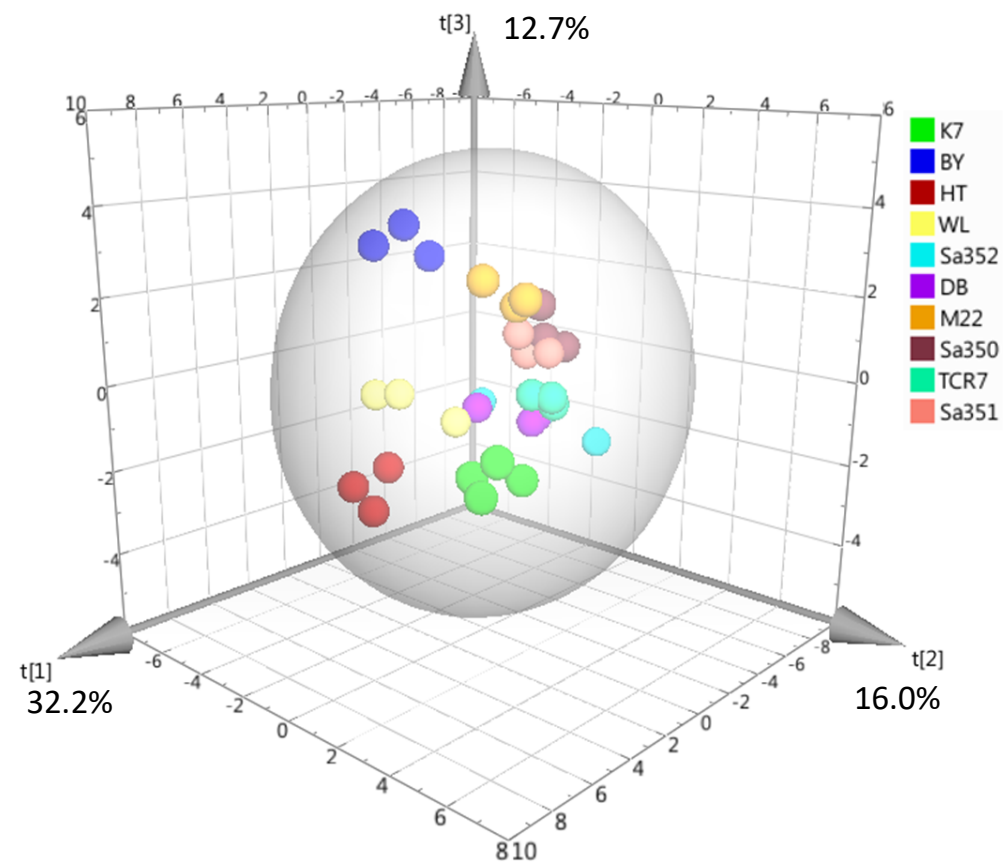

Ellipse: Hotelling's T2 (95\%)

Figure 4-S1. 3D PCA Score Plot of the Saccharomyces Yeast Endogenous Metabolites. The PCA plot has three PCs representing $32.2 \%, 16 \%$, and $12.7 \%$ of the sample variances. Yeast strains are labeled by color. 
S. cerevisiae $\mathrm{M} 22$

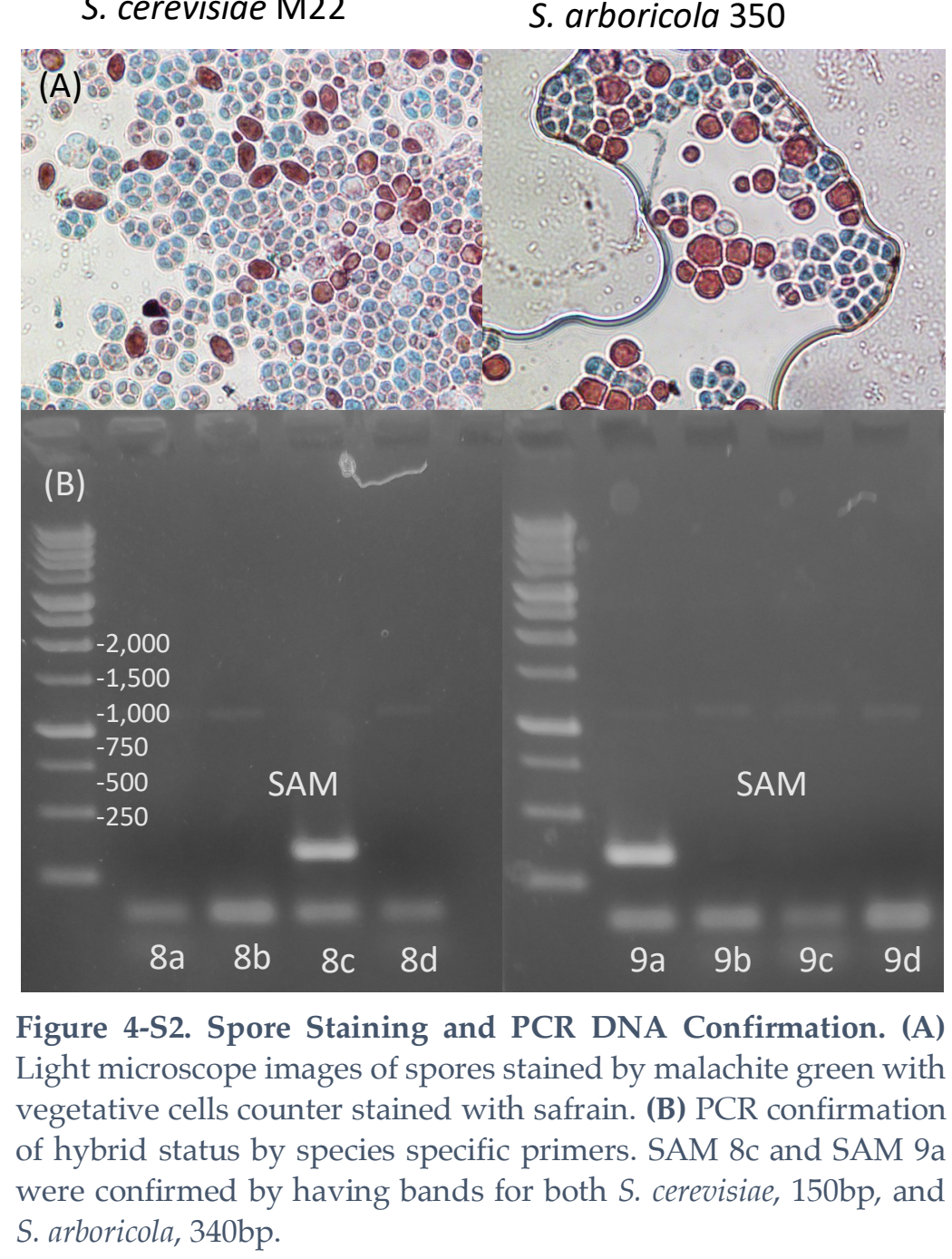

Figure 4-S2. Spore Staining and PCR DNA Confirmation. (A) Light microscope images of spores stained by malachite green with vegetative cells counter stained with safrain. (B) PCR confirmation of hybrid status by species specific primers. SAM 8c and SAM 9a were confirmed by having bands for both S. cerevisiae, 150bp, and S. arboricola, 340bp.

S. arboricola 350 

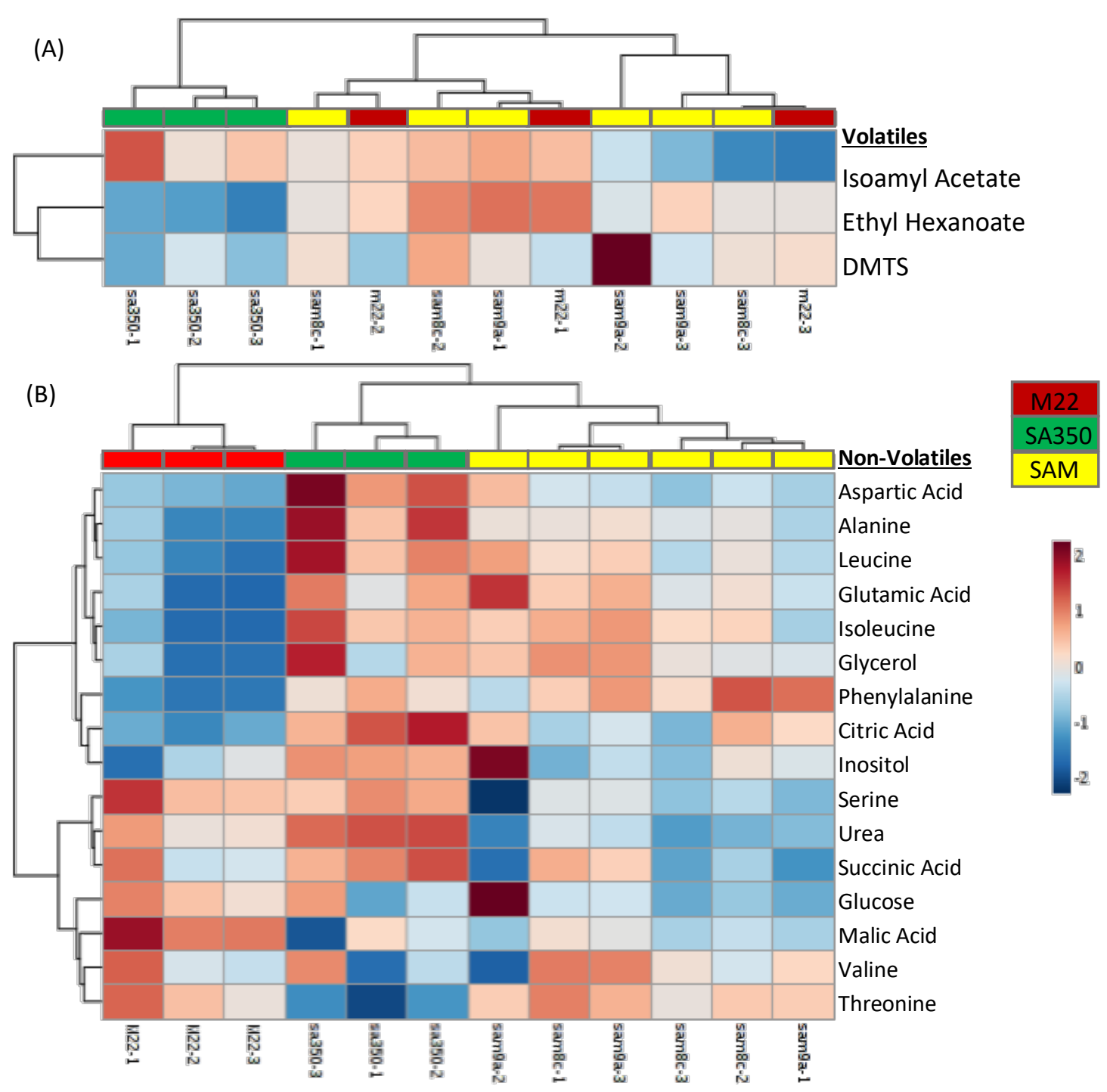

Figure 4-S3. Sake Fermentation Metabolic Profile of S. arboricola Interspecific Hybrids and Parental Strains Sample by Sample. (A) Hierarchical clustering heatmap of sake fermentation volatile profiles between strains and species. (B) Hierarchical clustering heatmap of sake fermentation non-volatile profiles between strains and species. Relatively high compound concentrations were shown in varying degrees of red. Relatively low compound concentrations were shown in varying degrees of blue. Shown as individual sample concentrations of three biological replicates each. 


\section{Supplemental Tables}

Table 4-S1. Endogenous Metabolite Loading Matrix.

\begin{tabular}{|c|c|c|}
\hline $\begin{array}{l}\text { Endogenous } \\
\text { Metabolite }\end{array}$ & M1.p[1] & M1.p[2] \\
\hline serine1 & 0.298183 & 0.151295 \\
\hline threonine 1 & 0.270912 & 0.24956 \\
\hline nicotinic & 0.084131 & 0.304491 \\
\hline glycine & 0.164934 & 0.323333 \\
\hline succinic & 0.046036 & 0.125748 \\
\hline uracil & 0.150956 & -0.29857 \\
\hline fumaric & 0.296116 & 0.167411 \\
\hline serine 2 & 0.307159 & 0.029229 \\
\hline threonine 2 & 0.295011 & 0.035795 \\
\hline methionine1 & 0.209894 & -0.26806 \\
\hline aspartic1 & 0.243726 & -0.16329 \\
\hline malic & 0.258265 & 0.198813 \\
\hline aspartic2 & 0.265834 & -0.28568 \\
\hline pyroglutamic & 0.249486 & -0.30179 \\
\hline phenylalanine 1 & 0.187078 & -0.24547 \\
\hline glutamic acid2 & 0.171301 & 0.008042 \\
\hline citric & 0.270136 & -0.03397 \\
\hline adenine1 & 0.134758 & -0.00263 \\
\hline glucose1 & -0.1288 & -0.25644 \\
\hline glucose 2 & -0.13053 & -0.21503 \\
\hline m-inositol & 0.076818 & -0.02 \\
\hline sucrose & -0.05538 & 0.249083 \\
\hline trehalose & -0.0911 & 0.189166 \\
\hline
\end{tabular}


Table 4-S2. Connecting Letters Report of Significance for Sake Fermentations. Samples that have matching letters are not significant, samples that do not have the same letter are significant $($ alpha $=0.05)$.

\begin{tabular}{|c|c|c|c|}
\hline Day & \multicolumn{1}{c}{ S. c } & \multicolumn{1}{c}{ S. $\boldsymbol{a}$} & Hybrid \\
\hline 1 & A & A & A \\
2 & A & A & A \\
3 & A & A & A \\
4 & A & A & A \\
\hline 5 & A & A & A \\
6 & A & A & A \\
7 & A & A,B & B \\
\hline 8 & A & A,B & B \\
\hline 9 & A & A,B & B \\
\hline 10 & A & A,B & B \\
\hline 11 & A & A,B & B \\
\hline 12 & A & A,B & B \\
\hline 13 & A & A,B & B \\
\hline 14 & A & A,B & B \\
\hline
\end{tabular}


Table 4-S3. Sake Metabolite Loading Matrix

\begin{tabular}{|l|c|c|}
\hline Sake Metabolite & M1.p[1] & M1.p[2] \\
\hline valine & 0.039308 & -0.03906 \\
\hline alanine & -0.32672 & 0.026332 \\
\hline leucine & -0.32873 & 0.000774 \\
\hline isoleucine & -0.29454 & -0.11937 \\
\hline urea & -0.0258 & 0.28709 \\
\hline glycerol & -0.26263 & -0.12227 \\
\hline succinic acid & -0.15761 & 0.335353 \\
\hline threonine & 0.253427 & -0.0692 \\
\hline aspartic acid & -0.31879 & 0.115173 \\
\hline malic acid & 0.246756 & 0.290595 \\
\hline glutamic acid & -0.28849 & -0.11192 \\
\hline serine-3TMS & 0.00122 & 0.4507 \\
\hline phenylalanine & -0.16184 & -0.3145 \\
\hline citric acid & -0.28593 & 0.003946 \\
\hline glucose & 0.046795 & 0.311003 \\
\hline inositol & -0.27947 & 0.047063 \\
\hline isoamylacetate & -0.12037 & 0.165932 \\
\hline ethyl caproate & 0.268673 & -0.20688 \\
\hline DMTS & 0.121216 & -0.31587 \\
\hline ethanol & -0.05329 & -0.29592 \\
\hline
\end{tabular}




\section{CHAPTER 5}

\section{General Conclusions}

Since the first electron microscopes provided nanometer resolution in the early $20^{\text {th }}$ century, the field of nanotechnology has grown considerably. Early understanding of nanotechnology was limited to artists who unwittingly used metal nanoparticles to construct the beautiful stained glass seen in medieval cathedrals and the dichroic glass seen in the Roman Lycurgus Cup ${ }^{1,2}$. Although there is still much to be discovered in material science, there is a push to gain a deeper understanding of how these novel materials interact with biology. Shrinking materials to the nanoscale often gives the nanomaterials unique properties compared to the bulk materials, such as solid metals. Gold, for instance, is known for its scalable and size-dependent surface atom availability that determines its color $^{3,4}$. Nanocopper is less studied and less utilized than other malleable precious metals such as gold or silver ${ }^{5}$. Even less studied are the hybrid cellulose copper nanomaterials such as the novel $\mathrm{CMC}-\mathrm{Cu}$ investigated within this dissertation. The four progressive levels of understanding nanotoxicity include the particle characteristics, cellular toxicity, organ toxicity, and in vivo toxicity ${ }^{6}$. Chapters Two and Three of this dissertation cover the cellular toxicity of $\mathrm{CMC}-\mathrm{Cu}$ nanoparticles.

This dissertation's chapters are arranged to illustrate an emphasis on progressive movement toward dynamic measurements. The risk to consumers of nanotechnology and consumers of fermented beverages is this dissertation's overarching focus. $\mathrm{CMC}-\mathrm{Cu}$ nanotechnology was explored using genetics and proteomics in Chapter Two while the metallomics and lipidomics were investigated in Chapter Three. In Chapter Four, the metabolomics of a novel species was characterized and described. S. arboricola has received interest from the fermented beverage industry and has yet to receive the generally recognized as safe (GRAS) status by the United States Food and Drug Administration (FDA).

Although $S$. cerevisiae's response to copper had been well characterized, novel nanomaterials like $\mathrm{CMC}-\mathrm{Cu}$ remained toxicologically uncharacterized. This novel material was known to be toxic to E. coli $^{7}$ and to further characterize the toxicity of $\mathrm{CMC}-\mathrm{Cu}$, we investigated the genetic and proteomic response of genetically diverse yeast. Chapter Two showed variation in the sensitivity and resistance to $\mathrm{CMC}-\mathrm{Cu}$ that mirrored soluble copper. A QTL analysis revealed the contribution of Zrt transporters in displaying a resistant phenotype upon exposure to soluble copper. Additionally, the addition of zinc back into the media slightly rescued growth, supporting a role in this metal's influence on $\mathrm{Cu}$ toxicity. Considerable amounts of $\mathrm{CMC}-\mathrm{Cu}$ were found to be located on the outside of the cell wall by flow cytometry and fluorescent microscopy experiments ${ }^{8}$; but the rescue of $\mathrm{CMC}-\mathrm{Cu}$ toxicity from arrestin knockouts suggested that some endocytosis into the cell occurred. Hence, in Chapter Two, we identified the unexplored toxicity of $\mathrm{CMC}-\mathrm{Cu}$, and the mechanisms by which $\mathrm{CMC}-\mathrm{Cu}$ becomes associated with the yeast cells.

In the third chapter, I expanded the detail of the nanotoxicological mechanisms and consequences of $\mathrm{CMC}-\mathrm{Cu}$ exposure. Further exploration into the copper, iron, and zinc metal transporters that were upregulated in response to copper identified the unbalanced copper - zinc homeostasis. In response to $\mathrm{CMC}-\mathrm{Cu}$, the sensitive strain significantly depleted zinc stores suggesting a possible 
ionic mimicry relationship, a trait not found in the resistant strain. Copper abundance increased, zinc decreased, and iron had no significance in $\mathrm{CMC}-\mathrm{Cu}$ sensitivity. Considering this imbalance and the ionic/molecular mimicry mechanism of other metal intoxications ${ }^{9,10}$, it is likely that CMC$\mathrm{Cu}$ participates in this mechanism by mimicking $\mathrm{Zn}$ for import. The slight rescue of copper toxicity by the addition of zinc to the media is supportive of this finding, but without fully rescuing the toxicity it is also supportive of a suite of mechanisms that contribute to the $\mathrm{CMC}-\mathrm{Cu}$ toxicity seen in S. cerevisiae.

Advancement in nanosynthesis creates a plethora of novel nanomaterials with precisely defined biochemical features. Mammalian cell lines have been found to respond in an equally diverse cellular response to these nanomaterials. The Murine model system and cell lines from Homo sapiens have failed to uptake NP while others uptake NP by a range of endocytosis and resulting in different cellular fates for the $\mathrm{NP}^{11,12}$. In the yeast model, nanoparticles, such as multi-walled carbon nanotubes (MWCNT) and silver (Ag) nanoparticles are endocytosed by S. cerevisiae ${ }^{13,14}$. The antimicrobial activity of any metal nanoparticle is owing from several attributes specific to the particle in question such as the size ${ }^{15}$, shape ${ }^{16}$, surface charge ${ }^{17}$, and their release of metallic ions. Although copper and silver nanoparticles (AgNP) are known to have a strong antimicrobial effect $^{18-20}$, their toxicity varies on their environment. Anaerobically synthesized AgNP cannot subsequentially be oxidized, thus they cannot be ionized and are largely benign to bacteria ${ }^{21}$. The findings of my research implicate that $\mathrm{CMC}-\mathrm{Cu}$ exposure induces regulated cell death $(\mathrm{RCD})$ from evidence found in metal homeostasis and pharmacological intervention by antioxidant rescue of cell death. Additionally, accidental cell death (ACD) is also suspected to play a role in the holistic toxicity of $\mathrm{CMC}-\mathrm{Cu}$, as evident from investigations into the cellular lipids and morphology. Copper, in particular, mediates surface contact killing of microorganisms which may place the cellular lipid peroxidation and composition alteration in context. RCD is inherently dynamic and encompasses a multitude of subroutines and cellular crosstalk between pro-life and pro-death signals that ultimately determines the fate of the cel1 ${ }^{22}$. The field of yeast toxicity has yet to define a point-of-no-return between the reversibility of a stress stimulus and the irrevocable engagement in a lethal cascade ${ }^{22}$. Ionic copper released from $\mathrm{CMC}-\mathrm{Cu}$ contributes to the RCD while localized CuNP present on the cellular surface of yeast contribute to ACD, which follows the general principle in toxicity that not only the form but also the concentration of a given perturbation governs the form of death ${ }^{23,24}$.

It has yet to be determined if these nanometals deter microorganisms from gaining resistance, as seen with soluble copper. But given enough time, with the correct combination of microorganisms and conditions, the possibility of overcoming a once toxic dose of $\mathrm{CMC}-\mathrm{Cu}$ is inherently conceivable. Antibiotic resistance spreads readily among pathogens and the importance of novel antimicrobials is imperative; consequently, studies of nanometal-based antimicrobials will markedly increase during the next decade. In 2013, the global market for nanoparticles in biotechnology and pharmaceuticals touched 25 billion US dollars ${ }^{25}$. The market was expected to have a compound annual growth rate of $22 \%$, growing to over $\$ 80$ billion in $2020^{25}$. The advancement of $\mathrm{CMC}-\mathrm{Cu}$ in insertable devices for the medical biotechnology industry carries potential life-saving technology. The Agency for Healthcare Research and Quality estimates over 35 million people are hospitalized each year in the United States. Of all the hospitalized patients in the world, $7-15 \%$ percent will acquire a healthcare-associated infection ${ }^{26}$ and the rate can be as high as $40 \%$ in Latin America, Sub-Saharan Africa, and Asia ${ }^{27}$. Often associated with these 
infections, are invasive devices employed by modern healthcare professionals ${ }^{28}$. The most common infections are linked with central line-associated bloodstream infections, catheterassociated urinary tract infections, ventilator-associated pneumonia, and surgical-site infections. Catheters placed in the central line to provide fluids and medicine are an example of these insertable devices, which when infected cause a $12-15 \%$ death rate ${ }^{29}$. Normal, plastic catheters serve as a critical reservoir and source for nosocomial pathogens $\mathrm{s}^{30}$. The use of $\mathrm{CMC}-\mathrm{Cu}$ nanomaterials embedded into moldable plastics like polyvinyl alcohol (PVA) can inhibit important medical biofilm bacteria, such as bacillus lincheniformis ${ }^{31}$. Furthermore, as a mucolytic agent, NAC has been shown to reduce the biofilm formation of $E$. coli $^{32}$. To this effect, NAC can be used in a synergistic combination with copper nanoparticles to battle biofilm formation on insertable medical devices without providing rescue to microorganisms. This is an important difference in the toxicological characterization provided by copper nanoparticles that traditional soluble copper lacks.

To identify the additional mechanisms of $\mathrm{CMC}-\mathrm{Cu}$ toxicity, I performed several assays characterizing and quantifying the importance of lipid peroxidation, reactive oxygen species, and genotoxicity. CMC-Cu generates substantial amounts of ROS that likely contribute to the peroxidation of cellular lipids. No evidence of genotoxicity was found and it was not considered to be a substantial factor. By further investigating the cellular metabolism, I found that a lipidomics approach presented the best explanation. I described altered phosphatidylcholine and phosphatidylethanolamine membrane compositions in response to $\mathrm{CMC}-\mathrm{Cu}$ exposure, as well as depleted triacylglycerols, suggesting an impact on the Kennedy lipid pathway which synthesizes the membrane lipids ${ }^{33,34}$. Additionally, I observed distinct cell surface damage via scanning electron micrographs upon $\mathrm{CMC}-\mathrm{Cu}$ exposure that shows similarity to ethanol toxicity and may be indicative of adhesion facilitated rupturing by nanostructures ${ }^{35,36}$.

Taken together, this evidence supported my central hypothesis that the positively charged cellulose copper nanoparticles are attracted to the negative charge of the cell wall where importation leads to ROS damage of the cellular membrane lipids and biomolecules initiating toxicosis of the yeast cell.

In Chapter Four, I furthered my analysis of metabolism beyond the limited lipid metabolism to focus on the metabolomics of a new fermenting yeast species that gained interest from the brewing industry and had not received the GRAS status from the FDA. This chapter explored the metabolism of Saccharomyces species in a broader context using $S$. arboricola. To determine the similarity and differences of the novel species to a GRAS recognized yeast in the same genus, $S$. cerevisiae, I profiled the endogenous and exogenous metabolome of $S$. arboricola, S. cerevisiae, and their hybrids. My rationale for generating hybrids was dual purpose; first to explore my hypothesis that interspecific yeast hybrids between $S$. cerevisiae x $S$. arboricola would gain transgressive phenotypes such as increased ethanol production due to hybrid vigor, which my results support. The second was to simulate the natural hybridization that may occur in the fermentation industry as has been seen in the past between many Saccharomyces species ${ }^{37-41}$.

The volatile metabolites were quantified to yield insight into the sensory profile of their fermentation profiles, impacting the desirable or undesirable flavor aspects of their metabolic differences. The endogenous metabolome analysis failed to reveal any acutely toxic compounds 
produced via fermentation relative to the already GRAS S. cerevisiae. In the ginjo style of Japanese sake, ethanol and ethyl hexanoate are important sensory metabolic compounds suggesting the hybrids possess a high propensity for ginjo sake production. The hybrids had high levels of both ethyl hexanoate, imparting a pleasant fruity aroma, and ethanol. The increased ethanol production of the hybrids and $S$. arboricola is likely owing from the abundance of mannitol in the sake $\operatorname{mash}^{42,43}$. This is suggestive of $S$. arboricola's superior utilization of this sugar alcohol over $S$. cerevisiae $^{44}$. My findings supported my hypothesis that $S$. arboricola and interspecific hybrids are uniquely fit for sake fermentation with A. oryzae because of their metabolic access to the underutilized sugar-alcohol and temperature preference which affects the flavor active metabolites.

Asian $S$. cerevisiae strains used in fermentation are not similar to the $S$. cerevisiae strains used in modern brewing across the world. Written records of brewing history can be aligned with the phylogeny of sequenced $S$. cerevisiae strains when the strains' geographical locations are considered $^{45}$, a tribute to the dispersion pathway and continued domestication of $S$. cerevisiae. Ecological and genetic diversity studies ${ }^{45-51}$ of yeast have similarities to the studies of homo sapiens origin ${ }^{52-57}$. There is great Saccharomyces genetic diversity in Asia. S. arboricola was isolated from Asia, near the theoretical origin of Saccharomyces yeast. This environmental isolation facilitated a genetic divergence between the two species that is reflected in their metabolism and reflective of their resource availability for that duration of time. S. arboricola has the ability to efficiently use mannitol while it also harbors an increased sensitivity to lithium and copper. These traits can be expected because $S$. arboricola lacks amplifications of the lithium exporter ENAI and the copper metallothionein CUPI that determine these traits in $S$. cerevisiae $^{58,59}$. These phenotypes are indicative of the rural niche environment of $S$. arboricola and the contrast between the two Saccharomyces species is indicative of the $S$. cerevisiae's increased resistance to copper when isolated from agricultural environments.

Future research into metabolic capacity and engineering in fermentation is likely to expand on the important ester profiles already present and common in the yeast fermentation profile. Flavoractive esters are critically important in brewing practices due to their fruity qualities and natural concentrations that flirt with human sensory thresholds. Esters are formed intracellularly during fermentation. Acetate (solvent-like), isoamyl acetate (fruity, banana), ethyl caproate and ethyl caprylate (sour apple), and phenyl ethyl acetate (flowery, roses, honey) are monitored both qualitatively and quantitively by consumers and technicians, respectively. Acetate esters diffuse rapidly through the cellular membrane into the fermenting medium because of the solubility in lipids. The fatty acid ethyl ester is released less rapidly into the fermenting medium and diffusion of the aroma active compound is dependent on the tail length: $100 \%$ for ethyl caproate, $60 \%$ for ethyl caprylate, $12 \%$ for ethyl caprate ${ }^{60}$. Currently, there are efforts from several organizations that are aimed at increasing the aroma and organoleptic complexity of fermentations by interspecific hybridizations and genetic manipulation ${ }^{61-65}$.

In summary, this dissertation work has contributed an evidence-based model for $\mathrm{CMC}-\mathrm{Cu}$ nanotoxicity to $S$. cerevisiae. This research adds to the important, second phase of the four progressive levels of understanding nanotoxicity. These levels include the particle characteristics, cellular toxicity, organ toxicity, and in vivo toxicity ${ }^{6}$. Also, this dissertation research has provided 
metabolic insights into $S$. arboricola and interspecific hybrids' use in industrial Asian fermentations that probe at the evolutionary domestication of Saccharomyces strains. 


\section{References}

1. Colomban, P., Tournié, A. \& Ricciardi, P. Raman spectroscopy of copper nanoparticlecontaining glass matrices: Ancient red stained-glass windows. J. Raman Spectrosc. 40, 1949-1955 (2009).

2. Colomban, P. The use of metal nanoparticles to produce yellow, red and iridescent colour, from bronze age to present times in lustre pottery and glass: Solid state chemistry, spectroscopy and nanostructure. J. Nano Res. 8, 109-132 (2009).

3. Underwood, S. \& Mulvaney, P. Effect of the Solution Refractive Index on the Color of Gold Colloids. Langmuir 10, 3427-3430 (1994).

4. Liang, A., Liu, Q., Wen, G. \& Jiang, Z. The surface-plasmon-resonance effect of nanogold/silver and its analytical applications. TrAC - Trends Anal. Chem. 37, 32-47 (2012).

5. Vance, M. E. et al. Nanotechnology in the real world: Redeveloping the nanomaterial consumer products inventory. Beilstein J. Nanotechnol. 6, 1769-1780 (2015).

6. Shin, S., Song, I. \& Um, S. Role of Physicochemical Properties in Nanoparticle Toxicity. Nanomaterials 5, 1351-1365 (2015).

7. Zhong, T. et al. Antimicrobial Properties of the Hybrid Copper NanoparticlesCarboxymethyl Cellulose. Wood Fiber Sci. 45, 215-222 (2013).

8. Rong-Mullins, X. et al. Proteomic and genetic analysis of the response of S. cerevisiae to soluble copper leads to improvement of the antimicrobial function of cellulosic copper nanoparticles. Metallomics 9, 1304-1315 (2017).

9. Clarkson, T. W. Molecular and Ionic Mimicry of Toxic Metals. Annu. Rev. Pharmacol. Toxicol. 545-571 (1993).

10. Bridges, C. C. \& Zalups, R. K. Ionic and molecular mimicry and the transport of metals. Cell. Mol. Biol. Met. 204, 241-294 (2010).

11. Oh, N. \& Park, J. H. Endocytosis and exocytosis of nanoparticles in mammalian cells. Int. J. Nanomedicine 9, 51-63 (2014).

12. Behzadi, S. et al. Cellular Uptake of Nanoparticles: Journey Inside the Cell. Chem. Soc. Rev. 46, 4218-4244 (2017).

13. Käosaar, S., Kahru, A., Mantecca, P. \& Kasemets, K. Profiling of the toxicity mechanisms of coated and uncoated silver nanoparticles to yeast Saccharomyces cerevisiae BY4741 using a set of its 9 single-gene deletion mutants defective in oxidative stress response, cell wall or membrane integrity and endocyt. Toxicol. Vitr. 35, 149-162 (2016).

14. Phillips, C. L., Yah, C. S., Iyuke, S. E. \& Rumbold, K. The cellular response of Saccharomyces cerevisiae to multi-walled carbon nanotubes ( MWCNTs ). J. Saudi Chem. Soc. 19, 147-154 (2015).

15. Morones, J. R. et al. The bactericidal effect of silver nanoparticles. Nanotechnology 16, 2346-2353 (2005).

16. Pal, S., Tak, Y. K. \& Song, J. M. Does the antibacterial activity of silver nanoparticles depend on the shape of the nanoparticle? A study of the gram-negative bacterium Escherichia coli. Appl. Environ. Microbiol. 73, 1712-1720 (2007).

17. Badawy, A. M. E. L., Silva, R. G., Morris, B., Scheckel, K. G. \& Suidan, M. T. Surface Charge-Dependent Toxicity of Silver Nanoparticles. Environ. Sci. Technol. 283-287 (2011).

18. Sondi, I. \& Salopek-Sondi, B. Silver nanoparticles as antimicrobial agent: A case study on E. coli as a model for Gram-negative bacteria. J. Colloid Interface Sci. 275, 177-182 (2004).

19. Stoimenov, P. K., Klinger, R. L., Marchin, G. L. \& Klabunde, K. J. Metal oxide 
nanoparticles as bactericidal agents. Langmuir 18, 6679-6686 (2002).

20. Ruparelia, J. P., Chatterjee, A. K., Duttagupta, S. P. \& Mukherji, S. Strain specificity in antimicrobial activity of silver and copper nanoparticles. Acta Biomater. 4, 707-716 (2008).

21. Xiu, Z. M., Zhang, Q. B., Puppala, H. L., Colvin, V. L. \& Alvarez, P. J. J. Negligible particle-specific antibacterial activity of silver nanoparticles. Nano Lett. 12, 4271-4275 (2012).

22. Carmona-Gutierrez, D. et al. Guidelines and recommendations on yeast cell death nomenclature. Microb. cell (Graz, Austria) 5, 4-31 (2018).

23. Shen, H. M. \& Vandenabeele, P. Necrotic cell death. Necrotic Cell Death (2014). doi:10.1007/978-1-4614-8220-8

24. Denton, D., Nicolson, S. \& Kumar, S. Cell death by autophagy: Facts and apparent artefacts. Cell Death Differ. 19, 87-95 (2012).

25. Jackson Highsmith. Nanoparticles in Biotechnology, Drug Development and Drug Delivery: BIO113B | BCC Research. Global Markets (2014). Available at: https://www.bccresearch.com/market-research/biotechnology/nanoparticles-

biotechnology-drug-development-drug-delivery-report.html. (Accessed: 30th January 2020)

26. Danasekaran, R., Mani, G. \& Annadurai, K. Prevention of healthcare-associated infections: protecting patients, saving lives. Int. J. Community Med. Public Heal. 1, 67 (2014).

27. Allegranzi, B. et al. Burden of endemic health-care-associated infection in developing countries: Systematic review and meta-analysis. Lancet 377, 228-241 (2011).

28. Centers for disease control and prevention. Types of Healthcare-associated Infections. 2014 (2014). Available at: https://www.cdc.gov/HAI/infectionTypes.html. (Accessed: 30th January 2020)

29. Srinivasan, A. et al. Vital signs: Central line-associated blood stream infections - United States, 2001, 2008, and 2009. Morb. Mortal. Wkly. Rep. 60, 243-248 (2011).

30. Warren, J. W. Catheter-associated urinary tract infections. Int. J. Antimicrob. Agents 17, 299-303 (2001).

31. Winans, M. J., Gallagher, J. E. G., Jaczynski, J. \& Oporto, G. Pick Your Poison: Benzalkonium Chloride and Copper Enable Nanocellulose Derivatives to Form Antimicrobial Properties Against a Spectrum of Microorganisms. bioRxiv 783076 (2019). doi:10.1101/783076

32. Marchese, A. et al. Effect of fosfomycin alone and in combination with N-acetylcysteine on E. coli biofilms. Int. J. Antimicrob. Agents 22, 95-100 (2003).

33. Farine, L., Niemann, M., Schneider, A. \& Bütikofer, P. Phosphatidylethanolamine and phosphatidylcholine biosynthesis by the Kennedy pathway occurs at different sites in Trypanosoma brucei. Sci. Rep. 5, 1-11 (2015).

34. Gibellini, F. \& Smith, T. K. The Kennedy pathway-de novo synthesis of phosphatidylethanolamine and phosphatidylcholine. IUBMB Life 62, 414-428 (2010).

35. Luo, Y. et al. Effect of yeast cell morphology, cell wall physical structure and chemical composition on patulin adsorption. PLoS One 10, (2015).

36. Nowlin, K., Boseman, A., Covell, A. \& LaJeunesse, D. Adhesion-dependent rupturing of Saccharomyces cerevisiae on biological antimicrobial nanostructured surfaces. J. R. Soc. Interface 12, (2014).

37. Chambers, P. J. et al. Ongoing domestication of wine yeast: Past, present and future. Aust. J. Grape Wine Res. (2015). doi:10.1111/ajgw.12190 
38. Gibson, B. \& Liti, G. Saccharomyces pastorianus : genomic insights inspiring innovation for industry. 17-27 (2015). doi:10.1002/yea

39. Smart, K. a. Brewing yeast genomes and genome-wide expression and proteome profiling during fermentation. Yeast 24, 993-1013 (2007).

40. Naumov, G. I., Masneuf, I., Naumova, E. S., Aigle, M. \& Dubourdieu, D. Association of Saccharomyces bayanus var. uvarum with some French wines: Genetic analysis of yeast populations. Res. Microbiol. 151, 683-691 (2000).

41. Wendland, J. Lager Yeast Comes of Age. Eukaryot. Cell 13, 1256-1265 (2014).

42. Song, S. H. \& Vieille, C. Recent advances in the biological production of mannitol. Appl. Microbiol. Biotechnol. 84, 55-62 (2009).

43. Smiley, K. L., Cadmus, M. C. \& Liepins, P. Biosynthesis of D-mannitol from D-glucose by Aspergillus candidus. Biotechnol. Bioeng. 9, 365-374 (1967).

44. Liti, G. et al. High quality de novo sequencing and assembly of the Saccharomyces arboricolus genome. BMC Genomics 14, 14:69 (2013).

45. Gallone, B. et al. Domestication and Divergence of Saccharomyces cerevisiae Beer Yeasts. Cell 166, 1397-1410.e16 (2016).

46. Duan, S. F. et al. The origin and adaptive evolution of domesticated populations of yeast from Far East Asia. Nat. Commun. 9, (2018).

47. Nespolo, R. F. et al. An Out-of-Patagonia dispersal explains most of the worldwide genetic distribution in Saccharomyces eubayanus. bioRxiv 709253 (2019). doi:10.1101/709253

48. Peter, J. et al. Genome evolution across 1,011 Saccharomyces cerevisiae isolates. Nature 556, 339-344 (2018).

49. Wang, Q. M., Liu, W. Q., Liti, G., Wang, S. A. \& Bai, F. Y. Surprisingly diverged populations of Saccharomyces cerevisiae in natural environments remote from human activity. Mol. Ecol. 21, 5404-5417 (2012).

50. Bing, J., Han, P. J., Liu, W. Q., Wang, Q. M. \& Bai, F. Y. Evidence for a far east asian origin of lager beer yeast. Curr. Biol. 24, R380-R381 (2014).

51. Boynton, P. J. \& Greig, D. The ecology and evolution of non-domesticated Saccharomyces species Primrose. Yeast 31, 449-462 (2014).

52. Henn, B. M. et al. Hunter-gatherer genomic diversity suggests a southern African origin for modern humans. Proc. Natl. Acad. Sci. U. S. A. 108, 5154-5162 (2011).

53. Henn, B. M. et al. Distance from sub-Saharan Africa predicts mutational load in diverse human genomes. Proc. Natl. Acad. Sci. U. S. A. 113, E440-E449 (2016).

54. Relethford, J. H. Genetic evidence and the modern human origins debate. Heredity (Edinb). 100, 555-563 (2008).

55. Yu, N. et al. Larger genetic differences within Africans than between Africans and Eurasians. Genetics 161, 269-274 (2002).

56. Choudhury, A., Aron, S., Sengupta, D., Hazelhurst, S. \& Ramsay, M. African genetic diversity provides novel insights into evolutionary history and local adaptations. Hum. Mol. Genet. 27, R209-R218 (2018).

57. Campbell, M. C. \& Tishkoff, S. A. AFRICAN GENETIC DIVERSITY: Implications for Human Demographic History, Modern Human Origins, and Complex Disease Mapping. Annu. Rev. Genomics Hum. Genet. 9, 403-433 (2008).

58. Warringer, J. et al. Trait variation in yeast is defined by population history. PLoS Genet. 7, (2011).

59. Liti, G. et al. High quality de novo sequencing and assembly of the Saccharomyces 
arboricolus genome. BMC Genomics 14, 14:69 (2013).

60. Verstrepen, K. J. et al. Flavor-active esters: Adding fruitiness to beer. J. Biosci. Bioeng. 96, 110-118 (2003).

61. Bellon, J. R., Ford, C. M., Borneman, A. R. \& Chambers, P. J. A novel approach to isolating improved industrial interspecific wine yeasts using chromosomal mutations as potential markers for increased fitness. Front. Microbiol. 9, 1-12 (2018).

62. Bellon, J. R., Schmid, F., Capone, D. L., Dunn, B. L. \& Chambers, P. J. Introducing a New Breed of Wine Yeast: Interspecific Hybridisation between a Commercial Saccharomyces cerevisiae Wine Yeast and Saccharomyces mikatae. PLoS One 8, (2013).

63. Bellon, J. R. et al. Newly generated interspecific wine yeast hybrids introduce flavour and aroma diversity to wines. Appl. Microbiol. Biotechnol. 91, 603-612 (2011).

64. Mertens, S. et al. A large set of newly created interspecific Saccharomyces hybrids increases aromatic diversity in lager beers. Appl. Environ. Microbiol. 81, 8202-8214 (2015).

65. Steensels, J. et al. Selecting and generating superior yeasts for the brewing industry. Cerevisia 37, 63-67 (2012). 
END 
